\title{
OPINION
}

\section{Pitfalls of artificial grouping and stratification of scientific journals based on their Impact Factor: a case study in Brazilian Zoology}

\begin{abstract}
The present contribution explores the impact of the QUALIS metric system for academic evaluation implemented by CAPES (Coordination for the Development of Personnel in Higher Education) upon Brazilian Zoological research. The QUALIS system is based on the grouping and ranking of scientific journals according to their Impact Factor (IF). We examined two main points implied by this system, namely: 1) its reliability as a guideline for authors; 2) if Zoology possesses the same publication profile as Botany and Oceanography, three fields of knowledge grouped by CAPES under the subarea "BOZ" for purposes of evaluation. Additionally, we tested CAPES' recent suggestion that the area of Ecology would represent a fourth field of research compatible with the former three. Our results indicate that this system of classification is inappropriate as a guideline for publication improvement, with approximately one third of the journals changing their strata between years. We also demonstrate that the citation profile of Zoology is distinct from those of Botany and Oceanography. Finally, we show that Ecology shows an IF that is significantly different from those of Botany, Oceanography, and Zoology, and that grouping these fields together would be particularly detrimental to Zoology. We conclude that the use of only one parameter of analysis for the stratification of journals, i.e., the Impact Factor calculated for a comparatively small number of journals, fails to evaluate with accuracy the pattern of publication present in Zoology, Botany, and Oceanography. While such simplified procedure might appeals to our sense of objectivity, it dismisses any real attempt to evaluate with clarity the merit embedded in at least three very distinct aspects of scientific practice, namely: productivity, quality, and specificity.
\end{abstract}

KEY WORDS. CAPES; IF; JIF; QUALIS; scientometry.

The quantification of academic merit is not an easy task. Although it plays a central role in the academic environment, it is largely undefined in objective terms (Lewis \& Doyno 1983) and there are increasing doubts that it can be approached in a simple or operational way (Bollen et al. 2009). In spite of these problems, in the past few years the scientific community witnessed an increasing concern from governmental agencies around the world to determine metrical systems capable of evaluating, for funding purposes, the productivity of scientists, institutions and graduate programs through the impact of published research.

One of the first initiatives in Brazil intended to formalize a metric system for academic evaluation took place in 1996 and 1997, when the Ministry of Education, represented by the Coordination for the Development of Personnel in Higher Education (CAPES), established a format that sought to evaluate the Brazilian Graduate Programs in all available fields of knowledge (SouzA \& PAULA 2002). For that purpose, CAPES developed the QUALIS database, which provides rankings of journals and books in each of the 47 main areas of knowledge defined by CAPES (see: http:/ /qualis.capes.gov.br/webqualis/EmailRepresentantesAreas.faces).
Each main area is regulated by a specific committee, which is in charge of defining rules for ranking all journals and books related to the area in question. Journals related to natural sciences, such as Zoology, Botany, and Ecology, have been traditionally ranked only through their impact factor (IF), as calculated by the Institute of Scientific Information (ISI/Web of Knowledge). The IF is defined as the ratio between the citations that a journal receives in a period of two years by the number of articles published by that journal in the same period, and was originally proposed as a way to objectively measure and compare the relevance of journals to the scientific community (GARFIELD 2006). Thus CAPES evaluates the quality of each Graduate Program through the IF of the journals in which the articles of the researchers associated to that specific Program are published. As explicitly stated by CAPES (see additional Information in Appendix 1": "Reestruturação do QUALIS 2009"), one of the main intents of the QUALIS database is to redirect the Brazilian scientific production towards journals with higher IFs, ultimately discouraging Brazilian scientists to publish in journals with low IFs.

Although the system has its own merits, it fails to take into account the specificities of many areas of research, forc-

\footnotetext{
${ }^{1}$ Available as Online Supplementary Material accessed with the online version of the manuscript at http://www.scielo.br/zool
} 
ing an unwarranted competition between distinct areas of knowledge, and even between different disciplines within the same field of knowledge. Strong criticisms have been recently raised against the QUALIS system in such distinct areas as Medicine (e.g. Rocha-e-Silva 2009), Chemistry (e.g. Andrade \& Galembeck 2009), and Zoology (Penteado Filho 2009, Langguth 2009). Although we found common grounds in most criticisms raised within these areas, we will focus our analysis in Zoology as a case study that can be extended to other fields.

Brazil is the most biodiverse country in the world, encompassing approximately $13 \%$ (c.a. 1.8 million species) of the global biodiversity (Lewinsohn \& Prado 2005). Such unique natural resources stimulated the establishment of a strong tradition in Zoological research (ZARUR 1994), with a large and dynamic academic community, several long-standing societies, three major zoological museums, and an unprecedented contribution in the exploration and study of South American fauna (Michán \& Lloentes-Bousquet 2010). This tradition rests heavily on an anatomical and descriptive biology, with a major part of the Brazilian zoologists working in the field of systematics and taxonomy (MARQues \& LAMAS 2006).

The use of the IF in the evaluation of taxonomy has long been thought as extremely problematic and incapable of expressing true academic merit for this area (Krell 2000, 2001, MinelLI 2003). It is probably for that reason that the release of the 2009 QUALIS report generated a strong negative reaction from the zoological community. Criticisms toward the new QUALIS culminated in an open letter from the Brazilian Society of Zoology, signed by 130 zoologists, botanists, editors, and Graduate Program coordinators, questioning the methods of evaluation of the scientific production implemented by the agency and, more specifically, the use of the journals' IF as the only criterion of evaluation (a copy of the open letter can be found at http:// www.sbzoologia.org.br/noticia_interna.php?idnoticia $=75$; see also Penteado Filho 2009).

Even though the validity of the IF have been strongly questioned as an indexing criterion (e.g. SEGLEN 1997, GLÄNZEL \& Moed 2002, Garfield 2006, Ewing 2006, Adler et al. 2008, and references therein), the real effects of its use as the only criterion for academic merit in Brazilian Zoology are still not clear. In the present contribution, we explore the use of the IF by CAPES, and its impacts in the evaluation of Brazilian Zoological research. Two main points implied by the QUALIS classification were evaluated, namely: if the QUALIS database can be reliable as a normative factor to guide researchers towards more appropriate choices of journals for publishing; if Botany, Oceanography, and Zoology possess the same publication profiles and can thus be grouped together in a cohesive area of research, as defined by QUALIS. Additionally, we tested CAPES' recent suggestion that the area of Ecology would represent a fourth field of research with a similar publication profile as the one determined by Botany, Oceanography, and Zoology.

\section{MATERIAL AND METHODS}

\section{QUALIS database}

The QUALIS database consists of a list of indexed journals belonging to a specific area of knowledge that are ranked according to a given criterion defined by a committee of specialists. The list of criteria used may be significantly distinct between the areas of knowledge. An area of knowledge might correspond to a field of research, such as Biochemistry, or may include several distinct fields of research such as Zoology and Genetics. In the context of the QUALIS database, Zoology finds itself within the area called "Biological Sciences I" ("Ciências Biológicas I"; called here "CBI"), which includes also the fields of Genetics, General Biology, Botany, and Oceanography.

Recently, the CBI committee decided to subdivide the area in two subareas, "GBG" and "BOZ", with the former encompassing the fields of Genetics and "General Biology" and the latter including Botany, Oceanography and Zoology. Such subdivision was justified by the committee as a necessary step to remove discrepancies due to a "bimodal heterogeneity" present in the IFs of the two subareas. The new subdivision was implemented for the evaluation triennium of 2007-2009, and allowed the CBI committee to calculate median scores and strata thresholds for BOZ and GBG separately. Table I and figure 1 represent, respectively, a section of the list of journals used by the CBI committee to calculate the median of BOZ, and the threshold values of the six strata depicted as a scale for the stratification of $\mathrm{BOZ}$ journals.

The subarea BOZ is composed by 719 scientific journals. From these, 441 were indexed in JCR and had IF calculated for at least the last 10 years. Journals directly related to the field of Zoology were identified based on the scope of the journal and the topic of the papers published. We also included several "multidisciplinary" journals that were non-taxon-specific, but were of main interest for zoologists in general (e.g. American Naturalist [ISSN: 0003-0147], Cladistics [ISSN: 0748-3007], Systematic Biology [ISSN: 1063-5157]). The list resulted in a total of 274 journals related to the field of Zoology, from which 147 presented indexed IF.

\section{Data acquisition and statistical analyses}

We obtained a list of journals from the CBI area of the QUALIS database for the year of 2009 in the WEBQUALIS webpage (http://qualis.capes.gov.br/webqualis/). CBI journals were subsequently organized in separate GBG and BOZ groups, based on the documentation provided by the CBI committee (see Appendix 22: "Lista de revistas e cálculo do IF mediano da BOZ"). The present analysis will focus on the BOZ group.

\footnotetext{
${ }^{2}$ Available as Online Supplementary Material accessed with the online version of the manuscript at http://www.scielo.br/zool
} 
Table I. Excerpt from the table used by the QUALIS committee to calculate the median of the group of journals included in BOZ. Different tones of gray are used to highlight clusters of articles from the same journal.

\begin{tabular}{|c|c|c|c|}
\hline Journal & Paper information & ISSN & IF \\
\hline Science & v. 1, first p. 303 , last p. 357 & 0036-8075 & 30.028 \\
\hline Science & v. 307 , first p. 1044 , last p. 1047 & 0036-8075 & 30.028 \\
\hline Science & v. 311 , first p. 73 , last p. 77 & 0036-8075 & 30.028 \\
\hline Science & v. 312, first p. 1917 , last p. 1934 & 0036-8075 & 30.028 \\
\hline Science & v. 313 , first p. 48 , last p. 50 & 0036-8075 & 30.028 \\
\hline Science & v. 314 , first p. 1541 , last p. 1543 & 0036-8075 & 30.028 \\
\hline Nature (London) & v. 440 , first p. 1037 , last p. 1040 & $0028-0836$ & 26.681 \\
\hline Nature (London) & v. 443 , first p. 931 , last p. 949 & 0028-0836 & 26.681 \\
\hline Plant Cell & v. 16, first p. 1314 , last p. 1326 & $1040-4651$ & 9.868 \\
\hline Proceedings of the National Academy & v. 102 , first p. 18502 , last p. 18507 & $0027-8424$ & 9.643 \\
\hline Systematic Biology (Philadelphia) & v. 53 , first p. 767 , last p. 780 & $1063-5157$ & 7.748 \\
\hline Systematic Biology (Philadelphia) & v. 55 , first p. 97 , last p. 115 & $1063-5157$ & 7.748 \\
\hline PLOS Genetics & v. 2 , first p. 1012 , last p. 1024 & $1553-7390$ & 7.671 \\
\hline Plant Journal & v. 44 , first p. 707 , last p. 717 & $0960-7412$ & 6.565 \\
\hline Journal of Cell Science & v. 119, first p. 2486 , last p. 2496 & $0021-9533$ & 6.427 \\
\hline Plant Physiology (Bethesda) & v. 134 , first p. 951 , last p. 959 & $0032-0889$ & 6.125 \\
\hline Plant Physiology (Bethesda) & v. 135, first p. 287 , last p. 299 & $0032-0889$ & 6.125 \\
\hline Revista Brasileira de Zoologia & v. 23, first p. 841 , last p. 864 & 0101-8175 & 0 \\
\hline Revista Brasileira de Zoologia & v. 23 , first p. 865 , last p. 869 & $0101-8175$ & 0 \\
\hline Revista Brasileira de Zoologia & v. 23 , first p. 877 , last p. 879 & 0101-8175 & 0 \\
\hline Revista Brasileira de Zoologia & v. 23, first p. 879 , last p. 882 & 0101-8175 & 0 \\
\hline Revista Brasileira de Zoologia & v. 23, first p. 886, last p. 887 & 0101-8175 & 0 \\
\hline Revista Brasileira de Zoologia & v. 23 , first p. 888 , last p. 890 & 0101-8175 & 0 \\
\hline Revista Brasileira de Zoologia & v. 23 , first p. 891 , last p. 893 & 0101-8175 & 0 \\
\hline Revista Brasileira de Zoologia & v. 23 , first p. 901 , last p. 914 & 0101-8175 & 0 \\
\hline Revista Brasileira de Zoologia & v. 24 , first p. 847 , last p. 855 & 0101-8175 & 0 \\
\hline Revista Brasileira de Zoologia & v. 32 , first p. 350 , last p. 356 & 0101-8175 & 0 \\
\hline Revista Brasileira de Zoologia & v. 32 , first p. 460 , last p. 470 & 0101-8175 & 0 \\
\hline Revista Brasileira de Zoologia & v. 35 , first p. 483 , last p. 488 & 0101-8175 & 0 \\
\hline Revista Brasileira de Zoologia & v. 6, first p. 115, last p. 127 & 0101-8175 & 0 \\
\hline Revista Brasileira de Zoologia & v. n. 22 , first p. 613 , last p. 618 & $0101-8175$ & 0 \\
\hline Median & & & 0.612 \\
\hline
\end{tabular}


According to CAPES, the QUALIS database is reliable as a normative factor. In order to evaluate this assertion, we computed the IFs of all journals classified as $\mathrm{BOZ}$ for the years of 2006 and 2008, using the strata defined by QUALIS for the triennium of 2007-2009, and quantified the number of journals that changed their category between 2006 and 2008. This was calculated for Zoology alone and for the whole group of journals pertaining to BOZ (Tab. II).

According to the committee, the subdivision of CBI in the subareas of BOZ and GBG was stimulated by the argument that both subareas have distinct publication profiles. To test this hypothesis, we computed the difference between the medians shown by BOZ and Zoology. We then carried out a resampling procedure (MANLY 1997) intended to identify if such claimed differences are significant: 10,000 random samples of $\mathrm{N}$ journals were drawn from $\mathrm{BOZ}$ without replacement, with $\mathrm{N}$ being the number of journals that were assigned to the Zoology subarea. We then estimated the median of the IF for each sample and calculated the difference between the obtained value and the observed median of BOZ. This procedure resulted in an empirical distribution of the differences of medians between $\mathrm{BOZ}$ and the subsamples with size N. The empirical Pvalue was then calculated as the proportion of permutations that produced absolute differences superior to that found between the observed values. In other words, if Zoology were actually a subarea of BOZ, we would expect the observed differences between medians to fall within the range of differences estimated from random samples of $\mathrm{BOZ}$ with the same size. Finally, we repeated this procedure for the years of 1998 to 2004,2006 , and 2008, with the intent of evaluating the historical trend of these areas. The years of 2005 and 2007 were not available for the present study.

The recent suggestion made by the CBI committee that the area of Ecology would represent a fourth field of research with a similar publication profile exhibited by Botany, Oceanography, and Zoology was tested with the same resampling procedure described above. We randomly sampled $\mathrm{N}$ journals from a "Biodiversity" group composed of journals from Ecology (drawn from the Ecology QUALIS area), Botany, Oceanography and Zoology, with $\mathrm{N}$ being the number of BOZ journals. This procedure can be understood as a test to investigate if Ecology and $\mathrm{BOZ}$ do show a similar citation pattern. The same test was extended for all available years.

\section{RESULTS AND DISCUSSION}

\section{The QUALIS stratification}

The CBI committee performed a stratification of the journals based on the scientific production of all officially recognized graduate programs associated to the fields of Zoology, Botany, and Oceanography. The procedure consisted in the elaboration of a list of all papers published in the last three years by researchers belonging to the graduate programs of Zoology, Botany, and Oceanography. CAPES retrieved this information from the annual reports sent every year by each graduate program, and in which a detailed list of the scientific production is provided. The resulting lists of publications are summed together, removing repeated entries of the same article. Each paper receives a score equivalent to the IF of the journal in which the article was published. Journals that lack a calculated IF in ISI were not taken into account, except for a rooster of 10 indicated journals, chosen by the committee and added to the QUALIS calculation with an IF equal to zero (Tab. I). To establish a basis for the stratification, the committee calculated the median of these scores and the thresholds of the strata were then defined over this value, multiplied by different factors for each threshold (Fig. 1). As a result of this procedure, journals belonging to very distinct fields of knowledge were included in the list of journals used for the calculation of the median for the CBI area (Fig. 2).

This procedure could be regarded, at first glance, to represent a fair methodology of evaluation. However, it does not

Table II. Total numbers and percentages of changes computed for journals pertaining to the BOZ group along the QUALIS strata, between the years of 2006 (base year of the QUALIS evaluation) and 2008. (N) Number of journals listed for each specific stratum; (Change) number of journals that changed its stratum between 2006 and 2008; $(<)$ number of journals that changed to a lower stratum between 2006 and 2008; (>) number of journals that changed to a higher stratum between 2006 and 2008.

\begin{tabular}{|c|c|c|c|c|c|c|c|c|}
\hline \multirow{2}{*}{ QUALIS strata } & \multicolumn{4}{|c|}{$\mathrm{BOZ}$} & \multicolumn{4}{|c|}{ Zoology } \\
\hline & $\mathrm{N}$ & Change & $<$ & $>$ & $\mathrm{N}$ & Change & $<$ & $>$ \\
\hline $\mathrm{A} 1$ & 119 & $23(19 \%)$ & $23(19 \%)$ & - & 23 & $6(26 \%)$ & $6(26 \%)$ & - \\
\hline $\mathrm{A} 2$ & 101 & $30(30 \%)$ & $18(18 \%)$ & $12(12 \%)$ & 24 & $10(42 \%)$ & $9(38 \%)$ & $1(0.5 \%)$ \\
\hline B1 & 136 & $40(29 \%)$ & $15(11 \%)$ & $25(18 \%)$ & 57 & $14(24 \%)$ & $6(11 \%)$ & $8(14 \%)$ \\
\hline B2 & 47 & $31(66 \%)$ & $14(30 \%)$ & $17(36 \%)$ & 23 & $15(65 \%)$ & $12(52 \%)$ & $3(13 \%)$ \\
\hline B3 & 35 & $19(54 \%)$ & $6(17 \%)$ & $13(37 \%)$ & 19 & $10(53 \%)$ & $3(16 \%)$ & $7(37 \%)$ \\
\hline B4 & 2 & - & - & - & 1 & - & - & - \\
\hline Total & 440 & $143(33 \%)$ & $76(17 \%)$ & $67(15 \%)$ & 147 & $55(37 \%)$ & $36(24 \%)$ & $19(13 \%)$ \\
\hline
\end{tabular}

ZOOLOGIA 27 (4): 493-502, August, 2010 


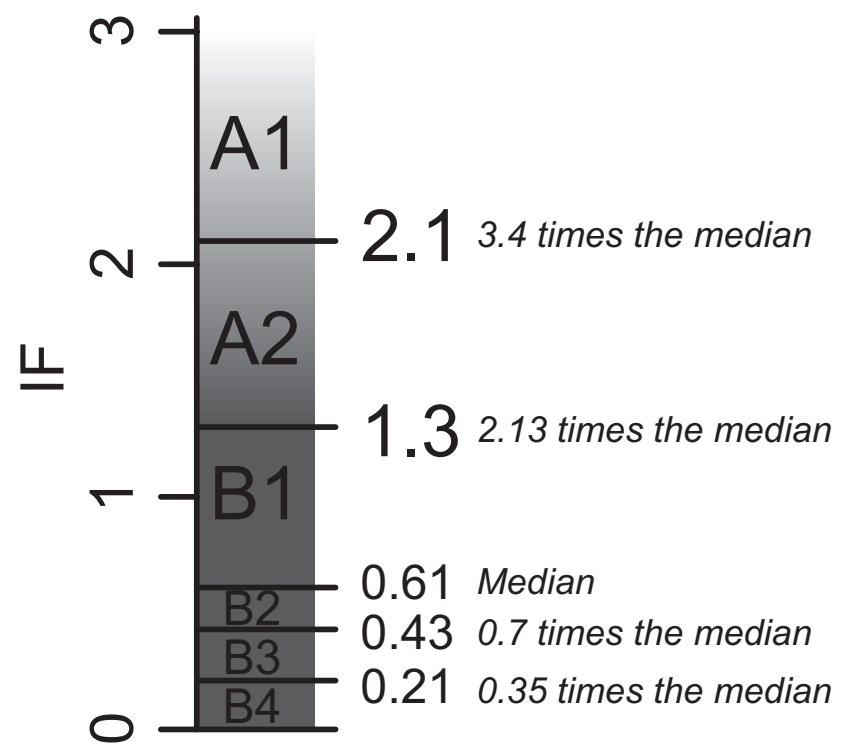

Figure 1. QUALIS stratification of the $\mathrm{BOZ}$ group, showing the values of the stratum thresholds for the triennium 2007-2009. appear to be the case for at least the BOZ subarea evaluated in the present study. Publication listings obtained from the graduate programs belonging to $\mathrm{BOZ}$ were not filtered in order to retain only the journals related to the fields of Zoology, Botany, and Oceanography. Indeed, all journals were used in the calculations, including those from fields of knowledge that are only distantly or not at all related to BOZ (e.g., Engineering, Medicine; Fig. 2), and which IFs are incompatible with the latter (present study; AMIN \& Mabe 2000). However, the implications of such a heterogeneous list of journals composing the subarea $\mathrm{BOZ}$ is still poorly understood. That said, the procedure proposed by CAPES seems to be unnecessarily convoluted: it only serves to produce an arbitrary parameter of stratification, a goal that could be achieved through the use of any value of multiplication based on the median.

\section{Stability of the strata in the QUALIS system}

In order to serve as a normative factor, the strata proposed in QUALIS should be stable along the years. In other words, if one intends to publish in the upper strata of QUALIS (i.e., A1 and A2), journals in these given strata would preferably stay within the same stratum from one triennium to the other.

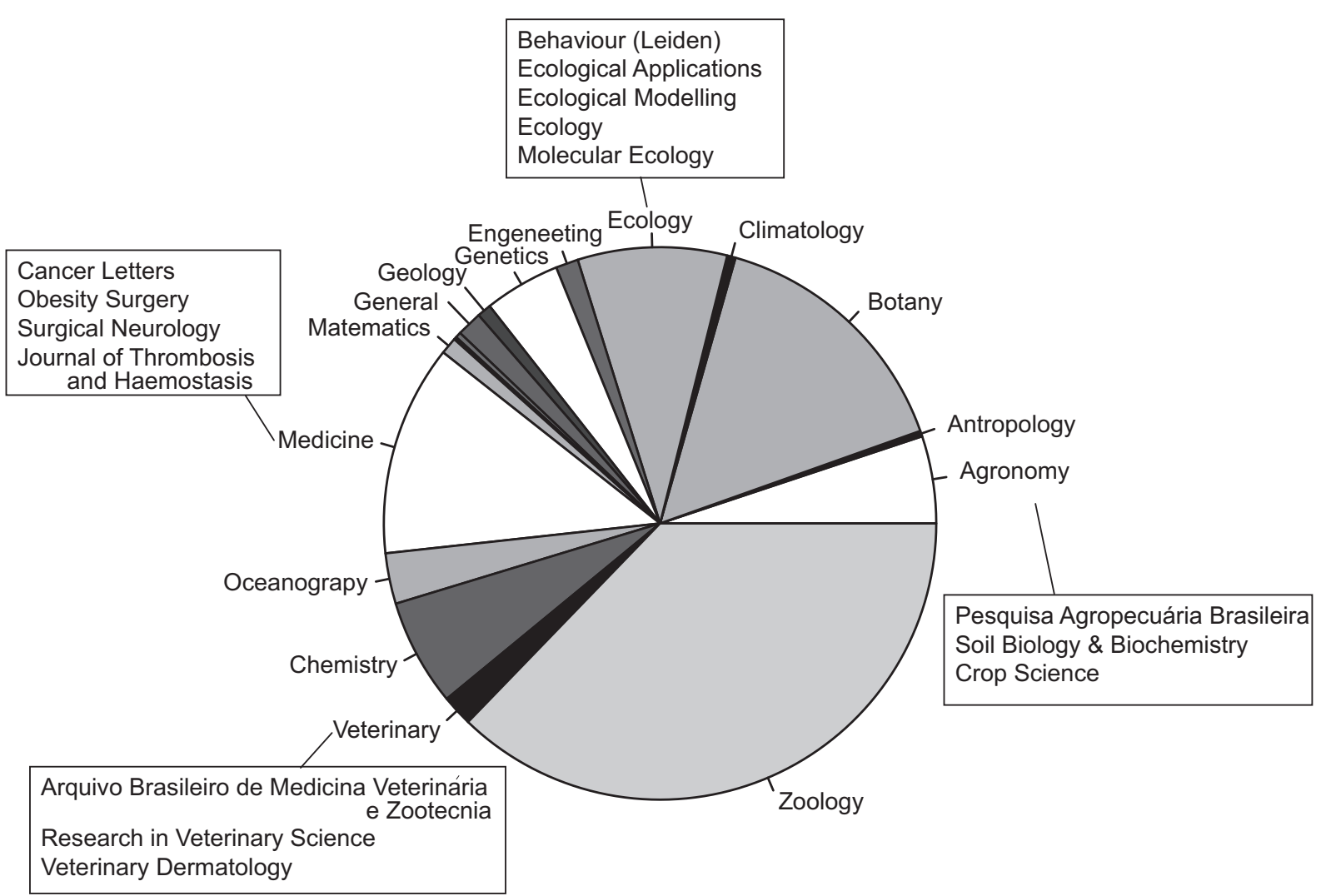

Figure 2. Relative proportion of the journals used to calculate the median for the BOZ group, and separated by areas of knowledge. Boxes provide examples of the journals that belong to these specific areas of knowledge and were used in the calculation of the median for BOZ. 
Journals from BOZ and Zoology, however, presented highly unstable IF values between the years of 2006 and 2008 (Figs 3-6), with approximately one third of the 440 journals changing from a stratum to another (Tab. II). The majority of the journals that changed their status had a decrease in their IF, thus being classified in a lower stratum. A significant number of journals belonging to stratum A1 showed a decrease in IF, with 19 and $26 \%$ of the journals being classified in lower strata for $\mathrm{BOZ}$ and Zoology, respectively. Strata B2 and B3 were particularly un- stable, with more than half of their journals being classified in other strata, resulting in an almost complete overlap between them. B4 surprisingly presented no misclassifications due to the fact that most journals classified in this stratum lack an IF score for 2006 and 2008, indicating that this stratum is mainly comprised of newly indexed journals.

Our analysis shows that the QUALIS method of stratification proposed by the committee is inappropriate as a guideline for publication improvement, with approximately one
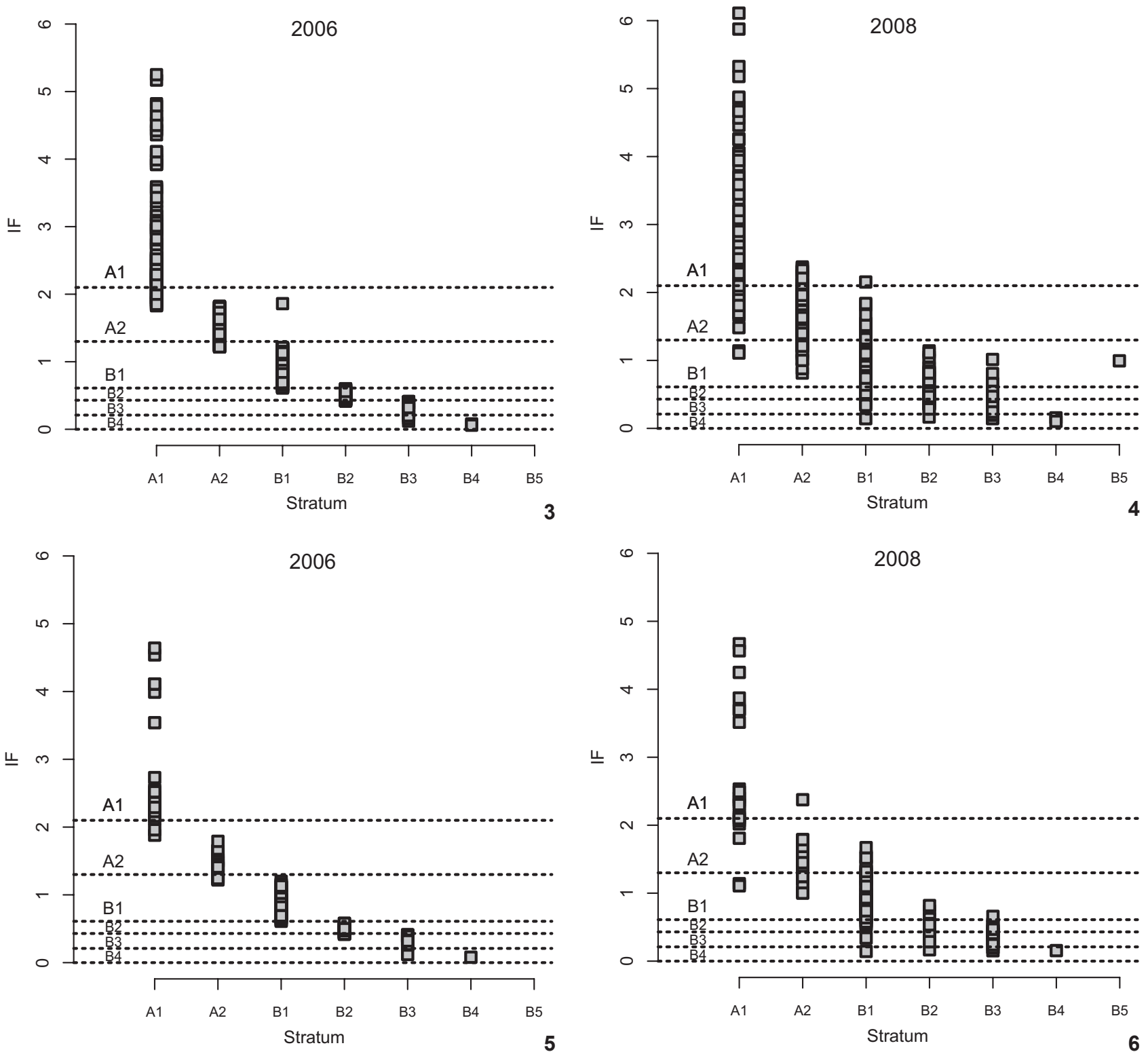

Figures 3-6. Impact Factor scores, separated by strata, of journals from BOZ (4 and 5) and Zoology (6 and 7) of the QUALIS database of the years 2006 and 2008. Dashed lines represent the six strata thresholds (A1 to B5). 
third of the journals (for both BOZ and Zoology) changing their strata between years. This result is not surprising: even though the IF can be considered generally stable along the years (GLänZEI $\&$ Moed 2002), stochastic factors can have a great deal of influence in changes between the years (Amin \& Mabe 2000, Ogden $\&$ BARTLEY 2008). These factors are more relevant for journals that publish fewer articles due to the increased influence of individual articles (ADLER et al. 2008). This pattern can be particularly damaging when coupled with the instability of QUALIS stratification and its normative aspects. Publications with lower IF and fewer articles published would be more subject to larger proportional random fluctuations in their IF. If the IF is the sole basis for QUALIS, then these journals would likely be dismissed as unreliable, a process that could happen within $\mathrm{BOZ}$ and Zoology (Tab. II), with journals from these fields being effectively classified two ranks away from their original stratum (Figs 3-6). The small absolute differences between the strata thresholds (Fig. 1) probably contribute to this problem, rendering the QUALIS strata inadequate to guide zoologists in their decisions.

\section{The BOZ grouping}

Historically, Zoology is much more stable than BOZ, presenting a relatively constant median IF between 1998 and 2004, with a moderate increase from 2004 to 2008. BOZ, however, shows a bimodal pattern of increase, with a moderate slope between 1998 and 2003 and a more angled one from 2003 to 2008 (Fig. 7). The permutation analysis indicates that the median IF for Zoology cannot be found through random samples within BOZ journals for all years, except for the year of 1998 (Tab. III). It is clear that from 2002 to 2008 , Zoology completely diverged from the expected trajectory of median IFs for BOZ (Fig. 7).

This divergence seems to impact negatively the classification of Zoology journals (Fig. 8). While Botany and Oceanography show almost half of their journals in the two uppermost strata, Zoology journals tend to be classified in lower strata, with only one third of them in the upper ones (A1 and A2). It is worth noticing that journals from BOZ that were clearly related to the area of Ecology, such as Chemoecology [ISSN: 0937-7409], Conservation Biology [ISSN: 0888-8892] and Journal of Tropical Ecology [ISSN: 0266-4674], presented an evident bias towards the upper strata. This is expected since ecological journals do tend to show higher IFs (Fig. 7) and are thus "better" classified when grouped with journals that tend to show lower IFs.

CBI subareas were intended to deal with the obvious incompatibilities between distinct fields with conspicuously disparate IF values (see QUALIS documentation in http:// qualis.capes.gov.br/webqualis/). Although the segregation between BOZ and GBG correctly addressed the main dichotomy found in previous versions of QUALIS, it does not address the individual characteristics of Botany, Oceanography and Zool-

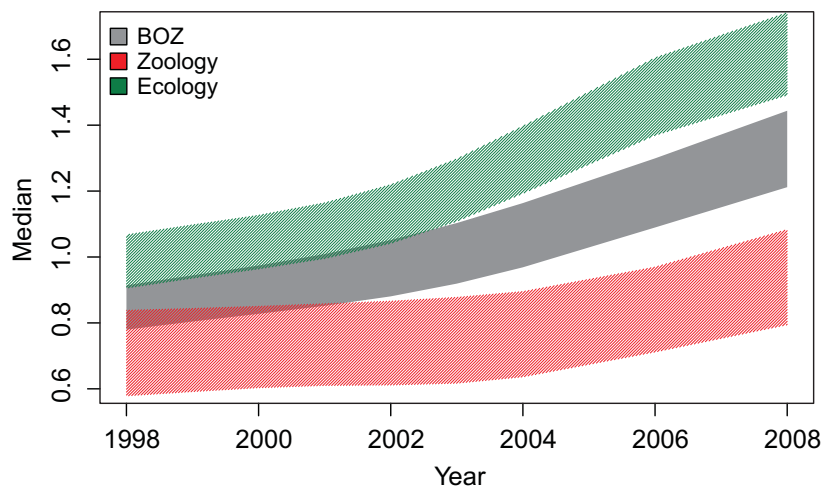

Figure 7. Evolution of the impact factor (IF) median along a 10 year period for BOZ area (gray), Zoology subarea (red) and Ecology area (green), showing the $95 \%$ confidence interval estimated through bootstrapping (10,000 re-samplings). All estimates were smoothed using a spline function for graphical purposes.

Table III. Median of the IF for each analyzed year with empirical P-values of the difference of medians based on the resampling procedure for each year (see text for details). Numbers in bold represent P-values smaller than $\alpha=0.05$.

\begin{tabular}{|c|c|c|c|c|c|}
\hline \multirow{2}{*}{ Years } & \multicolumn{3}{|c|}{ Median } & \multicolumn{2}{|c|}{ P-value } \\
\hline & Zoology & $\mathrm{BOZ}$ & Biodiversity & BOZ-Zoology & Biodiversity-BOZ \\
\hline 1998 & 0.742 & 0.841 & 0.955 & 0.05515 & 0.59896 \\
\hline 1999 & 0.730 & 0.900 & 1.020 & 0.00182 & 0.04694 \\
\hline 2000 & 0.707 & 0.895 & 1.033 & 0.00037 & 0.00520 \\
\hline 2001 & 0.802 & 0.919 & 1.057 & 0.03888 & 0.70867 \\
\hline 2002 & 0.705 & 0.937 & 1.084 & 0.00148 & 0.00387 \\
\hline 2003 & 0.698 & 0.967 & 1.152 & $4.00 \mathrm{E}-05$ & 0.00049 \\
\hline 2004 & 0.704 & 1.060 & 1.227 & $>1.00 \mathrm{E}-05$ & $>1.00 \mathrm{E}-05$ \\
\hline 2006 & 0.860 & 1.215 & 1.470 & $>1.00 \mathrm{E}-05$ & $6.00 \mathrm{E}-05$ \\
\hline 2008 & 0.927 & 1.333 & 1.500 & $>1.00 \mathrm{E}-05$ & $>1.00 \mathrm{E}-05$ \\
\hline
\end{tabular}


ogy. Our analysis shows that the insertion of Zoology within $\mathrm{BOZ}$ is artificial, and that it cannot be considered a valid subsample of this subarea of CBI. This grouping is actually detrimental for Zoology, as it tends to present lower IFs, reinforcing the tendency shown by Zoology journals to score poorly under the QUALIS stratification scheme (Fig. 8).
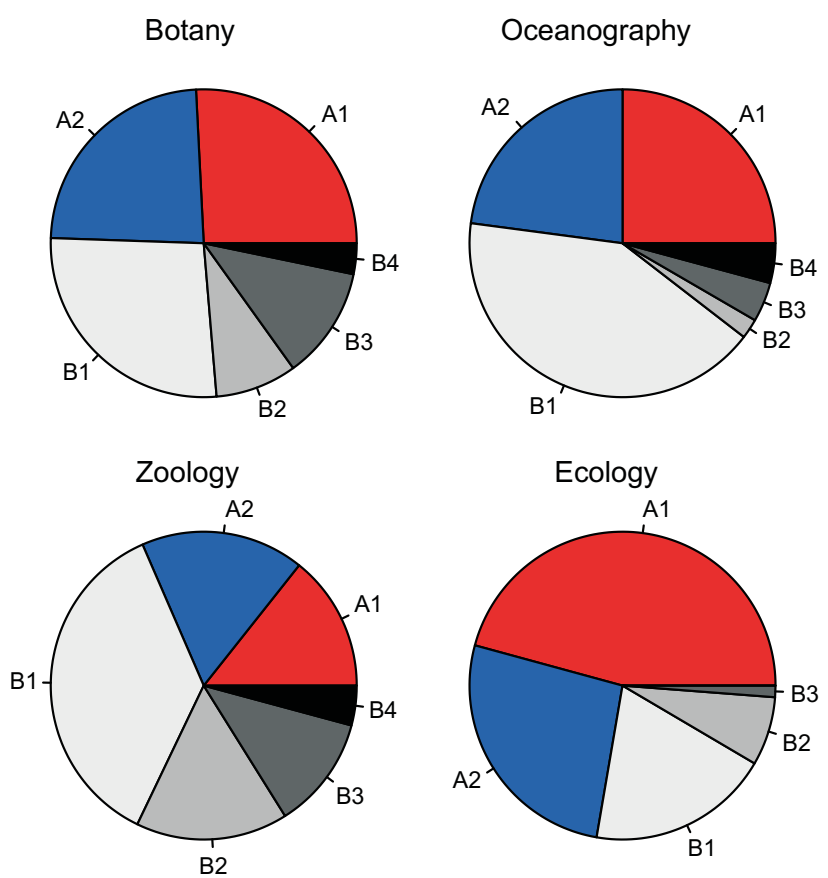

Figure 8. Relative proportion of journals from the fields of Botany, Oceanography, Zoology and Ecology that belong to the BOZ subarea of QUALIS, classified in the six distinct QUALIS strata (A1 to B4) defined by $\mathrm{BOZ}$ (see figure 1).

It could be argued that this is a legitimate trend for the field of Zoology, and that the lower IFs and, consequently, lower QUALIS classification actually reflects a poor academic performance. It is known, however, that comparisons between distinct areas are not straightforward: each discipline (and even subdisciplines) has its own tradition and pattern of citations (EwING 2006), affecting the meaning and validity of IF for these different areas of knowledge. In Zoology, and more specifically in taxonomy, the meaning of the IF is patently decoupled from the principle of academic merit (Krell 2000, 2001, Minelui 2003). Taxonomic papers do not tend to be cited within the "window" of two years of IF calculations, rendering this measure of impact almost meaningless for this field (KReLl 2000, 2001,
Minelur 2003). Under these conclusions, the clear bias shown by the sole use of IF values for evaluation of scientific journals reinforces the impression that the QUALIS system might threaten in the near future the growing effort by zoologists to describe the Brazilian biodiversity (MARques \& LAMAS 2006, Michán \& Llorentes-BousQuet 2010).

\section{The "Biodiversity" group}

A recent presentation given by CAPES officials highlighted their intention, for the next triennium (2010-2012), to merge BOZ journals with journals from the field of Ecology, forming a new QUALIS area called "Biodiversity". This new grouping was justified through a theoretical perspective, instead of a purely scientometric one. Indeed, according to the document from CAPES, the areas involved show "thematic affinities and interfaces" that justify a "Biodiversity area" 3 . However, although the involved fields of knowledge likely demonstrate a common theoretical background, it is still arguable that they share a common scientometric profile.

Journals belonging to the Ecology area show a moderate initial increase of their IF from 1998 to 2003, with a more angled slope appearing from 2003 to 2006 that decreases slightly from 2006 to 2008 (Fig. 7). The area of Ecology in QUALIS not only shows a more accelerated increase of its historical IF, but also presents a higher initial IF than that of Zoology (Fig. 7).

Our preliminary evaluation of the Biodiversity area reveals that $\mathrm{BOZ}$ could not be interpreted as a sub-sample of a greater area that combines both Ecology and $\mathrm{BOZ}$ journals. The differences were significant for all years, except for 1998 and 2001 (Tab. III). Despite these exceptions, the pattern is very clear and shows that the creation of a Biodiversity area would underestimate all fields represented in BOZ.

Our results indicate that the creation of a Biodiversity area would result in a highly heterogeneous field in terms of citation profiles, a problem that was supposedly addressed through the subdivision of CBI. The grouping of Ecology with $\mathrm{BOZ}$ would lead to an unfair competition between different areas. The higher IF of Ecology would guarantee a higher classification in QUALIS for the journals of this area, downplaying the importance of basic science, such as Zoology and Botany. The drawbacks of this practice could be two-fold: 1) mainly descriptive sciences, such as Zoology, would be forced to compete in unreasonable terms, and 2) mainly experimental sciences, such as Ecology, would see a significant number of journals migrate to higher strata of the Qualis system without any real improvement of their IF.

\section{Final remarks}

The Brazilian scientific community reacted to the newest version of the QUALIS with perplexity. Many editorials were quick to point out that the new methodology did not reflect academic merit and was downplaying the role of classical jour-

\footnotetext{
${ }^{3}$ Diretrizes da CBI-CAPES para os Programas de Pós-Graduação, Brasília, August, 2009.
} 
nals and probably of whole areas of research (RocHA-E-SILVA 2009, Andrade \& Galembeck 2009). The most unified response came from the societies of Zoology and Botany in the form of an open letter expressing deep concern for the use of the IF as the only index of evaluation of academic merit (see also Penteado FilHo 2009, LANGGUTH 2009). We agree with these criticisms and have presented here a comprehensive evaluation of the potential pitfalls of the procedure adopted by the CBI committee for the BOZ group and, more specifically, for the field of Zoology.

The meaning of the IF in distinct fields of scientific knowledge has been discussed at length, and the validity of its use as an absolute measure of merit have been barely supported by researchers in scientometry (e.g. Glänzel \& MoEd 2002, EwING 2006, AdLer et al. 2008). Nevertheless, the growing culture in favor of "objectivity" has lead administrators and policymakers to embrace the IF as a quick tool for decision-making, ignoring concomitantly the basic question of what is being actually measured (EWING 2006).

The new QUALIS definitely joined this global trend, ignoring any thoughtful evaluation of the meaning of "merit." The reinforcement of the IF as the only parameter used by governmental agencies, such as CAPES and CNPq, to evaluate scientific merit could have more profound consequences on Brazilian research cycles. A continuous pressure to publish high impact articles might indeed alter scientific practices by stimulating data falsification (FANELL 2010a) and selective publishing of positive results (FAnell 2010b). Furthermore, the focus on IF could lean unjustified pressure on national journals that do not conform to the required pattern of citation, possibly leading to less governmental funding and incentive. More troublesome, however, are the already visible collateral effects on descriptive sciences, such as taxonomy, as opposed to experimental sciences. While Zoology and Botany definitely fall in the first group, Ecology tends to be included in the second one. This dichotomy seems to be illustrated in our analysis by a discrepant gap between these fields (Fig. 7). The decision to unite them in a single "Biodiversity" area will increase the already existing gap between Zoology and other fields included in BOZ. Brazilian Zoology is a very competitive field when evaluated independently (ADAMs \& King 2010, Michán \& LloRentes-Bousquet 2010), and its insertion in artificial groups could intensify the already troubling global trend of marginalization of Organismal Biology, undermining the long-term achievements of the Brazilian "National Zoology Program" (CARVALHo et al. 2007).

One could argue that the only way to evaluate the merit of researchers from a specific field of knowledge would be to analyze the quality of their publication (EwING 2006). Although we do agree with this argument, we believe that the new QUALIS method of stratification does not represent an appropriate solution for this endeavor. The procedure used for the stratification of the journals reduces all aspects related to the pattern of publications present in Zoology, Botany, and Oceanography to only one parameter of analysis, i.e., the impact factor calcu- lated for a comparatively small number of journals. While such simplification might appeal to our sense of objectivity, it dismisses any real attempt to evaluate with clarity at least three very distinct aspects of scientific practice that seem to represent "academic merit," namely: productivity, quality, and specificity. For QUALIS to become an efficient and uncontroverted evaluation tool of academic merit, it must incorporate more than one criterion of evaluation (not only the IF of journals), in order to take into account the specificity of the area being analyzed, for a better and fair judgment of the productivity of the scientist and quality of his research.

Any attempt of evaluation of academic merit should rests upon procedures that clearly take into account these three factors. A clear delimitation of the specificity of the area pool of journals or researchers to be evaluated is crucial for the outcome of a fair result.

\section{ACKNOWLEDGEMENTS}

We are deeply indebted to Abel Packer, Rogerio Meneghini, Rogerio Mugnaini, Walter A. Boeger, Marcelo Carvalho, Luis F. Silveira, Miguel T. Rodrigues, Celio Haddad, Augusto Abe, Carlos A. N. Iannini, Ana Lúcia da C. Prudente, Rodney R. Cavichioli, Sionei R. Bonatto, Luciane Marinoni, Carlos Lamas, Marcos Tavares, Carlos R. F. Brandão, Mario de Vivo, Mario de Pinna, Alfredo Langguth for helpful and enlightening discussions about scientific production and impact factors. We are also grateful to Pedro Gnaspini and Marcio de C. Silva Filho for providing the BOZ list of journals used by CBI to calculate the QUALIS stratification for the subarea. This research was supported by the Fundação de Amparo à Pesquisa do Estado de São Paulo BIOTA/ FAPESP, Grant number 02/13602-4 (to H.Z.).

\section{LITERATURE CITED}

Adams, J. \& C. King. 2010. Global Research Report: Brazil. Research and collaboration in the new geography of science. UK, Thomson Reuters, Evidence Ltd. Available online at: http://researchanalytics.thomsonreuters.com/m/ pdfs/GRR-Brazil-Jun09.pdf [Accessed: 9.VI.2010].

Adler, R.; J. Ewing \& P. Taylor. 2008. Citation Statistics, a Report from the Joint Committee on Quantitative Assessment of Research (IMU, ICIAM, IMS). Available online at: http:/ /www.ams.org/ewing/Documents/CitationStatistics-FINAL1.pdf [Accessed: 9.VI.2010].

Amin M. \& M. Maвe. 2000. Impact factors: use and abuse. Perspectives in Publishing. Oxford, Elsevier Science, Newsletter no. 1. Available online at: http://www.igh.org/ publicaccess/Perspectives1.pdf [Accessed: 11.VI.2010].

Andrade J. B. \& F. Galembeck. 2009. QUALIS: Quo Vadis?. Química Nova 32: 5. doi: 10.1590/S0100-40422009000100001.

Bollen J.; H.V. Sompel; A. Hagberg \& R. Chute. 2009. A principal component analysis of 39 scientific impact measures. PLoS ONE 4 (6): e6022. doi:10.1371/journal.pone.0006022. 
Carvalho, M.R.; F.A. Bockmann; D.S. Amorim; C.R.F. Brandão; M. Vivo; J.L. Figueiredo; H.A. Britski; M.C.C. Pinna; N.A. Menezes \& F.P.L. MARQUES. 2007. Taxonomic impediment or impediment to taxonomy? A commentary on systematics and the cybertaxonomic-automation paradigm. Evolutionary Biology 34 (3): 140-143. doi: 10.1007/s11692-007-9011-6.

EwING, J. 2006. Measuring journals. Notices of the American Mathematical Society 53 (9): 1049-1053. Available online at: www.ams.org/notices/200609/comm-ewing.pdf [Accessed: 9.VI.2010].

Fanelli, D. 2010a. How many scientists fabricate and falsify research? A systematic review and meta-analysis of survey data. PLoS ONE 4 (5): e5738. doi:10.1371/journal.pone.0005738.

FANELLI, D. 2010b. Do pressures to publish increase scientists' bias? An empirical support from US States data. PLoS ONE 5 (4): e10271. doi:10.1371/journal.pone.0010271.

Garfield, E. 2006. The history and meaning of the Journal Impact Factor. Journal of the American Medical Association 295 (1): 90-93.

Glänzel, W. \& H.F. Moed. 2002. Journal impact measures in bibliometric research. Scientometrics 53 (2): 171-193. doi: 10.1023/A:1014848323806.

KRELL, F.T. 2000. Impact factors aren't relevant to taxonomy. Nature 405: 507-508. doi:10.1038/35014664.

KRELL, F.T. 2001. Why impact factors don't work for taxonomy. Nature 415: 957. doi:10.1038/415957a.

LANGguth, A. 2009. Ditadura da CAPES. Boletim da Sociedade Brasileira de Mastozoologia 54: 11-12.

Lewinsohn, T.M. \& P.I. Prado. 2005. How many species are there in Brazil? Conservation Biology 19 (3): 619-624. doi:10.1111/j.1523-1739.2005.00680.x.

Lewis, L.S. \& V. Doyno. 1983. The definition of academic merit. Higher Education, 12: 707-719. doi: 10.1007/BF00132426.
MAnly, B.F.J. 1997. Randomization, Bootstrap and Monte Carlo Methods in Biology. London, Chapman and Hall, 461p.

Marques, A.C. \& C.J.E. Lamas. 2006. Taxonomia zoológica no Brasil: estado da arte, expectativas e sugestões de ações futuras. Papéis Avulsos de Zoologia 46: 139-174. doi: 10.1590/S0031-10492006001300001.

Michán L. \& J. Llorente-Bousquets. 2010. Bibliometria de la sistematica biologica sobre America Latina durante el siglo XX en tres bases de datos mundiales. Revista de Biología Tropical/International Journal of Tropical Biology and Conservation 58 (2): 531-554.

Minelli, A. 2003. The status of taxonomic literature. Trends in Ecology \& Evolution 18 (2): 75-76. doi:10.1016/S01695347(02)00051-4.

Ogden, T.L. \& D.L. Bartley. 2008. The ups and downs of journal impact factors. Annals of Occupational Hygiene 52 (2): 73-82. doi:10.1093/annhyg/men002.

Penteado Filho, R.C. 2009. O problema não é o mérito dos periódicos. Boletim da Sociedade Brasileira de Mastozoologia 54: 10-11.

RochA-E-SILVA, M. 2009. O novo Qualis, que não tem nada a ver com a ciência do Brasil. Carta aberta ao presidente da CAPES. Clinics 64 (8): 721-724. doi: 10.1590/S180759322009000800002.

Seglen, P.O. 1997. Citations and journal impact factors questionable indicators of research quality. Allergy 11: 10501056.

Souza, E.P. \& M.C.S. PAula. 2002. QUALIS: a base de qualificação dos periódicos científicos utilizada na avaliação CAPES. INFOCAPES - Boletim Informativo da CAPES 2002 abriljunho 10 (2): 7-25.

ZARUR, G.C.L. 1994. Schools and paradigms in Brazilian Zoology. Interciencia 19 (4): 183-190.

\section{Fábio A. Machado' \& Hussam Zaher ${ }^{1,2}$}

\footnotetext{
${ }^{1}$ Museu de Zoologia, Universidade de São Paulo. Avenida Nazaré 481, Ipiranga, 04263-000 São Paulo, SP, Brazil.

2 Corresponding author. E-mail: hzaher@usp.br
}

Submitted: 28.VI.2010; Accepted: 29.VII.2010.

Editorial responsibility: Walter A.P. Boeger 
Appendix 1. Document regarding the restructuring of the QUALIS system for the year 2009.

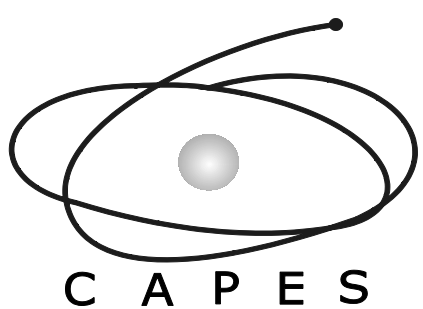

Ministério da Educação - MEC

Coordenação de Aperfeiçoamento de Pessoal de Nível Superior - CAPES Diretoria de Avaliação - DAV

\section{REESTRUTURAÇÃO DO QUALIS}

O Conselho Técnico Científico da Educação Superior, em reunião realizada nos dias 16 e 17 de abril, para a qual foram convidados todos os coordenadores de área, dos quais 43 se fizeram presentes ou foram representados por seus adjuntos (37 titulares e 06 adjuntos), considerando os resultados dos debates efetuados com base na contribuição inicial recebida do anterior CTC e as conclusões a que chegaram os três grupos de trabalho, integrados por coordenadores de áreas em exercício, delibera o seguinte sobre o processo de reestruturação do Qualis da Capes:

1. O Qualis continua sendo o referencial da produção intelectual apresentada pelos programas de pós-graduação e avaliada pela Capes.

2. Para assegurar a maior transparência entre as áreas e junto à comunidade, a classificação de Periódicos de cada área deve ser aprovada pelo CTC-ES.

3. Cada área deve definir o que considera "periódico", e apenas os veículos de divulgação que atendam o estabelecido nesse conceito serão classificados no respectivo Qualis de Periódicos. Em princípio, somente os veículos com corpo editorial reconhecido, com avaliação pelos pares (pareceristas ad hoc) e dotados de ISSN devem ser considerados periódicos. Além disso, no caso das áreas que apresentam critérios de indexação bem estabelecidos, suas bases de dados podem, para esse fim, ser utilizadas como referência.

4. O Qualis será composto de oito estratos, a saber:

— A1, o mais elevado;

- A2;

- B1;

- B2;

- B3; 
-B4;

- B5;

- C - com peso zero.

5. Recomenda-se, para se evidenciar o caráter classificatório e discriminante do Qualis de Periódicos, que as áreas povoem, significativamente, pelo menos cinco dos estratos acima, com número significativo de periódicos - não sendo considerado, nesse cômputo, o oitavo estrato, " $\mathrm{C}$ ".

6. Recomenda-se que haja uma diferença significativa entre os pesos atribuídos aos estratos utilizados, de forma que seja evidenciada uma efetiva diferenciação entre eles.

7. Cada área deverá apresentar em seu documento "Critérios do Qualis de Periódicos", os critérios pelos quais define cada estrato (fator de impacto, índice $\mathrm{H}$, ou outros modos de mensurar sua qualidade). Destaca-se a necessidade de a definição dos dois estratos superiores ser efetuada de maneira particularmente criteriosa e rigorosa.

8. Recomenda-se que os dois estratos superiores não sejam superpovoados, a fim de que seja devidamente destacada a excelência ou o diferencial de qualidade dos periódicos neles classificados, em relação aos incluídos nos demais estratos.

9. Os dois estratos superiores - A1 e A2 - poderão ser deixados vazios, por decisão da área, em termos de artigos efetivamente publicados, recomendando-se que deles constem apenas periódicos da mais alta qualidade, nos quais a área julga que deva sinalizar para avançar a qualidade da produção científica de sua comunidade. O Qualis assim terá um papel indutor claramente definido, não se limitando a analisar onde a área publica, mas indicando também onde se deve publicar;

10. Recomenda-se, nas grandes áreas em que for possível e, em especial, naquelas em que já é tradição, como as Engenharias e a Saúde, que se mantenha a política de um Qualis único ou de regras únicas para o Qualis.

11. Recomenda-se que, pelo menos no interior de cada Grande Área, se procure formular regras de Qualis próximas entre si, mas sem, de forma alguma, gerar depreciação das áreas que têm maior tradição de publicação em periódicos ou sobrevalorização daquelas que ainda têm pouco volume nesse campo; portanto, um periódico pode obter notas diferentes em distintas áreas. 
Appendix 2. Table used by the QUALIS committee to calculate the median of the group of journals included in BOZ. Each line corresponds to an article publish by researchers associated to post-graduate programs related to Botany, Oceanography and Botany. This table was provided by the members of the QUALIS committee.

\begin{tabular}{|c|c|c|c|c|}
\hline Journal & Paper info. & ISSN & IF & Half-Life \\
\hline Science & v. 1, fisrt page 303 , last page 357 & $0036-8075$ & 30.028 & 7.7 \\
\hline Science & v. 307, fisrt page 1044, last page 1047 & 0036-8075 & 30.028 & 7.7 \\
\hline Science & v. 311 , fisrt page 73 , last page 77 & 0036-8075 & 30.028 & 7.7 \\
\hline Science & v. 312, fisrt page 1917, last page 1934 & $0036-8075$ & 30.028 & 7.7 \\
\hline Science & v. 313 , fisrt page 48 , last page 50 & 0036-8075 & 30.028 & 7.7 \\
\hline Science & v. 314, fisrt page 1541, last page 1543 & 0036-8075 & 30.028 & 7.7 \\
\hline Nature (London) & v. 440 , fisrt page 1037, last page 1040 & 0028-0836 & 26.681 & 7.8 \\
\hline Nature (London) & v. 443, fisrt page 931, last page 949 & $0028-0836$ & 26.681 & 7.8 \\
\hline Plant Cell & v. 16 , fisrt page 1314 , last page 1326 & $1040-4651$ & 9.868 & 5.6 \\
\hline $\begin{array}{l}\text { Proceedings of the National Academy of } \\
\text { Sciences of the United States of America }\end{array}$ & v. 102 , fist page 18502, last page 18507 & 0027-8424 & 9.643 & 7.1 \\
\hline Systematic Biology (Philadelphia) & v. 53 , fistt page 767 , last page 780 & $1063-5157$ & 7.748 & \\
\hline Systematic Biology (Philadelphia) & v. 55, fisrt page 97, last page 115 & $1063-5157$ & 7.748 & \\
\hline PLOS Genetics & v. 2 , fisrt page 1012 , last page 1024 & $1553-7390$ & 7.671 & 1.2 \\
\hline Plant Journal & v. 44 , fisrt page 707, last page 717 & 0960-7412 & 6.565 & 5.5 \\
\hline Journal of Cell Science & v. 119 , fisrt page 2486, last page 2496 & $0021-9533$ & 6.427 & 5.2 \\
\hline Plant Physiology (Bethesda) & v. 134, fisrt page 951, last page 959 & 0032-0889 & 6.125 & 7.2 \\
\hline Plant Physiology (Bethesda) & v. 135, fisrt page 287, last page 299 & 0032-0889 & 6.125 & 7.2 \\
\hline Neuropsychopharmacology (New York) & v. 30, fisrt page 1246, last page 1256 & 0893-133X & 5.889 & 4.4 \\
\hline Journal of Biological Chemistry & v. 281 , fisrt page 1 , last page 3 & 0021-9258 & 5.808 & 6.6 \\
\hline Neurobiology of Aging & v. 26 , fisrt page 69 , last page 76 & $0197-4580$ & 5.599 & 5.6 \\
\hline Bioscience (Washington) & v. 55, fisrt page 207, last page 217 & 0006-3568 & 5.424 & 8 \\
\hline Journal of Thrombosis and Haemostasis & v. 4 , fistt page 1107 , last page 1113 & $1538-7933$ & 5.138 & 2.3 \\
\hline Molecular Ecology & v. 14, fistt page 2389 , last page 2402 & $0962-1083$ & 4.825 & 5.1 \\
\hline Molecular Ecology & v. 15 , fisrt page 1 , last page 3 & $0962-1083$ & 4.825 & 5.1 \\
\hline Molecular Ecology & v. 15, fisrt page 2305, last page 2315 & $0962-1083$ & 4.825 & 5.1 \\
\hline Molecular Ecology & v. 15, fisrt page 3969, last page 3982 & $0962-1083$ & 4.825 & 5.1 \\
\hline Molecular Ecology & v. 15, fisrt page 4487, last page 4497 & 0962-1083 & 4.825 & 5.1 \\
\hline Ecology & v. 14 , fisrt page 1334 , last page 1343 & $0012-9658$ & 4.782 & 10 \\
\hline Ecology & v. 87, fisrt page 803 , last page 808 & $0012-9658$ & 4.782 & 10 \\
\hline American Naturalist & v. 167 , fisrt page 177, last page 189 & $0003-0147$ & 4.66 & 10 \\
\hline $\begin{array}{l}\text { Current Issues in Molecular Biology } \\
\text { (Online) }\end{array}$ & v. 6 , fisrt page 57 , last page 72 & $1467-3045$ & 4.481 & 2.9 \\
\hline Global Change Biology & v. 10, fisrt page 1, last page 14 & $1354-1013$ & 4.339 & 4.1 \\
\hline Global Change Biology & v. 10 , fisrt page 563 , last page 591 & $1354-1013$ & 4.339 & 4.1 \\
\hline Global Change Biology & v. 10, fisrt page 683, last page 692 & $1354-1013$ & 4.339 & 4.1 \\
\hline Evolution (Lancaster) & v. 58, fisrt page 1303, last page 1317 & 0014-3820 & 4.292 & 10 \\
\hline Journal of Ecology & v. 92, fisrt page 929 , last page 944 & $0022-0477$ & 4.239 & 10 \\
\hline Journal of Ecology & v. 93, fisrt page 863, last page 878 & $0022-0477$ & 4.239 & 10 \\
\hline $\begin{array}{l}\text { Biochimica et Biophysica Acta. } \\
\text { Bioenergetics }\end{array}$ & v. 1757 , fisrt page 1520 , last page 1528 & $0005-2728$ & 4.237 & 6.1 \\
\hline Plant, Cell and Environment & v. 29 , fisrt page 2153 , last page 2167 & 0140-7791 & 4.135 & 7.3 \\
\hline Quaternary Science Reviews & v. 25 , fisrt page 1110 , last page 1126 & $0277-3791$ & 4.113 & 5.4 \\
\hline Mutation Research & v. 605 , fisrt page 87 , last page 93 & $0027-5107$ & 4.111 & 5.1 \\
\hline Environmental Science \& Technology & v. 40 , fisrt page 7642 , last page 7648 & 0013-936X & 4.04 & 6.3 \\
\hline BMC Genomics & v. 5 , fisrt page 84 , last page 110 & $1471-2164$ & 4.029 & 2.1 \\
\hline Infection and Immunity & v. 72 , fisrt page 508 , last page 514 & $0019-9567$ & 4.004 & 7 \\
\hline Journal of Bacteriology & v. 187 , fisrt page 5568, last page 5577 & $0021-9193$ & 3.993 & 8.7 \\
\hline Journal of Bacteriology & v. 186 , fisrt page 2164 , last page 2172 & $0021-9193$ & 3.993 & 8.7 \\
\hline Applied Catalysis. B, Environmental & v. 59, fisrt page 197, last page 203 & 0926-3373 & 3.942 & 4.7 \\
\hline Molecular Plant-Microbe Interactions & v. 17 , fisrt page 827 , last page 836 & 0894-0282 & 3.936 & 6 \\
\hline Journal of Organic Chemistry & v. 86 , fisrt page 957, last page 961 & 0022-3263 & 3.79 & 9.4 \\
\hline Hormones and Behavior & v. 50 , fisrt page 484 , last page 488 & $0018-506 x$ & 3.789 & 5.9 \\
\hline Conservation Biology & v. 19 , fisrt page 595 , last page 600 & 0888-8892 & 3.762 & 7.2 \\
\hline Conservation Biology & v. 19 , fisrt page 625 , last page 631 & 0888-8892 & 3.762 & 7.2 \\
\hline Conservation Biology & v. 19, fisrt page 632, last page 639 & 0888-8892 & 3.762 & 7.2 \\
\hline Conservation Biology & v. 19 , fisrt page 659 , last page 664 & 0888-8892 & 3.762 & 7.2 \\
\hline Conservation Biology & v. 19 , fisrt page 689 , last page 694 & 0888-8892 & 3.762 & 7.2 \\
\hline
\end{tabular}




\begin{tabular}{|c|c|c|c|c|}
\hline Journal & Paper info. & ISSN & $\mathrm{IF}$ & Half-Life \\
\hline Conservation Biology & v. 19 , fisrt page 695 , last page 700 & 0888-8892 & 3.762 & 7.2 \\
\hline Conservation Biology & v. 19 , fisrt page 701 , last page 706 & 0888-8892 & 3.762 & 7.2 \\
\hline Conservation Biology & v. 19 , fisrt page 734 , last page 739 & 0888-8892 & 3.762 & 7.2 \\
\hline Differentiation (London) & v. 72, fisrt page 198, last page 208 & $0301-4681$ & 3.745 & 5.9 \\
\hline Differentiation (London) & v. $74-10$, fisrt page 562 , last page 572 & $0301-4681$ & 3.745 & 5.9 \\
\hline Obesity Surgery & v. 15, fistt page 788, last page 793 & $0960-8923$ & 3.723 & 3.2 \\
\hline $\begin{array}{l}\text { American Journal of Physiology. } \\
\text { Regulatory, Integrative and Comparative }\end{array}$ & v. 289 , fist page 1280 , last page 1288 & 0363-6119 & & \\
\hline Physiology & & & 3.685 & 6.7 \\
\hline Journal of Experimental Botany & v. 56 , fisrt page 1797 , last page 1804 & $0022-0957$ & 3.63 & 5.8 \\
\hline Journal of Experimental Botany & v. 56, fisrt page 1965, last page 1974 & $0022-0957$ & 3.63 & 5.8 \\
\hline Journal of Experimental Botany & v. 56, fisrt page 865 , last page 877 & 0022-0957 & 3.63 & 5.8 \\
\hline Journal of Experimental Botany & v. 57 , fisrt page 3243 , last page 3258 & 0022-0957 & 3.63 & 5.8 \\
\hline Journal of Experimental Botany (Online) & v. 57 , fisrt page 559 , last page 569 & $1460-2431$ & 3.63 & 5.8 \\
\hline $\begin{array}{l}\text { Proceedings of the Royal Society of } \\
\text { London. Biological Sciences }\end{array}$ & v. 1, fisrt page 25 , last page 31 & $0962-8452$ & 3.612 & 6.7 \\
\hline $\begin{array}{l}\text { Proceedings of the Royal Society of } \\
\text { London. Biological Sciences }\end{array}$ & v. 272, fistt page 1941, last page 1947 & $0962-8452$ & 3.612 & 6.7 \\
\hline Biochemical Pharmacology & v. 72, fistt page 377, last page 384 & 0006-2952 & 3.581 & 8.4 \\
\hline European Journal of Biochemistry & v. 271 , fisrt page 4270 , last page 4274 & 0014-2956 & 3.579 & 9.6 \\
\hline European Journal of Biochemistry & v. 271, fisrt page 845, last page 854 & 0014-2956 & 3.579 & 9.6 \\
\hline Genomics (San Diego) & v. 88, fisrt page 698, last page 705 & $0888-7543$ & 3.558 & 8.6 \\
\hline Applied and Environmental Microbiology & v. 1 , fisrt page 1766, last page 1770 & $0099-2240$ & 3.532 & 7.4 \\
\hline Applied and Environmental Microbiology & v. 71 , fisrt page 3276 , last page 3284 & $0099-2240$ & 3.532 & 7.4 \\
\hline Applied and Environmental Microbiology & v. 72 , fisrt page 3103 , last page 3110 & $0099-2240$ & 3.532 & 7.4 \\
\hline Molecular Phylogenetics and Evolution & v. 01, fisrt page 152, last page 163 & $1055-7903$ & 3.528 & 4.6 \\
\hline Molecular Phylogenetics and Evolution & v. 30 , fisrt page 308 , last page 324 & $1055-7903$ & 3.528 & 4.6 \\
\hline Molecular Phylogenetics and Evolution & v. 32 , fisrt page 152 , last page 163 & $1055-7903$ & 3.528 & 4.6 \\
\hline Molecular Phylogenetics and Evolution & v. 32 , fisrt page 309 , last page 323 & $1055-7903$ & 3.528 & 4.6 \\
\hline Molecular Phylogenetics and Evolution & v. 34 , fisrt page 601 , last page 615 & $1055-7903$ & 3.528 & 4.6 \\
\hline Molecular Phylogenetics and Evolution & v. 35 , fisrt page 637 , last page 645 & $1055-7903$ & 3.528 & 4.6 \\
\hline Molecular Phylogenetics and Evolution & v. 36 , fisrt page 623 , last page 640 & $1055-7903$ & 3.528 & 4.6 \\
\hline Molecular Phylogenetics and Evolution & v. 38 , fisrt page 603 , last page 620 & $1055-7903$ & 3.528 & 4.6 \\
\hline Molecular Phylogenetics and Evolution & v. 38 , fisrt page 719 , last page 730 & $1055-7903$ & 3.528 & 4.6 \\
\hline Biology of Reproduction & v. 73 , fisrt page 891 , last page 898 & $0006-3363$ & 3.498 & 6.8 \\
\hline Biology of Reproduction & v. 75 , fisrt page 370 , last page 379 & $0006-3363$ & 3.498 & 6.8 \\
\hline Journal of Structural Biology & v. 145 , fistt page 216 , last page 225 & $1047-8477$ & 3.496 & 6.2 \\
\hline Ecological Applications & v. 14 , fisrt page 72 , last page 88 & $1051-0761$ & 3.47 & 6.7 \\
\hline Ecological Applications & v. 14 , Suppl., fisrt page 150, last page 163 & $1051-0761$ & 3.47 & 6.7 \\
\hline Ecological Applications & v. 15, fisrt page 1664, last page 1678 & $1051-0761$ & 3.47 & 6.7 \\
\hline Diversity and Distributions & v. 12 , fisrt page 157 , last page 164 & $1366-9516$ & 3.441 & 3.4 \\
\hline Functional Ecology & v. 19 , fisrt page 932 , last page 940 & $0269-8463$ & 3.417 & 8.2 \\
\hline FEBS Letters & v. 10, fist page 192, last page 202 & $0014-5793$ & 3.372 & 7.7 \\
\hline FEBS Letters & v. 273 , fistt page 3962 , last page 3974 & $0014-5793$ & 3.372 & 7.7 \\
\hline FEBS Letters & v. 579 , fisrt page 4961 , last page 4965 & $0014-5793$ & 3.372 & 7.7 \\
\hline Ecography (Copenhagen) & v. 27, fistt page 253, last page 267 & $0906-7590$ & 3.34 & 5.6 \\
\hline Ecography (Copenhagen) & v. 29 , fisrt page 129 , last page 151 & $0906-7590$ & 3.34 & 5.6 \\
\hline International Journal for Parasitology & v. 34 , fisrt page 963 , last page 970 & $0020-7519$ & 3.337 & 6.3 \\
\hline Oecologia & v. 1 , fisrt page 468 , last page 479 & $0029-8549$ & 3.333 & 9.6 \\
\hline Oecologia & v. 140 , fistt page 252 , last page 260 & $0029-8549$ & 3.333 & 9.6 \\
\hline Oecologia & v. 141 , fisrt page 596, last page 614 & 0029-8549 & 3.333 & 9.6 \\
\hline Oecologia & v. 145 , fisrt page 454 , last page 461 & 0029-8549 & 3.333 & 9.6 \\
\hline Oecologia & v. 145 , fistt page 87 , last page 99 & 0029-8549 & 3.333 & 9.6 \\
\hline Plant and Cell Physiology & v. 47 , fisrt page 346 , last page 354 & $0032-0781$ & 3.324 & 6.2 \\
\hline Global Ecology and Biogeography & v. 13 , fisrt page 439 , last page 444 & $1466-822 x$ & 3.314 & 4.3 \\
\hline Global Ecology and Biogeography & v. 13, fisrt page 85, last page 92 & $1466-822 x$ & 3.314 & 4.3 \\
\hline Evolution \& Development & v. 7 , fistt page 429 , last page 439 & $1520-541 X$ & 3.293 & 3.6 \\
\hline Cancer Letters & v. 219 , fisrt page 49 , last page 55 & 0304-3835 & 3.277 & 5.3 \\
\hline $\begin{array}{l}\text { European Journal of Pharmaceutics and } \\
\text { Biopharmaceutics, Bioinfo }\end{array}$ & v. 64 , fisrt page 154 , last page 160 & $0939-6411$ & 3.185 & 4.7 \\
\hline Chemical Research in Toxicology & v. 19, fisrt page 351, last page 355 & $0893-228 X$ & 3.162 & 6.2 \\
\hline Vaccine (Guildford) & v. 22 , fisrt page 2234 , last page 2243 & $0264-410 \mathrm{X}$ & 3.159 & 4.8 \\
\hline
\end{tabular}




\begin{tabular}{|c|c|c|c|c|}
\hline Journal & Paper info. & ISSN & IF & Half-Life \\
\hline FEMS Microbiology Ecology & v. 49 , fisrt page 295 , last page 305 & 0168-6496 & 3.157 & 5.3 \\
\hline FEMS Microbiology Ecology & v. 56 , fisrt page 207 , last page 218 & 0168-6496 & 3.157 & 5.3 \\
\hline Remote Sensing of Environment & v. 95 , fisrt page 125 , last page 137 & 0034-4257 & 3.064 & 6.6 \\
\hline Chromosome Research & v. 13 , fisrt page 339 , last page 347 & 0967-3849 & 3.057 & 5.1 \\
\hline Chromosome Research & v. 13 , fisrt page 651 , last page 663 & 0967-3849 & 3.057 & 5.1 \\
\hline Journal of Evolutionary Biology & v. 17 , fisrt page 144 , last page 155 & $1010-061 X$ & 2.97 & 4.7 \\
\hline Journal of Evolutionary Biology & v. 19 , fisrt page 1139 , last page 1148 & $1010-061 X$ & 2.97 & 4.7 \\
\hline American Journal of Botany & v. 91 , fisrt page 1105 , last page 1114 & $0002-9122$ & 2.969 & 9.5 \\
\hline American Journal of Botany & v. 91 , fisrt page 1409 , last page 1418 & $0002-9122$ & 2.969 & 9.5 \\
\hline American Journal of Botany & v. 91 , fisrt page 149 , last page 157 & $0002-9122$ & 2.969 & 9.5 \\
\hline American Journal of Botany & v. 91 , fisrt page 707 , last page 723 & $0002-9122$ & 2.969 & 9.5 \\
\hline American Journal of Botany & v. 92, fist page 1513, last page 1519 & 0002-9122 & 2.969 & 9.5 \\
\hline American Journal of Botany & v. 92, fisrt page 456 , last page 461 & 0002-9122 & 2.969 & 9.5 \\
\hline American Journal of Botany & v. 92, fisrt page 613 , last page 624 & 0002-9122 & 2.969 & 9.5 \\
\hline American Journal of Botany & v. 92 , fisrt page 674 , last page 681 & $0002-9122$ & 2.969 & 9.5 \\
\hline American Journal of Botany & v. 93 , fisrt page 288 , last page 303 & $0002-9122$ & 2.969 & 9.5 \\
\hline American Journal of Botany & v. 93 , fisrt page 304 , last page 318 & $0002-9122$ & 2.969 & 9.5 \\
\hline Aquatic Toxicology & v. 67, fisrt page 57 , last page 73 & $0166-445 X$ & 2.964 & 6.1 \\
\hline Aquatic Toxicology & v. 70 , fisrt page 1 , last page 10 & $0166-445 X$ & 2.964 & 6.1 \\
\hline Aquatic Toxicology & v. 72 , fisrt page 339 , last page 349 & $0166-445 X$ & 2.964 & 6.1 \\
\hline Aquatic Toxicology & v. 72, fisrt page 67, last page 82 & $0166-445 X$ & 2.964 & 6.1 \\
\hline Aquatic Toxicology & v. 79 , fistt page 1 , last page 8 & $0166-445 X$ & 2.964 & 6.1 \\
\hline Molecular Plant Pathology & v. 5 , fisrt page 453 , last page 464 & $1464-6722$ & 2.963 & 3.6 \\
\hline Planta (Berlin) & v. 223 , fistt page 306 , last page 314 & 0032-0935 & 2.963 & 8.7 \\
\hline Reproduction (Cambridge) & v. 128 , fistt page 73 , last page 86 & $1470-1626$ & 2.958 & 3.8 \\
\hline Experimental Gerontology & v. 39 , fisrt page 767 , last page 773 & $0531-5565$ & 2.93 & 5 \\
\hline $\begin{array}{l}\text { Medicine and Science in Sports and } \\
\text { Exercise }\end{array}$ & v. 38 , fisrt page 411 , last page 417 & 0195-9131 & 2.909 & 7.7 \\
\hline Antiviral Research & v. 64 , fisrt page 69 , last page 76 & 0166-3542 & 2.878 & 5.1 \\
\hline Journal of Biogeography & v. 31 , fisrt page 1093, last page 1106 & 0305-0270 & 2.878 & 6.1 \\
\hline Journal of Biogeography & v. 31 , fisrt page 943 , last page 957 & 0305-0270 & 2.878 & 6.1 \\
\hline Journal of Biogeography & v. 32 , fisrt page 1284 , last page 1286 & 0305-0270 & 2.878 & 6.1 \\
\hline Journal of Biogeography & v. 33, fisrt page 1334, last page 1347 & $0305-0270$ & 2.878 & 6.1 \\
\hline Journal of Biogeography & v. 33 , fisrt page 1348, last page 1356 & 0305-0270 & 2.878 & 6.1 \\
\hline Journal of Biogeography & v. 33 , fisrt page 361 , last page 375 & $0305-0270$ & 2.878 & 6.1 \\
\hline Journal of Biogeography & v. 33 , fisrt page 770 , last page 780 & $0305-0270$ & 2.878 & 6.1 \\
\hline Journal of Biogeography & v. 33, fisrt page 888, last page 900 & $0305-0270$ & 2.878 & 6.1 \\
\hline Journal of Biogeography & v. 8 , fisrt page 1409 , last page 1427 & $0305-0270$ & 2.878 & 6.1 \\
\hline Heredity (Edinburgh) & v. 01 , fisrt page 228 , last page 233 & 0018-067X & 2.872 & 10 \\
\hline Heredity (Edinburgh) & v. 92 , fisrt page 466 , last page 473 & 0018-067X & 2.872 & 10 \\
\hline Heredity (Edinburgh) & v. 95 , fisrt page 245 , last page 254 & 0018-067X & 2.872 & 10 \\
\hline Heredity (Edinburgh) & v. 95 , fisrt page 274 , last page 280 & 0018-067X & 2.872 & 10 \\
\hline Heredity (Edinburgh) & v. 97 , fisrt page 329, last page 335 & 0018-067X & 2.872 & 10 \\
\hline $\begin{array}{l}\text { Biochemical and Biophysical Research } \\
\text { Communications }\end{array}$ & v. 325 , fistt page 487 , last page 493 & $0006-291 X$ & 2.855 & 6.6 \\
\hline Biochemical and Biophysical Research & v. 326 , fistt page 687 , last page 694 & $0006-291 x$ & & \\
\hline Communications & & & 2.855 & 6.6 \\
\hline Biological Conservation & v. 118 , fisrt page 421 , last page 430 & 0006-3207 & 2.854 & 5.9 \\
\hline Biological Conservation & v. 124 , fistt page 253 , last page 266 & 0006-3207 & 2.854 & 5.9 \\
\hline Biological Conservation & v. 127 , fistt page 510 , last page 515 & 0006-3207 & 2.854 & 5.9 \\
\hline Biological Conservation & v. 128 , fist page 308 , last page 315 & 0006-3207 & 2.854 & 5.9 \\
\hline Biological Conservation & v. 128, fistt page 391, last page 402 & 0006-3207 & 2.854 & 5.9 \\
\hline Biological Conservation & v. 131 , fistt page 1 , last page 13 & 0006-3207 & 2.854 & 5.9 \\
\hline Biological Conservation & v. 132 , fistt page 50 , last page 60 & 0006-3207 & 2.854 & 5.9 \\
\hline Biological Conservation & v. 134 , fisrt page 106 , last page 112 & 0006-3207 & 2.854 & 5.9 \\
\hline Biological Conservation & v. 27, fisrt page 46, last page 54 & 0006-3207 & 2.854 & 5.9 \\
\hline Biological Conservation & v. I, fisrt page 431 , last page 435 & 0006-3207 & 2.854 & 5.9 \\
\hline Tetrahedron (Oxford) & v. 61 , fisrt page 10699 , last page 10740 & 0040-4020 & 2.817 & 7.7 \\
\hline Talanta (Oxford) & v. 67, fisrt page 651, last page 657 & $0039-9140$ & 2.81 & 5.3 \\
\hline Talanta (Oxford) & v. 70 , fisrt page 588 , last page 594 & $0039-9140$ & 2.81 & 5.3 \\
\hline Journal of Orthoptera Research & v. 12 , fisrt page 141 , last page 148 & $1082-6467$ & 2.784 & 7.5 \\
\hline
\end{tabular}




\begin{tabular}{|c|c|c|c|c|}
\hline Journal & Paper info. & ISSN & IF & Half-Life \\
\hline Journal of Orthoptera Research & v. 14 , fisrt page 127 , last page 135 & $1082-6467$ & 2.784 & 7.5 \\
\hline Frontiers in Bioscience & v. 9, fisrt page 1348, last page 1359 & $1093-9946$ & 2.771 & 3.8 \\
\hline Environmental Pollution (Barking) & v. 129 , fisrt page 247 , last page 255 & $0269-7491$ & 2.769 & 5.7 \\
\hline Environmental Pollution (Barking) & v. 135 , fistt page 143 , last page 154 & $0269-7491$ & 2.769 & 5.7 \\
\hline Journal of Molecular Evolution & v. 59 , fisrt page 464 , last page 477 & $0022-2844$ & 2.767 & 10 \\
\hline Annals of Human Genetics & v. 69 , fisrt page 389 , last page 400 & $0003-4800$ & 2.727 & 7.8 \\
\hline Fish \& Shellfish Immunology & v. 21 , fisrt page 242 , last page 250 & $1050-4648$ & 2.725 & 5.2 \\
\hline Gene (Amsterdam) & v. 344, fisrt page 93, last page 103 & 0378-1119 & 2.721 & 8.5 \\
\hline Gene (Amsterdam) & v. 349, fisrt page 77 , last page 85 & 0378-1119 & 2.721 & 8.5 \\
\hline Theoretical and Applied Genetics & v. 111 , fistt page 1060 , last page 1071 & $0040-5752$ & 2.715 & 7.2 \\
\hline Theoretical and Applied Genetics & v. 112, fistt page 924, last page 933 & $0040-5752$ & 2.715 & 7.2 \\
\hline Animal Behaviour & v. 73 , fisrt page 1 , last page 13 & 0003-3472 & 2.711 & 10 \\
\hline Insect Biochemistry and Molecular Biology & v. 13, fisrt page 459, last page 467 & $0965-1748$ & 2.711 & 6.3 \\
\hline Insect Biochemistry and Molecular Biology & v. 34 , fisrt page 1257 , last page 1268 & $0965-1748$ & 2.711 & 6.3 \\
\hline Insect Biochemistry and Molecular Biology & v. 34 , fisrt page 415 , last page 424 & $0965-1748$ & 2.711 & 6.3 \\
\hline Insect Biochemistry and Molecular Biology & v. 35 , fisrt page 541 , last page 552 & 0965-1748 & 2.711 & 6.3 \\
\hline Insect Biochemistry and Molecular Biology & v. 35, fisrt page 85 , last page 91 & 0965-1748 & 2.711 & 6.3 \\
\hline Peptides (New York) & v. 25, fisrt page 919 , last page 928 & 0196-9781 & 2.701 & 6.3 \\
\hline Marine Chemistry & v. 102 , fisrt page 252 , last page 266 & $0304-4203$ & 2.663 & 9.5 \\
\hline $\begin{array}{l}\text { International Journal of Systematic and } \\
\text { Evolutionary Microbiology }\end{array}$ & v. 54 , fisrt page 1323 , last page 1328 & $1466-5026$ & 2.662 & 3.7 \\
\hline International Journal of Systematic and & v. 56, fisrt page 1931, last page 1937 & $1466-5026$ & L.TuL & 0.1 \\
\hline Evolutionary Microbiology & & & 2.662 & 3.7 \\
\hline Environmental and Molecular Mutagenesis & v. 44 , fisrt page 459 , last page 468 & 0893-6692 & 2.653 & 7.2 \\
\hline Journal of Chromatography B & v. 833 , fistt page 135 , last page 140 & $1570-0232$ & 2.647 & 5.8 \\
\hline Molecular and Biochemical Parasitology & v. 136 , fist page 87 , last page 100 & 0166-6851 & 2.641 & 7.6 \\
\hline Insect Molecular Biology & v. 13, fisrt page 55 , last page 63 & 0962-1075 & 2.639 & 5.4 \\
\hline Insect Molecular Biology & v. 15 , fisrt page 703 , last page 714 & 0962-1075 & 2.639 & 5.4 \\
\hline Journal of Experimental Biology & v. 207, fisrt page 1379, last page 1385 & 0022-0949 & 2.631 & 7.2 \\
\hline Journal of Experimental Biology & v. 207, fisrt page 307 , last page 318 & 0022-0949 & 2.631 & 7.2 \\
\hline Journal of Experimental Biology & v. 207 , fisrt page 579 , last page 585 & 0022-0949 & 2.631 & 7.2 \\
\hline Journal of Experimental Biology & v. 208 , fisrt page 3747 , last page 3759 & 0022-0949 & 2.631 & 7.2 \\
\hline Journal of Experimental Biology & v. 209, fisrt page 1185 , last page 1196 & 0022-0949 & 2.631 & 7.2 \\
\hline Journal of Experimental Biology & v. 209 , fisrt page 2628 , last page 2636 & 0022-0949 & 2.631 & 7.2 \\
\hline Journal of Experimental Biology & v. 52, fist page 397 , last page 400 & 0022-0949 & 2.631 & 7.2 \\
\hline Atmospheric Environment & v. 40, fisrt page 5701 , last page 5711 & $1352-2310$ & 2.63 & 6 \\
\hline Bioorganic \& Medicinal Chemistry & v. 13 , fist page 4184 , last page 4190 & 0968-0896 & 2.624 & 3.6 \\
\hline Bioorganic \& Medicinal Chemistry & v. 13 , fisrt page 4499 , last page 4506 & 0968-0896 & 2.624 & 3.6 \\
\hline Soil Biology \& Biochemistry & v. 38, fisrt page 1989, last page 1993 & 0038-0717 & 2.623 & 8.7 \\
\hline Geophysical Research Letters & v. 33 , fisrt page 26592 , last page 26595 & 0094-8276 & 2.602 & 5.6 \\
\hline Journal of Phycology & v. 41, fisrt page 1162, last page 1168 & $0022-3646$ & 2.58 & 8.8 \\
\hline Journal of Phycology & v. 42 , fisrt page 1271 , last page 1289 & $0022-3646$ & 2.58 & 8.8 \\
\hline Systematic Entomology & v. 29 , fisrt page 260 , last page 275 & 0307-6970 & 2.571 & 7.6 \\
\hline Systematic Entomology & v. 29 , fisrt page 69 , last page 99 & 0307-6970 & 2.571 & 7.6 \\
\hline Systematic Entomology & v. 30 , fisrt page 480 , last page 496 & 0307-6970 & 2.571 & 7.6 \\
\hline Systematic Entomology & v. 31 , fisrt page 508 , last page 516 & 0307-6970 & 2.571 & 7.6 \\
\hline Molecular Genetics and Genomics & v. 272 , fisrt page 194 , last page 203 & $1617-4615$ & 2.552 & 3.6 \\
\hline Animal Cognition & v. 9, fisrt page 229 , last page 233 & $1435-9448$ & 2.54 & 4.3 \\
\hline Bioorganic \& Medicinal Chemistry Letters & v. 14, fisrt page 1573, last page 1575 & 0960-894X & 2.538 & 3.8 \\
\hline Radiocarbon & v. B223/224, fisrt page 528, last page 534 & 0033-8222 & 2.538 & 8.8 \\
\hline Biogeochemistry & v. 79 , fisrt page 239 , last page 250 & $0168-2563$ & 2.536 & 6.9 \\
\hline Biogeochemistry & v. 79, fisrt page 3 , last page 24 & $0168-2563$ & 2.536 & 6.9 \\
\hline Biological Invasions & v. 5 , fist page 1 , last page 10 & $1387-3547$ & 2.531 & 5 \\
\hline Biological Invasions & v. 8 , fist page 677 , last page 681 & $1387-3547$ & 2.531 & 5 \\
\hline European Journal of Pharmacology & v. 2, fist page 155 , last page 159 & 0014-2999 & 2.522 & 7.6 \\
\hline European Journal of Pharmacology & v. 525 , fisrt page 24 , last page 31 & 0014-2999 & 2.522 & 7.6 \\
\hline European Journal of Pharmacology & v. 546 , fisrt page 182 , last page 188 & 0014-2999 & 2.522 & 7.6 \\
\hline Taxon & v. 53, fisrt page 1007, last page 1018 & 0040-0262 & 2.519 & 9.2 \\
\hline Taxon & v. 53, fisrt page 182, last page 184 & 0040-0262 & 2.519 & 9.2 \\
\hline Taxon & v. 53 , fisrt page 219 , last page 221 & 0040-0262 & 2.519 & 9.2 \\
\hline Taxon & v. 53, fisrt page 55 , last page 60 & $0040-0262$ & 2.519 & 9.2 \\
\hline
\end{tabular}




\begin{tabular}{|c|c|c|c|c|}
\hline Journal & Paper info. & ISSN & IF & Half-Life \\
\hline Taxon & v. 53 , fisrt page 551 , last page 553 & $0040-0262$ & 2.519 & 9.2 \\
\hline Taxon & v. 53, fisrt page 99, last page 107 & 0040-0262 & 2.519 & 9.2 \\
\hline Taxon & v. 54 , fisrt page 1 , last page 3 & 0040-0262 & 2.519 & 9.2 \\
\hline Taxon & v. 54 , fisrt page 1038 , last page 1040 & 0040-0262 & 2.519 & 9.2 \\
\hline Taxon & v. 54 , fisrt page 1105 , last page 1108 & 0040-0262 & 2.519 & 9.2 \\
\hline Taxon & v. 54 , fisrt page 693 , last page 700 & 0040-0262 & 2.519 & 9.2 \\
\hline Taxon & v. 54 , fisrt page 713 , last page 723 & 0040-0262 & 2.519 & 9.2 \\
\hline Taxon & v. 55, fisrt page 10 , last page 13 & 0040-0262 & 2.519 & 9.2 \\
\hline Taxon & v. 55 , fisrt page 119 , last page 124 & 0040-0262 & 2.519 & 9.2 \\
\hline Taxon & v. 55 , fisrt page 421 , last page 430 & 0040-0262 & 2.519 & 9.2 \\
\hline Taxon & v. 55 , fisrt page 469 , last page 482 & 0040-0262 & 2.519 & 9.2 \\
\hline Taxon & v. 55, fistt page 965, last page 972 & 0040-0262 & 2.519 & 9.2 \\
\hline Tetrahedron Letters & v. 47 , fisrt page 5135 , last page 5137 & 0040-4039 & 2.509 & 8.3 \\
\hline Toxicon & v. 43, fisrt page 287 , last page 294 & 0041-0101 & 2.509 & 7.1 \\
\hline Toxicon & v. 44 , fisrt page 829 , last page 835 & 0041-0101 & 2.509 & 7.1 \\
\hline Toxicon & v. 45 , fisrt page 901 , last page 909 & 0041-0101 & 2.509 & 7.1 \\
\hline Toxicon & v. 46 , fisrt page 108 , last page 110 & 0041-0101 & 2.509 & 7.1 \\
\hline Toxicon & v. 46 , fisrt page 218 , last page 221 & 0041-0101 & 2.509 & 7.1 \\
\hline Toxicon & v. 46 , fisrt page 595 , last page 599 & 0041-0101 & 2.509 & 7.1 \\
\hline Toxicon & v. 46 , fisrt page 641 , last page 650 & 0041-0101 & 2.509 & 7.1 \\
\hline Toxicon & v. 47 , fisrt page 150 , last page 155 & 0041-0101 & 2.509 & 7.1 \\
\hline Toxicon & v. 47 , fisrt page 774 , last page 779 & 0041-0101 & 2.509 & 7.1 \\
\hline Toxicon & v. 47 , fisrt page 838 , last page 843 & 0041-0101 & 2.509 & 7.1 \\
\hline Toxicon & v. 48 , fisrt page 1060, last page 1067 & 0041-0101 & 2.509 & 7.1 \\
\hline Toxicon & v. 48 , fisrt page 239 , last page 245 & 0041-0101 & 2.509 & 7.1 \\
\hline Toxicon & v. 48 , fisrt page 529 , last page 535 & 0041-0101 & 2.509 & 7.1 \\
\hline Freshwater Biology & v. 50, fisrt page 1391, last page 1403 & 0046-5070 & 2.502 & 7.3 \\
\hline General and Comparative Endocrinology & v. 139 , fistt page 137, last page 142 & 0016-6480 & 2.487 & 8.9 \\
\hline Harmful Algae & v. 4 , fisrt page 743 , last page 753 & $1568-9883$ & 2.46 & 2.9 \\
\hline Harmful Algae & v. 5 , fisrt page 36 , last page 44 & $1568-9883$ & 2.46 & 2.9 \\
\hline Climatic Change & v. 71 , fisrt page 267 , last page 276 & 0165-0009 & 2.459 & 6.7 \\
\hline Water Research & v. 38 , fisrt page 1439 , last page 1448 & 0043-1354 & 2.459 & 7 \\
\hline International Microbiology & v. 7, fisrt page 213, last page 218 & $1139-6709$ & 2.455 & 3.8 \\
\hline Annals of Botany & v. 28, fisrt page 213 , last page 217 & $0305-7364$ & 2.448 & 8 \\
\hline Annals of Botany & v. 30, fisrt page 1045, last page 1056 & $0305-7364$ & 2.448 & 8 \\
\hline Annals of Botany & v. 93, fisrt page 39 , last page 51 & $0305-7364$ & 2.448 & 8 \\
\hline Annals of Botany & v. 93 , fisrt page 499 , last page 505 & $0305-7364$ & 2.448 & 8 \\
\hline Annals of Botany & v. 93 , fisrt page 609, last page 613 & 0305-7364 & 2.448 & 8 \\
\hline Annals of Botany & v. 93 , fisrt page 755 , last page 762 & 0305-7364 & 2.448 & 8 \\
\hline Annals of Botany & v. 94 , fisrt page 1 , last page 9 & $0305-7364$ & 2.448 & 8 \\
\hline Annals of Botany & v. 94 , fisrt page 305 , last page 310 & $0305-7364$ & 2.448 & 8 \\
\hline Annals of Botany & v. 94, fisrt page 33 , last page 41 & $0305-7364$ & 2.448 & 8 \\
\hline Annals of Botany & v. 94 , fisrt page 365 , last page 376 & $0305-7364$ & 2.448 & 8 \\
\hline Annals of Botany & v. 94 , fisrt page 733 , last page 740 & $0305-7364$ & 2.448 & 8 \\
\hline Annals of Botany & v. 94 , fisrt page 819 , last page 830 & $0305-7364$ & 2.448 & 8 \\
\hline Annals of Botany & v. 95 , fisrt page 1 , last page 3 & $0305-7364$ & 2.448 & 8 \\
\hline Annals of Botany & v. 95, fisrt page 1171 , last page 1177 & $0305-7364$ & 2.448 & 8 \\
\hline Annals of Botany & v. 95, fisrt page 271 , last page 276 & $0305-7364$ & 2.448 & 8 \\
\hline Annals of Botany & v. 95 , fisrt page 445 , last page 455 & $0305-7364$ & 2.448 & 8 \\
\hline Annals of Botany & v. 95, fisrt page 799, last page 806 & $0305-7364$ & 2.448 & 8 \\
\hline Annals of Botany & v. 96 , fisrt page 1191 , last page 1198 & $0305-7364$ & 2.448 & 8 \\
\hline Annals of Botany & v. 96, fisrt page 191, last page 200 & $0305-7364$ & 2.448 & 8 \\
\hline Annals of Botany & v. 96 , fisrt page 387 , last page 397 & $0305-7364$ & 2.448 & 8 \\
\hline Annals of Botany & v. 96 , fisrt page 435 , last page 444 & $0305-7364$ & 2.448 & 8 \\
\hline Annals of Botany & v. 97 , fisrt page 277 , last page 287 & $0305-7364$ & 2.448 & 8 \\
\hline Annals of Botany & v. 97 , fisrt page 357 , last page 369 & $0305-7364$ & 2.448 & 8 \\
\hline Annals of Botany & v. 97, fisrt page 389 , last page 403 & $0305-7364$ & 2.448 & 8 \\
\hline Annals of Botany & v. 98 , fisrt page 1207 , last page 1213 & $0305-7364$ & 2.448 & 8 \\
\hline Annals of Botany & v. 98, fisrt page 213 , last page 217 & $0305-7364$ & 2.448 & 8 \\
\hline Biological Journal of the Linnean Society & v. 144 , fist page 543 , last page 557 & $0024-4066$ & 2.445 & 7.5 \\
\hline
\end{tabular}




\begin{tabular}{l}
\hline Journal \\
\hline Biological Journal of the Linnean Society \\
Biological Journal of the Linnean Society \\
Biological Journal of the Linnean Society \\
Biological Journal of the Linnean Society \\
Chemosphere (Oxford) \\
Chemosphere (Oxford) \\
Chemosphere (Oxford) \\
Chemosphere (Oxford) \\
Chemosphere (Oxford) \\
Chemosphere (Oxford) \\
Applied Microbiology and Biotechnology \\
Applied Microbiology and Biotechnology \\
Physical Review E \\
Physical Review. E, Statistical Physics, \\
Plasmas, Fluids, and Related \\
Interdisciplinary Topics (Online)
\end{tabular}

Pharmacological Research

Pharmacological Research

Pharmacological Research

Journal of Natural Products

Journal of Natural Products

Journal of Natural Products

Journal of Natural Products

Journal of Natural Products

Journal of Natural Products

Journal of Natural Products

Journal of Natural Products

Journal of Natural Products

Journal of Natural Products

Phytochemistry

Phytochemistry

Phytochemistry

Phytochemistry

Phytochemistry

Phytochemistry

Phytochemistry

Phytochemistry

Phytochemistry, Estados Unidos

Food and Chemical Toxicology

Food and Chemical Toxicology

Life Sciences

Life Sciences

Life Sciences

Journal of Vegetation Science

Journal of Vegetation Science

Science of the Total Environment

Science of the Total Environment

Journal of Electroanalytical Chemistry

(Amsterdam)

Zoologica Scripta

Sensors and Actuators. B, Chemical

Journal of Agricultural and Food Chemistry Journal of Agricultural and Food Chemistry Journal of Agricultural and Food Chemistry Journal of Agricultural Science

Quaternary Research

Behavioral Ecology and Sociobiology

Behavioral Ecology and Sociobiology

Fungal Diversity

Fungal Diversity v. 85 , fist page 13 , last page 26

v. 88 , fisrt page 611 , last page 630

v. 89 , fisrt page 383 , last page 396

v. 89 , fisrt page 91 , last page 98

v. 54, fisrt page 1613, last page 1618

v. 58 , fisrt page 715 , last page 733

v. 65 , fisrt page 2477 , last page 2484

v. 65 , fisrt page 560 , last page 566

v. 66 , fisrt page 1310 , last page 1314

v. 66 , fist page 1367 , last page 1374

v. 38, fisrt page 2434, last page 2477

v. 67 , fisrt page 577 , last page 591

v. 71 , fisrt page 1 , last page 4

v. 74 , fisrt page 1 , last page 8

ISSN

0024-4066

0024-4066

0024-4066

0024-4066

$0045-6535$

0045-6535

0045-6535

0045-6535

0045-6535

0045-6535

0175-7598

0175-7598

1539-3755

1095-3787

IF Half-Life

$2.445 \quad 7.5$

$2.445 \quad 7.5$

$2.445 \quad 7.5$

$2.445 \quad 7.5$

$2.442 \quad 5.4$

$2.442 \quad 5.4$

$2.442 \quad 5.4$

$2.442 \quad 5.4$

$2.442 \quad 5.4$

$2.442 \quad 5.4$

$2.441 \quad 6.4$

$2.441 \quad 6.4$

$2.438 \quad 5.3$

v. 49 , fisrt page 245 , last page 248

v. 49 , fisrt page 449 , last page 453

v. 51 , fisrt page 229 , last page 233

v. 67 , fisrt page 1580, last page 1583

v. 67, fist page 1685, last page 1689

v. 67 , fist page 1783, last page 1788

v. 67 , fist page 1879 , last page 1881

v. 67 , fist page 510 , last page 522

v. 67 , fisrt page 809 , last page 910

v. 68, fisrt page 1290, last page 1292

v. 68 , fisrt page 423 , last page 426

v. 69 , fist page 1113 , last page 1116

v. 69 , fist page 1606 , last page 1610

v. 65 , fisrt page 449 , last page 454

v. 66, fisrt page 1927, last page 1932

v. 66 , fisrt page 2363 , last page 2367

v. 66 , fisrt page 573 , last page 579

v. 66 , fisrt page 929 , last page 934

v. 67 , fist page 1331, last page 1335

v. 67 , fisrt page 2009 , last page 2016

v. 67 , fisrt page 277 , last page 285

v. 67, fisrt page 1964, last page 1969

v. 44, fisrt page 388, last page 392

v. 44 , fisrt page 425 , last page 435

v. 78, fist page 2409, last page 2417

v. 79, fist page 1692, last page 1701

v. 79 , fist page 2099, last page 2104

v. 15 , fist page 41 , last page 48

v. 172 , fisrt page 121 , last page 131

v. 364, fisrt page 188, last page 199

v. 368 , fisrt page 344 , last page 351

v. 589 , fisrt page 70 , last page 81

v. 33, fisrt page 551, last page 586

v. 114 , fisrt page 1019, last page 1027

v. 3 \& 4, fisrt page 33 , last page 35

v. 52 , fisrt page 2533 , last page 2540

v. 52, fisrt page 7544, last page 7554

v. 145, fist page 01, last page 09

v. 61 , fisrt page 289 , last page 300

v. 58 , fisrt page 27 , last page 35

v. 60 , fisrt page 815 , last page 825

v. 18 , fisrt page 9 , last page 14

v. 21 , fisrt page 11 , last page 18
0048-9697

$1572-6657$

0300-3256

0925-4005

0021-8561

0021-8561

0021-8561

0021-8596

0033-5894

0340-5443

0340-5443

$1560-2745$

1560-2745
2.438

2.421

2.421

2.421

2.418

2.418

2.418

2.418

2.418

2.418

2.418

2.418

2.418

2.418

2.417

2.417

2.417

2.417

2.417

2.417

2.417

2.417

2.417

2.393

2.393

2.389

2.389

2.389

2.382

2.382

2.359

2.359

2.339

2.338

5.3

4.9

4.9

4.9

6.7

6.7

6.7

6.7

6.7

6.7

6.7

6.7

6.7

6.7

10

10

10

10

10

10

10

10

10

6.6

6.6

7.7

7.7

7.7

7.9

7.9

7.1

7.1

10

7.9

5.5

2.331

2.322

2.322

2.322

2.322

2.319

2.316

2.316

2.297

2.297 


\begin{tabular}{|c|c|c|c|c|}
\hline Journal & Paper info. & ISSN & $\mathrm{IF}$ & Half-Life \\
\hline Fungal Diversity & v. 27, fisrt page 1, last page 10 & $1560-2745$ & 2.297 & 2.8 \\
\hline Tree Physiology & v. 24 , fistt page 1119 , last page 1127 & 0829-318X & 2.297 & 7 \\
\hline Tree Physiology & v. 24, fistt page 891 , last page 899 & $0829-318 x$ & 2.297 & 7 \\
\hline Tree Physiology & v. 25 , fisrt page 251 , last page 255 & $0829-318 x$ & 2.297 & 7 \\
\hline Tree Physiology & v. 27 , fisrt page 551 , last page 559 & $0829-318 x$ & 2.297 & 7 \\
\hline Marine Ecology. Progress Series & v. 279 , fisrt page 193 , last page 200 & $0171-8630$ & 2.286 & 8.6 \\
\hline Marine Ecology. Progress Series & v. 283 , fisrt page 113 , last page 125 & $0171-8630$ & 2.286 & 8.6 \\
\hline Marine Ecology. Progress Series & v. 291 , fisrt page 35 , last page 42 & $0171-8630$ & 2.286 & 8.6 \\
\hline Marine Ecology. Progress Series & v. 300 , fisrt page 229 , last page 240 & $0171-8630$ & 2.286 & 8.6 \\
\hline Marine Ecology. Progress Series & v. 307 , fisrt page 21 , last page 36 & $0171-8630$ & 2.286 & 8.6 \\
\hline Marine Ecology. Progress Series & v. 308 , fisrt page 37 , last page 48 & 0171-8630 & 2.286 & 8.6 \\
\hline Marine Ecology. Progress Series & v. 313 , fisrt page 295 , last page 304 & $0171-8630$ & 2.286 & 8.6 \\
\hline Functional Plant Biology & v. 33 , fisrt page 1103 , last page 1113 & $1445-4408$ & 2.272 & 3.2 \\
\hline Ethology & v. 111 , fist page 1116 , last page 1125 & 0179-1613 & 2.245 & 8.2 \\
\hline Nutrition & v. 22, fisrt page 197, last page 205 & 0899-9007 & 2.229 & 5.7 \\
\hline Acta Tropica & v. 90, fisrt page 97, last page 106 & $0001-706 X$ & 2.211 & 5.4 \\
\hline Acta Tropica & v. 98 , fisrt page 15 , last page 24 & $0001-706 x$ & 2.211 & 5.4 \\
\hline Acta Tropica & v. 99 , fisrt page 252 , last page 259 & 0001-706X & 2.211 & 5.4 \\
\hline Aquatic Microbial Ecology & v. 40 , fisrt page 77 , last page 83 & 0948-3055 & 2.209 & 5.5 \\
\hline Journal of Applied Microbiology & v. 100 , fisrt page 641 , last page 647 & $1364-5072$ & 2.206 & 5 \\
\hline Journal of Applied Microbiology & v. 98 , fisrt page 667 , last page 675 & $1364-5072$ & 2.206 & 5 \\
\hline Coral Reefs & v. 24 , fisrt page 191 , last page 193 & $0722-4028$ & 2.203 & 7.8 \\
\hline Coral Reefs & v. 25 , fisrt page 212 , last page 214 & $0722-4028$ & 2.203 & 7.8 \\
\hline Coral Reefs & v. 25 , fisrt page 212 , last page 215 & $0722-4028$ & 2.203 & 7.8 \\
\hline Coral Reefs & v. 25 , fisrt page 58 , last page 60 & $0722-4028$ & 2.203 & 7.8 \\
\hline Environmental Toxicology and Chemistry & v. 23 , fisrt page 388 , last page 395 & $0730-7268$ & 2.202 & 6.6 \\
\hline Environmental Toxicology and Chemistry & v. 23, fisrt page 883 , last page 889 & $0730-7268$ & 2.202 & 6.6 \\
\hline Plant Pathology & v. 53 , fisrt page 334 , last page 340 & $0032-0862$ & 2.198 & 8 \\
\hline Plant Pathology & v. 55, fisrt page 187, last page 193 & $0032-0862$ & 2.198 & 8 \\
\hline Photosynthesis Research & v. 90, fisrt page 79 , last page 90 & 0166-8595 & 2.193 & 7.8 \\
\hline Animal Reproduction Science & v. 91 , fisrt page 173 , last page 179 & $0378-4320$ & 2.186 & 6.4 \\
\hline Animal Reproduction Science & v. 91 , fisrt page 307 , last page 316 & $0378-4320$ & 2.186 & 6.4 \\
\hline Growth Hormone \& IGF Research & v. 16 , fisrt page 326 , last page 331 & $1096-6374$ & 2.183 & 3.7 \\
\hline Bioresource Technology & v. 91, fisrt page 69 , last page 75 & $0960-8524$ & 2.18 & 5.5 \\
\hline Bioresource Technology & v. 93, fisrt page 29, last page 35 & $0960-8524$ & 2.18 & 5.5 \\
\hline Bioresource Technology & v. 97, fisrt page 862 , last page 867 & 0960-8524 & 2.18 & 5.5 \\
\hline Physiologia Plantarum (Copenhagen) & v. 125 , fisrt page 419 , last page 429 & $0031-9317$ & 2.169 & 10 \\
\hline American Journal of Physical Anthropology & v. 123 (S38), fisrt page 200, last page 202 & $0002-9483$ & 2.136 & 10 \\
\hline American Journal of Physical Anthropology & v. 125, fisrt page 203, last page 205 & $0002-9483$ & 2.136 & 10 \\
\hline American Journal of Physical Anthropology & v. 127 , fisrt page 439 , last page 448 & $0002-9483$ & 2.136 & 10 \\
\hline American Journal of Physical Anthropology & v. 128 , fisrt page 171 , last page 184 & $0002-9483$ & 2.136 & 10 \\
\hline American Journal of Physical Anthropology & v. 129 , fisrt page 157 , last page 160 & $0002-9483$ & 2.136 & 10 \\
\hline American Journal of Physical Anthropology & v. 130 , fisrt page 227 , last page 237 & $0002-9483$ & 2.136 & 10 \\
\hline Geoderma (Amsterdam) & v. 119 , fisrt page 309 , last page 321 & 0016-7061 & 2.124 & 7.5 \\
\hline $\begin{array}{l}\text { Mutation Research. Genetic Toxicology } \\
\text { and Environmental Mutagenesis }\end{array}$ & v. 560, fisrt page 57, last page 67 & $1383-5718$ & 2.122 & 5.6 \\
\hline $\begin{array}{l}\text { Mutation Research. Genetic Toxicology } \\
\text { and Environmental Mutagenesis }\end{array}$ & v. 604 , fisrt page 71 , last page 82 & $1383-5718$ & 2.122 & 5.6 \\
\hline Journal of Hydrology (Amsterdam) & v. 331 , fisrt page 425 , last page 433 & 0022-1694 & 2.117 & 8.6 \\
\hline Microscopy \& Microanalysis & v. 11 , fisrt page 166 , last page 174 & $1431-9276$ & 2.108 & 3.7 \\
\hline JMBA Global Marine Environment & v. 72 , fisrt page 437 , last page 441 & 0141-1136 & 2.106 & 6.8 \\
\hline Marine Environmental Research & v. 58, fisrt page 1 , last page 17 & 0141-1136 & 2.106 & 6.8 \\
\hline Marine Environmental Research & v. 58 , fisrt page 263 , last page 267 & 0141-1136 & 2.106 & 6.8 \\
\hline Marine Environmental Research & v. 58 , fisrt page 625 , last page 630 & 0141-1136 & 2.106 & 6.8 \\
\hline Biotechnology Progress & v. 20 , fisrt page 1880 , last page 1884 & $8756-7938$ & 2.102 & 5.5 \\
\hline Biotechnology Progress & v. 22 , fisrt page 1637 , last page 1642 & $8756-7938$ & 2.102 & 5.5 \\
\hline $\begin{array}{l}\text { Bulletin of the American Museum of Natural } \\
\text { History }\end{array}$ & v. 285 , fisrt page 25 , last page 35 & $0003-0090$ & 2.1 & 10 \\
\hline $\begin{array}{l}\text { Bulletin of the American Museum of Natural } \\
\text { History }\end{array}$ & v. 294 , fisrt page 1 , last page 240 & 0003-0090 & 2.1 & 10 \\
\hline $\begin{array}{l}\text { Bulletin of the American Museum of Natural } \\
\text { History }\end{array}$ & v. 297 , fisrt page 01 , last page 370 & 0003-0090 & 2.1 & 10 \\
\hline
\end{tabular}


Journal

History

Neuroscience Letters

Seed Science Research

Aquaculture (Amsterdam)

Aquaculture (Amsterdam)

Aquaculture (Amsterdam)

Aquaculture (Amsterdam)

Aquaculture (Amsterdam)

Aquaculture (Amsterdam)

Aquaculture (Amsterdam)

Aquaculture (Amsterdam)

Aquaculture (Amsterdam)

Aquaculture (Amsterdam)

Aquaculture (Amsterdam)

Aquaculture (Amsterdam)

Aquaculture (Amsterdam)

Aquaculture (Amsterdam)

FEMS Microbiology Letters

FEMS Microbiology Letters

FEMS Microbiology Letters

Zoological Journal of the Linnean Society

Zoological Journal of the Linnean Society

Zoological Journal of the Linnean Society

Zoological Journal of the Linnean Society

American Journal of Medical Genetics

Photochemistry and Photobiology

Photochemistry and Photobiology

Photochemistry and Photobiology

Photochemistry and Photobiology

Photochemistry and Photobiology

Plant Biology

Plant Biology

Plant Biology

Plant Biology

Plant Biology

Plant Biology

Plant Biology

Plant Biology

Plant Biology

Plant Biology

Plant Biology, Stuttgart

Auk (Washington)

Respiratory Physiology and Neurobiology

Respiratory Physiology and Neurobiology

Arthropod Structure and Development

Medical and Veterinary Entomology

Medical and Veterinary Entomology

Journal of Pharmaceutical and Biomedical

Analysis

Continental Shelf Research

Continental Shelf Research

Transactions of the Royal Society of

Tropical Medicine and Hygiene

Marine Geology

Naturwissenschaften

Naturwissenschaften

Naturwissenschaften

Naturwissenschaften

Naturwissenschaften

Naturwissenschaften v. 374 , fisrt page 5 , last page 10

v. 14 , fisrt page 127, last page 135

v. 230 , fisrt page 197 , last page 204

v. 231 , fisrt page 37 , last page 49

v. 239 , fisrt page 199 , last page 215

v. 239 , fisrt page 307 , last page 321

v. 239 , fisrt page 497 , last page 507

v. 246 , fisrt page 405 , last page 412

v. 252 fisrt page 494, last page 502

v. 252 fisrt page 534, last page 544

v. 252, fisrt page 525 , last page 533

v. 253 , fisrt page 433 , last page 446

v. 253, fisrt page 646, last page 652

v. 254 , fisrt page 748 , last page 757

v. 258 , fisrt page 396 , last page 403

v. 260 , fisrt page 244 , last page 254

v. 240 , fisrt page 203 , last page 208

v. 241 , fisrt page 185 , last page 191

v. 246 , fisrt page 273 , last page 278

v. 140 , fisrt page 103 , last page 135

v. 144 , fisrt page 543 , last page 557

v. 145 , fisrt page 145 , last page 173

v. 147 , fisrt page 277 , last page 299

v. $132 \mathrm{~A}$, fisrt page 210 , last page 214

v. 82 , fisrt page 850 , last page 856

v. 82 , fist page 857 , last page 864

v. 82 , fisrt page 878 , last page 886

v. 82 , fisrt page 909 , last page 922

v. 82 , fisrt page 952, last page 961

v. 4 , fisrt page 1 , last page 3

v. 6 , fisrt page 491 , last page 497

v. 6 , fisrt page 506 , last page 512

v. 6 , fisrt page 755 , last page 760

v. 7 , fisrt page 1 , last page 3

v. 7, fist page 104, last page 107

v. 8, fist page 112, last page 119

v. 8 , fisrt page 143 , last page 154

v. 8, fisrt page 494, last page 502

v. 8 , fisrt page 529 , last page 534

v. 8 , fist page 430 , last page 438

v. 122 , fist page 292 , last page 305

v. 140 , fisrt page 197, last page 208

v. 144, fisrt page 191, last page 202

v. 33, fisrt page 431 , last page 442

v. 19 , fisrt page 208 , last page 218

v. 19 , fisrt page 428 , last page 441

v. 42 , fisrt page 184 , last page 191

v. 24 , fisrt page 983 , last page 1000

v. 25 , fist page 1720 , last page 1727

v. 98 , fisrt page 88 , last page 91

v. 207 , fisrt page 159 , last page 167

v. 91 , fisrt page 182, last page 185

v. 91 , fisrt page 94 , last page 96

v. 92 , fisrt page 147 , last page 150

v. 92 , fisrt page 319 , last page 323

v. 93, fisrt page 207 , last page 215

v. 93 , fisrt page 487 , last page 494

\section{3-0090}

0304-3940

$2.1 \quad 10$

0960-2585

2.092

10

$0044-8486$

2.085

2.081

0044-8486

2.081

0044-8486

2.081

0044-8486

0044-8486

0044-8486

2.081

2.081

2.081

0044-8486

0044-8486

0044-8486

0044-8486

0044-8486

0044-8486

0044-8486

0044-8486

0378-1097

0378-1097

0378-1097

0024-4082

0024-4082

0024-4082

0024-4082

0148-7299

0031-8655

0031-8655

0031-8655

0031-8655

0031-8655

1435-8603

1435-8603

1435-8603

$1435-8603$

1435-8603

$1435-8603$

$1435-8603$

1435-8603

$1435-8603$

1435-8603

$1435-8603$

0004-8038

1569-9048

1569-9048

1467-8039

0269-283X

0269-283X

0731-7085

0278-4343

0278-4343

0035-9203

0025-3227

0028-1042

0028-1042

0028-1042

0028-1042

0028-1042

0028-1042

2.081

2.081

2.081

2.081

2.081

2.081

2.081

2.068

2.068

2.068

2.066

2.066

2.066

2.066

2.063

2.061

2.061

2.061

2.061

2.061

2.059

2.059

2.059

2.059

2.059

2.059

2.059

2.059

2.059

2.059

2.059

2.056

2.049

2.049

2.033

2.033

2.033

2.032

2.03

2.03

2.03

2.029

2.021

2.021

2.021

2.021

2.021

2.021

10 
Physiological and Biochemical Zoology

Physiological and Biochemical Zoology

Parasite Immunology

Process Biochemistry

Marine Pollution Bulletin

Marine Pollution Bulletin

Marine Pollution Bulletin

Marine Pollution Bulletin

Marine Pollution Bulletin

Marine Pollution Bulletin

Marine Pollution Bulletin

Marine Pollution Bulletin

Marine Pollution Bulletin

Marine Pollution Bulletin

Marine Pollution Bulletin

Marine Pollution Bulletin

Marine Pollution Bulletin

Marine Pollution Bulletin

Marine Pollution Bulletin

Marine Pollution Bulletin

Comparative Immunology, Microbiology and Infectious Diseases

Ecotoxicology and Environmental Safety Ecotoxicology and Environmental Safety Ecotoxicology and Environmental Safety Ecotoxicology and Environmental Safety Ecotoxicology and Environmental Safety Ecotoxicology and Environmental Safety Ecotoxicology and Environmental Safety Veterinary Immunology and Immunopathology

Veterinary Immunology and Immunopathology

Cytogenetic and Genome Research

Cytogenetic and Genome Research

Cytogenetic and Genome Research

Cytogenetic and Genome Research

Cytogenetic and Genome Research

Cytogenetic and Genome Research

Cytogenetic and Genome Research

Comparative Biochemistry and Physiology.

C, Toxicology \& Pharmacology

Comparative Biochemistry and Physiology.

C, Toxicology \& Pharmacology

Comparative Biochemistry and Physiology.

C, Toxicology \& Pharmacology

Comparative Biochemistry and Physiology.

C, Toxicology \& Pharmacology

Canadian Journal of Anaesthesia

Genome (Ottawa)

Antonie van Leeuwenhoek

Ecological Entomology

Ecological Entomology

Ecological Entomology

Journal of Medical Entomology

Journal of Medical Entomology

Journal of Medical Entomology

Journal of Heredity

Journal of Heredity

Applied Soil Ecology

Applied Soil Ecology
Paper info.

v. 51, fist page 1135, last page 1147

v. 52, fisrt page 1121, last page 1127

v. 78, fisrt page 641 , last page 649

v. 79, fisrt page 593, last page 601

v. 26 , fisrt page 151 , last page 155

v. 39, fisrt page 2277 , last page 2282

v. 25, fisrt page 532, last page 539

v. 48 , fisrt page 97 , last page 107

v. 49 , fisrt page 368 , last page 370

v. 50 , fisrt page 329 , last page 331

v. 50 , fisrt page 624 , last page 637

v. 50, fisrt page 956, last page 964

v. 52, fisrt page 1090, last page 1117

v. 52 , fisrt page 1725 , last page 1734

v. 52 , fisrt page 175 , last page 183

v. 52 , fisrt page 1823, last page 1826

v. 52 , fisrt page 199 , last page 206

v. 52 , fisrt page 207 , last page 213

v. 52 , fisrt page 574 , last page 578

v. 52 , fisrt page 61 , last page 73

v. 52 , fisrt page 957 , last page 961

v. 52 , fisrt page 974 , last page 977

v. 29, fisrt page 301 , last page 314

v. 58 , fisrt page 117 , last page 125

v. 61 , fisrt page 247 , last page 255

v. 61 , fisrt page 353 , last page 360

v. 61 , fist page 361 , last page 365

v. 61 , fisrt page 398 , last page 403

v. 63 , fisrt page 306 , last page 312

v. 65 , fisrt page 201, last page 208

v. 106 , fisrt page 239 , last page 245

v. 113 , fisrt page 257 , last page 266

v. 103 , fisrt page 128 , last page 134

v. 106 , fisrt page 184 , last page 188

v. 108, fisrt page 106, last page 111

v. 108 , fisrt page 183 , last page 190

v. 109 , fisrt page 276 , last page 282

v. 110 , fisrt page 229 , last page 241

v. 115 , fisrt page 7 , last page 9

v. 137 , fisrt page 363 , last page 372

v. 138 , fist page 97 , last page 104

v. 140 , fisrt page 356 , last page 363

v. 142 , fisrt page 347 , last page 355

v. 52 , fisrt page 878 , last page 882

v. 47 , fisrt page 134 , last page 140

v. 88 , fist page 13 , last page 23

v. 25 , fisrt page 628 , last page 631

v. 30, fisrt page 201, last page 209

v. 30 , fist page 28 , last page 35

v. 41 , fist page 58 , last page 64

v. 42 , fisrt page 319 , last page 326

v. 43 , fisrt page 68 , last page 72

v. 95 , fisrt page 77 , last page 81

v. 97 , fisrt page 466 , last page 472

v. 25 , fisrt page 245 , last page 255

v. 28 , fisrt page 57 , last page 65
ISSN

0022-1910

IF Half-Life

$0022-1910$

2.019

9.5

$1522-2152$

$1522-2152$

0141-9838

$1359-5113$

0025-326X

0147-9571

0147-6513

0147-6513

0147-6513

0147-6513

0147-6513

0147-6513

0147-6513

0165-2427

0165-2427

1424-8581

1424-8581

1424-8581

1424-8581

1424-8581

1424-8581

1424-8581

1532-0456

1532-0456

1532-0456

1532-0456

0832-610X

0831-2796

0003-6072

0307-6946

0307-6946

0307-6946

0022-2585

0022-2585

0022-2585

0022-1503

0022-1503

0929-1393

0929-1393
2.019

2.011

2.011

2.009

2.008

2.007

2.007

2.007

2.007

2.007

2.007

2.007

2.007

2.007

2.007

2.007

2.007

2.007

2.007

2.007

2.007

9.5

4.4

4.4

7.7

4.6

6.6

6.6

6.6

6.6

6.6

6.6

6.6

6.6

6.6

6.6

6.6

6.6

6.6

6.6

6.6

6.6

6.8

6.7

6.7

6.7

6.7

6.7

6.7

6.7

1.994

6.9

1.994

6.9

2.7

2.7

2.7

2.7

2.7

2.7

2.7

1.993

1.993

8

1.991

8

1.991

8

1.991

1.976

8

1.972

7.4

7.5

$1.964 \quad 7.6$

$1.96 \quad 8.5$

$1.96 \quad 8.5$

$1.96 \quad 8.5$

$1.95 \quad 8.4$

$1.95 \quad 8.4$

$1.95 \quad 8.4$

$1.942 \quad 10$

$1.942 \quad 10$

$1.929 \quad 6.1$

$1.929 \quad 6.1$ 
Applied Soil Ecology

Applied Soil Ecology

Animal Conservation

Animal Conservation

Journal of Experimental Marine Biology and

Ecology

Journal of Experimental Marine Biology and

Ecology

Journal of Experimental Marine Biology and

Ecology

Journal of Experimental Marine Biology and

Ecology

Journal of Experimental Marine Biology and

Ecology

Journal of Experimental Marine Biology and

Ecology

Journal of Experimental Marine Biology and Ecology

Journal of Experimental Marine Biology and

Ecology

Journal of Experimental Marine Biology and

Ecology

Journal of Experimental Marine Biology and

Ecology

Journal of Experimental Marine Biology and

Ecology

Journal of Experimental Marine Biology and

Ecology

Journal of Experimental Marine Biology and Ecology

Journal of Fish Biology

Journal of Fish Biology

Journal of Fish Biology

Journal of Fish Biology

Journal of Fish Biology

Journal of Fish Biology

Journal of Fish Biology

Journal of Fish Biology

Journal of Fish Biology

Journal of Fish Biology

Journal of Fish Biology

Journal of Fish Biology

Journal of Fish Biology

Journal of Fish Biology

Journal of Fish Biology

Journal of Fish Biology

Journal of Fish Biology

Journal of Fish Biology

Journal of Fish Biology

Journal of Fish Biology

Apidologie (Celle)

Apidologie (Celle)

Apidologie (Celle)

Apidologie (Celle)

Apidologie (Celle)

Apidologie (Celle)

Apidologie (Celle)

Apidologie (Celle)

Apidologie (Celle)

Apidologie (Celle)

Apidologie (Celle)

Apidologie (Celle)

Apidologie (Celle)

Apidologie (Celle)

Apidologie (Celle)
Paper info.

ISSN

v. 29 , fist page 259 , last page 273

v. 31 , fisrt page 181 , last page 185

v. 7 , fisrt page 265 , last page 272

v. 8 , fisrt page 1 , last page 12

v. 1 , fist page 40 , last page 46

v. 145 , fisrt page 603 , last page 612

v. 313 , fisrt page 125 , last page 137

v. 316 , fisrt page 157, last page 165

v. 320 , fisrt page 211 , last page 223

v. 322 , fisrt page 167 , last page 176

v. 322 , fist page 177 , last page 183

v. 323 , fist page 118 , last page 137

v. 327 , fisrt page 58 , last page 69

v. 328 , fisrt page 127 , last page 135

v. 335 , fisrt page 82 , last page 90

v. 336 , fisrt page 211 , last page 220

v. 337 , fisrt page 59 , last page 64

v. 28 , fist page 1376, last page 1387

v. 64 , fist page 1419 , last page 1424

v. 64 , fist page 1439, last page 1441

v. 64 , fisrt page 794 , last page 798

v. 64, fisrt page 961, last page 969

v. 65 , fisrt page 287 , last page 292

v. 66 , fisrt page 45 , last page 72

v. 67 , fisrt page 1 , last page 13

v. 67 , fist page 1287, last page 1298

v. 67 , fist page 1446, last page 1459

v. 67 , fisrt page 1603 , last page 1618

v. 67 , fisrt page 1686, last page 1698

v. 68 , fisrt page 1319, last page 1332

v. 68 , fisrt page 196, last page 208

v. 68 , fisrt page 414 , last page 429

v. 69 , fisrt page 1 , last page 18

v. 69 , fist page 1504, last page 1512

v. 69 , fist page 1881 , last page 1884

v. 69 , fist page 233 , last page 242

v. 69 , fisrt page 318 , last page 321

v. 35 , fisrt page 3 , last page 13

v. 35 , fisrt page 359 , last page 364

v. 35 , fisrt page 37 , last page 47

v. 35 , fisrt page 513 , last page 518

v. 35 , fisrt page 539 , last page 541

v. 36 , fisrt page 285 , last page 291

v. 37 , fisrt page 121 , last page 123

v. 37 , fisrt page 144, last page 163

v. 37 , fisrt page 207 , last page 221

v. 37 , fisrt page 222 , last page 239

v. 37, fisrt page 275, last page 292

v. 37 , fisrt page 487 , last page 550

v. 37 , fisrt page 613 , last page 616

v. 37 , fisrt page 687 , last page 698

v. 37 , fist page 91 , last page 97

\begin{tabular}{llr}
\hline $0929-1393$ & 1.929 & 6.1 \\
$0929-1393$ & 1.929 & 6.1 \\
$1367-9430$ & 1.926 & 4.8 \\
$1367-9430$ & 1.926 & 4.8 \\
$0022-0981$ & 1.919 & 10 \\
$0022-0981$ & 1.919 & 10 \\
$0022-0981$ & 1.919 & 10 \\
$0022-0981$ & 1.919 & 10 \\
$0022-0981$ & 1.919 & 10 \\
$0022-0981$ & 1.919 & 10 \\
$0022-0981$ & 1.919 & 10 \\
$0022-0981$ & 1.919 & 10 \\
$0022-0981$ & 1.919 & 10
\end{tabular}

0022-0981

0022-0981

$1.919 \quad 10$

$1.919 \quad 10$

0022-0981

0022-0981

0022-1112

0022-1112

0022-1112

0022-1112

0022-1112

$0022-1112$

0022-1112

0022-1112

0022-1112

0022-1112

$0022-1112$

$0022-1112$

0022-1112

0022-1112

0022-1112

0022-1112

0022-1112

0022-1112

0022-1112

0022-1112

0044-8435

0044-8435

0044-8435

0044-8435

0044-8435

0044-8435

0044-8435

0044-8435

0044-8435

0044-8435

0044-8435

0044-8435

0044-8435

0044-8435

0044-8435

$1.919 \quad 10$

$1.919 \quad 10$

$1.919 \quad 10$

$1.919 \quad 10$

$1.919 \quad 10$

$1.919 \quad 10$

$1.919 \quad 10$

$1.919 \quad 10$

$1.919 \quad 10$

$1.919 \quad 10$

$1.919 \quad 10$

$1.919 \quad 10$

$1.919 \quad 10$

$1.919 \quad 10$

$1.919 \quad 10$

$1.919 \quad 10$

$1.919 \quad 10$

$1.919 \quad 10$

$1.919 \quad 10$

$1.919 \quad 10$

$1.919 \quad 10$

$1.906 \quad 7.5$

$1.906 \quad 7.5$

$1.906 \quad 7.5$

$1.906 \quad 7.5$

$1.906 \quad 7.5$

$1.906 \quad 7.5$

$1.906 \quad 7.5$

$1.906 \quad 7.5$

$1.906 \quad 7.5$

$1.906 \quad 7.5$

$1.906 \quad 7.5$

$1.906 \quad 7.5$

$1.906 \quad 7.5$

$1.906 \quad 7.5$

$1.906 \quad 7.5$
$1.919 \quad 10$ 


\begin{tabular}{|c|c|c|c|c|}
\hline Journal & Paper info. & ISSN & IF & Half-Life \\
\hline Theriogenology & v. 61 , fisrt page 1009 , last page 1024 & 0093-691X & 1.898 & 6.8 \\
\hline Enzyme and Microbial Technology & v. 36 , fisrt page 937 , last page 946 & 0141-0229 & 1.897 & 6.9 \\
\hline Enzyme and Microbial Technology & v. 36 , fisrt page 967 , last page 975 & 0141-0229 & 1.897 & 6.9 \\
\hline Enzyme and Microbial Technology & v. 37 , fisrt page 272 , last page 277 & 0141-0229 & 1.897 & 6.9 \\
\hline Journal of Chemical Ecology & v. 30 , fisrt page 1045 , last page 1056 & 0098-0331 & 1.896 & 9.5 \\
\hline Journal of Chemical Ecology & v. 30 , fisrt page 1771 , last page 1780 & 0098-0331 & 1.896 & 9.5 \\
\hline Journal of Chemical Ecology & v. 30, fisrt page 2023, last page 2036 & $0098-0331$ & 1.896 & 9.5 \\
\hline Journal of Chemical Ecology & v. 30, fisrt page 793, last page 804 & 0098-0331 & 1.896 & 9.5 \\
\hline Journal of Chemical Ecology & v. 31 , fisrt page 1509 , last page 1525 & 0098-0331 & 1.896 & 9.5 \\
\hline Journal of Chemical Ecology & v. 31 , fisrt page 2551 , last page 2561 & 0098-0331 & 1.896 & 9.5 \\
\hline Journal of Chemical Ecology & v. 32 , fisrt page 155 , last page 168 & 0098-0331 & 1.896 & 9.5 \\
\hline Journal of Chemical Ecology & v. 32 , fist page 1555 , last page 1564 & 0098-0331 & 1.896 & 9.5 \\
\hline Journal of Chemical Ecology & v. 32, fist page 1977, last page 1988 & 0098-0331 & 1.896 & 9.5 \\
\hline Journal of Chemical Ecology & v. 32 , fisrt page 59 , last page 70 & 0098-0331 & 1.896 & 9.5 \\
\hline Journal of Chemical Ecology & v. 32 , fisrt page 835 , last page 843 & 0098-0331 & 1.896 & 9.5 \\
\hline BioMetals (Oxford) & v. 18 , fisrt page 595, last page 602 & 0966-0844 & 1.893 & 4.9 \\
\hline Postharvest Biology and Technology & v. 33, fistt page 163 , last page 173 & 0925-5214 & 1.892 & 5.6 \\
\hline Ecological Modelling & v. 172 , fisrt page 283 , last page 306 & 0304-3800 & 1.888 & 5.9 \\
\hline Ecological Modelling & v. 188 , fisrt page 448 , last page 460 & $0304-3800$ & 1.888 & 5.9 \\
\hline Canadian Journal of Fisheries and Aquatic & v. 62 , fist page 1 , last page 10 & $0706-652 X$ & & \\
\hline $\begin{array}{l}\text { Sciences } \\
\text { Protein Expression and Purification }\end{array}$ & v. 47 fist page 614 l last page 620 & $1046-5928$ & 1.882 & $\begin{array}{r}10 \\
46\end{array}$ \\
\hline Mycological Research & v. 162 , fistt page 337 , last page 346 & 0953-7562 & $\begin{array}{r}1.867 \\
186\end{array}$ & $\begin{array}{l}4.6 \\
6.9\end{array}$ \\
\hline Plant Physiology and Biochemistry (Paris) & v. 42 , fisrt page 313 , last page 319 & $0981-9428$ & $\begin{array}{r}1.00 \\
1.847\end{array}$ & $\begin{array}{l}0.9 \\
5.7\end{array}$ \\
\hline Plant Physiology and Biochemistry (Paris) & v. 42 , fisrt page 389 , last page 394 & 0981-9428 & 1.847 & 5.7 \\
\hline Plant Physiology and Biochemistry (Paris) & v. 42 , fisrt page 411 , last page 415 & 0981-9428 & 1.847 & 5.7 \\
\hline Plant Physiology and Biochemistry (Paris) & v. 43 , fisrt page 241 , last page 248 & 0981-9428 & 1.847 & 5.7 \\
\hline Plant Physiology and Biochemistry (Paris) & v. 44 , fisrt page 420 , last page 429 & 0981-9428 & 1.847 & 5.7 \\
\hline Plant Physiology and Biochemistry (Paris) & v. 44 , fisrt page 619 , last page 627 & 0981-9428 & 1.847 & 5.7 \\
\hline Plant Physiology and Biochemistry (Paris) & v. 45 , fisrt page 389 , last page 394 & 0981-9428 & 1.847 & 5.7 \\
\hline Forest Ecology and Management & v. 184 , fisrt page 239 , last page 250 & 0378-1127 & 1.839 & 5.8 \\
\hline Forest Ecology and Management & v. 190 , fisrt page 323 , last page 333 & 0378-1127 & 1.839 & 5.8 \\
\hline Forest Ecology and Management & v. 191 , fisrt page 185 , last page 200 & 0378-1127 & 1.839 & 5.8 \\
\hline Forest Ecology and Management & v. 196 , fisrt page 199 , last page 212 & 0378-1127 & 1.839 & 5.8 \\
\hline Forest Ecology and Management & v. 208 , fisrt page 261 , last page 286 & 0378-1127 & 1.839 & 5.8 \\
\hline Forest Ecology and Management & v. 226, fisrt page 199, last page 309 & 0378-1127 & 1.839 & 5.8 \\
\hline Forest Ecology and Management & v. 226 , fisrt page 364 , last page 367 & 0378-1127 & 1.839 & 5.8 \\
\hline Forest Ecology and Management & v. 228 , fisrt page 61 , last page 68 & 0378-1127 & 1.839 & 5.8 \\
\hline Forest Ecology and Management & v. 229 , fisrt page 294 , last page 303 & $0378-1127$ & 1.839 & 5.8 \\
\hline Agriculture, Ecosystems \& Environment & v. 115 , fistt page 237 , last page 247 & 0167-8809 & 1.832 & 6.3 \\
\hline Fisheries Oceanography & v. 15 , fisrt page 37 , last page 43 & $1054-6006$ & 1.832 & 6.3 \\
\hline Journal of Zoological Systematics and & v. 42 , fisrt page 154 , last page 158 & 0947-5745 & & \\
\hline $\begin{array}{l}\text { Evolutionary Research } \\
\text { Journal of Zoological Systematics and }\end{array}$ & v. 42 , fistt page 306 , last page 312 & 0947-5745 & 1.826 & 4.8 \\
\hline Evolutionary Research & & & 1.826 & 4.8 \\
\hline $\begin{array}{l}\text { Journal of Zoological Systematics and } \\
\text { Evolutionary Research }\end{array}$ & v. 43 , fisrt page 199 , last page 213 & 0947-5745 & 1826 & 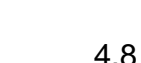 \\
\hline Journal of Zoological Systematics and & v. 43 , fisrt page 332 , last page 338 & 0947-5745 & & 4.0 \\
\hline Evolutionary Research & v 44 fist nage 212 last nage 211 & $0947-5745$ & 1.826 & 4.8 \\
\hline Evolutionary Research & & & 1.826 & 4.8 \\
\hline Palaeogeography, Palaeoclimatology, & v. 218 , fistt page 145 , last page 160 & 0031-0182 & & \\
\hline $\begin{array}{l}\text { Palaeoecology } \\
\text { Paleogeography Paleoclimatology }\end{array}$ & & & 1.822 & 7.3 \\
\hline Paleoecology & & & 1.822 & 7.3 \\
\hline Pattern Recognition & v. 37 , fisrt page 515 , last page 524 & 0031-3203 & 1.822 & 7.7 \\
\hline Archives of Microbiology & v. 184 , fistt page 194, last page 198 & $0302-8933$ & 1.82 & 10 \\
\hline Environmental and Experimental Botany & v. 14, fistt page 1, last page 14 & $0098-8472$ & 1.82 & 6.7 \\
\hline Environmental and Experimental Botany & v. 51 , fisrt page 45 , last page 56 & $0098-8472$ & 1.82 & 6.7 \\
\hline Environmental and Experimental Botany & v. 54, fisrt page 69 , last page 76 & 0098-8472 & 1.82 & 6.7 \\
\hline Environmental and Experimental Botany & v. 57 , fisrt page 100, last page 105 & 0098-8472 & 1.82 & 6.7 \\
\hline Environmental and Experimental Botany & v. 58 , fisrt page 123 , last page 129 & 0098-8472 & 1.82 & 6.7 \\
\hline Environmental and Experimental Botany & v. 58 , fisrt page 158 , last page 168 & 0098-8472 & 1.82 & 6.7 \\
\hline
\end{tabular}


Journal

Environmental and Experimental Botany

Mycorrhiza (Berlin)

Mycorrhiza (Berlin)

Chemico-Biological Interactions

Chemico-Biological Interactions

Journal of Foraminiferal Research

Parasitology (London)

Parasitology (London)

Carbohydrate Polymers

Carbohydrate Polymers

Clinical and Experimental Pharmacology and Physiology

Austral Ecology

Austral Ecology

Austral Ecology

Austral Ecology

Austral Ecology

Austral Ecology

Austral Ecology

Austral Ecology

Austral Ecology

Marine Biology (Berlin)

Marine Biology (Berlin)

Marine Biology (Berlin)

Marine Biology (Berlin)

Marine Biology (Berlin)

Marine Biology (Berlin)

Marine Biology (Berlin)

Journal of Comparative Physiology. A,

Sensory, Neural, and Behavioral

Physiology

Journal of Comparative Physiology. A,

Sensory, Neural, and Behavioral

Physiology

Journal of Comparative Physiology. A,

Sensory, Neural, and Behavioral

Physiology

Journal of Comparative Physiology. A,

Sensory, Neural, and Behavioral

Physiology

Planta Medica (Stuttgart)

Planta Medica (Stuttgart)

Planta Medica (Stuttgart)

Planta Medica (Stuttgart)

Planta Medica (Stuttgart)

Planta Medica (Stuttgart)

Planta Medica (Stuttgart)

Planta Medica (Stuttgart)

Planta Medica (Stuttgart)

Planta Medica (Stuttgart)

Planta Medica (Stuttgart)

Journal of Comparative Physiology. B,

Biochemical, Systemic, and Environmental

Physiology

Journal of Comparative Physiology. B,

Biochemical, Systemic, and Environmental

Physiology

Journal of Comparative Physiology. B,

Biochemical, Systemic, and Environmental Physiology

Biological Control

Biological Control

Estuarine, Coastal and Shelf Science
Paper info.

ISSN

v. 58, fist page 85, last page 92

v. 59 , fisrt page 11 , last page 20

v. 15 , fisrt page 47 , last page 53

v. 16 , fisrt page 183, last page 189

v. 151 , fisrt page 95 , last page 100

v. 159 , fisrt page 223 , last page 234

v. 1 , fist page 10 , last page 12

v. 128 , fisrt page 671 , last page 682

v. 131 , fisrt page 71 , last page 78

v. 60 , fisrt page 507 , last page 514

v. 66 , fisrt page 444 , last page 454

v. 33 , fisrt page 109 , last page 113

v. 29, fisrt page 342 , last page 349

v. 29 , fisrt page 437 , last page 445

v. 30 , fisrt page 243 , last page 249

v. 31 , fisrt page 343 , last page 348

v. 31 , fisrt page 449 , last page 457

v. 31 , fisrt page 776 , last page 782

v. 31 , fisrt page 869 , last page 881

v. 31 , fist page 95 , last page 98

v. n. 30 , fisrt page 58 , last page 73

v. 144 , fisrt page 119 , last page 126

v. 144 , fisrt page 999, last page 1010

v. 145 , fisrt page 603 , last page 613

v. 148 , fisrt page 1039, last page 1050

v. 148 , fisrt page 449 , last page 457

v. 148 , fisrt page 479 , last page 488

v. 149 , fisrt page 423 , last page 433

v. 190 , fisrt page 1 , last page 13

0098-8472 $1.82 \quad 6.7$

0098-8472

1.82

6.7

0940-6360

1.813

0940-6360

1.813

$0009-2797$

0009-2797

0096-1191

0031-1820

0031-1820

0144-8617

0144-8617

0305-1870

1442-9985

$1442-9985$

$1442-9985$

$1442-9985$

1442-9985

1442-9985

1442-9985

$1442-9985$

$1442-9985$

0025-3162

0025-3162

0025-3162

0025-3162

0025-3162

0025-3162

0025-3162

0340-7594

1.8

1.8

1.791

1.786

1.786

1.784

1.784

1.78

1.771

1.771

1.771

1.771

1.771

1.771

1.771

1.771

1.771

1.756

1.756

1.756

1.756

1.756

1.756

1.756

1.751

v. 190 , fisrt page 539 , last page 548

0340-7594

1.751

v. 190 , fisrt page 549 , last page 560

0340-7594

1.751

10

v. 192 , fisrt page 879 , last page 887

0340-7594

1.751

10

v. 70 , fisrt page 1 , last page 3

v. 70, fisrt page 1095, last page 1097

v. 70 , fisrt page 551 , last page 555

v. 70 , fisrt page 856 , last page 860

v. 71 , fist page 48 , last page 53

v. 71 , fisrt page 683 , last page 685

v. 71 , fisrt page 721 , last page 726

v. 72 , fisrt page 1 , last page 3

v. 72, fisrt page 295, last page 299

v. 72 , fisrt page 358 , last page 363

v. 72 , fisrt page 437 , last page 441

v. 174 , fisrt page 281 , last page 291

$\begin{array}{ll}1.746 & 8.7\end{array}$

$\begin{array}{ll}1.746 & 8.7\end{array}$

$\begin{array}{ll}1.746 & 8.7\end{array}$

$\begin{array}{ll}1.746 & 8.7\end{array}$

$\begin{array}{ll}1.746 & 8.7\end{array}$

$\begin{array}{ll}1.746 & 8.7\end{array}$

$\begin{array}{ll}1.746 & 8.7\end{array}$

$\begin{array}{ll}1.746 & 8.7\end{array}$

$\begin{array}{ll}1.746 & 8.7\end{array}$

$\begin{array}{ll}1.746 & 8.7\end{array}$

$\begin{array}{ll}1.746 & 8.7\end{array}$

1.74

8.7

v. 174 , fisrt page 91 , last page 96

0174-1578

1.74

8.7

v. 176 , fisrt page 125 , last page 138

0174-1578

v. 29 , fisrt page 1 , last page 4

v. 36, fisrt page 232, last page 237

v. 47 , fisrt page 613 , last page 627
1049-9644

1049-9644

1.735

1.733 


\begin{tabular}{|c|c|c|c|c|}
\hline Journal & Paper info. & ISSN & IF & Half-Life \\
\hline Estuarine, Coastal and Shelf Science & v. 65 , fistt page 1 , last page 18 & $0272-7714$ & 1.733 & 7.6 \\
\hline Estuarine, Coastal and Shelf Science & v. 66 , fisrt page 315 , last page 322 & $0272-7714$ & 1.733 & 7.6 \\
\hline Estuarine, Coastal and Shelf Science & v. 68 , fisrt page 600 , last page 608 & $0272-7714$ & 1.733 & 7.6 \\
\hline Estuarine, Coastal and Shelf Science & v. 69 , fisrt page 179 , last page 188 & $0272-7714$ & 1.733 & 7.6 \\
\hline Estuarine, Coastal and Shelf Science & v. 71 , fisrt page 1 , last page 10 & $0272-7714$ & 1.733 & 7.6 \\
\hline Estuarine, Coastal and Shelf Science & v. 71 , fisrt page 508 , last page 516 & $0272-7714$ & 1.733 & 7.6 \\
\hline Plant Cell Reports & v. 23 , fisrt page 251 , last page 255 & $0721-7714$ & 1.727 & 7.9 \\
\hline Plant Cell Reports & v. 24 , fisrt page 172 , last page 178 & $0721-7714$ & 1.727 & 7.9 \\
\hline Geomorphology (Amstrerdam) & v. 81, fisrt page 29 , last page 42 & $0169-555 X$ & 1.698 & 5.7 \\
\hline Cell Biochemistry and Biophysics & v. 44 , fisrt page 385 , last page 394 & $1085-9195$ & 1.693 & 4.2 \\
\hline Cell Biochemistry and Biophysics & v. 44 , fisrt page 412 , last page 423 & 1085-9195 & 1.693 & 4.2 \\
\hline Microscopy Research and Technique & v. 69 , fisrt page 885 , last page 890 & $1059-910 X$ & 1.68 & 6 \\
\hline Microscopy Research and Technique & v. 69 , fisrt page 913 , last page 918 & $1059-910 X$ & 1.68 & 6 \\
\hline American Journal of Human Biology & v. 16 , fisrt page 479 , last page 488 & $1042-0533$ & 1.669 & 5.1 \\
\hline Evolutionary Ecology & v. 18 , fisrt page 429 , last page 442 & $0269-7653$ & 1.667 & 9.2 \\
\hline Evolutionary Ecology, Research & v. 7, fisrt page 1051, last page 1065 & $0269-7653$ & 1.667 & 9.2 \\
\hline Archives of Oral Biology & v. 51 , fisrt page 15 , last page 22 & 0003-9969 & 1.655 & 10 \\
\hline Journal of Food Composition and Analysis & v. 19 , fisrt page 507 , last page 511 & $0889-1575$ & 1.655 & 5.4 \\
\hline Rivers Research and Applications & v. 21 , fisrt page 939 , last page 950 & $1535-1459$ & 1.645 & 3 \\
\hline Journal of Microbiology & v. 44 , fisrt page 591 , last page 599 & $1225-8873$ & 1.644 & 2 \\
\hline Aquaculture Nutrition & v. 11 , fisrt page 345 , last page 350 & $1353-5773$ & 1.642 & 5.2 \\
\hline Plant Science (Limerick) & v. 167, fistt page 949, last page 954 & 0168-9452 & 1.631 & 6.3 \\
\hline Plant Science (Limerick) & v. 168 , fistt page 1227 , last page 1233 & 0168-9452 & 1.631 & 6.3 \\
\hline Plant Science (Limerick) & v. 168, fistt page 723 , last page 729 & 0168-9452 & 1.631 & 6.3 \\
\hline Plant Science (Limerick) & v. 169, fistt page 629, last page 639 & 0168-9452 & 1.631 & 6.3 \\
\hline Plant Science (Limerick) & v. 169 , fistt page 819 , last page 832 & 0168-9452 & 1.631 & 6.3 \\
\hline Plant Science (Limerick) & v. 170 , fist page 624, last page 633 & 0168-9452 & 1.631 & 6.3 \\
\hline Plant Science (Limerick) & v. 171 , fistt page 91 , last page 98 & 0168-9452 & 1.631 & 6.3 \\
\hline Systematic Botany & v. 29, fisrt page 50 , last page 63 & 0363-6445 & 1.629 & 7 \\
\hline Systematic Botany & v. 30 , fisrt page 183, last page 194 & 0363-6445 & 1.629 & 7 \\
\hline Systematic Botany & v. 30 , fisrt page 357 , last page 365 & 0363-6445 & 1.629 & 7 \\
\hline Systematic Botany & v. 31 , fisrt page 138 , last page 150 & $0363-6445$ & 1.629 & 7 \\
\hline Systematic Botany & v. 31 , fisrt page 298 , last page 301 & $0363-6445$ & 1.629 & 7 \\
\hline Systematic Botany & v. 31 , fisrt page 320 , last page 326 & $0363-6445$ & 1.629 & 7 \\
\hline Systematic Botany & v. 31 , fisrt page 493 , last page 496 & $0363-6445$ & 1.629 & 7 \\
\hline Systematic Botany & v. 31 , fisrt page 506 , last page 511 & $0363-6445$ & 1.629 & 7 \\
\hline Systematic Botany & v. 31 , fisrt page 822 , last page 825 & $0363-6445$ & 1.629 & 7 \\
\hline Journal od Ethnopharmacology & v. 108 , fisrt page 103 , last page 108 & $0378-8741$ & 1.625 & 6.1 \\
\hline Journal of Ethnopharmacology & v. 100 , fisrt page 254 , last page 259 & $0378-8741$ & 1.625 & 6.1 \\
\hline Journal of Ethnopharmacology & v. 101, fisrt page 61, last page 67 & $0378-8741$ & 1.625 & 6.1 \\
\hline Journal of Ethnopharmacology & v. 103, fistt page 53 , last page 58 & $0378-8741$ & 1.625 & 6.1 \\
\hline Journal of Ethnopharmacology & v. 104 , fisrt page 225 , last page 233 & $0378-8741$ & 1.625 & 6.1 \\
\hline Journal of Ethnopharmacology & v. 105 , fisrt page 173 , last page 186 & $0378-8741$ & 1.625 & 6.1 \\
\hline Journal of Ethnopharmacology & v. 107 , fisrt page 291 , last page 296 & $0378-8741$ & 1.625 & 6.1 \\
\hline Journal of Ethnopharmacology & v. 23 , fisrt page 150 , last page 182 & $0378-8741$ & 1.625 & 6.1 \\
\hline Journal of Ethnopharmacology & v. 92 , fisrt page 269 , last page 273 & 0378-8741 & 1.625 & 6.1 \\
\hline Journal of Ethnopharmacology & v. 95 , fisrt page 345 , last page 351 & 0378-8741 & 1.625 & 6.1 \\
\hline Journal of Ethnopharmacology & v. 97 , fisrt page 183 , last page 189 & $0378-8741$ & 1.625 & 6.1 \\
\hline Journal of Ethnopharmacology & v. 97 , fisrt page 305 , last page 311 & $0378-8741$ & 1.625 & 6.1 \\
\hline Journal of Ethnopharmacology & v. 99, fisrt page 21 , last page 30 & $0378-8741$ & 1.625 & 6.1 \\
\hline Journal of Ethnopharmacology & v. 99 , fisrt page 37, last page 41 & $0378-8741$ & 1.625 & 6.1 \\
\hline International Journal of Plant Sciences & v. 166 , fist page 493 , last page 506 & $1058-5893$ & 1.622 & 5.8 \\
\hline International Biodeterioration and & v. 55, fisrt page 187, last page 193 & $0964-8305$ & & \\
\hline & & & 1.619 & 6.5 \\
\hline Soil \& Tillage Research & v. 90 , fisrt page 16 , last page 28 & 0167-1987 & 1.619 & 7.4 \\
\hline Journal of Plankton Research & v. 26 , fisrt page 1277 , last page 1287 & $0142-7873$ & 1.617 & 8.6 \\
\hline Journal of Plankton Research & v. 26 , fisrt page 859 , last page 874 & $0142-7873$ & 1.617 & 8.6 \\
\hline Journal of Plankton Research & v. 26 , fisrt page 983 , last page 992 & $0142-7873$ & 1.617 & 8.6 \\
\hline Journal of Plankton Research & v. 27 , fisrt page 683 , last page 694 & $0142-7873$ & 1.617 & 8.6 \\
\hline Chemistry \& Biodiversity & v. 1, fisrt page 1 , last page 9 & $1612-1872$ & 1.616 & 2.2 \\
\hline
\end{tabular}




\begin{tabular}{l} 
Journal \\
\hline Restoration Ecology \\
Restoration Ecology \\
Magnetic Resonance in Chemistry \\
Magnetic Resonance in Chemistry \\
Quaternary International \\
European Journal of Applied Physiology \\
Ibis (London) \\
Letters in Applied Microbiology \\
Letters in Applied Microbiology \\
Letters in Applied Microbiology \\
Environmental Toxicology \\
Environmental Toxicology \\
Environmental Toxicology \\
Journal of Physics. A, Mathematical and \\
General \\
Mycologia \\
Mycologia \\
Mycologia \\
Antarctic Science \\
Antarctic Science \\
Oncology Reports \\
Estuaries \\
Estuaries \\
Estuaries \\
Microchemical Journal \\
Invertebrate Biology \\
Invertebrate Biology \\
Comparative Biochemistry and Physiology. \\
A
\end{tabular}

Comparative Biochemistry and Physiology. A, Molecular \& Integrative Physiology

Comparative Biochemistry and Physiology. A, Molecular \& Integrative Physiology

Comparative Biochemistry and Physiology.

A, Molecular \& Integrative Physiology

Comparative Biochemistry and Physiology.

A, Molecular \& Integrative Physiology

Comparative Biochemistry and Physiology.

A, Molecular \& Integrative Physiology

Journal of Morphology

Journal of Morphology

Journal of Morphology

Journal of Comparative Psychology

Helvetica Chimica Acta

Helvetica Chimica Acta

Journal of Mammalogy

Journal of Mammalogy

Journal of Mammalogy

Journal of Mammalogy

Journal of Mammalogy

Journal of Mammalogy

Journal of Mammalogy

Journal of Mammalogy

Review of Scientific Instruments

Population Ecology

Population Ecology

Journal of Pharmacy and Pharmacology

Journal of Pharmacy and Pharmacology

Journal of Pharmacy and Pharmacology

Comparative Biochemistry and Physiology.

B, Biochemistry \& Molecular Biology

Comparative Biochemistry and Physiology.

B, Biochemistry \& Molecular Biology

Journal of Environmental Monitoring v. 14, fisrt page 504, last page 512

v. 14 , fisrt page 87 , last page 94

v. 43 , fisrt page 180 , last page 183

v. 43 , fisrt page 864 , last page 866

v. 114 , fisrt page 87 , last page 99

v. 18 , fisrt page 287 , last page 294

v. 146 , fisrt page 438 , last page 453

v. 38 , fisrt page 93 , last page 98

v. 39 , fist page 34 , last page 40

v. 40 , fisrt page 19 , last page 23

v. 19 , fisrt page 578 , last page 584

v. 20 , fisrt page 126 , last page 130

v. 21 , fisrt page 95, last page 103

v. 34 , fisrt page 732 , last page 736

v. 96 , fisrt page 151 , last page 154

v. 97 , fisrt page 304 , last page 311

v. 98 , fisrt page 821 , last page 827

v. 16 , fisrt page 129 , last page 131

v. 18 , fisrt page 163 , last page 170

v. 13 , fisrt page 943 , last page 947

v. 27 , fisrt page 905 , last page 914

v. 28 , fisrt page 675 , last page 685

v. 29 , fisrt page 277 , last page 285

v. 82 , fisrt page 196, last page 200

v. 1 , fisrt page 23 , last page 42

v. 124 , fisrt page 79 , last page 87

v. 125 , fisrt page 212 , last page 221

v. 137 , fisrt page 383 , last page 390

v. 142, fisrt page 495, last page 502

v. 144 , fisrt page 33 , last page 40

v. 146 , fisrt page 486 , last page 498

v. 4 , fisrt page 1 , last page 6

v. 260 , fisrt page 234 , last page 246

v. 267 , fisrt page 187 , last page 197

v. 42, fisrt page 163, last page 172

v. 119 , fisrt page 343 , last page 351

v. 88, fisrt page 1102, last page 1109

v. 88 , fist page 3225 , last page 3231

v. 85 , fist page 1201, last page 1206

v. 85 , fisrt page 148 , last page 161

v. 85 , fist page 82 , last page 95

v. 86 , fisrt page 287 , last page 293

v. 86 , fisrt page 403 , last page 414

v. 86 , fisrt page 873 , last page 879

v. 86 , fisrt page 902 , last page 908

v. 87 , fisrt page 757 , last page 765

v. 75 , fist page 2213 , last page 2215

v. 47 , fisrt page 213 , last page 219

v. 48 , fisrt page 151 , last page 158

v. 56 , fisrt page 1 , last page 6

v. 56 , fisrt page 667 , last page 681

v. 58 , fisrt page 235 , last page 241

v. 11 , fisrt page 1 , last page 12

v. 145 , fisrt page 101, last page 107

v. 7, fisrt page 493, last page 499
ISSN

1061-2971

1061-2971

IF

Half-Life

0749-1581

.612

1.612

0749-1581

1.61

1040-6182

1.61

1439-6319

1.607

1.601

0019-1019 1.595

0266-8254

1.593

0266-8254

0266-8254

1.593

1.593

1520-4081

1520-4081

1.582

1.582

1520-4081

0305-4470

0027-5514

1.582

1.577

1.574

0027-5514

0027-5514

0954-1020

1.574

1.574

1.573

0954-1020

1021-335X

0160-8347

0160-8347

0160-8347

0026-265X

1077-8306

1077-8306

1077-8306

1095-6433

1.573

1.567

1.563

1.563

1.563

1.558

1.556

1.556

1.556

1.553

9.2

1095-6433

1.553

9.2

1095-6433

1095-6433

1.553

9.2

1095-6433

0362-2525

0362-2525

0362-2525

0735-7036

0018-019X

0018-019X

0022-2372

0022-2372

0022-2372

0022-2372

0022-2372

0022-2372

0022-2372

0022-2372

0034-6748

1438-3896

1438-3896

0022-3573

0022-3573

0022-3573

1096-4959

1.553

9.2

1.553

9.2

1.553

1.553

1.553

1.552

1.55

1.55

1.549

1.549

1.549

1.549

1.549

1.549

1.549

1.549

1.541

1.534

1.534

1.533

1.533

1.533

1.532

9.6

1096-4959

1464-0325 
Journal

Biological \& Pharmaceutical Bulletin

Biological \& Pharmaceutical Bulletin

Biological \& Pharmaceutical Bulletin

Biological \& Pharmaceutical Bulletin

Journal of Plant Physiology

Journal of Plant Physiology

Journal of Plant Physiology

Journal of Plant Physiology

Animal Genetics

Animal Genetics

Chemotherapy (Basel)

Folia Parasitologica

Structural Chemistry

Polar Biology

Polar Biology

Polar Biology

Polar Biology

Polar Biology

Plant and Soil

Plant and Soil

Plant and Soil

Genetica (The Hague)

Genetica (The Hague)

Genetica (The Hague)

Genetica (The Hague)

Genetica (The Hague)

Genetica (The Hague)

Genetica (The Hague)

Genetica (The Hague)

Genetica (The Hague)

Genetica (The Hague)

Genetica (The Hague)

Genetica (The Hague)

Genetica (The Hague)

Genetica (The Hague)

Genetica (The Hague)

Genetica (The Hague)

Genetica (The Hague)

Genetica (The Hague)

Genetica (The Hague)

Genetica (The Hague)

Genetica (The Hague)

Genetica (The Hague)

Genetica (The Hague)

Genetica (The Hague)

Genetica (The Hague)

Inflammation Research

Malacologia

Malacologia

Visual Neuroscience

Insectes Sociaux

Insectes Sociaux

Insectes Sociaux

Insectes Sociaux

Insectes Sociaux

Insectes Sociaux

Insectes Sociaux

Insectes Sociaux

Insectes Sociaux
Paper info.

v. 27 , fist page 52 , last page 55

v. 27 , fisrt page 821 , last page 824

v. 28, fist page 1010, last page 1015

v. 29 , fisrt page 135 , last page 140

v. 29, fist page 2307, last page 2309

v. 161 , fisrt page 1385, last page 1387

v. 161 , fisrt page 769 , last page 776

v. 161 , fisrt page 929 , last page 935

v. 163 , fisrt page 1126, last page 1134

v. 35 , fisrt page 278 , last page 284

v. 6 , fisrt page 462, last page 504

v. 52 , fisrt page 91 , last page 94

v. 53 , fisrt page 107 , last page 116

v. 6 , fisrt page 9 , last page 18

v. 1 , fist page 10 , last page 20

v. 27 , fist page 75 , last page 82

v. 28 , fisrt page 372 , last page 380

v. 29 , fisrt page 389 , last page 398

v. 29 , fisrt page 552 , last page 561

v. 258 , fisrt page 293 , last page 306

v. 270 , fisrt page 169 , last page 178

v. 286 , fisrt page 323 , last page 337

v. 1 , fisrt page 227 , last page 235

v. 121 , fisrt page 125 , last page 132

v. 121 , fisrt page 241 , last page 249

v. 121 , fist page 89 , last page 94

v. 123 , fisrt page 271 , last page 283

v. 125 , fisrt page 125 , last page 132

v. 125 , fisrt page 311 , last page 323

v. 126 , fisrt page 1 , last page 3

v. 126, fisrt page 1 , last page 9

v. 126 , fisrt page 101 , last page 110

v. 126 , fisrt page 141 , last page 151

v. 126 , fist page 15 , last page 32

v. 126 , fisrt page 215 , last page 225

v. 126 , fisrt page 3 , last page 14

v. 126 , fist page 57 , last page 75

v. 126 , fisrt page 77 , last page 88

v. 127 , fisrt page 133 , last page 141

v. 127 , fisrt page 243 , last page 252

v. 127 , fist page 35 , last page 44

v. 128, fisrt page 1, last page 9

v. 128 , fisrt page 287 , last page 295

v. 128 , fisrt page 333 , last page 346

v. 128 , fisrt page 449 , last page 453

v. n. 1 , fisrt page 127 , last page 136

v. n. 1 , fisrt page 205 , last page 210

v. 55 , fisrt page 129 , last page 135

v. 46 , fisrt page 1 , last page 22

v. 46 , fisrt page 355 , last page 380

v. 23 , fisrt page 1 , last page 8

v. 51 , fisrt page 17 , last page 23

v. 51, fisrt page 221, last page 225

v. 51 , fist page 37 , last page 42

v. 51 , fisrt page 400 , last page 409

v. 52 , fisrt page 194, last page 200

v. 52 , fisrt page 327 , last page 332

v. 52 , fisrt page 333 , last page 338

v. 52 , fisrt page 352 , last page 358

v. 53 , fisrt page 265 , last page 268
ISSN

0918-6158

IF

1.522

Half-Life

0918-6158

1.522

0918-6158 1.522

0918-6158 1.522

0918-6158 1.522

0176-1617 1.521

0176-1617 1.521

0176-1617 1.521

0176-1617 1.521

0268-9146

0268-9146

0009-3157

0015-5683

1040-0400

0722-4060

0722-4060

0722-4060

0722-4060

0722-4060

0032-079X

0032-079X

0032-079X

0016-6707

0016-6707

0016-6707

0016-6707

0016-6707

0016-6707

0016-6707

0016-6707

0016-6707

0016-6707

0016-6707

0016-6707

0016-6707

0016-6707

0016-6707

0016-6707

0016-6707

0016-6707

0016-6707

0016-6707

0016-6707

0016-6707

0016-6707

0016-6707

0016-6707

1023-3830

0076-2997

0076-2997

0952-5238

1.52

1.52

1.511

1.511

1.51

1.502

1.502

1.502

1.502

1.502

1.495

1.495

1.495

1.492

1.492

1.492

1.492

1.492

1.492

1.492

1.492

1.492

1.492

1.492

1.492

1.492

1.492

1.492

1.492

1.492

1.492

1.492

1.492

1.492

1.492

1.492

1.492

1.492

1.485

1.484

1.484

1.484

1.481

1.481

1.481

1.481

1.481

1.481

1.481

1.481

$\begin{array}{ll}0020-1812 & 1.481 \\ 0020-1812 & 1.481\end{array}$

0020-1812

.

4.7

4.7

4.7

4.7

4.7

9

9

9

9

6.8

6.8

7.2

8.3

5

7.7

7.7

7.7

7.7

7.7

9.3

9.3

9.3

6.6

6.6

6.6

6.6

6.6

6.6

6.6

6.6

6.6

6.6

6.6

6.6

6.6

6.6

6.6

6.6

6.6

6.6

6.6

6.6

6.6

6.6

6.6

6.6

6.6

5.9

10

10

9.3

8.9

8.9

8.9

8.9

8.9

8.9

8.9

8.9

8.9 


\begin{tabular}{|c|c|c|c|c|}
\hline Journal & Paper info. & ISSN & IF & Half-Life \\
\hline Insectes Sociaux & v. 53 , fisrt page 472 , last page 479 & 0020-1812 & 1.481 & 8.9 \\
\hline Ecology of Freshwater Fish & v. 15 , fisrt page 284 , last page 290 & 0906-6691 & 1.479 & 6.1 \\
\hline Ecology of Freshwater Fish & v. 32, fisrt page 87 , last page 94 & $0906-6691$ & 1.479 & 6.1 \\
\hline Behavioural Processes & v. 66 , fisrt page 43 , last page 51 & 0376-6357 & 1.478 & 6.9 \\
\hline Behavioural Processes & v. 67 , fisrt page 471 , last page 476 & $0376-6357$ & 1.478 & 6.9 \\
\hline Behavioural Processes & v. 70 , fisrt page 149 , last page 155 & $0376-6357$ & 1.478 & 6.9 \\
\hline Behavioural Processes & v. 70, fisrt page 32 , last page 40 & $0376-6357$ & 1.478 & 6.9 \\
\hline Behavioural Processes & v. 73 , fisrt page 228 , last page 230 & 0376-6357 & 1.478 & 6.9 \\
\hline Archives of Insect Biochemistry and & v. 56, fisrt page 84, last page 96 & $0739-4462$ & & \\
\hline $\begin{array}{l}\text { Physiology } \\
\text { Archives of Insect Biochemistry and }\end{array}$ & v. 59 , fisrt page 211 , last page 218 & 0739-4462 & 1.474 & 8.3 \\
\hline $\begin{array}{l}\text { Physiology } \\
\text { Phect Diochermitry and }\end{array}$ & V. J9, IISt page 211 , last page $\angle 10$ & 0739-4402 & 1.474 & 8.3 \\
\hline $\begin{array}{l}\text { Archives of Insect Biochemistry and } \\
\text { Physiology }\end{array}$ & v. 63 , fisrt page 57 , last page 72 & $0739-4462$ & 1.474 & 8.3 \\
\hline Agricultural and Forest Entomology & v. 8 , fisrt page 17 , last page 23 & 1461-9555 & 1.473 & 4.4 \\
\hline ICES Journal of Marine Science & v. 61 , fisrt page 798 , last page 811 & $1054-3139$ & 1.469 & 6.5 \\
\hline ICES Journal of Marine Science & v. 62 , fisrt page 1095, last page 1103 & $1054-3139$ & 1.469 & 6.5 \\
\hline ICES Journal of Marine Science & v. 62 , fisrt page 201, last page 213 & 1054-3139 & 1.469 & 6.5 \\
\hline ICES Journal of Marine Science & v. 63 , fisrt page 883 , last page 896 & $1054-3139$ & 1.469 & 6.5 \\
\hline Journal of Biochemistry and Molecular & v. 39 , fisrt page 216 , last page 222 & $1225-8687$ & 1465 & 3.2 \\
\hline Trees (Berlin) & v. 19 , fisrt page 280 , last page 282 & 0931-1890 & 1.461 & $\begin{array}{l}0.4 \\
6.6\end{array}$ \\
\hline Trees (Berlin) & v. 19 , fisrt page 296, last page 304 & 0931-1890 & 1.461 & 6.6 \\
\hline Trees (Berlin) & v. 19 , fisrt page 326 , last page 335 & 0931-1890 & 1.461 & 6.6 \\
\hline Trees (Berlin) & v. 19 , fisrt page 422 , last page 430 & 0931-1890 & 1.461 & 6.6 \\
\hline Trees (Berlin) & v. 19 , fisrt page 493 , last page 496 & 0931-1890 & 1.461 & 6.6 \\
\hline Trees (Berlin) & v. 19 , fisrt page 497 , last page 509 & 0931-1890 & 1.461 & 6.6 \\
\hline Trees (Berlin) & v. 19 , fisrt page 510 , last page 522 & 0931-1890 & 1.461 & 6.6 \\
\hline Trees (Berlin) & v. 19 , fisrt page 523 , last page 530 & 0931-1890 & 1.461 & 6.6 \\
\hline Trees (Berlin) & v. 20, fisrt page 286, last page 291 & $0931-1890$ & 1.461 & 6.6 \\
\hline Trees (Berlin) & v. 20 , fisrt page 669 , last page 678 & $0931-1890$ & 1.461 & 6.6 \\
\hline Journal of Marine Systems & v. 60 , fisrt page 268 , last page 284 & $0924-7963$ & 1.447 & 6.1 \\
\hline Ambio (Oslo) & v. 33, fisrt page 68, last page 77 & 0044-7447 & 1.433 & 7.9 \\
\hline American Journal of Primatology & v. 62 , fisrt page 171 , last page 187 & $0275-2565$ & 1.429 & 9.4 \\
\hline American Journal of Primatology & v. 64 , fisrt page 337 , last page 343 & $0275-2565$ & 1.429 & 9.4 \\
\hline American Journal of Primatology & v. 65 , fisrt page 141 , last page 147 & $0275-2565$ & 1.429 & 9.4 \\
\hline American Journal of Primatology & v. 67, fisrt page 69 , last page 81 & $0275-2565$ & 1.429 & 9.4 \\
\hline American Journal of Primatology & v. 68 , fisrt page 1120 , last page 1126 & $0275-2565$ & 1.429 & 9.4 \\
\hline American Journal of Primatology & v. 68, fisrt page 209 , last page 215 & $0275-2565$ & 1.429 & 9.4 \\
\hline American Journal of Primatology & v. 68 , fisrt page 825 , last page 831 & $0275-2565$ & 1.429 & 9.4 \\
\hline Conservation Genetics & v. 1 , fisrt page 12, last page 19 & $1566-0621$ & 1.429 & 3.9 \\
\hline Conservation Genetics & v. 5 , fisrt page 155, last page 157 & $1566-0621$ & 1.429 & 3.9 \\
\hline Biodiversity and Conservation & v. 13 , fisrt page 1419 , last page 1425 & $0960-3115$ & 1.423 & 5.9 \\
\hline Biodiversity and Conservation & v. 13 , fisrt page 1611 , last page 1632 & $0960-3115$ & 1.423 & 5.9 \\
\hline Biodiversity and Conservation & v. 13, fisrt page 2055, last page 2066 & $0960-3115$ & 1.423 & 5.9 \\
\hline Biodiversity and Conservation & v. 13 , fisrt page 2185 , last page 2207 & 0960-3115 & 1.423 & 5.9 \\
\hline Biodiversity and Conservation & v. 13 , fisrt page 2295 , last page 2318 & 0960-3115 & 1.423 & 5.9 \\
\hline Biodiversity and Conservation & v. 13 , fisrt page 2567 , last page 2587 & 0960-3115 & 1.423 & 5.9 \\
\hline Biodiversity and Conservation & v. 13, fisrt page 2679, last page 2694 & 0960-3115 & 1.423 & 5.9 \\
\hline Biodiversity and Conservation & v. 14, fisrt page 1047, last page 1057 & 0960-3115 & 1.423 & 5.9 \\
\hline Biodiversity and Conservation & v. 14 , fisrt page 1225 , last page 1240 & 0960-3115 & 1.423 & 5.9 \\
\hline Biodiversity and Conservation & v. 14, fisrt page 1777, last page 1798 & 0960-3115 & 1.423 & 5.9 \\
\hline Biodiversity and Conservation & v. 14, fisrt page 1929, last page 1945 & 0960-3115 & 1.423 & 5.9 \\
\hline Biodiversity and Conservation & v. 14, fisrt page 2883, last page 2899 & 0960-3115 & 1.423 & 5.9 \\
\hline Biodiversity and Conservation & v. 15 , fisrt page 2737 , last page 2746 & 0960-3115 & 1.423 & 5.9 \\
\hline Biodiversity and Conservation & v. 15 , fisrt page 2747 , last page 2764 & 0960-3115 & 1.423 & 5.9 \\
\hline Biodiversity and Conservation & v. 15, fisrt page 2905, last page 2923 & 0960-3115 & 1.423 & 5.9 \\
\hline Biodiversity and Conservation & v. 15 , fisrt page 4197 , last page 4210 & 0960-3115 & 1.423 & 5.9 \\
\hline Biodiversity and Conservation & v. 15 , fisrt page 4357 , last page 4373 & $0960-3115$ & 1.423 & 5.9 \\
\hline Biodiversity and Conservation & v. 15 , fisrt page 587 , last page 612 & 0960-3115 & 1.423 & 5.9 \\
\hline Biodiversity and Conservation & v. 16, fisrt page 291, last page 312 & 0960-3115 & 1.423 & 5.9 \\
\hline Biodiversity and Conservation & v. online, fisrt page 3429 , last page 3439 & 0960-3115 & 1.423 & 5.9 \\
\hline
\end{tabular}


Journal and Toxicology

Archives of Environmental Contamination and Toxicology

Journal of Vertebrate Paleontology

Journal of Vertebrate Paleontology

Journal of Vertebrate Paleontology

Journal of Vertebrate Paleontology

Journal of Vertebrate Paleontology

Journal of Industrial Microbiology and

Biotechnology

Journal of Zoology

Journal of Zoology

Journal of Zoology

Journal of Zoology

Journal of Zoology

Journal of Zoology

Journal of Zoology

Journal of Zoology

Journal of Zoology

Journal of Zoology

Journal of Zoology

Journal of Zoology

Fishery Bulletin (Washington)

Fishery Bulletin (Washington)

Phytomedicine (Stuttgart)

Phytomedicine (Stuttgart)

Phytomedicine (Stuttgart)

Phytomedicine (Stuttgart)

Veterinary Dermatology

Veterinary Dermatology

Forensic Science International

Canadian Journal of Zoology

Canadian Journal of Zoology

Canadian Journal of Zoology

Biotropica (Lawrence)

Biotropica (Lawrence, KS)

Biotropica (Lawrence, KS)

Biotropica (Lawrence, KS)

Biotropica (Lawrence, KS)

Biotropica (Lawrence, KS)

Biotropica (Lawrence, KS)

Biotropica (Lawrence, KS)

Biotropica (Lawrence, KS)

Biotropica (Lawrence, KS)

Biotropica (Lawrence, KS)

Biotropica (Lawrence, KS)

Biotropica (Lawrence, KS)

Biotropica (Lawrence, KS)

Biotropica (Lawrence, KS)

Biotropica (Lawrence, KS)

Biotropica (Lawrence, KS)

Entomologia Experimentalis et Applicata

Entomologia Experimentalis et Applicata

Entomologia Experimentalis et Applicata

Plant Ecology

Plant Ecology

Plant Ecology

Plant Ecology

Plant Ecology (Dordrecht)

Canadian Journal of Physiology and

Pharmacology
Paper info.

ISSN

v. 46, fisrt page 463, last page 469

v. 48 , fisrt page 381 , last page 390

0090-4341

IF

Half-Life

v. 2004, 109A, 110A, Inglês, ,

v. 2004, 113A, 113A, Inglês, ,

v. 2004, 39A, 39A, Inglês, ,

v. 2004, 78A, 78A, Inglês, ,

v. 24 , fisrt page 971 , last page 973

v. 32 , fisrt page 481 , last page 486

v. 265 , fisrt page 1 , last page 8

v. 265 , fisrt page 359 , last page 364

v. 266 , fisrt page 355 , last page 364

v. 266 , fisrt page 384 , last page 394

v. 266 , fisrt page 385 , last page 394

v. 266 , fisrt page 55 , last page 63

v. 267 , fisrt page 1 , last page 11

v. 268 , fisrt page 335 , last page 340

v. 269 , fisrt page 221 , last page 224

v. 270 , fisrt page 692 , last page 698

v. 271 , fist page 1 , last page 10

v. 271 , fist page 27 , last page 37

v. 102 , fisrt page 156, last page 167

v. 102, fisrt page 581, last page 592

v. 11 , fist page 114 , last page 120

v. 12 , fisrt page 424 , last page 432

v. 12 , fisrt page 72 , last page 77

v. 12 , fisrt page 78 , last page 87

v. 15 , fisrt page 269 , last page 277

v. 17 , fisrt page 453 , last page 455

v. 155 , fisrt page 61 , last page 64

v. 82 , fist page 1871 , last page 1877

v. 84 , fisrt page 136 , last page 140

v. 84 , fist page 1763, last page 1774

v. 38, fisrt page 711 , last page 717

v. 20 , fisrt page 471 , last page 474

v. 35 , fisrt page 419 , last page 421

v. 36 , fisrt page 371 , last page 376

v. 36 , fisrt page 417 , last page 426

v. 36 , fisrt page 641 , last page 646

v. 36 , fisrt page 74 , last page 84

v. 37 , fisrt page 166 , last page 179

v. 37 , fisrt page 381 , last page 388

v. 37 , fisrt page 415 , last page 419

v. 38 , fisrt page 229 , last page 234

v. 38 , fisrt page 27 , last page 34

v. 38 , fisrt page 383 , last page 389

v. 38 , fisrt page 514 , last page 521

v. 38 , fisrt page 674 , last page 678

v. 38 , fisrt page 786 , last page 788

v. 44 , fisrt page 261 , last page 270

v. 118 , fisrt page 185, last page 192

v. 119 , fist page 53 , last page 60

v. 121 , fisrt page 23 , last page 29

v. 174 , fisrt page 339 , last page 348

v. 174 , fisrt page 59 , last page 70

v. 175 , fisrt page 37 , last page 46

v. 180 , fisrt page 161 , last page 173

v. 180 , fisrt page 87 , last page 104

v. 82 , fisrt page 319 , last page 325
0090-4341

$1.419 \quad 8.1$

0272-4634

1.419

8.1

$0272-4634$

$0272-4634$

$0272-4634$

$0272-4634$

1367-5435

0952-8369

0952-8369

0952-8369

0952-8369

0952-8369

0952-8369

0952-8369

1.418

1.418

1.418

1.418

1.418

1.416

1.413

1.413

1.413

1.413

1.413

1.413

1.413

0952-8369 1.413

0952-8369

0952-8369

0952-8369

0952-8369

0090-0656

0090-0656

0944-7113

0944-7113

0944-7113

0944-7113

0959-4493

0959-4493

0379-0738

0008-4301

0008-4301

0008-4301

0006-3606

0006-3606

0006-3606

0006-3606

0006-3606

0006-3606

0006-3606

0006-3606

0006-3606

0006-3606

0006-3606

0006-3606

0006-3606

0006-3606

0006-3606

0006-3606

0006-3606

0013-8703

0013-8703

0013-8703

1573-5052

1573-5052

1573-5052

1573-5052

1385-0237

0008-4212

1.413

1.413

1.413

1.403

1.403

1.403

1.403

1.403

1.403

1.398

1.398

1.397

1.393

1.393

1.393

1.391

1.391

1.391

1.391

1.391

1.391

1.391

1.391

1.391

1.391

1.391

1.391

1.391

1.391

1.391

1.391

1.391

1.391

1.391

1.391

1.383

1.383

1.383

1.383

1.383

1.38

10 
Journal

Fisheries Management and Ecology

Fisheries Management and Ecology

Fisheries Management and Ecology

Cell Biology International

Cell Biology International

Cell Biology International

Tropical Studies in Oceanography

Physiological Entomology

Journal of Experimental Zoology

Environmental Entomology

Aquatic Botany

Aquatic Botany

Aquatic Botany

Aquatic Botany

Aquatic Botany

Aquatic Botany

Aquatic Botany

Hydrology and Earth System Sciences

Protoplasma

Protoplasma

International Journal of Primatology

International Journal of Primatology

Annals of the Missouri Botanical Garden

Annals of the Missouri Botanical Garden

Annals of the Missouri Botanical Garden

Annals of the Missouri Botanical Garden

International Journal of Biological

Macromolecules,

Acta Oecologica (Montrouge)

Acta Oecologica (Montrouge)

Acta Oecologica (Montrouge)

Acta Oecologica (Montrouge)

Acta Oecologica (Montrouge)

Annales Zoologici Fennici

Journal of Parasitology

Journal of Parasitology

Journal of Parasitology

European Journal of Phycology

Animal Feed Science and Technology

Annals of Forest Science

Annals of the Entomological Society of America

Annals of the Entomological Society of America

Annals of the Entomological Society of America

Annals of the Entomological Society of America

Annals of the Entomological Society of America

Annals of the Entomological Society of America

Invertebrate Systematics

Invertebrate Systematics

Anatomy and Embryology

Journal of Tropical Ecology

Journal of Tropical Ecology

Journal of Tropical Ecology

Journal of Tropical Ecology

Journal of Tropical Ecology

Journal of Tropical Ecology

Journal of Tropical Ecology
Paper info.

ISSN

v. 11, fisrt page 135, last page 137

v. 11 , fist page 45 , last page 50

v. 11 , fisrt page 71 , last page 79

v. 12 , fisrt page 63 , last page 67

v. 28 , fisrt page 335 , last page 344

v. 29 , fisrt page 778 , last page 782

v. 30 , fisrt page 485 , last page 494

v. 51 , fisrt page 149 , last page 172

v. 31 , fisrt page 371 , last page 381

v. $301 \mathrm{~A}$, fisrt page 240 , last page 248

v. 33, fisrt page 1217, last page 1222

v. 4, fisrt page 295, last page 305

v. 79, fisrt page 325 , last page 332

v. 80 , fisrt page 241 , last page 251

v. 81 , fisrt page 343 , last page 352

v. 82 , fisrt page 193, last page 209

v. 84 , fisrt page 183 , last page 190

v. 85 , fisrt page 16 , last page 20

v. 9 , fisrt page 570 , last page 585

v. 224 , fist page 33 , last page 40

v. 225 , fisrt page 123 , last page 128

v. 26 , fisrt page 1321 , last page 1344

v. 27, fist page 1097, last page 1101

v. 91 , fisrt page 303 , last page 319

v. 91 , fisrt page 474 , last page 485

v. 91 , fisrt page 973 , last page 984

v. 93, fisrt page 465, last page 516

v. 36 , fist page 90 , last page 97

v. 100 , fisrt page 1 , last page 10

v. 28 , fisrt page 289 , last page 298

v. 29, fisrt page 311 , last page 315

v. 30 , fisrt page 1 , last page 8

v. 61 , fisrt page 749 , last page 755

v. 42 , fisrt page 513 , last page 521

v. 91 , fist page 1000, last page 1007

v. 91, fisrt page 1025, last page 1027

v. 92 , fisrt page 459 , last page 463

v. 39 , fisrt page 17 , last page 32

v. 120 , fisrt page 151 , last page 157

v. 61 , fisrt page 87 , last page 95

v. 97 , fisrt page 729 , last page 731

v. 97 , fisrt page 865 , last page 871

v. 98 , fisrt page 515 , last page 526

v. 99 , fisrt page 409 , last page 432

v. 99 , fisrt page 648, last page 655

v. 99 , fisrt page 831 , last page 836

v. 19 , fisrt page 557 , last page 576

v. 20 , fisrt page 43 , last page 58

v. 211 , fisrt page 423 , last page 434

v. 20 , fisrt page 471 , last page 474

v. 20 , fisrt page 701 , last page 704

v. 21 , fisrt page 121 , last page 125

v. 21 , fisrt page 189 , last page 194

v. 21 , fisrt page 397 , last page 406

v. 21 , fisrt page 529 , last page 540

v. 22 , fisrt page 1 , last page 4
0969-997X

0969-997X

0969-997X

0969-997X

1065-6995

1065-6995

1065-6995

0967-0645

0307-6962

0022-104X

0046-225X

0304-3770

0304-3770

0304-3770

0304-3770

0304-3770

0304-3770

0304-3770

1027-5606

0033-183X

0033-183X

0164-0291

0164-0291

0026-6493

0026-6493

0026-6493

0026-6493

0141-8130

1146-609X

1146-609X

1146-609X

1146-609X

1146-609X

0003-455X

0022-3395

0022-3395

0022-3395

0967-0262

0377-8401

1286-4560

0013-8746

0013-8746

0013-8746

0013-8746

0013-8746

0013-8746

1445-5226

1445-5226

0340-2061

0266-4674

0266-4674

0266-4674

0266-4674

0266-4674

0266-4674

0266-4674

IF Half-Life

$1.371 \quad 4.1$

$1.371 \quad 4.1$

$1.371 \quad 4.1$

$1.371 \quad 4.1$

$1.363 \quad 6$

$1.363 \quad 6$

1.3636

$1.358 \quad 5.2$

$1.355 \quad 10$

$1.354 \quad 2.6$

$1.344 \quad 10$

$1.338 \quad 10$

$1.338 \quad 10$

$1.338 \quad 10$

$1.338 \quad 10$

$1.338 \quad 10$

$1.338 \quad 10$

$1.338 \quad 10$

$\begin{array}{ll}1.333 & 4.7\end{array}$

$1.333 \quad 10$

$1.333 \quad 10$

$\begin{array}{ll}1.331 & 7.9\end{array}$

$1.331 \quad 7.9$

$1.328 \quad 10$

$1.328 \quad 10$

$1.328 \quad 10$

$1.328 \quad 10$

$1.323 \quad 8.3$

$1.32 \quad 7.5$

$1.32 \quad 7.5$

$1.32 \quad 7.5$

$1.32 \quad 7.5$

$1.32 \quad 7.5$

$1.316 \quad 10$

$\begin{array}{ll}1.3 & 9.8\end{array}$

$1.3 \quad 9.8$

$1.3 \quad 9.8$

$1.293 \quad 5.9$

$1.29 \quad 8$

1.294

$1.289 \quad 10$

1.289

$1.289 \quad 10$

$1.289 \quad 10$

$1.289 \quad 10$

$1.289 \quad 10$

$1.28 \quad 3.4$

$1.28 \quad 3.4$

$1.277 \quad 10$

$1.277 \quad 8.3$

$1.277 \quad 8.3$

$1.277 \quad 8.3$

$1.277 \quad 8.3$

$1.277 \quad 8.3$

$1.277 \quad 8.3$

$1.277 \quad 8.3$ 


\begin{tabular}{|c|c|c|c|c|}
\hline Journal & Paper info. & ISSN & IF & Half-Life \\
\hline Canadian Journal of Microbiology & v. 52 , fisrt page 575 , last page 583 & $0008-4166$ & 1.275 & 10 \\
\hline Journal of Economic Entomology & v. 97, fisrt page 741 , last page 747 & $0022-0493$ & 1.275 & 10 \\
\hline Hereditas (Lund) & v. 140 , fistt page 149 , last page 153 & $0018-0661$ & 1.269 & 10 \\
\hline Hereditas (Lund) & v. 140 , fistt page 18 , last page 23 & $0018-0661$ & 1.269 & 10 \\
\hline Hereditas (Lund) & v. 140, fistt page 87, last page 91 & $0018-0661$ & 1.269 & 10 \\
\hline Hereditas (Lund) & v. 141 , fistt page 318 , last page 322 & $0018-0661$ & 1.269 & 10 \\
\hline Hereditas (Lund) & v. 141 , fistt page 89, last page 93 & $0018-0661$ & 1.269 & 10 \\
\hline Hereditas (Lund) & v. 142, fisrt page 1 , last page 5 & $0018-0661$ & 1.269 & 10 \\
\hline Chemical and Pharmaceutical Bulletin & v. 52, fisrt page 1433 , last page 1435 & $0009-2363$ & 1.262 & 10 \\
\hline Research in Veterinary Science & v. 80 , fisrt page 209 , last page 217 & $0034-5288$ & 1.258 & 10 \\
\hline Research in Veterinary Science & v. 81 , fisrt page 246 , last page 253 & $0034-5288$ & 1.258 & 10 \\
\hline Cereal Chemistry & v. 81 , fisrt page 115 , last page 127 & 0009-0352 & 1.254 & 10 \\
\hline Aquatic Living Resources & v. 17, fisrt page 19 , last page 23 & $0990-7440$ & 1.247 & 7.4 \\
\hline Aquatic Living Resources & v. 17 , fisrt page 215 , last page 229 & $0990-7440$ & 1.247 & 7.4 \\
\hline Aquatic Living Resources & v. 18 , fisrt page 169 , last page 178 & $0990-7440$ & 1.247 & 7.4 \\
\hline Plant Systematics and Evolution & v. 2004 , fisrt page 241 , last page 254 & 0378-2697 & 1.239 & 8 \\
\hline Plant Systematics and Evolution & v. 244 , fistt page 123 , last page 125 & 0378-2697 & 1.239 & 8 \\
\hline Plant Systematics and Evolution & v. 245 , fisrt page 41 , last page 54 & 0378-2697 & 1.239 & 8 \\
\hline Plant Systematics and Evolution & v. 247 , fisrt page 37 , last page 60 & 0378-2697 & 1.239 & 8 \\
\hline Plant Systematics and Evolution & v. 250 , fisrt page 147 , last page 156 & $0378-2697$ & 1.239 & 8 \\
\hline Plant Systematics and Evolution & v. 253 , fisrt page 1 , last page 22 & 0378-2697 & 1.239 & 8 \\
\hline Plant Systematics and Evolution & v. 253 , fisrt page 65 , last page 80 & $0378-2697$ & 1.239 & 8 \\
\hline Plant Systematics and Evolution & v. 254 , fist page 131 , last page 148 & $0378-2697$ & 1.239 & 8 \\
\hline Plant Systematics and Evolution & v. 257 , fist page 119 , last page 127 & 0378-2697 & 1.239 & 8 \\
\hline Plant Systematics and Evolution & v. 258 , fisrt page 49 , last page 61 & 0378-2697 & 1.239 & 8 \\
\hline Plant Systematics and Evolution & v. 258 , fistt page 85 , last page 95 & 0378-2697 & 1.239 & 8 \\
\hline Plant Systematics and Evolution & v. 258 , fisrt page 85, last page 95 & 0378-2697 & 1.239 & 8 \\
\hline Plant Systematics and Evolution & v. 274 , fisrt page 215 , last page 231 & 0378-2697 & 1.239 & 8 \\
\hline Plant Systematics and Evolution, SBB & v. 247 , fisrt page 241 , last page 254 & $0378-2697$ & 1.239 & 8 \\
\hline Journal of Arid Environments & v. 62 , fisrt page 127 , last page 142 & $0140-1963$ & 1.238 & 6.9 \\
\hline Journal of Arid Environments & v. 62 , fisrt page 491 , last page 506 & 0140-1963 & 1.238 & 6.9 \\
\hline Journal of Invertebrate Pathology & v. 1, fisrt page 60 , last page 62 & $0022-2011$ & 1.235 & 10 \\
\hline Journal of Invertebrate Pathology & v. 85 , fisrt page 188 , last page 191 & $0022-2011$ & 1.235 & 10 \\
\hline Journal of Invertebrate Pathology & v. 88 , fisrt page 177 , last page 179 & $0022-2011$ & 1.235 & 10 \\
\hline Journal of Invertebrate Pathology & v. 91, fisrt page 199, last page 201 & $0022-2011$ & 1.235 & 10 \\
\hline Marine Mammal Science & v. 20 , fisrt page 145 , last page 151 & 0824-0469 & 1.235 & 7.6 \\
\hline Marine Mammal Science & v. 21 , fisrt page 150, last page 153 & 0824-0469 & 1.235 & 7.6 \\
\hline Flora (Jena) & v. 199 , fisrt page 47 , last page 57 & $0367-2530$ & 1.232 & 10 \\
\hline Flora (Jena) & v. 200, fistt page 119 , last page 135 & $0367-2530$ & 1.232 & 10 \\
\hline Flora (Jena) & v. 200 , fisrt page 456 , last page 476 & $0367-2530$ & 1.232 & 10 \\
\hline Flora (Jena) & v. 200 , fisrt page 65 , last page 73 & $0367-2530$ & 1.232 & 10 \\
\hline Flora (Jena) & v. 201, fisrt page 127 , last page 134 & $0367-2530$ & 1.232 & 10 \\
\hline Flora (Jena) & v. 201, fistt page 178 , last page 188 & $0367-2530$ & 1.232 & 10 \\
\hline Flora (Jena) & v. 201, fisrt page 606, last page 611 & $0367-2530$ & 1.232 & 10 \\
\hline Flora (Jena) & v. 38 , fisrt page 440 , last page 450 & $0367-2530$ & 1.232 & 10 \\
\hline Phytochemical Analsis & v. 15 , fisrt page 125, last page 129 & 0958-0344 & 1.228 & 5.9 \\
\hline Phytochemical Analysis & v. 16 , fisrt page 278 , last page 281 & 0958-0344 & 1.228 & 5.9 \\
\hline Molecular Ecology Notes & v. 4, fisrt page 406 , last page 408 & $1471-8278$ & 1.22 & 3.3 \\
\hline Molecular Ecology Notes & v. 4 , fistt page 575 , last page 577 & $1471-8278$ & 1.22 & 3.3 \\
\hline Molecular Ecology Notes & v. 6 , fistt page 307 , last page 309 & $1471-8278$ & 1.22 & 3.3 \\
\hline Molecular Ecology Notes & v. 6 , fisrt page 686 , last page 688 & $1471-8278$ & 1.22 & 3.3 \\
\hline Molecular Ecology Notes & v. n. 1 , fistt page 463 , last page 465 & $1471-8278$ & 1.22 & 3.3 \\
\hline European Journal of Plant Pathology & v. 110 , fistt page 861 , last page 865 & $0929-1873$ & 1.217 & 5.6 \\
\hline European Journal of Plant Pathology & v. 116 , fistt page 95 , last page 101 & $0929-1873$ & 1.217 & 5.6 \\
\hline Fisheries Research & v. 2170, fisrt page 1 , last page 6 & $0165-7836$ & 1.216 & 6.1 \\
\hline Fisheries Research & v. 66 , fisrt page 157, last page 169 & $0165-7836$ & 1.216 & 6.1 \\
\hline Fisheries Research & v. 66, fisrt page 19 , last page 30 & $0165-7836$ & 1.216 & 6.1 \\
\hline Fisheries Research & v. 69 , fisrt page 157 , last page 170 & $0165-7836$ & 1.216 & 6.1 \\
\hline Fisheries Research & v. 72 , fisrt page 271 , last page 277 & $0165-7836$ & 1.216 & 6.1 \\
\hline Fisheries Research & v. 73 , fisrt page 251 , last page 257 & $0165-7836$ & 1.216 & 6.1 \\
\hline
\end{tabular}




\begin{tabular}{|c|c|c|c|c|}
\hline $\begin{array}{r}\text { Journal } \\
\end{array}$ & Paper info. & ISSN & IF & Half-Life \\
\hline Fisheries Research & v. 74 , fisrt page 116 , last page 126 & $0165-7836$ & 1.216 & 6.1 \\
\hline Fisheries Research & v. 79 , fisrt page 251 , last page 257 & 0165-7836 & 1.216 & 6.1 \\
\hline Fisheries Research & v. 80 , fisrt page 196 , last page 202 & $0165-7836$ & 1.216 & 6.1 \\
\hline Fisheries Research & v. 81 , fisrt page 331 , last page 336 & $0165-7836$ & 1.216 & 6.1 \\
\hline Zoomorphology (Berlin) & v. 1 , fisrt page 135 , last page 145 & $0720-213 X$ & 1.211 & 10 \\
\hline Zoomorphology (Berlin) & v. 125 , fisrt page 135 , last page 145 & $0720-213 X$ & 1.211 & 10 \\
\hline Current Eye Research & v. 30 , fisrt page 405 , last page 413 & $0271-3683$ & 1.208 & 9.3 \\
\hline Memórias do Instituto Oswaldo Cruz & v. 100 , fistt page 161 , last page 167 & 0074-0276 & 1.208 & 6.7 \\
\hline Memórias do Instituto Oswaldo Cruz & v. 100 , fisrt page 249 , last page 257 & 0074-0276 & 1.208 & 6.7 \\
\hline Memórias do Instituto Oswaldo Cruz & v. 100 , fisrt page 39, last page 42 & 0074-0276 & 1.208 & 6.7 \\
\hline Memórias do Instituto Oswaldo Cruz & v. 100 , fisrt page 431 , last page 434 & 0074-0276 & 1.208 & 6.7 \\
\hline Memórias do Instituto Oswaldo Cruz & v. 100 , fistt page 441 , last page 444 & 0074-0276 & 1.208 & 6.7 \\
\hline Memórias do Instituto Oswaldo Cruz & v. 100 , fistt page 471 , last page 473 & 0074-0276 & 1.208 & 6.7 \\
\hline Memórias do Instituto Oswaldo Cruz & v. 100 , fisrt page 527 , last page 530 & 0074-0276 & 1.208 & 6.7 \\
\hline Memórias do Instituto Oswaldo Cruz & v. 100 , fistt page 631 , last page 637 & 0074-0276 & 1.208 & 6.7 \\
\hline Memórias do Instituto Oswaldo Cruz & v. 100 , fisrt page 739 , last page 741 & 0074-0276 & 1.208 & 6.7 \\
\hline Memórias do Instituto Oswaldo Cruz & v. 100 , fisrt page 783 , last page 787 & 0074-0276 & 1.208 & 6.7 \\
\hline Memórias do Instituto Oswaldo Cruz & v. 100 , fisrt page 833 , last page 839 & 0074-0276 & 1.208 & 6.7 \\
\hline Memórias do Instituto Oswaldo Cruz & v. 101 , fist page 113 , last page 115 & 0074-0276 & 1.208 & 6.7 \\
\hline Memórias do Instituto Oswaldo Cruz & v. 101 , fisrt page 175 , last page 193 & 0074-0276 & 1.208 & 6.7 \\
\hline Memórias do Instituto Oswaldo Cruz & v. 101 , fisrt page 179 , last page 184 & 0074-0276 & 1.208 & 6.7 \\
\hline Memórias do Instituto Oswaldo Cruz & v. 101, fisrt page 46 , last page 54 & 0074-0276 & 1.208 & 6.7 \\
\hline Memórias do Instituto Oswaldo Cruz & v. 101, fisrt page 669, last page 672 & 0074-0276 & 1.208 & 6.7 \\
\hline Memórias do Instituto Oswaldo Cruz & v. 101 , fistt page 851 , last page 856 & 0074-0276 & 1.208 & 6.7 \\
\hline Memórias do Instituto Oswaldo Cruz & v. 101, fistt page 99, last page 101 & 0074-0276 & 1.208 & 6.7 \\
\hline Memórias do Instituto Oswaldo Cruz & v. 99, fisrt page 27 , last page 36 & 0074-0276 & 1.208 & 6.7 \\
\hline Memórias do Instituto Oswaldo Cruz & v. 99, fisrt page 45 , last page 52 & 0074-0276 & 1.208 & 6.7 \\
\hline Memórias do Instituto Oswaldo Cruz & v. 99 , fisrt page 571 , last page 574 & 0074-0276 & 1.208 & 6.7 \\
\hline Memórias do Instituto Oswaldo Cruz & v. 99, fisrt page 597, last page 601 & 0074-0276 & 1.208 & 6.7 \\
\hline Memórias do Instituto Oswaldo Cruz & v. 99 , fisrt page 609 , last page 615 & 0074-0276 & 1.208 & 6.7 \\
\hline Memórias do Instituto Oswaldo Cruz & v. 99, fisrt page 73, last page 78 & 0074-0276 & 1.208 & 6.7 \\
\hline Water, Air and Soil Pollution & v. 175 , fisrt page 241 , last page 256 & $0049-6979$ & 1.205 & 9.1 \\
\hline Water, Air and Soil Pollution & v. DOI 101007 , fisrt page 11 , last page 15 & $0049-6979$ & 1.205 & 9.1 \\
\hline Water, Air and Soil Pollution, Maine System & v. 168 , fisrt page 129 , last page 143 & $0049-6979$ & 1.205 & 9.1 \\
\hline Micron & v. 37, fisrt page 223 , last page 228 & 0047-7206 & 1.2 & 5.4 \\
\hline Micron & v. 37 , fisrt page 234 , last page 242 & $0047-7206$ & 1.2 & 5.4 \\
\hline Micron & v. 37 , fisrt page 551 , last page 556 & $0047-7206$ & 1.2 & 5.4 \\
\hline Micron (Oxford) & v. 36 , fisrt page 155 , last page 161 & $0968-4328$ & 1.2 & 5.4 \\
\hline Micron (Oxford) & v. 36, fisrt page 89 , last page 93 & $0968-4328$ & 1.2 & 5.4 \\
\hline Micron (Oxford) & v. 37 , fisrt page 1 , last page 6 & $0968-4328$ & 1.2 & 5.4 \\
\hline Biologia Plantarum & v. 47 , fisrt page 53 , last page 59 & 0006-3134 & 1.198 & 4.7 \\
\hline Biologia Plantarum & v. 47 , fisrt page 67 , last page 70 & 0006-3134 & 1.198 & 4.7 \\
\hline Biologia Plantarum & v. 48, fisrt page 309 , last page 312 & 0006-3134 & 1.198 & 4.7 \\
\hline Biologia Plantarum & v. 48 , fisrt page 67 , last page 72 & 0006-3134 & 1.198 & 4.7 \\
\hline Biologia Plantarum & v. 49 , fisrt page 285 , last page 288 & 0006-3134 & 1.198 & 4.7 \\
\hline Biologia Plantarum & v. 50 , fisrt page 405 , last page 410 & 0006-3134 & 1.198 & 4.7 \\
\hline Folia Geobotanica et Phytotaxonomica & v. 39 , fisrt page 371 , last page 384 & $1211-9520$ & 1.196 & 8.7 \\
\hline Phycologia & v. 43 , fisrt page 329 , last page 340 & $0031-8884$ & 1.196 & 9.5 \\
\hline Phycologia & v. 43, fisrt page 50 , last page 57 & $0031-8884$ & 1.196 & 9.5 \\
\hline Phycologia & v. 44 , fisrt page 113 , last page 115 & $0031-8884$ & 1.196 & 9.5 \\
\hline Phycologia & v. 44 , fisrt page 212 , last page 221 & $0031-8884$ & 1.196 & 9.5 \\
\hline Phycologia & v. 44 , fisrt page 409 , last page 415 & $0031-8884$ & 1.196 & 9.5 \\
\hline Phycologia & v. 45 , fisrt page 432 , last page 441 & $0031-8884$ & 1.196 & 9.5 \\
\hline Canadian Journal of Botany & v. 82 , fisrt page 1419 , last page 1428 & $0008-4026$ & 1.193 & 10 \\
\hline Canadian Journal of Botany & v. 83 , fisrt page 1305 , last page 1316 & $0008-4026$ & 1.193 & 10 \\
\hline Canadian Journal of Botany & v. 83, fisrt page 279 , last page 286 & $0008-4026$ & 1.193 & 10 \\
\hline $\begin{array}{l}\text { Nuclear Instruments \& Methods in Physics } \\
\text { Research A }\end{array}$ & v. 12, fisrt page 2 , last page 8 & 0168-9002 & 1.185 & \\
\hline Nuclear Instruments \& Methods in Physics & v. 548 , fisrt page 207 , last page 212 & 0168-9002 & & \\
\hline $\begin{array}{l}\text { Research A } \\
\text { Nuclear Instruments \& Methods in Physics }\end{array}$ & v. 734 , fistt page 116 , last page 119 & 0168-9002 & $\begin{array}{l}1.185 \\
1.185\end{array}$ & $\begin{array}{l}5.4 \\
5.4\end{array}$ \\
\hline
\end{tabular}


Applied Animal Behaviour Science

Applied Animal Behaviour Science

Biological Research

Écoscience (Sainte-Foy)

Chromatographia (Wiesbaden)

Behaviour (Leiden)

Acta Protozoologica

Acta Protozoologica

Crop Science

Revista Geológica de Chile

Phytotherapy Research

Phytotherapy Research

Phytotherapy Research

Phytotherapy Research

Parasitology Research

Parasitology Research

Parasitology Research

Parasitology Research

Parasitology Research

Parasitology Research

Parasitology Research

Journal of Bioscience and Bioengineering

Oryx

Oryx

Biotechnology Letters

Human Biology

Human Biology

Protein and Peptide Letters

Protein and Peptide Letters

Environmental Toxicology and

Pharmacology

Environmental Toxicology and

Pharmacology

American Museum Novitates

American Museum Novitates

American Museum Novitates

American Museum Novitates

American Museum Novitates

American Museum Novitates

American Museum Novitates

American Museum Novitates

American Museum Novitates

Applied Biochemistry and Biotechnology

Environmental Management

Tissue \& Cell

Tissue \& Cell

Tissue \& Cell

Tissue \& Cell

Tissue \& Cell

Bulletin of Marine Science

Bulletin of Marine Science

Bulletin of Marine Science

Bulletin of Marine Science

African Journal of Marine Science

Brazilian Journal of Medical and Biological

Research

Brazilian Journal of Medical and Biological Research

Brazilian Journal of Medical and Biological Research v. 102 , fisrt page 329 , last page 343

0168-1591

0168-1591

v. 39, fisrt page 341, last page 352

v. 12 , n. Suplementa, fist page 136 , last page 147

v. 61 , fisrt page 291, last page 297

v. 143 , fisrt page 347 , last page 364

v. 4, fisrt page 351 , last page 362

v. 45 , fist page 77 , last page 90

v. 46, fist page 1546, last page 1552

v. 32, fist page 301 , last page 319

v. 18 , fisrt page 463 , last page 467

v. 19 , fisrt page 519 , last page 525

v. 19 , fisrt page 885 , last page 887

v. 19 , fisrt page 946 , last page 950

v. 92 , fisrt page 110 , last page 112

v. 93, fisrt page 230 , last page 234

v. 94 , fisrt page 201 , last page 206

v. 94 , fisrt page 471 , last page 473

v. 97 , fisrt page 171 , last page 173

v. 97 , fist page 94 , last page 97

v. 98 , fisrt page 295 , last page 298

v. 101 , fisrt page 287 , last page 296

v. 38 , fisrt page 385 , last page 394

v. 40 , fisrt page 218 , last page 224

v. 28 , fisrt page 447 , last page 453

v. 76 , fist page 591, last page 604

v. 78 , fist page 29 , last page 41

v. 13 , fist page 517 , last page 523

v. 13 , fist page 82 , last page 89

v. 19, fisrt page 197, last page 201

v. 21 , fist page 61 , last page 69

v. 3427 , fisrt page 1 , last page 12

v. 3437 , fist page 1 , last page 23

v. 3467 , fist page 1 , last page 24

v. 3496, fist page 1 , last page 27

v. 3501, fisrt page 1, last page 54

v. 3501 , fist page 54 , last page 60

v. 3512 , fisrt page 1 , last page 40

v. 3528 , fist page 1 , last page 11

v. 3537 , fist page 1 , last page 29

v. 113 , fisrt page 189 , last page 200

v. 38, fisrt page 974, last page 982

v. 1 , fisrt page 177 , last page 191

v. 1 , fist page 221 , last page 231

v. 37 , fisrt page 413 , last page 422

v. 37 , fisrt page 91 , last page 100

v. 38 , fisrt page 303 , last page 310

v. 73 , fisrt page 713 , last page 724

v. 73 , fisrt page 725 , last page 742

v. 77 , fisrt page 1 , last page 18

v. 79 , fisrt page 659 , last page 666

v. 27 , fisrt page 439 , last page 448

v. 37 , fisrt page 1 , last page 5

v. 37, fisrt page 1463, last page 1472

v. 37 , fisrt page 459 , last page 478
0716-9760

$1195-6860$

0009-5893

0005-7959

0065-1583

0065-1583

0011-183X

0716-0208

0951-418X

0951-418X

0951-418X

0951-418X

0932-0113

0932-0113

0932-0113

0932-0113

0932-0113

0932-0113

0932-0113

1389-1723

0030-6053

0030-6053

0141-5492

0018-7143

0018-7143

0929-8665

0929-8665

1382-6689

1382-6689

0003-0082

0003-0082

0003-0082

0003-0082

0003-0082

0003-0082

0003-0082

0003-0082

0003-0082

0273-2289

0364-152X

0040-8166

0040-8166

0040-8166

0040-8166

0040-8166

0007-4977

0007-4977

0007-4977

0007-4977

1814-232X

$0100-879 x$

0100-879X

0100-879X
1.177

1.177

1.177

1.174

1.171

9.1

9.1

4.8

7.5

7.1

10

5.8

5.8

10

8.7

5.4

5.4

5.4

5.4

5.4

5.4

5.4

5.4

5.4

5.4

5.4

4.9

4.8

4.8

6.3

10

10

2.8

2.8

$1.119 \quad 4.1$

1.119

4.1

10

10

10

10

10

10

10

10

10

7.3

6.8

10

10

10

10

10

10

10

10

10

2.5

1.075

6.4

1.075

6.4

1.075

6.4 


\begin{tabular}{|c|c|c|c|c|}
\hline Journal & Paper info. & ISSN & IF & Half-Life \\
\hline $\begin{array}{l}\text { Brazilian Journal of Medical and Biological } \\
\text { Research }\end{array}$ & v. 37 , fisrt page 479 , last page 483 & $0100-879 X$ & 1.075 & 6.4 \\
\hline Brazilian Journal of Medical and Biological & v. 38 , fisrt page 1669 , last page 1675 & $0100-879 x$ & & \\
\hline Research & & & 1.075 & 6.4 \\
\hline $\begin{array}{l}\text { Brazilian Journal of Medical and Biological } \\
\text { Research }\end{array}$ & v. 38 , fisrt page 1769 , last page 1773 & $0100-879 x$ & 1075 & 6.4 \\
\hline Brazilian Journal of Medical and Biological & v. 38 , fisrt page 885 , last page 893 & $0100-879 x$ & & \\
\hline Research & & & 1.075 & 6.4 \\
\hline $\begin{array}{l}\text { Brazilian Journal of Medical and Biological } \\
\text { Research }\end{array}$ & v. 39, fisrt page 1605, last page 1612 & 0100-879X & 1.075 & 6.4 \\
\hline $\begin{array}{l}\text { Brazilian Journal of Medical and Biological } \\
\text { Research }\end{array}$ & v. 39 , fisrt page 801 , last page 807 & $0100-879 x$ & 1075 & 64 \\
\hline Surgical Neurology & v. 65 , fistt page 12 , last page 19 & 0090-3019 & 1.057 & $\begin{array}{r}0.4 \\
10\end{array}$ \\
\hline Surgical Neurology & v. 66 , fisrt page 611 , last page 615 & 0090-3019 & 1.057 & 10 \\
\hline Primates & v. 45 , fisrt page 201 , last page 204 & $0032-8332$ & 1.053 & 10 \\
\hline Primates & v. 45, fisrt page 63 , last page 67 & $0032-8332$ & 1.053 & 10 \\
\hline Primates & v. 47 , fistt page 1 , last page 9 & $0032-8332$ & 1.053 & 10 \\
\hline Aquaculture Research & v. 36 , fisrt page 1049, last page 1055 & $1355-557 X$ & 1.051 & 5.3 \\
\hline Aquaculture Research & v. 36 , fisrt page 1069 , last page 1074 & $1355-557 X$ & 1.051 & 5.3 \\
\hline Aquaculture Research & v. 37 , fisrt page 1413 , last page 1418 & $1355-557 X$ & 1.051 & 5.3 \\
\hline Aquaculture Research & v. 37 , fisrt page 1516 , last page 1523 & $1355-557 X$ & 1.051 & 5.3 \\
\hline Aquaculture Research & v. 37 , fisrt page 1598 , last page 1600 & $1355-557 X$ & 1.051 & 5.3 \\
\hline Hydrobiologia (The Hague) & v. 1, fistt page 271, last page 284 & 0018-8158 & 1.049 & 92 \\
\hline Hydrobiologia (The Hague) & v. 2004 , fisrt page 1 , last page 12 & 0018-8158 & 1.049 & 9.2 \\
\hline Hydrobiologia (The Hague) & v. 5 , fistt page 1 , last page 13 & 0018-8158 & 1049 & 92 \\
\hline Hydrobiologia (The Hague) & v. 511 , fistt page 103 , last page 111 & 0018-8158 & 1049 & 92 \\
\hline Hydrobiologia (The Hague) & v. 513 , fistt page 183 , last page 196 & 0018-8158 & 1049 & 92 \\
\hline Hydrobiologia (The Hague) & v. 515 , fisrt page 37 , last page 48 & 0018-8158 & 1.049 & 9.2 \\
\hline Hydrobiologia (The Hague) & v. 518 , fisrt page 179 , last page 188 & 0018-8158 & 1.049 & 9.2 \\
\hline Hydrobiologia (The Hague) & v. 525, fisrt page 139 , last page 155 & $0018-8158$ & 1.049 & 9.2 \\
\hline Hydrobiologia (The Hague) & v. 529, fistt page 195, last page 203 & 0018-8158 & 1.049 & 9.2 \\
\hline Hydrobiologia (The Hague) & v. 530, fisrt page 223, last page 231 & 0018-8158 & 1.049 & 9.2 \\
\hline Hydrobiologia (The Hague) & v. $530-531$, fist page 347 , last page 355 & 0018-8158 & 1.049 & 9.2 \\
\hline Hydrobiologia (The Hague) & v. 541 , fistt page 71 , last page 85 & 0018-8158 & 1.049 & 9.2 \\
\hline Hydrobiologia (The Hague) & v. 542 , fisrt page 235 , last page 247 & 0018-8158 & 1.049 & 9.2 \\
\hline Hydrobiologia (The Hague) & v. 545, fisrt page 75 , last page 91 & 0018-8158 & 1.049 & 9.2 \\
\hline Hydrobiologia (The Hague) & v. 548, fisrt page 207, last page 215 & 0018-8158 & 1.049 & 9.2 \\
\hline Hydrobiologia (The Hague) & v. 549 , fisrt page 15 , last page 22 & 0018-8158 & 1.049 & 9.2 \\
\hline Hydrobiologia (The Hague) & v. 553, fisrt page 219 , last page 230 & 0018-8158 & 1.049 & 9.2 \\
\hline Hydrobiologia (The Hague) & v. 553, fisrt page 245 , last page 254 & 0018-8158 & 1.049 & 9.2 \\
\hline Hydrobiologia (The Hague) & v. 556, fisrt page 69 , last page 83 & 0018-8158 & 1.049 & 9.2 \\
\hline Hydrobiologia (The Hague) & v. 557 , fisrt page 69 , last page 77 & 0018-8158 & 1.049 & 9.2 \\
\hline Hydrobiologia (The Hague) & v. 560 , fisrt page 1 , last page 13 & 0018-8158 & 1.049 & 9.2 \\
\hline Hydrobiologia (The Hague) & v. 560 , fisrt page 393 , last page 404 & 0018-8158 & 1.049 & 9.2 \\
\hline Hydrobiologia (The Hague) & v. 570 , fisrt page 35 , last page 39 & 0018-8158 & 1.049 & 9.2 \\
\hline Hydrobiologia (The Hague) & v. 575 , fisrt page 69 , last page 81 & 0018-8158 & 1.049 & 9.2 \\
\hline Hydrobiologia (The Hague) & v. 575, fisrt page 83 , last page 94 & 0018-8158 & 1.049 & 9.2 \\
\hline Hydrobiologia (The Hague) & v. 59 , fisrt page 379 , last page 384 & 0018-8158 & 1.049 & 9.2 \\
\hline $\begin{array}{l}\text { Journal of the Science of Food and } \\
\text { Agriculture }\end{array}$ & v. 22, fistt page 1235, last page 1237 & $0022-5142$ & 1.026 & 9.2 \\
\hline Herpetologica (Austin) & v. 60 , fisrt page 245 , last page 260 & $0018-0831$ & 1.019 & 10 \\
\hline Herpetologica (Austin) & v. 60 , fisrt page 387 , last page 395 & $0018-0831$ & 1.019 & 10 \\
\hline Herpetologica (Austin) & v. 60, fisrt page 95, last page 101 & $0018-0831$ & 1.019 & 10 \\
\hline Herpetologica (Austin) & v. 61 , fistt page 209 , last page 218 & $0018-0831$ & 1.019 & 10 \\
\hline Herpetologica (Austin) & v. 62 , fistt page 367 , last page 379 & $0018-0831$ & 1.019 & 10 \\
\hline Ecological Research & v. 19 , fistt page 521 , last page 532 & $0912-3814$ & 1.012 & 5.5 \\
\hline Ecological Research & v. 21 , fistt page 117 , last page 123 & 0912-3814 & 1.012 & 5.5 \\
\hline Ecological Research & v. 21 , fistt page 150 , last page 156 & 0912-3814 & 1.012 & 5.5 \\
\hline Ecological Research & v. 21, fisrt page 617, last page 623 & 0912-3814 & 1.012 & 5.5 \\
\hline Ocean \& Coastal Management & v. 46 , fisrt page 1011 , last page 1030 & 0964-5691 & 1.011 & 6.3 \\
\hline Ocean \& Coastal Management & v. 49 , fisrt page 462 , last page 475 & 0964-5691 & 1.011 & 6.3 \\
\hline Geochemical Journal & v. 38 , fisrt page 255 , last page 264 & 0016-7002 & 1.01 & 9.2 \\
\hline Botanica Marina & v. 47 , fisrt page 147 , last page 151 & 0006-8055 & 1.009 & 10 \\
\hline
\end{tabular}




\begin{tabular}{|c|c|c|c|c|}
\hline Journal & Paper info. & ISSN & IF & Half-Life \\
\hline Botanica Marina & v. 47 , fisrt page 202 , last page 208 & 0006-8055 & 1.009 & 10 \\
\hline Botanica Marina & v. 47 , fisrt page 251 , last page 254 & 0006-8055 & 1.009 & 10 \\
\hline Botanica Marina & v. 48 , fisrt page 208 , last page 217 & 0006-8055 & 1.009 & 10 \\
\hline Botanica Marina & v. 49 , fisrt page 379 , last page 385 & 0006-8055 & 1.009 & 10 \\
\hline Journal of Sol-Gel Science and Technology & v. 38 , fisrt page 223 , last page 231 & 0928-0707 & 1.009 & 6 \\
\hline Current Microbiology & v. 50 , fisrt page 78 , last page 83 & 0343-8651 & 1.007 & 6.9 \\
\hline Current Microbiology & v. 51 , fisrt page 16 , last page 21 & 0343-8651 & 1.007 & 6.9 \\
\hline Current Microbiology & v. 53 , fisrt page 335 , last page 339 & $0343-8651$ & 1.007 & 6.9 \\
\hline Current Microbiology & v. 53, fisrt page 68, last page 71 & $0343-8651$ & 1.007 & 6.9 \\
\hline Scientia Marina & v. 64 , fisrt page 537 , last page 543 & 0214-8358 & 1.005 & 6.8 \\
\hline Scientia Marina & v. 68 , fisrt page 139 , last page 146 & 0214-8358 & 1.005 & 6.8 \\
\hline Scientia Marina & v. 69 , fisrt page 105, last page 112 & 0214-8358 & 1.005 & 6.8 \\
\hline Scientia Marina & v. 69 , fisrt page 199 , last page 204 & 0214-8358 & 1.005 & 6.8 \\
\hline Scientia Marina & v. 69, fisrt page 531 , last page 543 & 0214-8358 & 1.005 & 6.8 \\
\hline Scientia Marina & $\begin{array}{l}\text { v. } 70 \text {, n. supl } 3 \text {, fisrt page } 260 \text {, last page } \\
276\end{array}$ & 0214-8358 & 1.005 & 6.8 \\
\hline Scientia Marina & v. 70, fisrt page 189, last page 202 & 0214-8358 & 1.005 & 6.8 \\
\hline Scientia Marina & v. 70 , fisrt page 203 , last page 217 & 0214-8358 & 1.005 & 6.8 \\
\hline Scientia Marina & v. 70 , fisrt page 759 , last page 766 & 0214-8358 & 1.005 & 6.8 \\
\hline Journal of Food Science & v. 70, fisrt page 8 , last page 12 & $0022-1147$ & 1.004 & 10 \\
\hline Journal of the Brazilian Chemical Society & v. 15 , fisrt page 372 , last page 377 & 0103-5053 & 1.003 & 4.3 \\
\hline Journal of the Brazilian Chemical Society & v. 15, fisrt page 931 , last page 937 & 0103-5053 & 1.003 & 4.3 \\
\hline Journal of the Brazilian Chemical Society & v. 16 , fisrt page 1217 , last page 1225 & 0103-5053 & 1.003 & 4.3 \\
\hline Journal of the Brazilian Chemical Society & v. 16 , fisrt page 1387 , last page 1390 & 0103-5053 & 1.003 & 4.3 \\
\hline Journal of the Brazilian Chemical Society & v. 16, fisrt page 1391, last page 1395 & 0103-5053 & 1.003 & 4.3 \\
\hline Journal of the Brazilian Chemical Society & v. 16 , fisrt page 1431 , last page 1438 & 0103-5053 & 1.003 & 4.3 \\
\hline Journal of the Brazilian Chemical Society & v. 16 , fisrt page 1443 , last page 1447 & 0103-5053 & 1.003 & 4.3 \\
\hline Journal of the Brazilian Chemical Society & v. 16 , fisrt page 1463, last page 1466 & 0103-5053 & 1.003 & 4.3 \\
\hline Journal of the Brazilian Chemical Society & v. 16 , fisrt page 153 , last page 156 & 0103-5053 & 1.003 & 4.3 \\
\hline Journal of the Brazilian Chemical Society & v. 16 , fisrt page 654 , last page 656 & $0103-5053$ & 1.003 & 4.3 \\
\hline Journal of the Brazilian Chemical Society & v. 17 , fist page 1233 , last page 1240 & 0103-5053 & 1.003 & 4.3 \\
\hline Journal of the Brazilian Chemical Society & v. 17 , fisrt page 1409 , last page 1418 & 0103-5053 & 1.003 & 4.3 \\
\hline Journal of the Brazilian Chemical Society & v. 17 , fisrt page 542 , last page 547 & 0103-5053 & 1.003 & 4.3 \\
\hline Journal of the Brazilian Chemical Society & v. 17 , fisrt page 737 , last page 740 & 0103-5053 & 1.003 & 4.3 \\
\hline Journal of the Brazilian Chemical Society & v. 17 , fisrt page 803 , last page 806 & 0103-5053 & 1.003 & 4.3 \\
\hline Journal of the Brazilian Chemical Society & v. 17, fisrt page 807 , last page 811 & 0103-5053 & 1.003 & 4.3 \\
\hline Journal of Materials Science & v. 41 , fisrt page 2381 , last page 2386 & $0022-2461$ & 0.999 & 10 \\
\hline Real-Time Imaging & v. 10, fisrt page 239 , last page 250 & $1077-2014$ & 0.986 & 4.4 \\
\hline International Journal of Remote Sensing & v. 27, fisrt page 1539, last page 1562 & 0143-1161 & 0.98 & 8 \\
\hline Animal Welfare & v. 13 , fisrt page 225 , last page 232 & $0962-7286$ & 0.968 & 6.1 \\
\hline Journal of Molluscan Studies & v. 71 , fisrt page 199 , last page 210 & $0260-1230$ & 0.968 & 9.5 \\
\hline Journal of Molluscan Studies & v. 72 , fisrt page 218 , last page 220 & $0260-1230$ & 0.968 & 9.5 \\
\hline Chemoecology & v. 16 , fisrt page 203, last page 209 & 0937-7409 & 0.967 & 5.7 \\
\hline Journal of Insect Behavior & v. 17 , fisrt page 251 , last page 261 & $0892-7553$ & 0.967 & 8.6 \\
\hline Journal of Insect Behavior & v. 17 , fisrt page 263 , last page 271 & $0892-7553$ & 0.967 & 8.6 \\
\hline Journal of Insect Behavior & v. 18 , fisrt page 281 , last page 292 & $0892-7553$ & 0.967 & 8.6 \\
\hline Journal of Insect Behavior & v. 18 , fisrt page 433 , last page 452 & $0892-7553$ & 0.967 & 8.6 \\
\hline Journal of Insect Behavior & v. 18 , fisrt page 543 , last page 555 & $0892-7553$ & 0.967 & 8.6 \\
\hline Journal of Insect Behavior & v. 19 , fisrt page 717 , last page 729 & $0892-7553$ & 0.967 & 8.6 \\
\hline $\begin{array}{l}\text { Zeitschrift Für Naturforschung C-A,Journal } \\
\text { of Biosciences }\end{array}$ & v. $60 \mathrm{c}$, fisrt page 72 , last page 78 & $0250-5991$ & 0.966 & 4.7 \\
\hline Folia Microbiologica & v. 50 , fisrt page 421 , last page 425 & 0015-5632 & 0.963 & 6 \\
\hline Folia Microbiologica (Prague) & v. 49 , fisrt page 46 , last page 52 & 0015-5632 & 0.963 & 6 \\
\hline Mammalian Biology & v. 69 , fisrt page 430 , last page 433 & $1616-5047$ & 0.962 & 3.3 \\
\hline Mammalian Biology & v. 79 , fisrt page 366 , last page 375 & $1616-5047$ & 0.962 & 3.3 \\
\hline Transplantation Proceedings & v. 36 , fisrt page 1261 , last page 1264 & 0041-1345 & 0.962 & 5.9 \\
\hline Plant Breeding & v. 124 , fisrt page 572 , last page 575 & 0179-9541 & 0.954 & 7.1 \\
\hline Plant Cell, Tissue and Organ Culture & v. 78 , fisrt page 231 , last page 235 & 0167-6857 & 0.951 & 7.9 \\
\hline Plant Cell, Tissue and Organ Culture & v. 78, fisrt page 37 , last page 42 & 0167-6857 & 0.951 & 7.9 \\
\hline Plant Cell, Tissue and Organ Culture & v. 78 , fisrt page 51 , last page 53 & 0167-6857 & 0.951 & 7.9 \\
\hline Plant Cell, Tissue and Organ Culture & v. 80 , fisrt page 129 , last page 137 & $0167-6857$ & 0.951 & 7.9 \\
\hline
\end{tabular}


\begin{tabular}{c} 
Journal \\
\hline Plant Cell, Tissue and Organ Culture
\end{tabular}

Plant Cell, Tissue and Organ Culture

Plant Cell, Tissue and Organ Culture

Plant Cell, Tissue and Organ Culture

Journal of Systematic Paleontology

Journal of Thermal Biology

Aquaculture International

Aquaculture International

Aquaculture International

Zoological Studies

Zoological Studies

Zoological Studies

Australian Journal of Botany

Australian Journal of Botany

Australian Journal of Botany

Australian Journal of Botany

Australian Journal of Botany

Australian Journal of Botany

Australian Journal of Botany

Australian Journal of Botany

Australian Journal of Botany, NFT

Marine Ecology

Marine Ecology

Marine Ecology

Environmental Biology of Fishes

Environmental Biology of Fishes

Environmental Biology of Fishes

Environmental Biology of Fishes

Environmental Biology of Fishes

Environmental Biology of Fishes

Environmental Biology of Fishes

Environmental Biology of Fishes

Herpetological Journal

Herpetological Journal

Herpetological Journal

Herpetological Journal

Herpetological Journal

Herpetological Journal

Herpetological Journal

Herpetological Journal

Herpetological Journal

Herpetological Journal

Agroforestry Systems

Mycopathologia

Mycopathologia

Fitoterapia

Fitoterapia

Euphytica (Wageningen)

Euphytica (Wageningen)

Euphytica (Wageningen)

Euphytica (Wageningen)

Acta Zoologica (Stockholm)

Acta Zoologica (Stockholm)

Acta Zoologica (Stockholm)

Acta Zoologica (Stockholm)

Acta Zoologica (Stockholm)

Acta Zoologica (Stockholm)

Acta Zoologica (Stockholm)

Acta Zoologica (Stockholm)

Acta Zoologica (Stockholm)
Paper info.

v. 80 , fist page 69 , last page 85

v. 81 , fisrt page 101 , last page 104

v. 86 , fisrt page 211 , last page 218

v. 86 , fisrt page 329 , last page 335

v. 4 , fisrt page 109 , last page 118

v. 29 , fisrt page 315 , last page 324

v. 250 , fisrt page 283 , last page 290

v. 256 , fisrt page 521 , last page 528

v. 258, fisrt page 655, last page 663

v. 45 , fisrt page 20 , last page 40

v. 45 , fisrt page 223 , last page 233

v. 45 , fisrt page 285 , last page 303

v. 52 , fisrt page 1 , last page 7

v. 52 , fisrt page 149 , last page 161

v. 52 , fisrt page 677 , last page 683

v. 53 , fisrt page 273 , last page 279

v. 53 , fisrt page 789 , last page 796

v. 54 , fisrt page 315 , last page 324

v. 54 , fisrt page 409 , last page 415

v. 54 , fisrt page 755 , last page 764

v. 1 , fisrt page 156, last page 163

v. 25 , fisrt page 173 , last page 190

v. 25 , fisrt page 249 , last page 263

v. 27 , fisrt page 160, last page 169

v. 70 , fisrt page 293 , last page 304

v. 70 , fisrt page 415 , last page 425

v. 71, fisrt page 341 , last page 351

v. 72 , fisrt page 213 , last page 221

v. 75 , fisrt page 219 , last page 221

v. 75 , fisrt page 349 , last page 360

v. 77 , fisrt page 1 , last page 8

v. 77 , fisrt page 309 , last page 315

v. 15 , fisrt page 181, last page 189

v. 15 , fisrt page 195, last page 199

v. 15 , fisrt page 279 , last page 284

v. 16 , fisrt page 1 , last page 10

v. 16, fisrt page 122, last page 155

v. 16 , fisrt page 21 , last page 27

v. 16 , fist page 37 , last page 45

v. 35 , fisrt page 239 , last page 243

v. 62 , fisrt page 221 , last page 233

v. 62 , fisrt page 453 , last page 465

v. 61 , fisrt page 195, last page 206

v. 162 , fisrt page 115 , last page 119

v. 162 , fisrt page 337 , last page 346

v. 76 , fisrt page 494 , last page 496

v. 76 , fisrt page 755 , last page 757

v. 135 , fisrt page 99, last page 105

v. 138 , fisrt page 213 , last page 218

v. 138 , fist page 55 , last page 60

v. 143 , fisrt page 237 , last page 245

v. 1 , fisrt page 341 , last page 348

v. 39, fist page 1136, last page 1139

v. 39, fisrt page 1140, last page 1143

v. 86 , fisrt page 111 , last page 118

v. 86 , fisrt page 217 , last page 221

v. 86 , fisrt page 289 , last page 294

v. 86 , fist page 33 , last page 40

v. 87 , fisrt page 203, last page 207

v. 87 , fisrt page 253 , last page 264
ISSN

0167-6857

0167-6857

0167-6857

0167-6857

1477-2019

0306-4565

0967-6120

0967-6120

0967-6120

1021-5506

1021-5506

1021-5506

0067-1924

0067-1924

0067-1924

0067-1924

0067-1924

0067-1924

0067-1924

0067-1924

0067-1924

0173-9565

0173-9565

0173-9565

0378-1909

0378-1909

0378-1909

0378-1909

0378-1909

0378-1909

0378-1909

0378-1909

0268-0130

0268-0130

0268-0130

0268-0130

0268-0130

0268-0130

0268-0130

0268-0130

0268-0130

0268-0130

0167-4366

0301-486X

0301-486X

0367-326X

0367-326X

0014-2336

0014-2336

0014-2336

0014-2336

0001-7272

0001-7272

0001-7272

0001-7272

0001-7272

0001-7272

0001-7272

0001-7272

0001-7272

IF Half-Life

$0.951 \quad 7.9$

$0.951 \quad 7.9$

$0.951 \quad 7.9$

$0.951 \quad 7.9$

0.95

$0.95 \quad 7.1$

$0.943 \quad 6.1$

$0.943 \quad 6.1$

$0.943 \quad 6.1$

$0.943 \quad 4.4$

$0.943 \quad 4.4$

$0.943 \quad 4.4$

$0.94 \quad 10$

$0.94 \quad 10$

$0.94 \quad 10$

$0.94 \quad 10$

$0.94 \quad 10$

$0.94 \quad 10$

$0.94 \quad 10$

$0.94 \quad 10$

$0.94 \quad 10$

$0.936 \quad 10$

$0.936 \quad 10$

$0.936 \quad 10$

$0.934 \quad 9.9$

$0.934 \quad 9.9$

$0.934 \quad 9.9$

$0.934 \quad 9.9$

$0.934 \quad 9.9$

$0.934 \quad 9.9$

$0.934 \quad 9.9$

$0.934 \quad 9.9$

$0.924 \quad 7.7$

$0.924 \quad 7.7$

$0.924 \quad 7.7$

$\begin{array}{ll}0.924 & 7.7\end{array}$

$\begin{array}{ll}0.924 & 7.7\end{array}$

$0.924 \quad 7.7$

$\begin{array}{ll}0.924 & 7.7\end{array}$

$0.924 \quad 7.7$

$0.924 \quad 7.7$

$0.924 \quad 7.7$

$0.921 \quad 7.5$

$0.915 \quad 10$

$0.915 \quad 10$

$0.908 \quad 5.9$

$0.908 \quad 5.9$

$0.907 \quad 8.4$

$0.907 \quad 8.4$

$0.907 \quad 8.4$

$0.907 \quad 8.4$

$0.906 \quad 10$

$0.906 \quad 10$

$0.906 \quad 10$

$0.906 \quad 10$

$0.906 \quad 10$

$0.906 \quad 10$

$0.906 \quad 10$

$0.906 \quad 10$

$0.906 \quad 10$ 
Journal

Acta Zoologica (Stockholm)

Biochemical Systematics and Ecology

Biochemical Systematics and Ecology

Biochemical Systematics and Ecology

Biochemical Systematics and Ecology

Biochemical Systematics and Ecology

Biochemical Systematics and Ecology

Biochemical Systematics and Ecology

Biochemical Systematics and Ecology

Biochemical Systematics and Ecology

Biochemical Systematics and Ecology

Biochemical Systematics and Ecology

Biochemical Systematics and Ecology

Biochemical Systematics and Ecology

Biochemical Systematics and Ecology

Biochemical Systematics and Ecology

Biochemical Systematics and Ecology

Biochemical Systematics and Ecology

Biochemical Systematics and Ecology

Biochemical Systematics and Ecology

Biochemical Systematics and Ecology

Biochemical Systematics and Ecology

Biochemical Systematics and Ecology

Biochemical Systematics and Ecology

Biochemical Systematics and Ecology

Biochemical Systematics and Ecology

Biochemical Systematics and Ecology

Biochemical Systematics and Ecology

Folia Primatologica

Folia Primatologica

Folia Primatologica

Folia Primatologica

Folia Primatologica

Folia Primatologica

Plant Growth Regulation

Plant Growth Regulation

Plant Growth Regulation

Plant Growth Regulation

Aquatic Ecology

Journal of the American Mosquito Control

Association

Herpetological Monograph

Food Biotechnology

Journal of Chromatographic Science

Journal of Ethology

Journal of Ethology

Journal of Ethology

Journal of Ethology

Biochemical Genetics

European Journal of Soil Biology

Journal of Shellfish Research

Flavour and Fragrance Journal

Flavour And Fragrance Journal

Flavour and Fragrance Journal

Flavour and Fragrance Journal

Flavour and Fragrance Journal

Flavour and Fragrance Journal

Radiation Physics and Chemistry

Biocontrol Science and Technology
Paper info.

v. 87 , fisrt page 265 , last page 272

v. 87 , fisrt page 305 , last page 313

v. 01 , fisrt page 573 , last page 582

v. 27 , fisrt page 439 , last page 447

v. 29 , fisrt page 657 , last page 667

v. 32, fist page 1219, last page 1222

v. 32 , fisrt page 253 , last page 263

v. 32 , fist page 27 , last page 34

v. 32 , fisrt page 337 , last page 342

v. 32, fisrt page 503 , last page 505

v. 32 , fisrt page 747 , last page 753

v. 32 , fisrt page 817 , last page 821

v. 32 , fisrt page 893 , last page 899

v. 32 , fisrt page 931 , last page 935

v. 33 , fisrt page 1 , last page 16

v. 33, fist page 1210, last page 1225

v. 33 , fisrt page 233 , last page 243

v. 33 , fisrt page 269 , last page 274

v. 33 , fisrt page 331 , last page 334

v. 33 , fisrt page 537 , last page 540

v. 33 , fisrt page 675 , last page 679

v. 33 , fisrt page 971 , last page 981

v. 34 , fisrt page 263 , last page 264

v. 34 , fisrt page 265 , last page 267

v. 34, fisrt page 291, last page 302

v. 34 , fisrt page 446 , last page 448

v. 34 , fisrt page 78 , last page 82

v. 34 , fisrt page 822 , last page 824

v. 35 , fisrt page 479 , last page 486

v. 45, fisrt page 201, last page 204

v. 75 , fisrt page 11 , last page 113

v. 75 , fisrt page 154 , last page 156

v. 75 , fisrt page 335 , last page 338

v. 76 , fisrt page 112 , last page 115

v. 76 , fisrt page 33 , last page 36

v. $2 / 3$, fisrt page 237 , last page 247

v. 44 , fisrt page 125 , last page 131

v. 44 , fisrt page 147 , last page 156

v. 49 , fisrt page 237 , last page 247

v. 40 , fisrt page 381 , last page 390

v. 20 , fisrt page 350 , last page 356

v. 19 , fisrt page 137, last page 152

v. 20 , fist page 65 , last page 77

v. 44 , fisrt page 233 , last page 238

v. 23 , fist page 01 , last page 15

v. 23 , fisrt page 133 , last page 138

v. 24 , fisrt page 219 , last page 225

v. 6 , fisrt page 95, last page 100

v. 43 , fisrt page 335 , last page 345

v. 41 , fisrt page 99 , last page 107

v. 23, fisrt page 581 , last page 584

v. 1 , fisrt page 17 , last page 21

v. 19 , fisrt page 1 , last page 3

v. 19 , fisrt page 80 , last page 82

v. 20 , fisrt page 161 , last page 163

v. 20 , fisrt page 39 , last page 41

v. 20 , fisrt page 474 , last page 477

v. 71 , fisrt page 787 , last page 789

v. 14 , fisrt page 313 , last page 319
ISSN

0001-7272

0001-7272

0305-1978

0305-1978

0305-1978

0305-1978

0305-1978

0305-1978

0305-1978

0305-1978

0305-1978

0305-1978

0305-1978

0305-1978

0305-1978

0305-1978

0305-1978

0305-1978

0305-1978

0305-1978

0305-1978

0305-1978

0305-1978

0305-1978

0305-1978

0305-1978

0305-1978

0305-1978

0305-1978

0015-5713

0015-5713

0015-5713

0015-5713

0015-5713

$0015-5713$

0167-6903

0167-6903

0167-6903

0167-6903

1386-2588

8756-971X

0733-1347

0890-5436

0021-9665

0289-0771

0289-0771

0289-0771

0289-0771

0006-2928

1164-5563

0730-8000

0882-5734

0882-5734

$0882-5734$

$0882-5734$

0882-5734

0882-5734

0969-806X

0958-3157

IF

0.906

0.906

0.906

0.906

0.906

0.906

0.906

0.906

0.906

0.906

0.906

0.906

0.906

0.906

0.906

0.906

0.906

0.906

0.906

0.906

0.906

0.906

0.906

0.906

0.906

0.906

0.906

0.906

0.906

0.904

0.904

0.904

0.904

0.904

0.904

0.903

0.903

0.903

0.903

0.898

0.895

0.889

0.886

0.88

0.877

0.877

0.877

0.877

0.876

0.875

0.875

0.868

0.868

0.868

0.868

0.868

0.868

0.868

0.862

Half-Life

10

10

7.1

7.1

7.1

7.1

7.1

7.1

7.1

7.1

7.1

7.1

7.1

7.1

7.1

7.1

7.1

7.1

7.1

7.1

7.1

7.1

7.1

7.1

7.1

7.1

7.1

7.1

7.1

10

10

10

10

10

10

6.9

6.9

6.9

6.9

6

10

10

10

10

6.4

6.4

6.4

6.4

10 


\begin{tabular}{|c|c|c|c|c|}
\hline Journal & Paper info. & ISSN & IF & Half-Life \\
\hline Systematic Parasitology & v. 64 , fisrt page 54 , last page 68 & 0165-5752 & 0.856 & 8.5 \\
\hline Systematic Parasitology & v. 64 , fisrt page 57 , last page 68 & 0165-5752 & 0.856 & 8.5 \\
\hline Systematic Parasitology & v. 66, fistt page 1 , last page 34 & $0165-5752$ & 0.856 & 8.5 \\
\hline Systematic Parasitology & v. 666, fisrt page 35, last page 41 & $0165-5752$ & 0.856 & 8.5 \\
\hline Journal of Forensic Sciences & v. 51, fisrt page 1151, last page 1153 & $0022-1198$ & 0.846 & 8 \\
\hline Journal of Insect Science & v. 4 , fisrt page 1 , last page 3 & 0970-3837 & 0.844 & 3.6 \\
\hline Journal of Applied Entomology & v. 1 , fist page 204 , last page 211 & $0931-2048$ & 0.841 & 8.6 \\
\hline Journal of Applied Entomology & v. 1 , fisrt page 329 , last page 331 & 0931-2048 & 0.841 & 8.6 \\
\hline Journal of Applied Entomology & v. 128 , fistt page 112 , last page 119 & 0931-2048 & 0.841 & 8.6 \\
\hline Journal of Applied Entomology & v. 128 , fistt page 204 , last page 211 & 0931-2048 & 0.841 & 8.6 \\
\hline Journal of Applied Entomology & v. 128 , fistt page 279 , last page 283 & 0931-2048 & 0.841 & 8.6 \\
\hline Journal of Applied Entomology & v. 128 , fistt page 432 , last page 436 & 0931-2048 & 0.841 & 8.6 \\
\hline Journal of Applied Entomology & v. 128 , fistt page 88, last page 94 & 0931-2048 & 0.841 & 8.6 \\
\hline Journal of Applied Entomology & v. 129 , fistt page 347 , last page 351 & 0931-2048 & 0.841 & 8.6 \\
\hline Journal of Applied Entomology & v. 130 , fisrt page 56 , last page 60 & $0931-2048$ & 0.841 & 8.6 \\
\hline Journal of Applied Entomology & v. 130 , fisrt page 84 , last page 90 & $0931-2048$ & 0.841 & 8.6 \\
\hline Molecules (Basel) & v. 10 , fisrt page 1010, last page 1014 & $1420-3049$ & 0.841 & 4.4 \\
\hline Copeia & v. 01, fisrt page 496, last page 506 & $0045-8511$ & 0.84 & 10 \\
\hline Copeia & v. 1 , fisrt page 68 , last page 73 & $0045-8511$ & 0.84 & 10 \\
\hline Copeia & v. 2 , fisrt page 272 , last page 278 & 0045-8511 & 0.84 & 10 \\
\hline Copeia & v. 2004 , fisrt page 315 , last page 325 & $0045-8511$ & 0.84 & 10 \\
\hline Copeia & v. 2004 , fisrt page 336 , last page 343 & $0045-8511$ & 0.84 & 10 \\
\hline Copeia & v. 2004 , fisrt page 577 , last page 582 & $0045-8511$ & 0.84 & 10 \\
\hline Copeia & v. 2004 , fisrt page 665 , last page 676 & $0045-8511$ & 0.84 & 10 \\
\hline Copeia & v. 2005 , fisrt page 100 , last page 108 & $0045-8511$ & 0.84 & 10 \\
\hline Copeia & v. 2005 , fisrt page 386 , last page 392 & $0045-8511$ & 0.84 & 10 \\
\hline Copeia & v. 2005 , fisrt page 550 , last page 558 & $0045-8511$ & 0.84 & 10 \\
\hline Copeia & v. 2005, fisrt page 609, last page 631 & $0045-8511$ & 0.84 & 10 \\
\hline Copeia & v. 2005 , fisrt page 678 , last page 682 & $0045-8511$ & 0.84 & 10 \\
\hline Copeia & v. 2005 , fisrt page 764 , last page 770 & $0045-8511$ & 0.84 & 10 \\
\hline Copeia & v. 2005 , fisrt page 845 , last page 853 & $0045-8511$ & 0.84 & 10 \\
\hline Copeia & v. 2006 , fisrt page 225 , last page 229 & $0045-8511$ & 0.84 & 10 \\
\hline Copeia & v. 2006, fisrt page 412 , last page 422 & $0045-8511$ & 0.84 & 10 \\
\hline Copeia & v. 2006 , fisrt page 516 , last page 528 & $0045-8511$ & 0.84 & 10 \\
\hline Copeia & v. 2006, fisrt page 613 , last page 622 & $0045-8511$ & 0.84 & 10 \\
\hline Copeia & v. 3 , fisrt page 534 , last page 545 & $0045-8511$ & 0.84 & 10 \\
\hline Copeia & v. 3, fisrt page 609, last page 631 & $0045-8511$ & 0.84 & 10 \\
\hline Journal of Liquid Chromatography \& & v. 28, fisrt page 1985, last page 1992 & $1082-6076$ & & \\
\hline $\begin{array}{l}\text { Related Technologies } \\
\text { Journal of Liquid Chromatography \& }\end{array}$ & v. 28 , fisrt page 2041 , last page 2051 & $1082-6076$ & 0.825 & 8.5 \\
\hline Related Technologies & & & 0.825 & 8.5 \\
\hline $\begin{array}{l}\text { Journal of Liquid Chromatography \& } \\
\text { Related Technologies }\end{array}$ & v. 29 , fisrt page 1833 , last page 1841 & $1082-6076$ & 0825 & 85 \\
\hline Zeitschrift für Naturforschung. B, A Journal & v. 59 , fisrt page 134 , last page 139 & 0932-0776 & & 8.5 \\
\hline of Chemical Sciences & & & 0.825 & 10 \\
\hline $\begin{array}{l}\text { Zeitschrift für Naturforschung. B, A Journal } \\
\text { of Chemical Sciences }\end{array}$ & v. 60 , fisrt page 72 , last page 78 & 0932-0776 & 0.825 & 10 \\
\hline $\begin{array}{l}\text { Zeitschrift für Naturforschung. B, A Journal } \\
\text { of Chemical Sciences }\end{array}$ & v. 60 , fisrt page 787 , last page 791 & $0932-0776$ & 0.825 & 10 \\
\hline Mediators of Inflammation & v. 13, fisrt page 29 , last page 32 & $0962-9351$ & 0.819 & 5.1 \\
\hline Smithsonian Contributions to Zoology & v. 60 , fist page 933 , last page 938 & 0081-0282 & 0.815 & 5.9 \\
\hline Smithsonian Contributions to Zoology & v. 622, fisrt page 1 , last page 190 & 0081-0282 & 0.815 & 5.9 \\
\hline Journal of Applied Ichthyology & v. 20 , fisrt page 48 , last page 52 & 0175-8659 & 0.812 & 5.7 \\
\hline Journal of Applied Ichthyology & v. 21 , fisrt page 469 , last page 477 & 0175-8659 & 0.812 & 5.7 \\
\hline Journal of Applied Ichthyology & v. 21 , fisrt page 75 , last page 79 & 0175-8659 & 0.812 & 5.7 \\
\hline Journal of Applied Ichthyology & v. 21 , fisrt page 94 , last page 100 & 0175-8659 & 0.812 & 5.7 \\
\hline Journal of Applied Ichthyology & v. 22 , fisrt page 132 , last page 137 & 0175-8659 & 0.812 & 5.7 \\
\hline Journal of Applied Ichthyology & v. 22, fisrt page 189, last page 192 & 0175-8659 & 0.812 & 5.7 \\
\hline Journal of Applied Ichthyology & v. 22 , fisrt page 325 , last page 327 & $0175-8659$ & 0.812 & 5.7 \\
\hline Diatom Research & v. 21, fisrt page 22, last page 30 & $0269-249 x$ & 0.809 & 10 \\
\hline Botanical Journal of the Linnean Society & v. 144 , fist page 181 , last page 191 & $0024-4074$ & 0.805 & 7.8 \\
\hline Botanical Journal of the Linnean Society & v. 145 , fist page 119 , last page 127 & $0024-4074$ & 0.805 & 7.8 \\
\hline
\end{tabular}


Journal Botanical Journal of the Linnean Society Botanical Journal of the Linnean Society Botanical Journal of the Linnean Society Botanical Journal of the Linnean Society Botanical Journal of the Linnean Society Botanical Journal of the Linnean Society Botanical Journal of the Linnean Society Botanical Journal of the Linnean Society Botanical Journal of the Linnean Society Journal of Bryology

Journal of Microencapsulation

American Malacological Bulletin

American Malacological Bulletin

American Malacological Bulletin

Animal Research

Arkivoc

ARKIVOC

ARKIVOC

ARKIVOC

Natural Product Research

Natural Product Research

Natural Product Research

Amphibia-Reptilia

Amphibia-Reptilia

Amphibia-Reptilia

Amphibia-Reptilia

Amphibia-Reptilia

Amphibia-Reptilia

Amphibia-Reptilia

Amphibia-Reptilia

Amphibia-Reptilia

Amphibia-Reptilia

Amphibia-Reptilia

Amphibia-Reptilia

Amphibia-Reptilia

Amphibia-Reptilia

Journal of Herpetology

Journal of Herpetology

Journal of Herpetology

Journal of Herpetology

Journal of Herpetology

Journal of Herpetology

Journal of Herpetology

Journal of Herpetology

Journal of Herpetology

Journal of Herpetology

Journal of Herpetology

Journal of Herpetology

Journal of Herpetology

Environmental Monitoring and Assessment

Environmental Monitoring and Assessment

Environmental Monitoring and Assessment

Food Technology and Biotechnology

Journal of Plant Pathology

Ethology Ecology \& Evolution

European Journal of Entomology

European Journal of Entomology

Photosynthetica

Photosynthetica
Paper info.

v. 146, fisrt page 103, last page 106

v. 146 , fisrt page 247 , last page 250

v. 146 , fisrt page 57 , last page 70

v. 147 , fisrt page 235 , last page 238

v. 148 , fisrt page 157 , last page 173

v. 148 , fisrt page 427 , last page 431

v. 148 , fist page 73 , last page 75

v. 150 , fisrt page 429 , last page 439

v. 151 , fisrt page 271 , last page 277

v. 44 , fisrt page 365 , last page 373

v. 28 , fisrt page 133 , last page 138

v. 21 , fisrt page 371 , last page 384

v. 18 , fisrt page 143 , last page 155

v. 18 , fisrt page 71 , last page 78

v. 20 , fisrt page 43 , last page 48

v. 55 , fisrt page 153 , last page 164

v. 2004 , fisrt page 89 , last page 97

v. 6 , fisrt page 05 , last page 11

v. 6 , fisrt page 59 , last page 65

v. 6 , fisrt page 89 , last page 94

v. 1 , fisrt page 43 , last page 46

v. 19 , fisrt page 319 , last page 323

v. 20 , fisrt page 27 , last page 30

v. 25 , fisrt page 125 , last page 135

v. 25 , fisrt page 219 , last page 227

v. 25, fisrt page 381 , last page 393

v. 26 , fisrt page 211 , last page 221

v. 26 , fisrt page 25 , last page 31

v. 26 , fist page 33 , last page 38

v. 26 , fisrt page 527 , last page 533

v. 26 , fisrt page 87 , last page 92

v. 27 , fisrt page 207 , last page 217

v. 27 , fisrt page 303 , last page 308

v. 27 , fisrt page 37 , last page 46

v. 27 , fisrt page 481 , last page 489

v. 27 , fisrt page 489 , last page 495

v. 55 , fisrt page 63 , last page 68

v. 1 , fist page 121 , last page 131

v. 16 , fisrt page 171 , last page 177

v. 38, fisrt page 501, last page 509

v. 39 , fisrt page 1 , last page 3

v. 39 , fisrt page 455 , last page 464

v. 39 , fisrt page 541 , last page 546

v. 39, fisrt page 664 , last page 667

v. 40 , fisrt page 171 , last page 180

v. 40 , fisrt page 261 , last page 266

v. 40 , fisrt page 274 , last page 279

v. 40 , fisrt page 413 , last page 418

v. 40 , fisrt page 556, last page 559

v. 40 , fisrt page 7 , last page 15

v. 102 , fisrt page 349 , last page 357

v. 114 , fisrt page 1 , last page 25

v. 90 , fisrt page 121 , last page 133

v. 44 , fisrt page 519 , last page 523

v. 88 , fisrt page 226 , last page 228

v. 17 , fisrt page 105 , last page 119

v. 102 , fisrt page 201, last page 208

v. 102, fisrt page 691, last page 697

v. 42, fisrt page 291, last page 293

v. 43 , fisrt page 303 , last page 305
ISSN

0024-4074

0024-4074

0024-4074

0024-4074

0024-4074

0024-4074

0024-4074

0024-4074

0024-4074

0024-4074

0373-6687

0265-2048

0740-2783

0740-2783

0740-2783

1627-3583

1424-6376

1424-6376

$1424-6376$

1424-6376

1478-6419

1478-6419

1478-6419

0173-5373

0173-5373

0173-5373

0173-5373

0173-5373

0173-5373

0173-5373

0173-5373

0173-5373

0173-5373

0173-5373

0173-5373

0173-5373

0173-5373

0022-1511

0022-1511

0022-1511

0022-1511

0022-1511

0022-1511

0022-1511

0022-1511

0022-1511

0022-1511

0022-1511

0022-1511

0022-1511

0167-6369

0167-6369

0167-6369

1330-9862

1125-4653

0394-9370

1210-5759

1210-5759

0300-3604

0300-3604
Half-Life

$0.805 \quad 7.8$

$0.805 \quad 7.8$

$0.805 \quad 7.8$

$0.805 \quad 7.8$

$0.805 \quad 7.8$

$0.805 \quad 7.8$

$0.805 \quad 7.8$

$0.805 \quad 7.8$

$0.805 \quad 7.8$

$0.805 \quad 7.8$

$0.805 \quad 8$

$0.805 \quad 7.1$

$0.804 \quad 10$

$0.804 \quad 10$

$0.804 \quad 10$

$0.8 \quad 3.8$

$0.8 \quad 3.2$

$0.8 \quad 3.2$

$0.8 \quad 3.2$

$0.8 \quad 3.2$

$0.798 \quad 2.3$

$0.798 \quad 2.3$

$0.798 \quad 2.3$

$0.795 \quad 8.5$

$0.795 \quad 8.5$

$0.795 \quad 8.5$

$0.795 \quad 8.5$

$0.795 \quad 8.5$

$0.795 \quad 8.5$

$0.795 \quad 8.5$

$0.795 \quad 8.5$

$0.795 \quad 8.5$

$0.795 \quad 8.5$

$0.795 \quad 8.5$

$0.795 \quad 8.5$

$0.795 \quad 8.5$

$0.795 \quad 8.5$

$0.795 \quad 10$

$0.795 \quad 10$

$0.795 \quad 10$

$0.795 \quad 10$

$0.795 \quad 10$

$0.795 \quad 10$

$0.795 \quad 10$

$0.795 \quad 10$

$0.795 \quad 10$

$0.795 \quad 10$

$0.795 \quad 10$

$0.795 \quad 10$

$0.795 \quad 10$

$0.793 \quad 6$

$0.793 \quad 6$

$0.793 \quad 6$

$0.789 \quad 4.5$

$0.783 \quad 5.2$

$0.782 \quad 8.8$

$0.782 \quad 6.5$

$0.782 \quad 6.5$

$0.782 \quad 7.8$

$0.782 \quad 7.8$ 


\begin{tabular}{l}
\hline \multicolumn{1}{c}{ Journal } \\
\hline Photosynthetica \\
Photosynthetica \\
Zygote (Cambridge) \\
Botanical Review \\
Journal of Helminthology \\
Zoo Biology \\
Annals of Human Biology \\
Journal of the Marine Biological Association \\
of the United Kingdom \\
Journal of the Marine Biological Association \\
of the United Kingdom
\end{tabular}

Journal of the Marine Biological Association of the United Kingdom

Journal of the Marine Biological Association of the United Kingdom

Journal of the Marine Biological Association of the United Kingdom

Journal of the Marine Biological Association of the United Kingdom

Journal of the Marine Biological Association of the United Kingdom

Journal of the Marine Biological Association of the United Kingdom

Journal of the Marine Biological Association of the United Kingdom

Journal of the Marine Biological Association of the United Kingdom

Journal of the Marine Biological Association of the United Kingdom

Journal of the Marine Biological Association of the United Kingdom

Journal of the Marine Biological Association of the United Kingdom

Journal of the Marine Biological Association of the United Kingdom

Journal of the Marine Biological Association of the United Kingdom

Journal of the Marine Biological Association of the United Kingdom

Journal of the Marine Biological Association of the United Kingdom

Journal of the Marine Biological Association of the United Kingdom

Journal of the Marine Biological Association of the United Kingdom

Journal of the Marine Biological Association of the United Kingdom

International Review of Hydrobiology

Zoologischer Anzeiger

Zoologischer Anzeiger

Zoologischer Anzeiger

Acta Parasitologica

Acta Parasitologica

Acta Parasitologica

Applied Entomology and Zoology

Electronic Journal of Biotechnology

Animal Biology

Journal of the Hattori Botanical Laboratory

BioControl (Dordrecht)

Canadian Journal of Remote Sensing

Journal of Apicultural Research

Journal of Applied Phycology

Journal of Applied Phycology

Journal of Applied Phycology

Journal of Applied Phycology

Journal of Applied Phycology

Anais da Academia Brasileira de Ciências

Anais da Academia Brasileira de Ciências

v. 44, fisrt page 143, last page 146

v. 44 , fisrt page 235 , last page 240

v. 14 , fisrt page 217 , last page 229

v. 70 , fisrt page 357 , last page 380

v. 79 , fist page 75 , last page 84

v. 23 , fisrt page 85 , last page 89

v. 33 , fisrt page 142 , last page 160

v. 1 , fisrt page 5555 , last page 5566

v. 3, fisrt page 22 , last page 24

v. 84, fisrt page 1229, last page 1232

v. 84 , fisrt page 25 , last page 28

v. 84 , fisrt page 47 , last page 58

v. 84 , fisrt page 681 , last page 687

v. 84 , fisrt page 701 , last page 711

v. 84 , fisrt page 733 , last page 735

v. 84 , fisrt page 901 , last page 910

v. 84 , fisrt page 925 , last page 930

v. 84 , fisrt page 973 , last page 982

v. 85 , fisrt page 107 , last page 112

v. 85, fisrt page 1223, last page 1229

ISSN

0300-3604

IF

Half-Life

0300-3604

0.782

7.8

0967-1994

0.782

7.8

0006-8101

0.782

0022-149X

0.781

0733-3188

0.779

0301-4460

0.779

0025-3154

0.778

6.7

0025-3154

0.778

10

9.9

9.7

10

0025-3154

0.778

10

0025-3154

0025-3154

0.778

10

0025-3154

0.778

10

$0025-3154$

0.778

10

0025-3154

0.778

10

0025-3154

0.778

10

0025-3154

0.778

10

0025-3154

0.778

10

0025-3154

$0.778 \quad 10$

0025-3154

$0.778 \quad 10$

0025-3154

0.778

10

0025-3154

v. 85 , fisrt page 291 , last page 305

0.778

10

0025-3154

v. 85 , fisrt page 829 , last page 834

v. 86 , fistt page 1 , last page 5

v. 86, fisrt page 1449, last page 1458

v. 86 , fisrt page 227 , last page 233

v. 86 , fisrt page 861 , last page 875

v. 86 , fisrt page 957 , last page 961

v. 89 , fisrt page 317 , last page 325

243 v. 4 , fisrt page 273 , last page 279

v. 242 , fisrt page 353 , last page 365

v. 245 , fisrt page 127 , last page 129

v. 49 , fisrt page 353 , last page 361

v. 50 , fisrt page 150 , last page 155

v. 50 , fisrt page 210 , last page 216

v. 41 , fisrt page 419 , last page 427

v. 9, fisrt page 001 , last page 018

v. 54 , fisrt page 221 , last page 231

v. 98 , fisrt page 259, last page 298

v. 96 , fisrt page 109 , last page 114

v. 31, fisrt page 214, last page 224

v. 45 , fisrt page 112 , last page 116

v. 17 , fisrt page 447 , last page 460

v. 18 , fist page 1 , last page 9

v. 18 , fist page 83 , last page 93

v. 19 , fisrt page 140 , last page 148

v. 72 , fist page 1572 , last page 5176

0.778

10

0025-3154

0.778

10

0025-3154

0.778

10

0025-3154

$0.778 \quad 10$

0025-3154

0.778

10

0025-3154

0.778

10

0025-3154

1434-2944

0.778

10

0044-5231

0.775

5.3

0044-5231

0.775

0044-5231

0.775

$1230-2821$

0.775

$1230-2821$

0.772

0.772

1230-2821

0.772

0003-6862

0.77

0717-3458

0.767

1570-7555

0.759

0073-0912

0.755

1386-6141

0.754

0703-8992

0.752

0021-8839

0.75

0921-8971

0.746

0921-8971

0.746

0921-8971

0.746

0921-8971

0.746

v. 76 , fisrt page 161 , last page 171

0.746

0001-3765

0.737

v. 76 , fisrt page 429 , last page 434

0001-3765

0.737

10

10

10

6.9

6.9

6.9

9.4

4.5

3.2

10

4

4.8

10

8.2

8.2

8.2

8.2

8.2

8.5

8.5 
Journal

Anais da Academia Brasileira de Ciências

Anais da Academia Brasileira de Ciências

Anais da Academia Brasileira de Ciências

Anais da Academia Brasileira de Ciências

Anais da Academia Brasileira de Ciências

Anais da Academia Brasileira de Ciências

Anais da Academia Brasileira de Ciências

Anais da Academia Brasileira de Ciências

Anais da Academia Brasileira de Ciências

Anais da Academia Brasileira de Ciências

Anais da Academia Brasileira de Ciências

Anais da Academia Brasileira de Ciências

Anais da Academia Brasileira de Ciências

Journal of Micropalaeontology

Limnologica (Jena)

Cryptogamie. Bryologie

Cryptogamie. Bryologie

Química Nova

Química Nova

Química Nova

Química Nova

Química Nova

Química Nova

Química Nova

Química Nova

Química Nova

Química Nova

Química Nova

Química Nova

Química Nova

Química Nova

Química Nova

Química Nova

Química Nova

Química Nova

Química Nova

Zeitschrift für Naturforschung. C, A Journal of Biosciences

Zeitschrift für Naturforschung. C, A Journal of Biosciences

Zeitschrift für Naturforschung. C, A Journal of Biosciences

Zeitschrift für Naturforschung. C, A Journal of Biosciences

Journal of Horticultural Science and

Biotechnology

Experimental \& Applied Acarology

Experimental \& Applied Acarology

Zoosystema (Paris)

Zoosystema (Paris)

Zoosystema (Paris)

Zoosystema (Paris)

Zoosystema (Paris)

Wilderness \& Environmental Medicine

Wilderness \& Environmental Medicine

Wilderness \& Environmental Medicine

Scientia Horticulturae

Scientia Horticulturae

Scientia Horticulturae

Scientia Horticulturae

Grana (Stockholm)
Paper info.

v. 76, fisrt page 499, last page 504

v. 76 , fisrt page 625 , last page 630

v. 76 , fisrt page 651 , last page 661

v. 76 , fisrt page 85 , last page 92

v. 77 , fisrt page 103 , last page 111

v. 77 , fisrt page 259 , last page 274

v. 77 , fisrt page 343 , last page 352

v. 77 , fisrt page 549 , last page 579

v. 78 , fisrt page 175 , last page 182

v. 78, fisrt page 203 , last page 212

v. 78 , fisrt page 213 , last page 226

v. 78 , fist page 45 , last page 57

v. 78 , fist page 59 , last page 68

v. 78 , fisrt page 667 , last page 686

v. 23 , fisrt page 107 , last page 118

v. 36, fisrt page 192, last page 202

v. 26 , fisrt page 239 , last page 247

v. 27 , fisrt page 153 , last page 163

v. 27 , fisrt page 196, last page 198

v. 27 , fisrt page 414 , last page 416

v. 27 , fisrt page 46 , last page 49

v. 27 , fisrt page 536 , last page 539

v. 27 , fisrt page 55 , last page 57

v. 27 , fisrt page 763 , last page 770

v. 27 , fisrt page 878 , last page 881

v. 28 , fisrt page 192, last page 198

v. 28, fisrt page 199, last page 203

v. 28 , fisrt page 444 , last page 450

v. 28 , fisrt page 462 , last page 471

v. 28 , fisrt page 472 , last page 477

v. 28 , fisrt page 601 , last page 604

v. 28 , fisrt page 892 , last page 896

v. 29 , fisrt page 1 , last page 5

v. 29, fisrt page 1279, last page 1286

v. 29 , fisrt page 245 , last page 246

v. 29 , fisrt page 467 , last page 470

v. 29 , fisrt page 695 , last page 698

v. 60 c, fisrt page 394 , last page 398

v. 60 c, fisrt page 539 , last page 543

v. 61 , fisrt page 177 , last page 183

v. 61 , fisrt page 643 , last page 646

v. 81 , fisrt page 310 , last page 314

v. 32 , fist page 79 , last page 88

v. 38, fisrt page 242 , last page 273

v. 25 , fisrt page 43, last page 129

v. 26 , fisrt page 157 , last page 173

v. 27 , fisrt page 441 , last page 467

v. 27 , fisrt page 5 , last page 25

v. 28 , fisrt page 533 , last page 537

v. 16 , fisrt page 204 , last page 208

v. 16 , fist page 97 , last page 100

v. 80 , fisrt page 571 , last page 578

v. 100 , fisrt page 63 , last page 74

v. 107 , fisrt page 358 , last page 364

v. 109 , fisrt page 160 , last page 164

v. 99 , fisrt page 371 , last page 378

v. 44 , fisrt page 65 , last page 74
ISSN

0001-3765

IF Half-Life

0001-3765

0.737

8.5

0001-3765

0.737

0001-3765

0.737

0.737

$0001-3765$

0.737

0001-3765

0.737

0001-3765

0.737

0.737

0.737

0001-3765

0001-3765

0.737

0001-3765

0.737

0001-3765

0001-3765

0001-3765

0262-821X

$0075-9511$

1290-0796

1290-0796

0100-4042

0100-4042

0100-4042

0100-4042

0100-4042

0100-4042

0100-4042

0100-4042

0100-4042

0100-4042

0100-4042

0100-4042

0100-4042

0100-4042

0100-4042

0100-4042

0100-4042

0100-4042

0100-4042

0939-5075

0.737

0.737

0.737

0.722

0.722

0.721

0.721

0.72

0.72

0.72

0.72

0.72

0.72

0.72

0.72

0.72

0.72

0.72

0.72

0.72

0.72

0.72

0.72

0.72

0.72

0.72

0.72

0939-5075

0.72

0939-5075

0.72

0939-5075

0.72

0022-1589

0168-8162

0.719

0168-8162

0.716

0.716

1280-9551

1280-9551

1280-9551

1280-9551

1280-9551

1080-6032

1080-6032

1080-6032

0304-4238

0304-4238

0304-4238

0304-4238

0017-3134

0.714

0.714

0.714

0.714

0.714

0.698

0.698

0.698

0.697

0.697

0.697

0.697

0.689
8.9

8.9

8.9

8.9

9.6

7.5

8.5
8.5

8.5

8.5

8.5

8.5

8.5

8.5

8.5

8.5

8.5

8.5

8.5

10

6.8

6.8

5

5

5

5

5

5

5

5

5

5

5

5

5

5

5

5

5

5

7.5

5.3

5.3

5.3

5.3

5.3

5

5

5

7.8

7.8

7.8

7.8

10 


\begin{tabular}{|c|c|c|c|c|}
\hline $\begin{array}{r}\text { Journal } \\
\end{array}$ & Paper info. & ISSN & IF & Half-Life \\
\hline Building and Environment & v. 41 , fisrt page 1540 , last page 1543 & 0360-1323 & 0.686 & 5.2 \\
\hline Journal of the World Aquaculture Society & v. 36 , fisrt page 42 , last page 50 & 0893-8849 & 0.684 & 9.1 \\
\hline Annales de la Société Entomologique de & v. 42 , fisrt page 165 , last page 170 & 0037-9271 & & \\
\hline France & & & 0.683 & 10 \\
\hline Candollea (Genève) & v. 61 , fisrt page 453 , last page 456 & 0373-2967 & 0.674 & 10 \\
\hline Florida Entomologist & v. 87 , fisrt page 517 , last page 521 & $0015-4040$ & 0.667 & 10 \\
\hline IAWA Journal & v. 25 , fisrt page 175 , last page 183 & $0928-1541$ & 0.667 & 10 \\
\hline IAWA Journal & v. 26 , fisrt page 299 , last page 308 & $0928-1541$ & 0.667 & 10 \\
\hline IAWA Journal & v. 26 , fisrt page 477 , last page 487 & $0928-1541$ & 0.667 & 10 \\
\hline IAWA Journal & v. 26, fisrt page 93, last page 98 & $0928-1541$ & 0.667 & 10 \\
\hline IAWA Journal & v. 27 , fisrt page 199 , last page 211 & $0928-1541$ & 0.667 & 10 \\
\hline IAWA Journal & v. 27 , fisrt page 269 , last page 280 & $0928-1541$ & 0.667 & 10 \\
\hline IAWA Journal & v. 3, fisrt page 255 , last page 266 & 0928-1541 & 0.667 & 10 \\
\hline Journal of Coastal Research & v. 21 , fist page 1121 , last page 1126 & 0749-0208 & 0.665 & 8.3 \\
\hline Journal of Coastal Research & v. 21, fisrt page 18, last page 27 & 0749-0208 & 0.665 & 8.3 \\
\hline Journal of Coastal Research & v. 21 , fisrt page 751 , last page 758 & 0749-0208 & 0.665 & 8.3 \\
\hline Journal of Coastal Research & v. 21 , fisrt page 811 , last page 817 & 0749-0208 & 0.665 & 8.3 \\
\hline Journal of Coastal Research & v. 21, fisrt page 909, last page 914 & 0749-0208 & 0.665 & 8.3 \\
\hline Journal of Coastal Research & v. 22, fisrt page 1070, last page 1075 & 0749-0208 & 0.665 & 8.3 \\
\hline Journal of Coastal Research & v. 22 , fisrt page 825 , last page 835 & 0749-0208 & 0.665 & 8.3 \\
\hline Journal of Coastal Research & v. $39 \mathrm{SI}$, fistt page 125 , last page 131 & 0749-0208 & 0.665 & 8.3 \\
\hline Journal of Coastal Research & v. 39 , fisrt page 1135 , last page 1139 & 0749-0208 & 0.665 & 8.3 \\
\hline Journal of Coastal Research & v. 39 , fisrt page 1140 , last page 1143 & 0749-0208 & 0.665 & 8.3 \\
\hline Journal of Coastal Research & v. 39 , fisrt page 1161 , last page 1164 & 0749-0208 & 0.665 & 8.3 \\
\hline Journal of Coastal Research & v. 39 , fisrt page 1165 , last page 1168 & 0749-0208 & 0.665 & 8.3 \\
\hline Journal of Coastal Research & v. 39, fisrt page 1186, last page 1189 & 0749-0208 & 0.665 & 8.3 \\
\hline Journal of Coastal Research & v. 39, fisrt page 1295, last page 1299 & 0749-0208 & 0.665 & 8.3 \\
\hline Journal of Coastal Research & v. 39, fisrt page 1676, last page 1679 & 0749-0208 & 0.665 & 8.3 \\
\hline Journal of Coastal Research & v. 39, fisrt page 1695, last page 1699 & 0749-0208 & 0.665 & 8.3 \\
\hline Journal of Coastal Research & v. 39 , fisrt page 172 , last page 180 & 0749-0208 & 0.665 & 8.3 \\
\hline Journal of Coastal Research & v. 39 , fisrt page 1877 , last page 1881 & 0749-0208 & 0.665 & 8.3 \\
\hline Journal of Coastal Research & v. 39, fisrt page 235 , last page 245 & 0749-0208 & 0.665 & 8.3 \\
\hline Journal of Coastal Research & v. 39 , fisrt page 306 , last page 310 & 0749-0208 & 0.665 & 8.3 \\
\hline Journal of Coastal Research & v. 39, fisrt page 35 , last page 46 & 0749-0208 & 0.665 & 8.3 \\
\hline Journal of Coastal Research & v. 39, fisrt page 5 , last page 8 & 0749-0208 & 0.665 & 8.3 \\
\hline Journal of Coastal Research & v. 39 , fisrt page 622 , last page 625 & 0749-0208 & 0.665 & 8.3 \\
\hline Journal of Coastal Research & v. 39 , fisrt page 644 , last page 647 & 0749-0208 & 0.665 & 8.3 \\
\hline Journal of Coastal Research & v. 42, fisrt page 191, last page 197 & 0749-0208 & 0.665 & 8.3 \\
\hline Journal of Coastal Research & v. 42 , fisrt page 265 , last page 270 & 0749-0208 & 0.665 & 8.3 \\
\hline Journal of Coastal Research & v. 47 , fisrt page 1871 , last page 1876 & 0749-0208 & 0.665 & 8.3 \\
\hline Journal of Coastal Research & v. S139, fistt page 129 , last page 229 & 0749-0208 & 0.665 & 8.3 \\
\hline Journal of Coastal Research & v. SI 35 , fisrt page 426 , last page 430 & 0749-0208 & 0.665 & 8.3 \\
\hline Journal of Coastal Research & v. SI 39, fisrt page 1036, last page 1039 & 0749-0208 & 0.665 & 8.3 \\
\hline Journal of Coastal Research & v. SI 39, fisrt page 1040, last page 1043 & 0749-0208 & 0.665 & 8.3 \\
\hline Journal of Coastal Research & v. SI 39, fisrt page 1098, last page 1101 & 0749-0208 & 0.665 & 8.3 \\
\hline Journal of Coastal Research & v. SI 39, fisrt page 11, last page 20 & 0749-0208 & 0.665 & 8.3 \\
\hline Journal of Coastal Research & v. SI 39, fisrt page 1149, last page 1151 & 0749-0208 & 0.665 & 8.3 \\
\hline Journal of Coastal Research & v. SI 39, fisrt page 1246 , last page 1250 & 0749-0208 & 0.665 & 8.3 \\
\hline Journal of Coastal Research & v. SI 39, fisrt page 1357, last page 1359 & 0749-0208 & 0.665 & 8.3 \\
\hline Journal of Coastal Research & v. SI 39, fisrt page 15 , last page 24 & 0749-0208 & 0.665 & 8.3 \\
\hline Journal of Coastal Research & v. SI 39, fisrt page 1546 , last page 1550 & 0749-0208 & 0.665 & 8.3 \\
\hline Journal of Coastal Research. & v. 39, fisrt page 876, last page 987 & 0749-0208 & 0.665 & 8.3 \\
\hline $\begin{array}{l}\text { Journal of Drug Delivery Science and } \\
\text { Technology }\end{array}$ & v. 15 , fisrt page 355 , last page 361 & 1773-2247 & 0.664 & \\
\hline $\begin{array}{l}\text { Studies on Neotropical Fauna and } \\
\text { Environment }\end{array}$ & v. 1 , fistt page 109 , last page 116 & $0165-0521$ & 0661 & 10 \\
\hline Studies on Neotropical Fauna and & v. 128 , fist page 204 , last page 211 & $0165-0521$ & & \\
\hline Environment & & & 0.661 & 10 \\
\hline $\begin{array}{l}\text { Studies on Neotropical Fauna and } \\
\text { Environment }\end{array}$ & v. 39 , fisrt page 109 , last page 116 & 0165-0521 & 0.661 & 10 \\
\hline Studies on Neotropical Fauna and & v. 39, fisrt page 191, last page 199 & $0165-0521$ & & \\
\hline Environment & & & 0.661 & 10 \\
\hline
\end{tabular}




\begin{tabular}{|c|c|c|c|c|}
\hline$\frac{\text { Journal }}{\text { Studipal }}$ & $\begin{array}{c}\text { Paper info. } \\
\text { y } 39 \text { fist nage } 53 \text { last nage } 56\end{array}$ & $\frac{\text { ISSN }}{0165-0521}$ & IF & Half-Life \\
\hline $\begin{array}{l}\text { Studies on Neotropical Fauna and } \\
\text { Environment }\end{array}$ & v. 39 , fisrt page 53 , last page 56 & 0165-0521 & 0.661 & 10 \\
\hline Studies on Neotropical Fauna and & v. 39 , fisrt page 57 , last page 61 & 0165-0521 & & \\
\hline $\begin{array}{l}\text { Environment } \\
\text { Studies on Neotropical Fauna and }\end{array}$ & v. 39, fistt page 7 , last page 14 & $0165-0521$ & 0.661 & 10 \\
\hline Environment & & & 0.661 & 10 \\
\hline Studies on Neotropical Fauna and & v. 4 , fisrt page 49 , last page 60 & 0165-0521 & & \\
\hline $\begin{array}{l}\text { Environment } \\
\text { Studies on Neotropical Fauna and }\end{array}$ & v. 40 , fisrt page 143 , last page 148 & $0165-0521$ & 0.661 & 10 \\
\hline Environment & & & 0.661 & 10 \\
\hline $\begin{array}{l}\text { Studies on Neotropical Fauna and } \\
\text { Environment }\end{array}$ & v. 40 , fisrt page 187 , last page 190 & 0165-0521 & 0.661 & 10 \\
\hline Studies on Neotropical Fauna and & v. 41 , fisrt page 227 , last page 233 & 0165-0521 & & \\
\hline $\begin{array}{l}\text { Environment } \\
\text { Studies on Neotropical Fauna and }\end{array}$ & v 41 fist page 9 Jast page 17 & $0165-0521$ & 0.661 & 10 \\
\hline $\begin{array}{l}\text { Environment } \\
\text { Entropical rauna and }\end{array}$ & v. 41, fisrt page 9, last page 17 & $0165-0521$ & 0.661 & 10 \\
\hline Studies on Neotropical Fauna and & v. 42 , fisrt page 1 , last page 10 & 0165-0521 & & \\
\hline $\begin{array}{l}\text { Environment } \\
\text { Studies }\end{array}$ & & & 0.661 & 10 \\
\hline $\begin{array}{l}\text { Studies on Neotropical Fauna and } \\
\text { Environment }\end{array}$ & v. 42 , fisrt page 123 , last page 129 & $0165-0521$ & 0.661 & 10 \\
\hline Journal of Field Ornithology & v. 76 , fisrt page 235 , last page 237 & 0273-8570 & 0.66 & 10 \\
\hline Comparative Parasitology & v. 71 , fisrt page 1 , last page 3 & $1525-2647$ & 0.659 & 4 \\
\hline Comparative Parasitology & v. 73 , fisrt page 258 , last page 263 & $1525-2647$ & 0.659 & 4 \\
\hline Insect Systematics \& Evolution & v. 35 , fisrt page 189 , last page 240 & $1399-560 X$ & 0.649 & 3.9 \\
\hline Insect Systematics \& Evolution & v. 36 , fisrt page 285 , last page 292 & $1399-560 X$ & 0.649 & 3.9 \\
\hline Insect Systematics \& Evolution & v. 36, fisrt page 35 , last page 56 & $1399-560 X$ & 0.649 & 3.9 \\
\hline Revista Chilena de Historia Natural & v. 77 , fisrt page 285 , last page 292 & 0716-078X & 0.646 & 8.1 \\
\hline Revista Chilena de Historia Natural & v. 77 , fisrt page 485 , last page 490 & 0716-078X & 0.646 & 8.1 \\
\hline Revista Chilena de Historia Natural & v. 77 , fisrt page 5 , last page 14 & 0716-078X & 0.646 & 8.1 \\
\hline Journal of Natural History & v. 24, fisrt page 901 , last page 912 & 0022-2933 & 0.631 & 10 \\
\hline Journal of Natural History & v. 28 , fisrt page 1605 , last page 1634 & 0022-2933 & 0.631 & 10 \\
\hline Journal of Natural History & v. 36 , fisrt page 3275 , last page 3295 & 0022-2933 & 0.631 & 10 \\
\hline Journal of Natural History & v. 38, fisrt page 1071, last page 1080 & $0022-2933$ & 0.631 & 10 \\
\hline Journal of Natural History & v. 38, fisrt page 1443, last page 1455 & $0022-2933$ & 0.631 & 10 \\
\hline Journal of Natural History & v. 38, fisrt page 1695 , last page 1710 & $0022-2933$ & 0.631 & 10 \\
\hline Journal of Natural History & v. 38, fisrt page 1773, last page 1997 & 0022-2933 & 0.631 & 10 \\
\hline Journal of Natural History & v. 38, fisrt page 2073, last page 2083 & 0022-2933 & 0.631 & 10 \\
\hline Journal of Natural History & v. 38, fisrt page 2331 , last page 2344 & 0022-2933 & 0.631 & 10 \\
\hline Journal of Natural History & v. 38 , fisrt page 2361 , last page 2375 & 0022-2933 & 0.631 & 10 \\
\hline Journal of Natural History & v. 38 , fisrt page 2533 , last page 2546 & 0022-2933 & 0.631 & 10 \\
\hline Journal of Natural History & v. 38 , fisrt page 2567 , last page 2579 & 0022-2933 & 0.631 & 10 \\
\hline Journal of Natural History & v. 38 , fisrt page 2851 , last page 2862 & $0022-2933$ & 0.631 & 10 \\
\hline Journal of Natural History & v. 38, fisrt page 951 , last page 965 & 0022-2933 & 0.631 & 10 \\
\hline Journal of Natural History & v. 39, fisrt page 1627, last page 1640 & $0022-2933$ & 0.631 & 10 \\
\hline Journal of Natural History & v. 39, fisrt page 1675, last page 1685 & $0022-2933$ & 0.631 & 10 \\
\hline Journal of Natural History & v. 39, fisrt page 1851, last page 1873 & 0022-2933 & 0.631 & 10 \\
\hline Journal of Natural History & v. 39, fisrt page 2035, last page 2045 & $0022-2933$ & 0.631 & 10 \\
\hline Journal of Natural History & v. 39, fisrt page 2145 , last page 2161 & 0022-2933 & 0.631 & 10 \\
\hline Journal of Natural History & v. 39, fisrt page 3029 , last page 3037 & $0022-2933$ & 0.631 & 10 \\
\hline Journal of Natural History & v. 39, fisrt page 709 , last page 722 & $0022-2933$ & 0.631 & 10 \\
\hline Journal of Natural History & v. 40, fisrt page 1047, last page 1062 & $0022-2933$ & 0.631 & 10 \\
\hline Journal of Natural History & v. 40, fisrt page 1199, last page 1209 & $0022-2933$ & 0.631 & 10 \\
\hline Journal of Natural History & v. 40 , fisrt page 126 , last page 130 & 0022-2933 & 0.631 & 10 \\
\hline Journal of Natural History & v. 40 , fisrt page 1831 , last page 1840 & 0022-2933 & 0.631 & 10 \\
\hline Journal of Natural History & v. 40 , fisrt page 915 , last page 930 & 0022-2933 & 0.631 & 10 \\
\hline Journal of Natural History & v. 40, fisrt page 999, last page 1005 & 0022-2933 & 0.631 & 10 \\
\hline Journal of Veterinary Medicine. Series A & v. A52, fisrt page 359, last page 365 & 0931-184X & 0.627 & 6.5 \\
\hline African Entomology & v. 12 , fisrt page 157, last page 163 & 1021-3589 & 0.613 & 6 \\
\hline African Entomology & v. 12, fisrt page 97 , last page 105 & 1021-3589 & 0.613 & 6 \\
\hline Zootaxa (Auckland) & v. 1006 , fisrt page 11 , last page 21 & $1175-5326$ & 0.612 & 2 \\
\hline Zootaxa (Auckland) & v. 1006 , fisrt page 23 , last page 32 & $1175-5326$ & 0.612 & 2 \\
\hline Zootaxa (Auckland) & v. 1006 , fisrt page 43 , last page 52 & $1175-5326$ & 0.612 & 2 \\
\hline Zootaxa (Auckland) & v. 1012, fisrt page 13, last page 22 & $1175-5326$ & 0.612 & 2 \\
\hline Zootaxa (Auckland) & v. 1013 , fisrt page 19 , last page 34 & $1175-5326$ & 0.612 & 2 \\
\hline
\end{tabular}




\begin{tabular}{|c|c|c|c|c|}
\hline Journal & Paper info. & ISSN & IF & Half-Life \\
\hline Zootaxa (Auckland) & v. 1016 , fisrt page 39 , last page 47 & $1175-5326$ & 0.612 & 2 \\
\hline Zootaxa (Auckland) & v. 1016 , fisrt page 49 , last page 55 & $1175-5326$ & 0.612 & 2 \\
\hline Zootaxa (Auckland) & v. 1018 , fisrt page 55 , last page 60 & $1175-5326$ & 0.612 & 2 \\
\hline Zootaxa (Auckland) & v. 1021 , fisrt page 37 , last page 43 & $1175-5326$ & 0.612 & 2 \\
\hline Zootaxa (Auckland) & v. 1023 , fisrt page 1 , last page 760 & $1175-5326$ & 0.612 & 2 \\
\hline Zootaxa (Auckland) & v. 1031 , fisrt page 1 , last page 76 & $1175-5326$ & 0.612 & 2 \\
\hline Zootaxa (Auckland) & v. 1036 , fistt page 31 , last page 42 & $1175-5326$ & 0.612 & 2 \\
\hline Zootaxa (Auckland) & v. 1039 , fistt page 19 , last page 26 & $1175-5326$ & 0.612 & 2 \\
\hline Zootaxa (Auckland) & v. 1039 , fistt page 39 , last page 55 & $1175-5326$ & 0.612 & 2 \\
\hline Zootaxa (Auckland) & v. 1039 , fisrt page 57 , last page 64 & $1175-5326$ & 0.612 & 2 \\
\hline Zootaxa (Auckland) & v. 1040 , fistt page 49 , last page 64 & $1175-5326$ & 0.612 & 2 \\
\hline Zootaxa (Auckland) & v. 1046 , fisrt page 37 , last page 47 & $1175-5326$ & 0.612 & 2 \\
\hline Zootaxa (Auckland) & v. 1049 , fisrt page 49 , last page 68 & $1175-5326$ & 0.612 & 2 \\
\hline Zootaxa (Auckland) & v. 1052 , fistt page 41 , last page 48 & $1175-5326$ & 0.612 & 2 \\
\hline Zootaxa (Auckland) & v. 1053, fisrt page 1 , last page 21 & $1175-5326$ & 0.612 & 2 \\
\hline Zootaxa (Auckland) & v. 1054 , fisrt page 25 , last page 29 & $1175-5326$ & 0.612 & 2 \\
\hline Zootaxa (Auckland) & v. 1060 , fistt page 27 , last page 36 & $1175-5326$ & 0.612 & 2 \\
\hline Zootaxa (Auckland) & v. 1064 , fistt page 25 , last page 30 & $1175-5326$ & 0.612 & 2 \\
\hline Zootaxa (Auckland) & v. 1066 , fistt page 43 , last page 56 & $1175-5326$ & 0.612 & 2 \\
\hline Zootaxa (Auckland) & v. 1067 , fisrt page 1 , last page 36 & $1175-5326$ & 0.612 & 2 \\
\hline Zootaxa (Auckland) & v. 1072 , fistt page 35 , last page 42 & $1175-5326$ & 0.612 & 2 \\
\hline Zootaxa (Auckland) & v. 1081, fistt page 1 , last page 31 & $1175-5326$ & 0.612 & 2 \\
\hline Zootaxa (Auckland) & v. 1081 , fistt page 33 , last page 45 & $1175-5326$ & 0.612 & 2 \\
\hline Zootaxa (Auckland) & v. 1081 , fistt page 47 , last page 59 & $1175-5326$ & 0.612 & 2 \\
\hline Zootaxa (Auckland) & v. 1084 , fisrt page 31 , last page 42 & $1175-5326$ & 0.612 & 2 \\
\hline Zootaxa (Auckland) & v. 1087 , fisrt page 39 , last page 44 & $1175-5326$ & 0.612 & 2 \\
\hline Zootaxa (Auckland) & v. 1087 , fisrt page 45 , last page 52 & $1175-5326$ & 0.612 & 2 \\
\hline Zootaxa (Auckland) & v. 1088 , fisrt page 1 , last page 10 & $1175-5326$ & 0.612 & 2 \\
\hline Zootaxa (Auckland) & v. 1088 , fistt page 11 , last page 16 & $1175-5326$ & 0.612 & 2 \\
\hline Zootaxa (Auckland) & v. 1088 , fistt page 55 , last page 59 & $1175-5326$ & 0.612 & 2 \\
\hline Zootaxa (Auckland) & v. 1090 , fisrt page 51 , last page 64 & $1175-5326$ & 0.612 & 2 \\
\hline Zootaxa (Auckland) & v. 1091 , fistt page 27 , last page 40 & $1175-5326$ & 0.612 & 2 \\
\hline Zootaxa (Auckland) & v. 1105 , fisrt page 27 , last page 35 & $1175-5326$ & 0.612 & 2 \\
\hline Zootaxa (Auckland) & v. 1109 , fistt page 15 , last page 26 & $1175-5326$ & 0.612 & 2 \\
\hline Zootaxa (Auckland) & v. 1112, fisrt page 1 , last page 64 & $1175-5326$ & 0.612 & 2 \\
\hline Zootaxa (Auckland) & v. 1119 , fisrt page 29 , last page 58 & $1175-5326$ & 0.612 & 2 \\
\hline Zootaxa (Auckland) & v. 1120 , fisrt page 56 , last page 68 & $1175-5326$ & 0.612 & 2 \\
\hline Zootaxa (Auckland) & v. 1121 , fistt page 53 , last page 68 & $1175-5326$ & 0.612 & 2 \\
\hline Zootaxa (Auckland) & v. 1123, fistt page 1 , last page 19 & $1175-5326$ & 0.612 & 2 \\
\hline Zootaxa (Auckland) & v. 1124 , fisrt page 1 , last page 40 & $1175-5326$ & 0.612 & 2 \\
\hline Zootaxa (Auckland) & v. 1126 , fisrt page 63 , last page 68 & $1175-5326$ & 0.612 & 2 \\
\hline Zootaxa (Auckland) & v. 1135 , fisrt page 29 , last page 48 & $1175-5326$ & 0.612 & 2 \\
\hline Zootaxa (Auckland) & v. 1136 , fisrt page 39 , last page 48 & $1175-5326$ & 0.612 & 2 \\
\hline Zootaxa (Auckland) & v. 1142 , fistt page 43 , last page 50 & $1175-5326$ & 0.612 & 2 \\
\hline Zootaxa (Auckland) & v. 1151 , fistt page 1 , last page 26 & $1175-5326$ & 0.612 & 2 \\
\hline Zootaxa (Auckland) & v. 1155 , fistt page 61 , last page 68 & $1175-5326$ & 0.612 & 2 \\
\hline Zootaxa (Auckland) & v. 1174 , fistt page 27 , last page 39 & $1175-5326$ & 0.612 & 2 \\
\hline Zootaxa (Auckland) & v. 1177 , fisrt page 21 , last page 26 & $1175-5326$ & 0.612 & 2 \\
\hline Zootaxa (Auckland) & v. 1183, fistt page 1 , last page 26 & $1175-5326$ & 0.612 & 2 \\
\hline Zootaxa (Auckland) & v. 1184 , fisrt page 29 , last page 42 & $1175-5326$ & 0.612 & 2 \\
\hline Zootaxa (Auckland) & v. 1185 , fistt page 21 , last page 35 & $1175-5326$ & 0.612 & 2 \\
\hline Zootaxa (Auckland) & v. 1191 , fistt page 35 , last page 47 & $1175-5326$ & 0.612 & 2 \\
\hline Zootaxa (Auckland) & v. 1195 , fistt page 31 , last page 37 & $1175-5326$ & 0.612 & 2 \\
\hline Zootaxa (Auckland) & v. 1199 , fistt page 61 , last page 68 & $1175-5326$ & 0.612 & 2 \\
\hline Zootaxa (Auckland) & v. 1200 , fisrt page 1 , last page 12 & $1175-5326$ & 0.612 & 2 \\
\hline Zootaxa (Auckland) & v. 1200 , fisrt page 43 , last page 60 & $1175-5326$ & 0.612 & 2 \\
\hline Zootaxa (Auckland) & v. 1205 , fistt page 31 , last page 54 & $1175-5326$ & 0.612 & 2 \\
\hline Zootaxa (Auckland) & v. 1212 , fistt page 1 , last page 244 & $1175-5326$ & 0.612 & 2 \\
\hline Zootaxa (Auckland) & v. 1213 , fistt page 1 , last page 162 & $1175-5326$ & 0.612 & 2 \\
\hline Zootaxa (Auckland) & v. 1221 , fistt page 41 , last page 62 & $1175-5326$ & 0.612 & 2 \\
\hline
\end{tabular}




\begin{tabular}{|c|c|c|c|c|}
\hline Journal & Paper info. & ISSN & IF & Half-Life \\
\hline Zootaxa (Auckland) & v. 1227 , fisrt page 31 , last page 55 & $1175-5326$ & 0.612 & 2 \\
\hline Zootaxa (Auckland) & v. 1236 , fisrt page 37 , last page 52 & $1175-5326$ & 0.612 & 2 \\
\hline Zootaxa (Auckland) & v. 1237 , fisrt page 19 , last page 25 & $1175-5326$ & 0.612 & 2 \\
\hline Zootaxa (Auckland) & v. 1242 , fistt page 37 , last page 52 & $1175-5326$ & 0.612 & 2 \\
\hline Zootaxa (Auckland) & v. 1244 , fisrt page 41 , last page 55 & $1175-5326$ & 0.612 & 2 \\
\hline Zootaxa (Auckland) & v. 1247 , fisrt page 1 , last page 12 & $1175-5326$ & 0.612 & 2 \\
\hline Zootaxa (Auckland) & v. 1249 , fisrt page 23 , last page 36 & $1175-5326$ & 0.612 & 2 \\
\hline Zootaxa (Auckland) & v. 1256 , fisrt page 21 , last page 47 & $1175-5326$ & 0.612 & 2 \\
\hline Zootaxa (Auckland) & v. 1269 , fistt page 43 , last page 49 & $1175-5326$ & 0.612 & 2 \\
\hline Zootaxa (Auckland) & v. 1269 , fistt page 51 , last page 56 & $1175-5326$ & 0.612 & 2 \\
\hline Zootaxa (Auckland) & v. 1271 , fistt page 37 , last page 56 & $1175-5326$ & 0.612 & 2 \\
\hline Zootaxa (Auckland) & v. 1275 , fisrt page 61 , last page 68 & $1175-5326$ & 0.612 & 2 \\
\hline Zootaxa (Auckland) & v. 1277 , fistt page 23 , last page 27 & $1175-5326$ & 0.612 & 2 \\
\hline Zootaxa (Auckland) & v. 1285 , fistt page 21 , last page 29 & $1175-5326$ & 0.612 & 2 \\
\hline Zootaxa (Auckland) & v. 1296 , fistt page 19 , last page 28 & $1175-5326$ & 0.612 & 2 \\
\hline Zootaxa (Auckland) & v. 1298 , fistt page 49 , last page 60 & $1175-5326$ & 0.612 & 2 \\
\hline Zootaxa (Auckland) & v. 1300, fisrt page 1 , last page 29 & $1175-5326$ & 0.612 & 2 \\
\hline Zootaxa (Auckland) & v. 1300 , fistt page 37 , last page 50 & $1175-5326$ & 0.612 & 2 \\
\hline Zootaxa (Auckland) & v. 1306 , fisrt page 25 , last page 39 & $1175-5326$ & 0.612 & 2 \\
\hline Zootaxa (Auckland) & v. 1314 , fisrt page 37 , last page 50 & $1175-5326$ & 0.612 & 2 \\
\hline Zootaxa (Auckland) & v. 1314 , fisrt page 53 , last page 68 & $1175-5326$ & 0.612 & 2 \\
\hline Zootaxa (Auckland) & v. 1318 , fisrt page 1 , last page 40 & $1175-5326$ & 0.612 & 2 \\
\hline Zootaxa (Auckland) & v. 1320 , fistt page 57 , last page 68 & $1175-5326$ & 0.612 & 2 \\
\hline Zootaxa (Auckland) & v. 1321 , fisrt page 1 , last page 108 & $1175-5326$ & 0.612 & 2 \\
\hline Zootaxa (Auckland) & v. 1325 , fisrt page 219 , last page 233 & $1175-5326$ & 0.612 & 2 \\
\hline Zootaxa (Auckland) & v. 1329 , fisrt page 59 , last page 68 & $1175-5326$ & 0.612 & 2 \\
\hline Zootaxa (Auckland) & v. 1330, fisrt page 1 , last page 26 & $1175-5326$ & 0.612 & 2 \\
\hline Zootaxa (Auckland) & v. 1330 , fistt page 43 , last page 50 & $1175-5326$ & 0.612 & 2 \\
\hline Zootaxa (Auckland) & v. 1331 , fisrt page 1 , last page 30 & $1175-5326$ & 0.612 & 2 \\
\hline Zootaxa (Auckland) & v. 1335 , fisrt page 1 , last page 50 & $1175-5326$ & 0.612 & 2 \\
\hline Zootaxa (Auckland) & v. 1340 , fisrt page 1 , last page 44 & $1175-5326$ & 0.612 & 2 \\
\hline Zootaxa (Auckland) & v. 1350 , fisrt page 21 , last page 31 & $1175-5326$ & 0.612 & 2 \\
\hline Zootaxa (Auckland) & v. 1360 , fistt page 51 , last page 60 & $1175-5326$ & 0.612 & 2 \\
\hline Zootaxa (Auckland) & v. 1361 , fistt page 45 , last page 51 & $1175-5326$ & 0.612 & 2 \\
\hline Zootaxa (Auckland) & v. 1362 , fistt page 43 , last page 53 & $1175-5326$ & 0.612 & 2 \\
\hline Zootaxa (Auckland) & v. 1364 , fisrt page 1 , last page 44 & $1175-5326$ & 0.612 & 2 \\
\hline Zootaxa (Auckland) & v. 1365 , fisrt page 37 , last page 47 & $1175-5326$ & 0.612 & 2 \\
\hline Zootaxa (Auckland) & v. 1365 , fistt page 61 , last page 68 & $1175-5326$ & 0.612 & 2 \\
\hline Zootaxa (Auckland) & v. 1366 , fistt page 45 , last page 54 & $1175-5326$ & 0.612 & 2 \\
\hline Zootaxa (Auckland) & v. 1368 , fisrt page 49 , last page 56 & $1175-5326$ & 0.612 & 2 \\
\hline Zootaxa (Auckland) & v. 1369 , fistt page 43 , last page 53 & $1175-5326$ & 0.612 & 2 \\
\hline Zootaxa (Auckland) & v. 1372 , fistt page 53 , last page 68 & $1175-5326$ & 0.612 & 2 \\
\hline Zootaxa (Auckland) & v. 1383 , fistt page 57 , last page 68 & $1175-5326$ & 0.612 & 2 \\
\hline Zootaxa (Auckland) & v. 166 , fisrt page 49 , last page 55 & $1175-5326$ & 0.612 & 2 \\
\hline Zootaxa (Auckland) & v. 2004, fistt page 1, last page 6 & $1175-5326$ & 0.612 & 2 \\
\hline Zootaxa (Auckland) & v. 400 , fisrt page 1 , last page 6 & $1175-5326$ & 0.612 & 2 \\
\hline Zootaxa (Auckland) & v. 404, fisrt page 1, last page 7 & $1175-5326$ & 0.612 & 2 \\
\hline Zootaxa (Auckland) & v. 430 , fisrt page 1 , last page 26 & $1175-5326$ & 0.612 & 2 \\
\hline Zootaxa (Auckland) & v. 443, fisrt page 1 , last page 8 & $1175-5326$ & 0.612 & 2 \\
\hline Zootaxa (Auckland) & v. 464 , fisrt page 1 , last page 7 & $1175-5326$ & 0.612 & 2 \\
\hline Zootaxa (Auckland) & v. 468 , fisrt page 1 , last page 7 & $1175-5326$ & 0.612 & 2 \\
\hline Zootaxa (Auckland) & v. 479 , fisrt page 1 , last page 12 & $1175-5326$ & 0.612 & 2 \\
\hline Zootaxa (Auckland) & v. 490 , fisrt page 1 , last page 8 & $1175-5326$ & 0.612 & 2 \\
\hline Zootaxa (Auckland) & v. 492 , fisrt page 1 , last page 12 & $1175-5326$ & 0.612 & 2 \\
\hline Zootaxa (Auckland) & v. 504 , fisrt page 1 , last page 10 & $1175-5326$ & 0.612 & 2 \\
\hline Zootaxa (Auckland) & v. 505 , fisrt page 1 , last page 26 & $1175-5326$ & 0.612 & 2 \\
\hline Zootaxa (Auckland) & v. 529 , fisrt page 1 , last page 18 & $1175-5326$ & 0.612 & 2 \\
\hline Zootaxa (Auckland) & v. 530 , fisrt page 1 , last page 7 & $1175-5326$ & 0.612 & 2 \\
\hline Zootaxa (Auckland) & v. 540 , fisrt page 1 , last page 7 & $1175-5326$ & 0.612 & 2 \\
\hline Zootaxa (Auckland) & v. 544 , fisrt page 1 , last page 10 & $1175-5326$ & 0.612 & 2 \\
\hline
\end{tabular}




\begin{tabular}{|c|c|c|c|c|}
\hline Journal & Paper info. & ISSN & IF & Half-Life \\
\hline Zootaxa (Auckland) & v. 556 , fisrt page 1 , last page 10 & $1175-5326$ & 0.612 & 2 \\
\hline Zootaxa (Auckland) & v. 557 , fisrt page 1 , last page 16 & $1175-5326$ & 0.612 & 2 \\
\hline Zootaxa (Auckland) & v. 563 , fisrt page 1 , last page 10 & $1175-5326$ & 0.612 & 2 \\
\hline Zootaxa (Auckland) & v. 587 , fisrt page 1 , last page 11 & $1175-5326$ & 0.612 & 2 \\
\hline Zootaxa (Auckland) & v. 592, fisrt page 1 , last page 12 & $1175-5326$ & 0.612 & 2 \\
\hline Zootaxa (Auckland) & v. 598 , fisrt page 1 , last page 5 & $1175-5326$ & 0.612 & 2 \\
\hline Zootaxa (Auckland) & v. 603 , fisrt page 1 , last page 12 & $1175-5326$ & 0.612 & 2 \\
\hline Zootaxa (Auckland) & v. 612 , fisrt page 1 , last page 6 & $1175-5326$ & 0.612 & 2 \\
\hline Zootaxa (Auckland) & v. 621 , fisrt page 1 , last page 16 & $1175-5326$ & 0.612 & 2 \\
\hline Zootaxa (Auckland) & v. 640 , fisrt page 1 , last page 6 & $1175-5326$ & 0.612 & 2 \\
\hline Zootaxa (Auckland) & v. 642 , fisrt page 1 , last page 10 & $1175-5326$ & 0.612 & 2 \\
\hline Zootaxa (Auckland) & v. 645 , fisrt page 1 , last page 15 & $1175-5326$ & 0.612 & 2 \\
\hline Zootaxa (Auckland) & v. 651 , fisrt page 1 , last page 15 & $1175-5326$ & 0.612 & 2 \\
\hline Zootaxa (Auckland) & v. 656 , fisrt page 1 , last page 5 & $1175-5326$ & 0.612 & 2 \\
\hline Zootaxa (Auckland) & v. 661 , fist page 1 , last page 22 & $1175-5326$ & 0.612 & 2 \\
\hline Zootaxa (Auckland) & v. 665 , fisrt page 1 , last page 8 & $1175-5326$ & 0.612 & 2 \\
\hline Zootaxa (Auckland) & v. 666 , fist page 1 , last page 11 & $1175-5326$ & 0.612 & 2 \\
\hline Zootaxa (Auckland) & v. 675 , fisrt page 1 , last page 6 & $1175-5326$ & 0.612 & 2 \\
\hline Zootaxa (Auckland) & v. 677 , fisrt page 1 , last page 6 & $1175-5326$ & 0.612 & 2 \\
\hline Zootaxa (Auckland) & v. 678 , fisrt page 1 , last page 23 & $1175-5326$ & 0.612 & 2 \\
\hline Zootaxa (Auckland) & v. 679 , fisrt page 1 , last page 16 & $1175-5326$ & 0.612 & 2 \\
\hline Zootaxa (Auckland) & v. 687 , fisrt page 1 , last page 10 & $1175-5326$ & 0.612 & 2 \\
\hline Zootaxa (Auckland) & v. 688 , fisrt page 1 , last page 15 & $1175-5326$ & 0.612 & 2 \\
\hline Zootaxa (Auckland) & v. 690 , fisrt page 1 , last page 24 & $1175-5326$ & 0.612 & 2 \\
\hline Zootaxa (Auckland) & v. 693, fisrt page 1 , last page 8 & $1175-5326$ & 0.612 & 2 \\
\hline Zootaxa (Auckland) & v. 696 , fisrt page 1 , last page 8 & $1175-5326$ & 0.612 & 2 \\
\hline Zootaxa (Auckland) & v. 697, fisrt page 1 , last page 5 & $1175-5326$ & 0.612 & 2 \\
\hline Zootaxa (Auckland) & v. 707 , fisrt page 1 , last page 12 & $1175-5326$ & 0.612 & 2 \\
\hline Zootaxa (Auckland) & v. 710 , fisrt page 1 , last page 8 & $1175-5326$ & 0.612 & 2 \\
\hline Zootaxa (Auckland) & v. 712 , fisrt page 1 , last page 12 & $1175-5326$ & 0.612 & 2 \\
\hline Zootaxa (Auckland) & v. 718 , fisrt page 1 , last page 19 & $1175-5326$ & 0.612 & 2 \\
\hline Zootaxa (Auckland) & v. 737 , fist page 1 , last page 7 & $1175-5326$ & 0.612 & 2 \\
\hline Zootaxa (Auckland) & v. 744 , fisrt page 1 , last page 8 & $1175-5326$ & 0.612 & 2 \\
\hline Zootaxa (Auckland) & v. 761 , fist page 1 , last page 6 & $1175-5326$ & 0.612 & 2 \\
\hline Zootaxa (Auckland) & v. 789 , fist page 1 , last page 26 & $1175-5326$ & 0.612 & 2 \\
\hline Zootaxa (Auckland) & v. 795 , fistt page 1 , last page 6 & $1175-5326$ & 0.612 & 2 \\
\hline Zootaxa (Auckland) & v. 823 , fistt page 1 , last page 27 & $1175-5326$ & 0.612 & 2 \\
\hline Zootaxa (Auckland) & v. 835 , fisrt page 1 , last page 8 & $1175-5326$ & 0.612 & 2 \\
\hline Zootaxa (Auckland) & v. 836 , fisrt page 1 , last page 7 & $1175-5326$ & 0.612 & 2 \\
\hline Zootaxa (Auckland) & v. 844 , fist page 1 , last page 12 & $1175-5326$ & 0.612 & 2 \\
\hline Zootaxa (Auckland) & v. 846 , fist page 1 , last page 31 & $1175-5326$ & 0.612 & 2 \\
\hline Zootaxa (Auckland) & v. 848, fisrt page 1 , last page 9 & $1175-5326$ & 0.612 & 2 \\
\hline Zootaxa (Auckland) & v. 860 , fistt page 1 , last page 282 & $1175-5326$ & 0.612 & 2 \\
\hline Zootaxa (Auckland) & v. 890 , fistt page 1 , last page 12 & $1175-5326$ & 0.612 & 2 \\
\hline Zootaxa (Auckland) & v. 919 , fisrt page 1 , last page 16 & $1175-5326$ & 0.612 & 2 \\
\hline Zootaxa (Auckland) & v. 922 , fisrt page 1 , last page 12 & $1175-5326$ & 0.612 & 2 \\
\hline Zootaxa (Auckland) & v. 937, fisrt page 1, last page 12 & $1175-5326$ & 0.612 & 2 \\
\hline Zootaxa (Auckland) & v. 946, fisrt page 1 , last page 765 & $1175-5326$ & 0.612 & 2 \\
\hline Zootaxa (Auckland) & v. 962, fisrt page 1 , last page 6 & $1175-5326$ & 0.612 & 2 \\
\hline Zootaxa (Auckland) & v. 963 , fisrt page 1 , last page 22 & $1175-5326$ & 0.612 & 2 \\
\hline Zootaxa (Auckland) & v. 979 , fisrt page 1 , last page 12 & $1175-5326$ & 0.612 & 2 \\
\hline Zootaxa (Auckland) & v. 984 , fisrt page 1 , last page 8 & $1175-5326$ & 0.612 & 2 \\
\hline Zootaxa (Online) & v. 1 , fisrt page 61 , last page 67 & $1175-5334$ & 0.612 & 2 \\
\hline Zootaxa (Online) & v. 1032, fisrt page 31 , last page 42 & $1175-5334$ & 0.612 & 2 \\
\hline Zootaxa (Online) & v. 1040 , fisrt page 17 , last page 29 & $1175-5334$ & 0.612 & 2 \\
\hline Zootaxa (Online) & v. 1048 , fisrt page 27 , last page 44 & $1175-5334$ & 0.612 & 2 \\
\hline Zootaxa (Online) & v. 1051 , fisrt page 1 , last page 18 & $1175-5334$ & 0.612 & 2 \\
\hline Zootaxa (Online) & v. 1063 , fisrt page 1 , last page 31 & $1175-5334$ & 0.612 & 2 \\
\hline Zootaxa (Online) & v. 1065 , fistt page 1 , last page 27 & $1175-5334$ & 0.612 & 2 \\
\hline Zootaxa (Online) & v. 1079 , fistt page 1 , last page 8 & $1175-5334$ & 0.612 & 2 \\
\hline
\end{tabular}




\begin{tabular}{|c|c|c|c|c|}
\hline Journal & Paper info. & ISSN & IF & Half-Life \\
\hline Zootaxa (Online) & v. 1080 , fisrt page 39 , last page 64 & $1175-5334$ & 0.612 & 2 \\
\hline Zootaxa (Online) & v. 1092 , fisrt page 21 , last page 30 & $1175-5334$ & 0.612 & 2 \\
\hline Zootaxa (Online) & v. 1115 , fisrt page 61 , last page 68 & $1175-5334$ & 0.612 & 2 \\
\hline Zootaxa (Online) & v. 1123 , fisrt page 21 , last page 37 & 1175-5334 & 0.612 & 2 \\
\hline Zootaxa (Online) & v. 1125 , fistt page 57 , last page 68 & $1175-5334$ & 0.612 & 2 \\
\hline Zootaxa (Online) & v. 1131 , fistt page 33 , last page 48 & $1175-5334$ & 0.612 & 2 \\
\hline Zootaxa (Online) & v. 1135 , fistt page 57 , last page 68 & $1175-5334$ & 0.612 & 2 \\
\hline Zootaxa (Online) & v. 1136 , fistt page 49 , last page 64 & $1175-5334$ & 0.612 & 2 \\
\hline Zootaxa (Online) & v. 1167 , fistt page 17 , last page 30 & $1175-5334$ & 0.612 & 2 \\
\hline Zootaxa (Online) & v. 1167 , fistt page 47 , last page 60 & $1175-5334$ & 0.612 & 2 \\
\hline Zootaxa (Online) & v. 1174 , fistt page 49 , last page 62 & $1175-5334$ & 0.612 & 2 \\
\hline Zootaxa (Online) & v. 1174 , fist page 63 , last page 68 & $1175-5334$ & 0.612 & 2 \\
\hline Zootaxa (Online) & v. 1177 , fisrt page 57 , last page 68 & $1175-5334$ & 0.612 & 2 \\
\hline Zootaxa (Online) & v. 1197 , fisrt page 55 , last page 63 & $1175-5334$ & 0.612 & 2 \\
\hline Zootaxa (Online) & v. 1221 , fisrt page 25 , last page 28 & $1175-5334$ & 0.612 & 2 \\
\hline Zootaxa (Online) & v. 1235 , fistt page 63 , last page 68 & $1175-5334$ & 0.612 & 2 \\
\hline Zootaxa (Online) & v. 1249 , fistt page 61 , last page 68 & $1175-5334$ & 0.612 & 2 \\
\hline Zootaxa (Online) & v. 1259 , fistt page 55 , last page 59 & $1175-5334$ & 0.612 & 2 \\
\hline Zootaxa (Online) & v. 1299 , fisrt page 35 , last page 43 & $1175-5334$ & 0.612 & 2 \\
\hline Zootaxa (Online) & v. 1299 , fisrt page 45 , last page 55 & $1175-5334$ & 0.612 & 2 \\
\hline Zootaxa (Online) & v. 1326 , fisrt page 45 , last page 53 & $1175-5334$ & 0.612 & 2 \\
\hline Zootaxa (Online) & v. 1353, fisrt page 1 , last page 37 & $1175-5334$ & 0.612 & 2 \\
\hline Zootaxa (Online) & v. 1357, fisrt page 1 , last page 37 & $1175-5334$ & 0.612 & 2 \\
\hline Zootaxa (Online) & v. 1357 , fisrt page 61 , last page 68 & $1175-5334$ & 0.612 & 2 \\
\hline Zootaxa (Online) & v. 1363 , fisrt page 39 , last page 48 & $1175-5334$ & 0.612 & 2 \\
\hline Zootaxa (Online) & v. 1371 , fisrt page 23 , last page 35 & $1175-5334$ & 0.612 & 2 \\
\hline Zootaxa (Online) & v. 863, fisrt page 1, last page 7 & $1175-5334$ & 0.612 & 2 \\
\hline Zootaxa (Online) & v. 905 , fisrt page 1 , last page 11 & $1175-5334$ & 0.612 & 2 \\
\hline Zootaxa (Online) & v. 953, fisrt page 1 , last page 14 & $1175-5334$ & 0.612 & 2 \\
\hline Journal of Crustacean Biology & v. 24 , fisrt page 274 , last page 281 & $0278-0372$ & 0.608 & 9.8 \\
\hline Journal of Crustacean Biology & v. 24 , fisrt page 309 , last page 313 & $0278-0372$ & 0.608 & 9.8 \\
\hline Journal of Crustacean Biology & v. 25 , fisrt page 495 , last page 499 & 0278-0372 & 0.608 & 9.8 \\
\hline Journal of Crustacean Biology & v. 26 , fisrt page 248 , last page 257 & 0278-0372 & 0.608 & 9.8 \\
\hline Journal of Crustacean Biology & v. 26 , fisrt page 355 , last page 365 & 0278-0372 & 0.608 & 9.8 \\
\hline Journal of Crustacean Biology & v. 26, fisrt page 48, last page 54 & 0278-0372 & 0.608 & 9.8 \\
\hline $\begin{array}{l}\text { In Vitro Cellular \& Developmental Biology. } \\
\text { Plant }\end{array}$ & v. 40 , fisrt page 408 , last page 411 & $1054-5476$ & 0.607 & 5.5 \\
\hline In Vitro Cellular \& Developmental Biology. & v. 41 , fisrt page 806 , last page 811 & $1054-5476$ & & \\
\hline $\begin{array}{l}\text { Plant } \\
\text { In Vitro Cellular \& Developmental Biology. }\end{array}$ & 141 ficrt nage 832 lact nage 837 & $1054-5476$ & 0.607 & 5.5 \\
\hline Plant & V. 41, fisrt page 832 , last page $83 /$ & $1054-54 / 6$ & 0.607 & 5.5 \\
\hline $\begin{array}{l}\text { In Vitro Cellular \& Developmental Biology. } \\
\text { Plant }\end{array}$ & v. 42 , fisrt page 287 , last page 290 & $1054-5476$ & 0.607 & 5.5 \\
\hline In Vitro Cellular \& Developmental Biology. & v. 42 , fisrt page 50 , last page 53 & 1054-5476 & 0.001 & J. \\
\hline Plant & & & 0.607 & 5.5 \\
\hline $\begin{array}{l}\text { In Vitro Cellular \& Developmental Biology. } \\
\text { Plant }\end{array}$ & v. 42 , fisrt page 558 , last page 561 & $1054-5476$ & 0.607 & 5.5 \\
\hline Die Pharmazie (Berlin) & v. 59, fisrt page 965 , last page 967 & $0031-7144$ & 0.606 & 8.5 \\
\hline Die Pharmazie (Berlin) & v. 60 , fisrt page 13 , last page 17 & $0031-7144$ & 0.606 & 8.5 \\
\hline $\begin{array}{l}\text { Journal of Environmental Science and } \\
\text { Health. Part B, Pesticides, Food }\end{array}$ & v. 40 , fisrt page 151 , last page 157 & $0360-1234$ & & \\
\hline $\begin{array}{l}\text { Contaminants, and Agricultural Wastes } \\
\text { Journal of Environmental Science and } \\
\text { Health. Part B, Pesticides, Food }\end{array}$ & v. 41 , fistt page 1 , last page 11 & $0360-1234$ & 0.604 & 7.8 \\
\hline Contaminants, and Agricultural Wastes & & & 0.604 & 7.8 \\
\hline Marine and Freshwater Behaviour and & v. 37 , fisrt page 1 , last page 5 & $1023-6244$ & & \\
\hline Physiology ... & & & 0.604 & 6.9 \\
\hline Nova Hedwigia & v. 128 , fistt page 85 , last page 100 & 1438-9134 & 0.603 & 10 \\
\hline Nova Hedwigia (Berlin) & v. 79 , fisrt page 149 , last page 173 & $0029-5035$ & 0.603 & 10 \\
\hline Nova Hedwigia (Berlin) & v. 82 , fisrt page 105 , last page 113 & $0029-5035$ & 0.603 & 10 \\
\hline Nova Hedwigia (Berlin) & v. 82 , fisrt page 365 , last page 380 & 0029-5035 & 0.603 & 10 \\
\hline Anatomia Histologia Embryologia & v. 33 , fisrt page 183 , last page 187 & 0340-2096 & 0.593 & 5.5 \\
\hline $\begin{array}{l}\text { Bulletin of The European Association of } \\
\text { Fish Pathologists }\end{array}$ & v. 26 , fisrt page 271 , last page 274 & 0108-0288 & 0.581 & 7.6 \\
\hline
\end{tabular}




\begin{tabular}{|c|c|c|c|c|}
\hline Journal & Paper info. & ISSN & IF & Half-Life \\
\hline Annales de Limnologie & v. 42 , fisrt page 87, last page 96 & $0003-4088$ & 0.579 & 8.3 \\
\hline Genetics and Molecular Biology & v. 01, fisrt page 350 , last page 354 & $1415-4757$ & 0.574 & 5 \\
\hline Genetics and Molecular Biology & v. 01, fisrt page 45 , last page 50 & $1415-4757$ & 0.574 & 5 \\
\hline Genetics and Molecular Biology & v. 01, fisrt page 549 , last page 554 & $1415-4757$ & 0.574 & 5 \\
\hline Genetics and Molecular Biology & v. 05(2), fisrt page 256 , last page 261 & $1415-4757$ & 0.574 & 5 \\
\hline Genetics and Molecular Biology & v. 26 , fisrt page 741 , last page 746 & $1415-4757$ & 0.574 & 5 \\
\hline Genetics and Molecular Biology & v. 26 , fisrt page 750 , last page 754 & $1415-4757$ & 0.574 & 5 \\
\hline Genetics and Molecular Biology & v. 27, fisrt page 10 , last page 15 & $1415-4757$ & 0.574 & 5 \\
\hline Genetics and Molecular Biology & v. 27 , fisrt page 118 , last page 123 & $1415-4757$ & 0.574 & 5 \\
\hline Genetics and Molecular Biology & v. 27 , fisrt page 174 , last page 180 & $1415-4757$ & 0.574 & 5 \\
\hline Genetics and Molecular Biology & v. 27 , fisrt page 215 , last page 222 & $1415-4757$ & 0.574 & 5 \\
\hline Genetics and Molecular Biology & v. 27 , fisrt page 23 , last page 30 & $1415-4757$ & 0.574 & 5 \\
\hline Genetics and Molecular Biology & v. 27 , fistt page 253 , last page 258 & $1415-4757$ & 0.574 & 5 \\
\hline Genetics and Molecular Biology & v. 27, fistt page 33 , last page 37 & $1415-4757$ & 0.574 & 5 \\
\hline Genetics and Molecular Biology & v. 27 , fisrt page 378 , last page 384 & $1415-4757$ & 0.574 & 5 \\
\hline Genetics and Molecular Biology & v. 27 , fisrt page 505 , last page 510 & $1415-4757$ & 0.574 & 5 \\
\hline Genetics and Molecular Biology & v. 27 , fisrt page 51 , last page 54 & $1415-4757$ & 0.574 & 5 \\
\hline Genetics and Molecular Biology & v. 27 , fisrt page 561 , last page 566 & $1415-4757$ & 0.574 & 5 \\
\hline Genetics and Molecular Biology & v. 27, fisrt page 61 , last page 69 & $1415-4757$ & 0.574 & 5 \\
\hline Genetics and Molecular Biology & v. 27, fisrt page 70 , last page 73 & $1415-4757$ & 0.574 & 5 \\
\hline Genetics and Molecular Biology & v. 28 , fistt page 120 , last page 122 & $1415-4757$ & 0.574 & 5 \\
\hline Genetics and Molecular Biology & v. 28 , fistt page 175 , last page 179 & $1415-4757$ & 0.574 & 5 \\
\hline Genetics and Molecular Biology & v. 28 , fistt page 228 , last page 233 & $1415-4757$ & 0.574 & 5 \\
\hline Genetics and Molecular Biology & v. 28 , fistt page 248 , last page 253 & $1415-4757$ & 0.574 & 5 \\
\hline Genetics and Molecular Biology & v. 28 , fistt page 299 , last page 307 & $1415-4757$ & 0.574 & 5 \\
\hline Genetics and Molecular Biology & v. 28 , fistt page 308 , last page 313 & $1415-4757$ & 0.574 & 5 \\
\hline Genetics and Molecular Biology & v. 28 , fistt page 376 , last page 381 & $1415-4757$ & 0.574 & 5 \\
\hline Genetics and Molecular Biology & v. 28 , fistt page 444 , last page 446 & $1415-4757$ & 0.574 & 5 \\
\hline Genetics and Molecular Biology & v. 28 , fisrt page 46 , last page 53 & $1415-4757$ & 0.574 & 5 \\
\hline Genetics and Molecular Biology & v. 28 , fistt page 501 , last page 510 & $1415-4757$ & 0.574 & 5 \\
\hline Genetics and Molecular Biology & v. 28 , fistt page 511 , last page 519 & $1415-4757$ & 0.574 & 5 \\
\hline Genetics and Molecular Biology & v. 28 , fisrt page 548 , last page 554 & $1415-4757$ & 0.574 & 5 \\
\hline Genetics and Molecular Biology & v. 28 , fisrt page 555 , last page 561 & $1415-4757$ & 0.574 & 5 \\
\hline Genetics and Molecular Biology & v. 28, fisrt page 562 , last page 574 & $1415-4757$ & 0.574 & 5 \\
\hline Genetics and Molecular Biology & v. 28 , fisrt page 575 , last page 581 & $1415-4757$ & 0.574 & 5 \\
\hline Genetics and Molecular Biology & v. 28 , fisrt page 645 , last page 653 & $1415-4757$ & 0.574 & 5 \\
\hline Genetics and Molecular Biology & v. 28 , fisrt page 693 , last page 699 & $1415-4757$ & 0.574 & 5 \\
\hline Genetics and Molecular Biology & v. 28 , fisrt page 700 , last page 709 & $1415-4757$ & 0.574 & 5 \\
\hline Genetics and Molecular Biology & v. 28 , fisrt page 734 , last page 739 & $1415-4757$ & 0.574 & 5 \\
\hline Genetics and Molecular Biology & v. 28 , fisrt page 773 , last page 780 & $1415-4757$ & 0.574 & 5 \\
\hline Genetics and Molecular Biology & v. 28 , fisrt page 814 , last page 820 & $1415-4757$ & 0.574 & 5 \\
\hline Genetics and Molecular Biology & v. 28, fisrt page 833 , last page 838 & $1415-4757$ & 0.574 & 5 \\
\hline Genetics and Molecular Biology & v. 29 , fisrt page 207 , last page 214 & $1415-4757$ & 0.574 & 5 \\
\hline Genetics and Molecular Biology & v. 29 , fisrt page 215 , last page 219 & $1415-4757$ & 0.574 & 5 \\
\hline Genetics and Molecular Biology & v. 29 , fistt page 251 , last page 256 & $1415-4757$ & 0.574 & 5 \\
\hline Genetics and Molecular Biology & v. 29 , fistt page 257 , last page 262 & $1415-4757$ & 0.574 & 5 \\
\hline Genetics and Molecular Biology & v. 29 , fistt page 283 , last page 286 & $1415-4757$ & 0.574 & 5 \\
\hline Genetics and Molecular Biology & v. 29 , fistt page 566 , last page 571 & $1415-4757$ & 0.574 & 5 \\
\hline Genetics and Molecular Biology & v. 29, fistt page 62 , last page 66 & $1415-4757$ & 0.574 & 5 \\
\hline Genetics and Molecular Biology & v. 29 , fistt page 633 , last page 655 & $1415-4757$ & 0.574 & 5 \\
\hline Genetics and Molecular Biology & v. 29 , fistt page 659 , last page 664 & $1415-4757$ & 0.574 & 5 \\
\hline Genetics and Molecular Biology & v. 29 , fistt page 735 , last page 740 & $1415-4757$ & 0.574 & 5 \\
\hline Genetics and Molecular Biology & v. 29 , fistt page 750 , last page 754 & $1415-4757$ & 0.574 & 5 \\
\hline Genetics and Molecular Biology & v. 29, fisrt page 97 , last page 104 & $1415-4757$ & 0.574 & 5 \\
\hline Genetics and Molecular Biology & v. 4 , fisrt page 505 , last page 510 & $1415-4757$ & 0.574 & 5 \\
\hline Genetics and Molecular Biology & v. 47 , fisrt page 495 , last page 501 & $1415-4757$ & 0.574 & 5 \\
\hline Genetics and Molecular Biology & v. 5 , fisrt page 154 , last page 168 & $1415-4757$ & 0.574 & 5 \\
\hline Ameghiniana & v. 41 , fisrt page 521 , last page 534 & $0002-7014$ & 0.573 & 10 \\
\hline Ameghiniana & v. 42 , fistt page 705 , last page 723 & $0002-7014$ & 0.573 & 10 \\
\hline Ameghiniana & v. 43 , fisrt page 591 , last page 609 & $0002-7014$ & 0.573 & 10 \\
\hline
\end{tabular}




\begin{tabular}{l} 
Journal \\
\hline Acta Theriologica \\
Acta Theriologica \\
Acta Theriologica \\
Acta Theriologica \\
Genetic Resources and Crop Evolution
\end{tabular}

Genetic Resources and Crop Evolution Genetic Resources and Crop Evolution Genetic Resources and Crop Evolution Genetic Resources and Crop Evolution Genetic Resources and Crop Evolution Fish Physiology and Biochemistry

\section{Entomological News}

Entomological News

Journal of the New York Entomological Society

Raffles Bulletin of Zoology

Acta Physiologiae Plantarum

Biocell (Mendoza)

Biocell (Mendoza)

Biocell (Mendoza)

Tropical Zoology

Neotropical Ichthyology

Neotropical Ichthyology

Neotropical Ichthyology

Neotropical Ichthyology

Neotropical Ichthyology

Neotropical Ichthyology

Neotropical Ichthyology

Neotropical Ichthyology

Neotropical Ichthyology

Neotropical Ichthyology

Neotropical Ichthyology

Neotropical Ichthyology

Neotropical Ichthyology

Neotropical Ichthyology

Neotropical Ichthyology

Neotropical Ichthyology

Neotropical Ichthyology

Neotropical Ichthyology

Neotropical Ichthyology

Neotropical Ichthyology

Neotropical Ichthyology

Neotropical Ichthyology

Neotropical Ichthyology

Neotropical Ichthyology

Neotropical Ichthyology

Neotropical Ichthyology

Neotropical Ichthyology

Neotropical Ichthyology

Neotropical Ichthyology

Neotropical Ichthyology

Neotropical Ichthyology

Neotropical Ichthyology

Neotropical Ichthyology

Neotropical Ichthyology

Neotropical Ichthyology

Neotropical Ichthyology

Neotropical Ichthyology

Neotropical Ichthyology

Neotropical Ichthyology
Paper info.

v. 50, fisrt page 551, last page 560

v. 51 , fisrt page 163 , last page 168

v. 51 , fisrt page 53 , last page 60

v. 51 , fisrt page 69 , last page 74

v. 51 , fist page 01 , last page 08

v. 51 , fisrt page 299, last page 307

v. 51 , fisrt page 871 , last page 882

v. 52, fist page 1079, last page 1086

v. 53, fisrt page 541, last page 552

v. 53, fisrt page 975 , last page 983

v. 32 , fisrt page 105, last page 112

v. 104 , fisrt page 218 , last page 224

v. 116, fist page 39, last page 58

v. 111 , fisrt page 242 , last page 252

v. 52, fisrt page 1, last page 6

v. 27 , fisrt page 387 , last page 393

v. 28, fisrt page 165, last page 169

v. 28 , fisrt page 21 , last page 30

v. 30 , fisrt page 111 , last page 118

v. 18 , fisrt page 234 , last page 237

v. 01 , fisrt page 123 , last page 125

v. 1 , fisrt page 277 , last page 284

v. 1 , fisrt page 499, last page 508

v. 2, fisrt page 103, last page 105

v. 2 , fist page 107 , last page 110

v. 2, fisrt page 109, last page 115

v. 2, fisrt page 117 , last page 122

v. 2, fisrt page 123, last page 125

v. 2 , fisrt page 13 , last page 17

v. 2, fisrt page 137, last page 144

v. 2, fisrt page 151 , last page 156

v. 2, fisrt page 163, last page 166

v. 2 , fist page 19 , last page 30

v. 2, fisrt page 205, last page 208

v. 2 , fisrt page 209 , last page 215

v. 2 , fisrt page 31 , last page 36

v. 2 , fisrt page 61 , last page 74

v. 2 , fist page 9 , last page 12

v. 2 , fisrt page 93 , last page 98

v. 2, fist page 99, last page 102

v. 3, fisrt page 1 , last page 60

v. 3, fisrt page 111, last page 114

v. 3, fisrt page 123, last page 125

v. 3, fisrt page 127 , last page 134

v. 3, fisrt page 271 , last page 276

v. 3, fisrt page 291, last page 298

v. 3, fisrt page 299, last page 304

v. 3, fisrt page 305 , last page 311

v. 3, fisrt page 315 , last page 318

v. 3 , fisrt page 401 , last page 410

v. 3 , fisrt page 411 , last page 419

v. 3 , fisrt page 427 , last page 432

v. 3 , fisrt page 433 , last page 438

v. 3 , fist page 445 , last page 448

v. 3, fisrt page 449 , last page 452

v. 3 , fisrt page 465 , last page 472

v. 3 , fisrt page 509 , last page 518

v. 3 , fisrt page 579 , last page 585

v. 3 , fisrt page 587 , last page 595
ISSN

0001-705

$0001-7051$

0001-7051

0001-7051

0925-9864

0925-9864

0925-9864

0925-9864

0925-9864

0925-9864

0920-1742

0013-872X

0013-872X

0028-7199

0217-2445

0137-5881

0327-9545

0327-9545

0327-9545

0394-6975

1679-6225

$1679-6225$

$1679-6225$

1679-6225

1679-6225

1679-6225

1679-6225

1679-6225

1679-6225

1679-6225

1679-6225

1679-6225

1679-6225

1679-6225

1679-6225

1679-6225

1679-6225

$1679-6225$

$1679-6225$

1679-6225

1679-6225

1679-6225

1679-6225

1679-6225

1679-6225

1679-6225

1679-6225

1679-6225

1679-6225

1679-6225

1679-6225

1679-6225

1679-6225

$1679-6225$

$1679-6225$

1679-6225

$1679-6225$

1679-6225

1679-6225

$\begin{array}{cr}\text { IF } & \text { Half-Life } \\ 0.571 & 10\end{array}$

$0.571 \quad 10$

$0.571 \quad 10$

$0.571 \quad 10$

$0.569 \quad 5.6$

$0.569 \quad 5.6$

$0.569 \quad 5.6$

$0.569 \quad 5.6$

$0.569 \quad 5.6$

$0.569 \quad 5.6$

$0.558 \quad 8.4$

$0.556 \quad 10$

$0.556 \quad 10$

$0.556 \quad 10$

$0.533 \quad 5.4$

$0.528 \quad 6.9$

$0.526 \quad 4.8$

$0.526 \quad 4.8$

$0.526 \quad 4.8$

$0.522 \quad 10$

0.512

0.512

0.512

0.512

0.512

0.512

0.512

0.512

0.512

0.512

0.512

0.512

0.512

0.512

0.512

0.512

0.512

0.512

0.512

0.512

0.512

0.512

0.512

0.512

0.512

0.512

0.512

0.512

0.512

0.512

0.512

0.512

0.512

0.512

0.512

0.512

0.512

0.512

0.512 
Journa

Neotropical Ichthyology

Neotropical Ichthyology

Neotropical Ichthyology

Neotropical Ichthyology

Neotropical Ichthyology

Neotropical Ichthyology

Neotropical Ichthyology

Neotropical Ichthyology

Neotropical Ichthyology

Neotropical Ichthyology

Neotropical Ichthyology

Neotropical Ichthyology

Neotropical Ichthyology

Neotropical Ichthyology

Neotropical Ichthyology

Neotropical Ichthyology

Neotropical Ichthyology

Neotropical Ichthyology

Neotropical Ichthyology

Neotropical Ichthyology

Neotropical Ichthyology

Neotropical Ichthyology

Neotropical Ichthyology

Neotropical Ichthyology

Neotropical Ichthyology

Neotropical Ichthyology

Neotropical Ichthyology

Neotropical Ichthyology

Neotropical Ichthyology

Neotropical Ichthyology

Neotropical Ichthyology

Neotropical Ichthyology

Neotropical Ichthyology

Neotropical Ichthyology

Neotropical Ichthyology

Neotropical Ichthyology

Neotropical Ichthyology

Journal of Radioanalytical and Nuclear

Chemistry

European Journal of Wildlife Research

Bulletin of Environmental Contamination and Toxicology

Bulletin of Environmental Contamination and Toxicology

Bulletin of Environmental Contamination and Toxicology

Bulletin of Environmental Contamination and Toxicology

Bulletin of Environmental Contamination and Toxicology

Helminthologia (Bratislava)

Waterbirds

Waterbirds

Waterbirds

Entomological Science (Tokyo)

Entomological Science (Tokyo)

Cahiers de Biologie Marine

Cahiers de Biologie Marine

Cahiers de Biologie Marine

Cryptogamie. Algologie

Cryptogamie. Algologie
Paper info.

v. 3, fisrt page 61, last page 67

v. 3 , fist page 69 , last page 82

v. 3 , fist page 83 , last page 88

v. 4 , fisrt page 1 , last page 26

v. 4 , fisrt page 107 , last page 118

v. 4, fisrt page 119, last page 122

v. 4 , fisrt page 123 , last page 126

v. 4 , fisrt page 127 , last page 146

v. 4 , fisrt page 147 , last page 156

v. 4, fisrt page 157, last page 166

v. 4, fisrt page 167, last page 196

v. 4, fisrt page 197, last page 202

v. 4 , fisrt page 203 , last page 214

v. 4 , fisrt page 219 , last page 224

v. 4 , fisrt page 269 , last page 278

v. 4 , fisrt page 279 , last page 284

v. 4 , fisrt page 287 , last page 290

v. 4, fisrt page 301 , last page 308

v. 4 , fisrt page 309 , last page 318

v. 4 , fisrt page 329 , last page 337

v. 4 , fisrt page 339 , last page 348

v. 4, fisrt page 377 , last page 379

v. 4 , fisrt page 379 , last page 383

v. 4 , fisrt page 411 , last page 418

v. 4 , fisrt page 419 , last page 426

v. 4 , fisrt page 427 , last page 433

v. 4 , fisrt page 435 , last page 440

v. 4 , fisrt page 441 , last page 445

v. 4 , fisrt page 45 , last page 52

v. 4 , fisrt page 451 , last page 455

v. 4 , fisrt page 455 , last page 464

v. 4 , fisrt page 457 , last page 460

v. 4 , fisrt page 461 , last page 463

v. 4 , fist page 463 , last page 465

v. 4 , fisrt page 53 , last page 60

v. 4 , fisrt page 56 , last page 61

v. 4 , fisrt page 69 , last page 72

v. 4 , fisrt page 99 , last page 105

v. 259 , fisrt page 59 , last page 63

v. 51, fisrt page 199, last page 10206

v. 72 , fisrt page 178 , last page 185

v. 72 , fisrt page 639 , last page 646

v. 74 , fisrt page 582 , last page 588

v. 74 , fisrt page 793 , last page 800

v. 76 , fisrt page 505 , last page 511

v. 43 , fisrt page 158 , last page 160

v. 28 , fisrt page 110 , last page 119

v. 28, fisrt page 261, last page 272

v. 29, fisrt page 335 , last page 344

v. 08 , fisrt page 05 , last page 07

v. 8 , fist page 33 , last page 39

v. 45 , fisrt page 243 , last page 254

v. 46 , fisrt page 69 , last page 80

v. 47 , fisrt page 261 , last page 270

v. 26 , fisrt page 381 , last page 397

v. 27 , fisrt page 1 , last page 14
ISSN

1679-6225

$1679-6225$

$1679-6225$

$1679-6225$

1679-6225

1679-6225

1679-6225

$1679-6225$

1679-6225

1679-6225

1679-6225

1679-6225

1679-6225

1679-6225

1679-6225

1679-6225

1679-6225

1679-6225

1679-6225

1679-6225

1679-6225

1679-6225

1679-6225

1679-6225

1679-6225

1679-6225

1679-6225

1679-6225

1679-6225

1679-6225

1679-6225

1679-6225

1679-6225

1679-6225

1679-6225

1679-6225

1679-6225

1679-6225

0236-5731

1612-4642

0007-4861

0007-4861

0007-4861

0007-4861

0007-4861

0440-6605

1524-4695

1524-4695

1524-4695

1343-8786

1343-8786

0007-9723

0007-9723

0007-9723

0181-1568

0181-1568
IF

0.512

0.512

0.512

0.512

0.512

0.512

0.512

0.512

0.512

0.512

0.512

0.512

0.512

0.512

0.512

0.512

0.512

0.512

0.512

0.512

0.512

0.512

0.512

0.512

0.512

0.512

0.512

0.512

0.512

0.512

0.512

0.512

0.512

0.512

0.512

0.512

0.512

0.509

0.506

0.505

0.505

0.505

0.505

0.505

0.5

0.497

0.497

0.497

0.495

0.495

0.492

0.492

0.492

0.491

0.491
9.9

9.9

9.9

9.9

9.9

\section{6}

7.5

4.8

4.8

4.8

5.7

5.7

10

10

10

6.6

6.6 


\begin{tabular}{|c|c|c|c|c|}
\hline Journal & Paper info. & ISSN & IF & Half-Life \\
\hline Cryptogamie. Algologie & v. 27 , fisrt page 265 , last page 277 & $0181-1568$ & 0.491 & 6.6 \\
\hline Cybium (Paris) & v. 28 , fisrt page 47 , last page 53 & 0399-0974 & 0.491 & 10 \\
\hline Cybium (Paris) & v. 30 , fisrt page 1 , last page 3 & 0399-0974 & 0.491 & 10 \\
\hline Mycotaxon & v. 54 , fisrt page 15 , last page 19 & $0093-4666$ & 0.486 & 10 \\
\hline Mycotaxon & v. 8 , fistt page 349 , last page 353 & $0093-4666$ & 0.486 & 10 \\
\hline Mycotaxon & v. 88 , fisrt page 107 , last page 112 & $0093-4666$ & 0.486 & 10 \\
\hline Mycotaxon & v. 89 , fisrt page 355 , last page 378 & $0093-4666$ & 0.486 & 10 \\
\hline Mycotaxon & v. 92 , fisrt page 103 , last page 106 & $0093-4666$ & 0.486 & 10 \\
\hline Mycotaxon & v. 94 , fisrt page 293 , last page 301 & $0093-4666$ & 0.486 & 10 \\
\hline Mycotaxon & v. 95 , fisrt page 117 , last page 131 & $0093-4666$ & 0.486 & 10 \\
\hline Mycotaxon & v. 95 , fisrt page 241 , last page 245 & $0093-4666$ & 0.486 & 10 \\
\hline Mycotaxon & v. 95 , fisrt page 261 , last page 270 & $0093-4666$ & 0.486 & 10 \\
\hline Mycotaxon & v. 95 , fisrt page 295 , last page 300 & $0093-4666$ & 0.486 & 10 \\
\hline Mycotaxon & v. 96, fisrt page 129, last page 132 & $0093-4666$ & 0.486 & 10 \\
\hline Mycotaxon & v. 96 , fisrt page 241 , last page 252 & $0093-4666$ & 0.486 & 10 \\
\hline Mycotaxon & v. 96, fisrt page 327 , last page 332 & $0093-4666$ & 0.486 & 10 \\
\hline Mycotaxon & v. 97, fisrt page 1, last page 4 & $0093-4666$ & 0.486 & 10 \\
\hline Mycotaxon & v. 97, fisrt page 219 , last page 229 & $0093-4666$ & 0.486 & 10 \\
\hline Allelopathy Journal & v. 13 , fisrt page 201 , last page 210 & $0971-4693$ & 0.48 & 4 \\
\hline Revue Suisse de Zoologie & v. 112 , fistt page 559 , last page 572 & $0035-418 X$ & 0.474 & 10 \\
\hline Journal of the Kansas Entomological & v. 77 , fisrt page 470 , last page 483 & $0022-8567$ & & \\
\hline Society & & & 0.472 & 10 \\
\hline $\begin{array}{l}\text { Journal of the Kansas Entomological } \\
\text { Society }\end{array}$ & v. 78 , fisrt page 381 , last page 386 & $0022-8567$ & 0.472 & 10 \\
\hline World Journal of Microbiology and & v. 20 , fisrt page 371 , last page 375 & 0959-3993 & & 10 \\
\hline Biotechnology & & & 0.471 & 6.7 \\
\hline World Journal of Microbiology and & v. 21 , fisrt page 151 , last page 154 & 0959-3993 & & \\
\hline Biotechnology & & & 0.471 & 6.7 \\
\hline World Journal of Microbiology and & v. 21 , fisrt page 189 , last page 192 & 0959-3993 & & \\
\hline Biotechnology & & & 0.471 & 6.7 \\
\hline World Journal of Microbiology and & v. 21 , fisrt page 297 , last page 301 & $0959-3993$ & & \\
\hline $\begin{array}{l}\text { Biotechnology } \\
\text { World Journal of Microbiology and }\end{array}$ & v. 21 , fisrt page 655 , last page 659 & 0959-3993 & 0.471 & 6.7 \\
\hline Biotechnology & V. LT, mart page ous, last page ous & (2) & 0.471 & 6.7 \\
\hline World Journal of Microbiology and & v. 22, fisrt page 959, last page 965 & 0959-3993 & & 67 \\
\hline Sociobiology & v. 25 , fisrt page 413 , last page 432 & 0361-6525 & $\begin{array}{l}0.4 / 1 \\
0.459\end{array}$ & 7.3 \\
\hline Sociobiology & v. 41 , fisrt page 141 , last page 149 & $0361-6525$ & 0.459 & 7.3 \\
\hline Sociobiology & v. 43 , fisrt page 377 , last page 387 & $0361-6525$ & 0.459 & 7.3 \\
\hline Sociobiology & v. 43 , fisrt page 467 , last page 475 & $0361-6525$ & 0.459 & 7.3 \\
\hline Sociobiology & v. 43 , fisrt page 551 , last page 555 & $0361-6525$ & 0.459 & 7.3 \\
\hline Sociobiology & v. 44, fisrt page 109, last page 122 & $0361-6525$ & 0.459 & 7.3 \\
\hline Sociobiology & v. 44 , fisrt page 123 , last page 126 & $0361-6525$ & 0.459 & 7.3 \\
\hline Sociobiology & v. 44 , fisrt page 271 , last page 282 & $0361-6525$ & 0.459 & 7.3 \\
\hline Sociobiology & v. 44 , fisrt page 379 , last page 390 & $0361-6525$ & 0.459 & 7.3 \\
\hline Sociobiology & v. 44, fisrt page 391 , last page 402 & $0361-6525$ & 0.459 & 7.3 \\
\hline Sociobiology & v. 44 , fisrt page 511 , last page 518 & $0361-6525$ & 0.459 & 7.3 \\
\hline Sociobiology & v. 44 , fisrt page 599 , last page 606 & $0361-6525$ & 0.459 & 7.3 \\
\hline Sociobiology & v. 44 , fisrt page 607 , last page 613 & $0361-6525$ & 0.459 & 7.3 \\
\hline Sociobiology & v. 44, fisrt page 615 , last page 622 & $0361-6525$ & 0.459 & 7.3 \\
\hline Sociobiology & v. 44 , fisrt page 707 , last page 716 & $0361-6525$ & 0.459 & 7.3 \\
\hline Sociobiology & v. 45, fisrt page 01 , last page 11 & $0361-6525$ & 0.459 & 7.3 \\
\hline Sociobiology & v. 45 , fisrt page 105 , last page 118 & $0361-6525$ & 0.459 & 7.3 \\
\hline Sociobiology & v. 45 , fisrt page 137 , last page 139 & $0361-6525$ & 0.459 & 7.3 \\
\hline Sociobiology & v. 45 , fisrt page 195 , last page 204 & $0361-6525$ & 0.459 & 7.3 \\
\hline Sociobiology & v. 45 , fisrt page 271 , last page 305 & $0361-6525$ & 0.459 & 7.3 \\
\hline Sociobiology & v. 45, fisrt page 31 , last page 51 & $0361-6525$ & 0.459 & 7.3 \\
\hline Sociobiology & v. 45 , fisrt page 347 , last page 353 & $0361-6525$ & 0.459 & 7.3 \\
\hline Sociobiology & v. 45 , fisrt page 377 , last page 387 & $0361-6525$ & 0.459 & 7.3 \\
\hline Sociobiology & v. 45 , fisrt page 389 , last page 399 & $0361-6525$ & 0.459 & 7.3 \\
\hline Sociobiology & v. 45 , fisrt page 449 , last page 462 & $0361-6525$ & 0.459 & 7.3 \\
\hline Sociobiology & v. 45 , fisrt page 659 , last page 669 & $0361-6525$ & 0.459 & 7.3 \\
\hline Sociobiology & v. 45 , fisrt page 797 , last page 809 & $0361-6525$ & 0.459 & 7.3 \\
\hline
\end{tabular}




\begin{tabular}{|c|c|c|c|c|}
\hline Journal & Paper info. & ISSN & IF & Half-Life \\
\hline Sociobiology & v. 45, fisrt page 9, last page 14 & $0361-6525$ & 0.459 & 7.3 \\
\hline Sociobiology & v. 45 , fisrt page 99, last page 103 & $0361-6525$ & 0.459 & 7.3 \\
\hline Sociobiology & v. 46 , fisrt page 17 , last page 26 & $0361-6525$ & 0.459 & 7.3 \\
\hline Sociobiology & v. 46 , fisrt page 185 , last page 193 & $0361-6525$ & 0.459 & 7.3 \\
\hline Sociobiology & v. 46 , fisrt page 279 , last page 288 & $0361-6525$ & 0.459 & 7.3 \\
\hline Sociobiology & v. 46 , fisrt page 317 , last page 327 & $0361-6525$ & 0.459 & 7.3 \\
\hline Sociobiology & v. 46 , fisrt page 329 , last page 334 & $0361-6525$ & 0.459 & 7.3 \\
\hline Sociobiology & v. 46 , fisrt page 535 , last page 550 & $0361-6525$ & 0.459 & 7.3 \\
\hline Sociobiology & v. 46 , fisrt page 579 , last page 593 & $0361-6525$ & 0.459 & 7.3 \\
\hline Sociobiology & v. 46 , fisrt page 627 , last page 636 & $0361-6525$ & 0.459 & 7.3 \\
\hline Sociobiology & v. 46, fisrt page 87 , last page 103 & $0361-6525$ & 0.459 & 7.3 \\
\hline Sociobiology & v. 47 , fisrt page 115 , last page 123 & $0361-6525$ & 0.459 & 7.3 \\
\hline Sociobiology & v. 47 , fisrt page 125 , last page 134 & $0361-6525$ & 0.459 & 7.3 \\
\hline Sociobiology & v. 47 , fisrt page 135 , last page 147 & $0361-6525$ & 0.459 & 7.3 \\
\hline Sociobiology & v. 47 , fisrt page 165 , last page 174 & $0361-6525$ & 0.459 & 7.3 \\
\hline Sociobiology & v. 47 , fisrt page 215 , last page 223 & $0361-6525$ & 0.459 & 7.3 \\
\hline Sociobiology & v. 47 , fisrt page 225 , last page 234 & $0361-6525$ & 0.459 & 7.3 \\
\hline Sociobiology & v. 47 , fisrt page 235 , last page 252 & $0361-6525$ & 0.459 & 7.3 \\
\hline Sociobiology & v. 47 , fisrt page 265 , last page 274 & $0361-6525$ & 0.459 & 7.3 \\
\hline Sociobiology & v. 47 , fisrt page 275 , last page 287 & $0361-6525$ & 0.459 & 7.3 \\
\hline Sociobiology & v. 47, fisrt page 391 , last page 399 & $0361-6525$ & 0.459 & 7.3 \\
\hline Sociobiology & v. 47 , fisrt page 415 , last page 421 & $0361-6525$ & 0.459 & 7.3 \\
\hline Sociobiology & v. 47 , fisrt page 423 , last page 431 & $0361-6525$ & 0.459 & 7.3 \\
\hline Sociobiology & v. 47, fisrt page 483 , last page 494 & $0361-6525$ & 0.459 & 7.3 \\
\hline Sociobiology & v. 47 , fisrt page 543 , last page 552 & $0361-6525$ & 0.459 & 7.3 \\
\hline Sociobiology & v. 47 , fisrt page 547 , last page 570 & $0361-6525$ & 0.459 & 7.3 \\
\hline Sociobiology & v. 47 , fisrt page 739 , last page 749 & $0361-6525$ & 0.459 & 7.3 \\
\hline Sociobiology & v. 47 , fisrt page 759 , last page 770 & $0361-6525$ & 0.459 & 7.3 \\
\hline Sociobiology & v. 47 , fisrt page 829 , last page 842 & $0361-6525$ & 0.459 & 7.3 \\
\hline Sociobiology & v. 47 , fisrt page 843 , last page 858 & $0361-6525$ & 0.459 & 7.3 \\
\hline Sociobiology & v. 47 , fisrt page 859 , last page 872 & $0361-6525$ & 0.459 & 7.3 \\
\hline Sociobiology & v. 48 , fisrt page 135 , last page 153 & $0361-6525$ & 0.459 & 7.3 \\
\hline Sociobiology & v. 48, fisrt page 21, last page 26 & $0361-6525$ & 0.459 & 7.3 \\
\hline Sociobiology & v. 48 , fisrt page 365 , last page 374 & $0361-6525$ & 0.459 & 7.3 \\
\hline Sociobiology & v. 48 , fisrt page 503 , last page 513 & $0361-6525$ & 0.459 & 7.3 \\
\hline Sociobiology & v. 48 , fisrt page 515 , last page 526 & $0361-6525$ & 0.459 & 7.3 \\
\hline Sociobiology & v. 48 , fisrt page 553 , last page 558 & $0361-6525$ & 0.459 & 7.3 \\
\hline Sociobiology & v. 48 , fisrt page 673 , last page 680 & $0361-6525$ & 0.459 & 7.3 \\
\hline Sociobiology & v. 48 , fisrt page 717 , last page 726 & $0361-6525$ & 0.459 & 7.3 \\
\hline Sociobiology & v. 48 , fisrt page 759 , last page 770 & $0361-6525$ & 0.459 & 7.3 \\
\hline Sociobiology & v. n. fisrt page 934 , last page 947 & $0361-6525$ & 0.459 & 7.3 \\
\hline Economic Botany & v. 59, fisrt page 77 , last page 86 & 0013-0001 & 0.449 & 10 \\
\hline Journal of Arachnology & v. 10 , fisrt page 113 , last page 127 & 0161-8202 & 0.448 & 7.9 \\
\hline Journal of Arachnology & v. 32, fisrt page 188, last page 191 & 0161-8202 & 0.448 & 7.9 \\
\hline Journal of Arachnology & v. 32 , fisrt page 208 , last page 220 & 0161-8202 & 0.448 & 7.9 \\
\hline Journal of Arachnology & v. 32, fisrt page 22 , last page 30 & 0161-8202 & 0.448 & 7.9 \\
\hline Journal of Arachnology & v. 32 , fisrt page 332 , last page 336 & 0161-8202 & 0.448 & 7.9 \\
\hline Journal of Arachnology & v. 32 , fisrt page 345 , last page 348 & 0161-8202 & 0.448 & 7.9 \\
\hline Journal of Arachnology & v. 33, fisrt page 290, last page 299 & 0161-8202 & 0.448 & 7.9 \\
\hline Journal of Arachnology & v. 33 , fisrt page 456 , last page 467 & 0161-8202 & 0.448 & 7.9 \\
\hline Journal of Arachnology & v. 33 , fisrt page 591 , last page 596 & 0161-8202 & 0.448 & 7.9 \\
\hline Journal of Arachnology & v. 33 , fisrt page 670 , last page 680 & 0161-8202 & 0.448 & 7.9 \\
\hline Journal of Arachnology & v. 33 , fisrt page 758 , last page 766 & 0161-8202 & 0.448 & 7.9 \\
\hline Journal of Arachnology & v. 33 , fisrt page 873 , last page 877 & 0161-8202 & 0.448 & 7.9 \\
\hline Journal of Arachnology & v. 34, fisrt page 186, last page 193 & 0161-8202 & 0.448 & 7.9 \\
\hline Journal of Arachnology & v. 34 , fisrt page 435 , last page 443 & 0161-8202 & 0.448 & 7.9 \\
\hline Journal of Arachnology & v. 36 , fisrt page 214 , last page 220 & 0161-8202 & 0.448 & 7.9 \\
\hline Journal of Plant Nutrition & v. 28 , fisrt page 2101 , last page 2112 & $0190-4167$ & 0.441 & 9.6 \\
\hline Journal of Plant Nutrition & v. 28 , fisrt page 507 , last page 520 & $0190-4167$ & 0.441 & 9.6 \\
\hline Israel Journal of Plant Sciences & v. 52 , fisrt page 205, last page 212 & 0792-9978 & 0.437 & 6.5 \\
\hline
\end{tabular}




\begin{tabular}{|c|c|c|c|c|}
\hline Journal & Paper info. & ISSN & IF & Half-Life \\
\hline Aquatic Insects & v. 26 , fistt page 191 , last page 197 & $0165-0424$ & 0.431 & 10 \\
\hline Aquatic Insects & v. 26 , fisrt page 281 , last page 288 & 0165-0424 & 0.431 & 10 \\
\hline Aquatic Insects & v. 26 , fisrt page 53 , last page 63 & 0165-0424 & 0.431 & 10 \\
\hline Aquatic Insects & v. 26 , fisrt page 97 , last page 114 & 0165-0424 & 0.431 & 10 \\
\hline Crustaceana (Leiden) & v. 76 , fisrt page 187, last page 192 & $0011-216 X$ & 0.421 & 10 \\
\hline Crustaceana (Leiden) & v. 76 , fisrt page 625 , last page 630 & $0011-216 x$ & 0.421 & 10 \\
\hline Crustaceana (Leiden) & v. 77 , fisrt page 941 , last page 954 & $0011-216 X$ & 0.421 & 10 \\
\hline Crustaceana (Leiden) & v. 78 , fisrt page 975 , last page 986 & $0011-216 X$ & 0.421 & 10 \\
\hline Journal of Entomological Science & v. 40 , fisrt page 347 , last page 351 & 0749-8004 & 0.42 & 10 \\
\hline Journal of Entomological Science & v. 9 , fisrt page 13, last page 17 & 0749-8004 & 0.42 & 10 \\
\hline Pesquisa Veterinária Brasileira & v. 24 , fisrt page 15 , last page 17 & $0100-736 x$ & 0.42 & 6.5 \\
\hline Pesquisa Veterinária Brasileira & v. 24, fisrt page 191, last page 198 & $0100-736 X$ & 0.42 & 6.5 \\
\hline Invertebrate Reproduction \& Development & v. 45 , fisrt page 15 , last page 28 & 0792-4259 & 0.419 & 9 \\
\hline Invertebrate Reproduction \& Development & v. 45 , fisrt page 221 , last page 230 & 0792-4259 & 0.419 & 9 \\
\hline Invertebrate Reproduction \& Development & v. 45 , fisrt page 29 , last page 39 & $0792-4259$ & 0.419 & 9 \\
\hline Neotropical Entomology & v. $1(1)$, fist page 24 , last page 28 & $1519-566 x$ & 0.413 & 3.3 \\
\hline Neotropical Entomology & v. 1, fisrt page 644 , last page 653 & $1519-566 X$ & 0.413 & 3.3 \\
\hline Neotropical Entomology & v. 33 , fisrt page 179 , last page 187 & $1519-566 X$ & 0.413 & 3.3 \\
\hline Neotropical Entomology & v. 33 , fisrt page 231 , last page 236 & $1519-566 X$ & 0.413 & 3.3 \\
\hline Neotropical Entomology & v. 33 , fisrt page 237 , last page 241 & $1519-566 X$ & 0.413 & 3.3 \\
\hline Neotropical Entomology & v. 33 , fisrt page 267 , last page 269 & $1519-566 X$ & 0.413 & 3.3 \\
\hline Neotropical Entomology & v. 33 , fisrt page 29 , last page 33 & $1519-566 X$ & 0.413 & 3.3 \\
\hline Neotropical Entomology & v. 33 , fisrt page 327 , last page 332 & $1519-566 x$ & 0.413 & 3.3 \\
\hline Neotropical Entomology & v. 33, fisrt page 333 , last page 336 & $1519-566 x$ & 0.413 & 3.3 \\
\hline Neotropical Entomology & v. 33 , fisrt page 337 , last page 340 & $1519-566 x$ & 0.413 & 3.3 \\
\hline Neotropical Entomology & v. 33 , fisrt page 35 , last page 38 & $1519-566 x$ & 0.413 & 3.3 \\
\hline Neotropical Entomology & v. 33, fisrt page 39 , last page 42 & $1519-566 x$ & 0.413 & 3.3 \\
\hline Neotropical Entomology & v. 33 , fisrt page 427 , last page 431 & $1519-566 x$ & 0.413 & 3.3 \\
\hline Neotropical Entomology & v. 33, fisrt page 43, last page 49 & $1519-566 X$ & 0.413 & 3.3 \\
\hline Neotropical Entomology & v. 33, fisrt page 433 , last page 437 & $1519-566 X$ & 0.413 & 3.3 \\
\hline Neotropical Entomology & v. 33 , fisrt page 439 , last page 446 & $1519-566 X$ & 0.413 & 3.3 \\
\hline Neotropical Entomology & v. 33, fisrt page 451 , last page 455 & $1519-566 X$ & 0.413 & 3.3 \\
\hline Neotropical Entomology & v. 33 , fisrt page 457 , last page 462 & $1519-566 X$ & 0.413 & 3.3 \\
\hline Neotropical Entomology & v. 33 , fisrt page 469 , last page 479 & $1519-566 X$ & 0.413 & 3.3 \\
\hline Neotropical Entomology & v. 33 , fisrt page 487 , last page 492 & $1519-566 X$ & 0.413 & 3.3 \\
\hline Neotropical Entomology & v. 33 , fisrt page 511 , last page 513 & $1519-566 X$ & 0.413 & 3.3 \\
\hline Neotropical Entomology & v. 33 , fisrt page 557 , last page 562 & $1519-566 X$ & 0.413 & 3.3 \\
\hline Neotropical Entomology & v. 33 , fisrt page 563 , last page 567 & $1519-566 X$ & 0.413 & 3.3 \\
\hline Neotropical Entomology & v. 33 , fisrt page 583 , last page 588 & $1519-566 X$ & 0.413 & 3.3 \\
\hline Neotropical Entomology & v. 33, fisrt page 625 , last page 629 & $1519-566 X$ & 0.413 & 3.3 \\
\hline Neotropical Entomology & v. 33 , fisrt page 65 , last page 70 & $1519-566 X$ & 0.413 & 3.3 \\
\hline Neotropical Entomology & v. 33 , fisrt page 661 , last page 664 & $1519-566 X$ & 0.413 & 3.3 \\
\hline Neotropical Entomology & v. 33 , fisrt page 665 , last page 672 & $1519-566 X$ & 0.413 & 3.3 \\
\hline Neotropical Entomology & v. 33 , fisrt page 769 , last page 775 & $1519-566 x$ & 0.413 & 3.3 \\
\hline Neotropical Entomology & v. 33 , fisrt page 797 , last page 798 & $1519-566 x$ & 0.413 & 3.3 \\
\hline Neotropical Entomology & v. 34, fisrt page 1017, last page 1021 & $1519-566 x$ & 0.413 & 3.3 \\
\hline Neotropical Entomology & v. 34 , fisrt page 127, last page 129 & $1519-566 X$ & 0.413 & 3.3 \\
\hline Neotropical Entomology & v. 34 , fisrt page 163 , last page 168 & $1519-566 X$ & 0.413 & 3.3 \\
\hline Neotropical Entomology & v. 34 , fisrt page 169 , last page 175 & $1519-566 X$ & 0.413 & 3.3 \\
\hline Neotropical Entomology & v. 34 , fisrt page 177 , last page 181 & $1519-566 X$ & 0.413 & 3.3 \\
\hline Neotropical Entomology & v. 34 , fisrt page 183 , last page 190 & $1519-566 X$ & 0.413 & 3.3 \\
\hline Neotropical Entomology & v. 34 , fisrt page 191 , last page 201 & $1519-566 X$ & 0.413 & 3.3 \\
\hline Neotropical Entomology & v. 34 , fisrt page 215 , last page 219 & $1519-566 X$ & 0.413 & 3.3 \\
\hline Neotropical Entomology & v. 34 , fisrt page 227 , last page 233 & $1519-566 x$ & 0.413 & 3.3 \\
\hline Neotropical Entomology & v. 34 , fisrt page 251 , last page 254 & $1519-566 x$ & 0.413 & 3.3 \\
\hline Neotropical Entomology & v. 34 , fisrt page 281 , last page 289 & $1519-566 x$ & 0.413 & 3.3 \\
\hline Neotropical Entomology & v. 34 , fisrt page 325 , last page 329 & $1519-566 x$ & 0.413 & 3.3 \\
\hline Neotropical Entomology & v. 34 , fisrt page 331 , last page 336 & $1519-566 x$ & 0.413 & 3.3 \\
\hline Neotropical Entomology & v. 34 , fisrt page 341 , last page 347 & $1519-566 x$ & 0.413 & 3.3 \\
\hline Neotropical Entomology & v. 34 , fisrt page 357 , last page 362 & $1519-566 X$ & 0.413 & 3.3 \\
\hline
\end{tabular}


Neotropical Entomology

Neotropical Entomology

Neotropical Entomology

Neotropical Entomology

Neotropical Entomology

Neotropical Entomology

Neotropical Entomology

Neotropical Entomology

Neotropical Entomology

Neotropical Entomology

Neotropical Entomology

Neotropical Entomology

Neotropical Entomology

Neotropical Entomology

Neotropical Entomology

Neotropical Entomology

Neotropical Entomology

Neotropical Entomology

Neotropical Entomology

Neotropical Entomology

Neotropical Entomology

Neotropical Entomology

Neotropical Entomology

Neotropical Entomology

Neotropical Entomology

Neotropical Entomology

Neotropical Entomology

Neotropical Entomology

Neotropical Entomology

Neotropical Entomology

Neotropical Entomology

Neotropical Entomology

Neotropical Entomology

Neotropical Entomology

Neotropical Entomology

Neotropical Entomology

Neotropical Entomology

Neotropical Entomology

Neotropical Entomology

Neotropical Entomology

Neotropical Entomology

Neotropical Entomology

Neotropical Entomology

Neotropical Entomology

Neotropical Entomology

Neotropical Entomology

Neotropical Entomology

Neotropical Entomology

Neotropical Entomology

Neotropical Entomology

Neotropical Entomology

Neotropical Entomology

Neotropical Entomology

Neotropical Entomology

Neotropical Entomology

Neotropical Entomology

Neotropical Entomology

Neotropical Entomology

Neotropical Entomology

Neotropical Entomology
Paper info.

v. 34, fisrt page 363 , last page 374

v. 34 , fisrt page 375 , last page 380

v. 34 , fisrt page 387 , last page 394

v. 34 , fisrt page 403 , last page 406

v. 34 , fisrt page 443 , last page 450

v. 34 , fisrt page 475 , last page 484

v. 34 , fisrt page 515 , last page 517

v. 34, fisrt page 521, last page 524

v. 34, fisrt page 539, last page 545

v. 34 , fisrt page 547 , last page 554

v. 34 , fisrt page 571 , last page 576

v. 34 , fisrt page 585 , last page 591

v. 34 , fisrt page 601 , last page 606

v. 34 , fisrt page 63 , last page 66

v. 34 , fisrt page 733 , last page 741

v. 34 , fisrt page 769 , last page 776

v. 34 , fisrt page 791 , last page 798

v. 34 , fisrt page 829 , last page 836

v. 34 , fisrt page 837 , last page 841

v. 34 , fisrt page 849 , last page 851

v. 34 , fisrt page 881 , last page 888

v. 34, fisrt page 895, last page 902

v. 34 , fisrt page 911 , last page 915

v. 34, fisrt page 927, last page 932

v. 34, fisrt page 945 , last page 949

v. 34, fisrt page 999, last page 1006

v. 35 (1), fisrt page 141, last page 144

v. 35 (5), fisrt page 698, last page 704

v. 35 , fisrt page 1 , last page 4

v. 35 , fisrt page 12 , last page 18

v. 35 , fisrt page 120 , last page 125

v. 35 , fisrt page 149 , last page 151

v. 35 , fisrt page 175 , last page 181

v. 35 , fist page 21 , last page 29

v. 35 , fisrt page 273 , last page 276

v. 35 , fisrt page 277 , last page 278

v. 35 , fisrt page 279 , last page 281

v. 35 , fisrt page 338 , last page 342

v. 35 , fisrt page 344 , last page 348

v. 35 , fisrt page 357 , last page 363

v. 35 , fisrt page 371 , last page 376

v. 35, fisrt page 382 , last page 389

v. 35 , fisrt page 390 , last page 394

v. 35 , fisrt page 402 , last page 407

v. 35 , fisrt page 416 , last page 418

v. 35 , fisrt page 440 , last page 445

v. 35 , fisrt page 446 , last page 451

v. 35 , fisrt page 493 , last page 499

v. 35 , fisrt page 523 , last page 535

v. 35 , fisrt page 536 , last page 541

v. 35 , fisrt page 542 , last page 550

v. 35 , fisrt page 560 , last page 562

v. 35 , fisrt page 567 , last page 572

v. 35 , fisrt page 579 , last page 587

v. 35 , fisrt page 596, last page 601

v. 35, fisrt page 602 , last page 615

v. 35 , fisrt page 632 , last page 637

v. 35, fisrt page 654, last page 659

v. 35, fisrt page 660 , last page 665

v. 35 , fisrt page 666 , last page 670
ISSN

1519-566X

$1519-566 X$

$1519-566 X$

$1519-566 X$

1519-566X

1519-566X

1519-566X

1519-566X

1519-566X

1519-566X

1519-566X

1519-566X

1519-566X

1519-566X

1519-566X

1519-566X

1519-566X

1519-566X

1519-566X

1519-566X

1519-566X

1519-566X

1519-566X

1519-566X

1519-566X

1519-566X

1519-566X

1519-566X

1519-566X

1519-566X

1519-566X

1519-566X

1519-566X

1519-566X

1519-566X

1519-566X

1519-566X

1519-566X

1519-566X

1519-566X

1519-566X

1519-566X

1519-566X

1519-566X

1519-566X

1519-566X

1519-566X

1519-566X

1519-566X

1519-566X

1519-566X

1519-566X

1519-566X

1519-566X

1519-566X

1519-566X

1519-566X

1519-566X

1519-566X

1519-566X

IF Half-Life

$0.413 \quad 3.3$

$0.413 \quad 3.3$

$0.413 \quad 3.3$

$0.413 \quad 3.3$

$0.413 \quad 3.3$

$0.413 \quad 3.3$

$0.413 \quad 3.3$

$0.413 \quad 3.3$

$0.413 \quad 3.3$

$0.413 \quad 3.3$

$0.413 \quad 3.3$

$0.413 \quad 3.3$

$0.413 \quad 3.3$

$0.413 \quad 3.3$

$0.413 \quad 3.3$

$0.413 \quad 3.3$

$0.413 \quad 3.3$

$0.413 \quad 3.3$

$0.413 \quad 3.3$

$0.413 \quad 3.3$

$0.413 \quad 3.3$

$0.413 \quad 3.3$

$0.413 \quad 3.3$

$0.413 \quad 3.3$

$0.413 \quad 3.3$

$0.413 \quad 3.3$

$0.413 \quad 3.3$

$0.413 \quad 3.3$

$0.413 \quad 3.3$

$0.413 \quad 3.3$

$0.413 \quad 3.3$

$0.413 \quad 3.3$

$0.413 \quad 3.3$

$0.413 \quad 3.3$

$0.413 \quad 3.3$

$0.413 \quad 3.3$

$0.413 \quad 3.3$

$0.413 \quad 3.3$

$0.413 \quad 3.3$

$0.413 \quad 3.3$

$0.413 \quad 3.3$

$0.413 \quad 3.3$

$0.413 \quad 3.3$

$0.413 \quad 3.3$

$0.413 \quad 3.3$

$0.413 \quad 3.3$

$0.413 \quad 3.3$

$0.413 \quad 3.3$

$0.413 \quad 3.3$

$0.413 \quad 3.3$

$0.413 \quad 3.3$

$0.413 \quad 3.3$

$0.413 \quad 3.3$

$0.413 \quad 3.3$

$0.413 \quad 3.3$

$0.413 \quad 3.3$

$0.413 \quad 3.3$

$0.413 \quad 3.3$

$0.413 \quad 3.3$

$0.413 \quad 3.3$ 
Neotropical Entomology

Neotropical Entomology

Neotropical Entomology

Neotropical Entomology

Neotropical Entomology

Neotropical Entomology

Neotropical Entomology

Neotropical Entomology

Neotropical Entomology

Neotropical Entomology

Neotropical Entomology

Seed Science and Technology

Seed Science and Technology

Seed Science and Technology

Seed Science and Technology

Seed Science and Technology

Deutsche Entomologische Zeitschrift

International Journal of Environment and

Pollution

Vie et Milieu

American Fern Journal

American Fern Journal

American Fern Journal

Caribbean Journal of Science

Caribbean Journal of Science

Coleopterists Bulletin

Pharmaceutical Biology

Pharmaceutical Biology

Folia Biologica

Folia Biologica

Revista Brasileira de Zootecnia / Brazilian Journal of Animal Science

Revista Brasileira de Zootecnia / Brazilian Journal of Animal Science

Revista Brasileira de Zootecnia / Brazilian Journal of Animal Science

Revista Brasileira de Zootecnia / Brazilian Journal of Animal Science

Revista Brasileira de Zootecnia / Brazilian Journal of Animal Science

Revista Brasileira de Zootecnia / Brazilian Journal of Animal Science

Revista Brasileira de Zootecnia / Brazilian Journal of Animal Science

Revista Brasileira de Zootecnia / Brazilian Journal of Animal Science

Brazilian Journal of Chemical Engineering

Journal of Equine Veterinary Science

Proceedings of the Biological Society of Washington

Proceedings of the Biological Society of Washington

Proceedings of the Biological Society of Washington

Mammalia (Paris)

Mammalia (Paris)

Mammalia (Paris)

Mammalia (Paris)

Mammalia (Paris)

Mammalia (Paris)

Mammalia (Paris)

Mammalia (Paris)

Mammalia (Paris)

Journal de Physique. IV

Journal of Essential Oil Research
Paper info.

ISSN

v. 35, fisrt page 689 , last page 697

v. 35 , fisrt page 698 , last page 704

v. 35 , fisrt page 707 , last page 709

v. 35 , fisrt page 724 , last page 730

v. 35 , fisrt page 747 , last page 752

v. 35 , fisrt page 753 , last page 756

v. 35 , fisrt page 757 , last page 761

v. 35 , fisrt page 762 , last page 768

v. 35 , fisrt page 775 , last page 780

v. 35 , fisrt page 868 , last page 870

v. 36 , fisrt page 828 , last page 833

v. 32 , fisrt page 119 , last page 134

v. 32 , fisrt page 759 , last page 764

v. 33 , fisrt page 105 , last page 113

v. 33 , fisrt page 147 , last page 156

v. 34, fisrt page 585, last page 592

v. 53 , fisrt page 108 , last page 113

v. 21 , fisrt page 153 , last page 165

v. 55 , fist page 71 , last page 79

v. 94 , fisrt page 112 , last page 114

v. 95 , fisrt page 115 , last page 125

v. 95 , fisrt page 89 , last page 93

v. 41 , fisrt page 600 , last page 612

v. 41 , fisrt page 638 , last page 703

v. 59 , fisrt page 339 , last page 350

v. 42 , fisrt page 565 , last page 569

v. 43 , fisrt page 480 , last page 484

v. 51 , fisrt page 40 , last page 46

v. 51 , fist page 68 , last page 75

v. 32 , fist page 1835, last page 1844

v. 33 , n. supl. 2, fisrt page 1928, last page 1933

v. 33 , fisrt page 1 , last page 7

v. 33, fisrt page 1934, last page 1941

v. 33, fisrt page 519 , last page 528

v. 33 , fisrt page 821 , last page 827

v. 34, fisrt page 907 , last page 914

v. 35, fisrt page 1893, last page 1896

v. 22 , fisrt page 489 , last page 493

v. 24, fisrt page 204, last page 209

v. 117 , fisrt page 317 , last page 329

v. 118 , fisrt page 855 , last page 862

v. 119 , fisrt page 395 , last page 403

v. 67 , fisrt page 503 , last page 511

v. 68 , fisrt page 233 , last page 237

v. 68 , fisrt page 27 , last page 36

v. 68 , fisrt page 5 , last page 15

v. 69 , fisrt page 245 , last page 250

v. 69 , fisrt page 417 , last page 419

v. 69 , fisrt page 427 , last page 430

v. 69 , fisrt page 431 , last page 434

v. 70 , fisrt page 52 , last page 57

v. 125 , fisrt page 877 , last page 879

v. 16 , fist page 15 , last page 17
1519-566X

1519-566X

$1519-566 X$

$1519-566 X$

$1519-566 X$

$1519-566 X$

1519-566X

1519-566X

1519-566X

$1519-566 X$

$1519-566 \mathrm{X}$

0251-0952

0251-0952

0251-0952

0251-0952

0251-0952

0012-0073

0957-4352

0240-8759

0002-8444

0002-8444

0002-8444

0008-6452

0008-6452

0010-065X

1388-0209

1388-0209

0015-5500

0015-5500

1516-3598

1516-3598

1516-3598

1516-3598

1516-3598

1516-3598

1516-3598

1516-3598

0104-6632

0737-0806

0006-324X

0006-324X

0006-324X

0025-1461

0025-1461

0025-1461

0025-1461

0025-1461

0025-1461

0025-1461

0025-1461

0025-1461

1155-4339

1041-2905

IF Half-Life

$0.413 \quad 3.3$

$0.413 \quad 3.3$

$0.413 \quad 3.3$

$0.413 \quad 3.3$

$0.413 \quad 3.3$

$0.413 \quad 3.3$

$0.413 \quad 3.3$

$0.413 \quad 3.3$

$0.413 \quad 3.3$

$0.413 \quad 3.3$

$0.413 \quad 3.3$

$0.41 \quad 10$

$0.41 \quad 10$

$0.41 \quad 10$

$0.41 \quad 10$

$0.41 \quad 10$

$0.407 \quad 10$

$0.404 \quad 6$

$0.404 \quad 10$

$0.4 \quad 10$

$0.4 \quad 10$

$0.4 \quad 10$

$0.4 \quad 8.8$

$0.4 \quad 8.8$

$0.397 \quad 10$

$0.397 \quad 4.4$

$0.397 \quad 4.4$

$0.387 \quad 7.6$

$0.387 \quad 7.6$

$0.385 \quad 5.2$

$0.385 \quad 5.2$

$0.385 \quad 5.2$

$0.385 \quad 5.2$

$0.385 \quad 5.2$

$0.385 \quad 5.2$

$0.385 \quad 5.2$

$0.385 \quad 5.2$

$0.377 \quad 5.2$

$0.366 \quad 10$

$0.347 \quad 10$

$0.347 \quad 10$

$0.347 \quad 10$

$0.333 \quad 10$

$0.333 \quad 10$

$0.333 \quad 10$

$0.333 \quad 10$

$0.333 \quad 10$

$0.333 \quad 10$

$0.333 \quad 10$

$0.333 \quad 10$

$0.333 \quad 10$

$0.315 \quad 6$

$0.309 \quad 7.8$ 
Journal of Essential Oil Research Journal of Essential Oil Research Journal of Essential Oil Research Journal of Essential Oil Research Journal of Essential Oil Research Journal of Essential Oil Research Journal of Essential Oil Research Journal of Essential Oil Research Journal of Essential Oil Research Journal of Essential Oil Research Journal of Essential Oil Research Scientia Agricola

Scientia Agricola

Scientia Agricola

Scientia Agricola

Scientia Agricola

Scientia Agricola

Scientia Agricola

Scientia Agricola

Scientia Agricola

Scientia Agricola

Scientia Agricola

Scientia Agricola

Scientia Agricola

Scientia Agricola

Biological Rhythm Research

Biological Rhythm Research

Biological Rhythm Research

Journal of Conchology

Pesquisa Agropecuária Brasileira

Pesquisa Agropecuária Brasileira

Pesquisa Agropecuária Brasileira

Pesquisa Agropecuária Brasileira

Pesquisa Agropecuária Brasileira

Pesquisa Agropecuária Brasileira

Pesquisa Agropecuária Brasileira

Pesquisa Agropecuária Brasileira

Pesquisa Agropecuária Brasileira

Pesquisa Agropecuária Brasileira

Pesquisa Agropecuária Brasileira

Pesquisa Agropecuária Brasileira

Pesquisa Agropecuária Brasileira

Pesquisa Agropecuária Brasileira

Pesquisa Agropecuária Brasileira

Pesquisa Agropecuária Brasileira

Pesquisa Agropecuária Brasileira

Pesquisa Agropecuária Brasileira

Pesquisa Agropecuária Brasileira

Pesquisa Agropecuária Brasileira

Pesquisa Agropecuária Brasileira

Pesquisa Agropecuária Brasileira

Pesquisa Agropecuária Brasileira

Pesquisa Agropecuária Brasileira

Pesquisa Agropecuária Brasileira

Pesquisa Agropecuária Brasileira

Pesquisa Agropecuária Brasileira

Pesquisa Agropecuária Brasileira

Pesquisa Agropecuária Brasileira

Pesquisa Agropecuária Brasileira

Pesquisa Agropecuária Brasileira
Paper info.

v. 16, fisrt page 405, last page 408

v. 17 , fisrt page 209 , last page 211

v. 17 , fisrt page 455 , last page 456

v. 18 , fisrt page 10 , last page 12

v. 18 , fisrt page 142 , last page 145

v. 18 , fisrt page 269 , last page 271

v. 18 , fisrt page 345 , last page 347

v. 18, fisrt page 392, last page 395

v. 18 , fist page 60 , last page 63

v. 18 , fisrt page 61 , last page 65

v. 7, fisrt page 151, last page 154

v. 01 , fisrt page 17 , last page 20

v. 1, fisrt page 353 , last page 357

v. 3, fisrt page 296, last page 298

v. 61 , fisrt page 298 , last page 302

v. 61 , fisrt page 462 , last page 465

v. 61 , fisrt page 82 , last page 87

v. 62 , fisrt page 227 , last page 234

v. 62 , fisrt page 406 , last page 412

v. 62 , fisrt page 72 , last page 75

v. 63 , fisrt page 40 , last page 45

v. 63 , fisrt page 433 , last page 438

v. 63 , fisrt page 478 , last page 482

v. 63 , fisrt page 567 , last page 571

v. 65 , fisrt page 439 , last page 443

v. 35 , fisrt page 1 , last page 3

v. 35 , fisrt page 159 , last page 169

v. 36 , fisrt page 229 , last page 236

v. 39 , fisrt page 1 , last page 4

v. 38 , fisrt page 763 , last page 770

v. 39, fisrt page 1027, last page 1032

v. 39, fisrt page 1087, last page 1093

v. 39 , fisrt page 113 , last page 121

v. 39 , fisrt page 193, last page 196

v. 39, fisrt page 197, last page 199

v. 39 , fisrt page 247 , last page 253

v. 39 , fisrt page 421 , last page 430

v. 39 , fisrt page 525 , last page 531

v. 39, fisrt page 615 , last page 623

v. 39, fisrt page 637 , last page 643

v. 39 , fisrt page 735 , last page 740

v. 39, fisrt page 757 , last page 762

v. 39 , fisrt page 841 , last page 845

v. 39 , fisrt page 947 , last page 951

v. 40, fisrt page 1141, last page 1144

v. 40 , fisrt page 217 , last page 223

v. 40 , fisrt page 225 , last page 232

v. 40 , fisrt page 323 , last page 328

v. 40 , fisrt page 345 , last page 351

v. 40 , fisrt page 447 , last page 452

v. 40 , fisrt page 613 , last page 616

v. 40 , fisrt page 723 , last page 726

v. 40 , fisrt page 895 , last page 898

v. 40 , fisrt page 911 , last page 917

v. 41, fisrt page 1285, last page 1290

v. 41, fisrt page 165, last page 169

v. 41, fist page 1699, last page 1705

v. 41 , fist page 1739, last page 1748

v. 41 , fisrt page 185 , last page 194

v. 41 , fisrt page 195, last page 203
ISSN

1041-2905

1041-2905

1041-2905

1041-2905

1041-2905

1041-2905

1041-2905

1041-2905

1041-2905

1041-2905

1041-2905

0103-9016

0103-9016

0103-9016

0103-9016

0103-9016

0103-9016

0103-9016

0103-9016

0103-9016

0103-9016

0103-9016

0103-9016

0103-9016

0103-9016

0929-1016

0929-1016

0929-1016

0022-0019

0100-204X

0100-204X

0100-204X

0100-204X

0100-204X

0100-204X

0100-204X

0100-204X

0100-204X

0100-204X

0100-204X

0100-204X

0100-204X

0100-204X

0100-204X

0100-204X

0100-204X

0100-204X

0100-204X

0100-204X

0100-204X

0100-204X

0100-204X

0100-204X

0100-204X

0100-204X

0100-204X

0100-204X

0100-204X

0100-204X

0100-204X
Half-Life

$0.309 \quad 7.8$

$0.309 \quad 7.8$

$0.309 \quad 7.8$

$0.309 \quad 7.8$

$0.309 \quad 7.8$

$0.309 \quad 7.8$

$0.309 \quad 7.8$

$0.309 \quad 7.8$

$0.309 \quad 7.8$

$\begin{array}{ll}0.309 & 7.8\end{array}$

$0.309 \quad 7.8$

$0.298 \quad 6.1$

$0.298 \quad 6.1$

$0.298 \quad 6.1$

$0.298 \quad 6.1$

$0.298 \quad 6.1$

$0.298 \quad 6.1$

$0.298 \quad 6.1$

$0.298 \quad 6.1$

$0.298 \quad 6.1$

$0.298 \quad 6.1$

$0.298 \quad 6.1$

$0.298 \quad 6.1$

$0.298 \quad 6.1$

$0.298 \quad 6.1$

$0.296 \quad 6.1$

$0.296 \quad 6.1$

$0.296 \quad 6.1$

$0.292 \quad 10$

$0.286 \quad 7$

$0.286 \quad 7$

$0.286 \quad 7$

$0.286 \quad 7$

$0.286 \quad 7$

$0.286 \quad 7$

$0.286 \quad 7$

$0.286 \quad 7$

$0.286 \quad 7$

$0.286 \quad 7$

$0.286 \quad 7$

$0.286 \quad 7$

$0.286 \quad 7$

$0.286 \quad 7$

$0.286 \quad 7$

$0.286 \quad 7$

$0.286 \quad 7$

$0.286 \quad 7$

$0.286 \quad 7$

$0.286 \quad 7$

$0.286 \quad 7$

$0.286 \quad 7$

$0.286 \quad 7$

$0.286 \quad 7$

$0.286 \quad 7$

$0.286 \quad 7$

$0.286 \quad 7$

$0.286 \quad 7$

$0.286 \quad 7$

$0.286 \quad 7$

$0.286 \quad 7$ 


\begin{tabular}{|c|c|c|c|c|}
\hline Journal & Paper info. & ISSN & IF & Half-Life \\
\hline Pesquisa Agropecuária Brasileira & v. 41 , fistt page 223 , last page 232 & $0100-204 X$ & 0.286 & 7 \\
\hline Pesquisa Agropecuária Brasileira & v. 41 , fistt page 275 , last page 284 & 0100-204X & 0.286 & 7 \\
\hline Pesquisa Agropecuária Brasileira & v. 41 , fisrt page 31 , last page 35 & $0100-204 X$ & 0.286 & 7 \\
\hline Pesquisa Agropecuária Brasileira & v. 41 , fisrt page 385 , last page 391 & $0100-204 X$ & 0.286 & 7 \\
\hline Pesquisa Agropecuária Brasileira & v. 41 , fisrt page 393 , last page 398 & $0100-204 X$ & 0.286 & 7 \\
\hline Pesquisa Agropecuária Brasileira & v. 41 , fisrt page 439 , last page 447 & $0100-204 X$ & 0.286 & 7 \\
\hline Pesquisa Agropecuária Brasileira & v. 41 , fistt page 517 , last page 523 & $0100-204 X$ & 0.286 & 7 \\
\hline Pesquisa Agropecuária Brasileira & v. 41 , fisrt page 811 , last page 818 & $0100-204 X$ & 0.286 & 7 \\
\hline Pesquisa Agropecuária Brasileira & v. 41 , fisrt page 873 , last page 877 & $0100-204 X$ & 0.286 & 7 \\
\hline Pesquisa Agropecuária Brasileira (Online) & v. 40 , fisrt page 947 , last page 951 & $1678-3921$ & 0.286 & 7 \\
\hline Pesquisa Agropecuária Brasileira, Brasilia & v. 41 , fistt page 85 , last page 92 & 0100-204X & 0.286 & 7 \\
\hline Revista Brasileira de Ciência do Solo & v. 28 , fisrt page 289 , last page 295 & 0100-0683 & 0.286 & 3.3 \\
\hline Revista Brasileira de Ciência do Solo & v. 29 , fistt page 373 , last page 378 & $0100-0683$ & 0.286 & 3.3 \\
\hline Revista Brasileira de Ciência do Solo & v. 30 , fistt page 281 , last page 291 & $0100-0683$ & 0.286 & 3.3 \\
\hline Revista Brasileira de Ciência do Solo & v. 30 , fisrt page 401 , last page 412 & 0100-0683 & 0.286 & 3.3 \\
\hline Revista Brasileira de Ciência do Solo & v. 30 , fisrt page 555 , last page 564 & 0100-0683 & 0.286 & 3.3 \\
\hline Revista Brasileira de Ciência do Solo & v. 31 , fistt page 46 , last page 50 & 0100-0683 & 0.286 & 3.3 \\
\hline Odonatologica & v. 33 , fisrt page 17 , last page 25 & 0375-0183 & 0.284 & 10 \\
\hline Odonatologica & v. 33, fisrt page 25 , last page 39 & 0375-0183 & 0.284 & 10 \\
\hline Odonatologica & v. 34, fisrt page 107, last page 112 & $0375-0183$ & 0.284 & 10 \\
\hline Odonatologica & v. 34, fisrt page 35, last page 49 & $0375-0183$ & 0.284 & 10 \\
\hline Nautilus (Philadelphia) & v. 118 , fistt page 160 , last page 167 & $0028-1344$ & 0.278 & 10 \\
\hline Nautilus (Philadelphia) & v. 119 , fist page 169 , last page 173 & $0028-1344$ & 0.278 & 10 \\
\hline Caryologia (Firenze) & v. 38, fisrt page 70, last page 77 & $0008-7114$ & 0.261 & 10 \\
\hline Caryologia (Firenze) & v. 57 , fisrt page 1 , last page 3 & $0008-7114$ & 0.261 & 10 \\
\hline Caryologia (Firenze) & v. 57, fisrt page 1, last page 9 & $0008-7114$ & 0.261 & 10 \\
\hline Caryologia (Firenze) & v. 57 , fisrt page 183, last page 187 & $0008-7114$ & 0.261 & 10 \\
\hline Caryologia (Firenze) & v. 57 , fisrt page 219 , last page 223 & $0008-7114$ & 0.261 & 10 \\
\hline Caryologia (Firenze) & v. 57 , fisrt page 250 , last page 258 & $0008-7114$ & 0.261 & 10 \\
\hline Caryologia (Firenze) & v. 57, fisrt page 31, last page 37 & $0008-7114$ & 0.261 & 10 \\
\hline Caryologia (Firenze) & v. 57 , fisrt page 53 , last page 65 & $0008-7114$ & 0.261 & 10 \\
\hline Caryologia (Firenze) & v. 57 , fist page 69 , last page 73 & $0008-7114$ & 0.261 & 10 \\
\hline Caryologia (Firenze) & v. 57 , fisrt page 89 , last page 97 & $0008-7114$ & 0.261 & 10 \\
\hline Caryologia (Firenze) & v. 58, fisrt page 21, last page 27 & $0008-7114$ & 0.261 & 10 \\
\hline Caryologia (Firenze) & v. 58 , fisrt page 249 , last page 254 & $0008-7114$ & 0.261 & 10 \\
\hline Caryologia (Firenze) & v. 58 , fisrt page 293 , last page 299 & $0008-7114$ & 0.261 & 10 \\
\hline Caryologia (Firenze) & v. 59 , fisrt page 14 , last page 18 & $0008-7114$ & 0.261 & 10 \\
\hline Caryologia (Firenze) & v. 59 , fisrt page 43 , last page 46 & $0008-7114$ & 0.261 & 10 \\
\hline Caryologia (Firenze) & v. 59 , fisrt page 7, last page 13 & $0008-7114$ & 0.261 & 10 \\
\hline Caryologia (Firenze) & v. n. 2, fisrt page 102, last page 111 & $0008-7114$ & 0.261 & 10 \\
\hline Ornitología Neotropical & v. 1, fisrt page 75, last page 92 & $1075-4377$ & 0.26 & 4.1 \\
\hline Ornitología Neotropical & v. 15 , fisrt page 117 , last page 126 & $1075-4377$ & 0.26 & 4.1 \\
\hline Ornitología Neotropical & v. 15 , fisrt page 137 , last page 144 & $1075-4377$ & 0.26 & 4.1 \\
\hline Ornitología Neotropical & v. 15 , fisrt page 527 , last page 538 & $1075-4377$ & 0.26 & 4.1 \\
\hline Ornitología Neotropical & v. 15, fisrt page 73, last page 83 & $1075-4377$ & 0.26 & 4.1 \\
\hline Ornitología Neotropical & v. 16 , fisrt page 253 , last page 262 & $1075-4377$ & 0.26 & 4.1 \\
\hline Ornitología Neotropical & v. 16 , fisrt page 471 , last page 479 & $1075-4377$ & 0.26 & 4.1 \\
\hline Ornitología Neotropical & v. 17 , fisrt page 213 , last page 225 & $1075-4377$ & 0.26 & 4.1 \\
\hline Ornitología Neotropical & v. 17 , fisrt page 363 , last page 371 & $1075-4377$ & 0.26 & 4.1 \\
\hline Ornitología Neotropical & v. 17 , fisrt page 481 , last page 490 & $1075-4377$ & 0.26 & 4.1 \\
\hline $\begin{array}{l}\text { Brazilian Archives of Biology and } \\
\text { Technology }\end{array}$ & v. 4 , fisrt page 643 , last page 650 & $1516-8913$ & 0.225 & 4.1 \\
\hline $\begin{array}{l}\text { Brazilian Archives of Biology and } \\
\text { Technology }\end{array}$ & v. 47 , fisrt page 1 , last page 14 & $1516-8913$ & 0.225 & 4.1 \\
\hline Brazilian Archives of Biology and & v. 47 , fisrt page 127 , last page 133 & $1516-8913$ & & \\
\hline $\begin{array}{l}\text { Technology } \\
\text { Brazilian Archives of Biology and }\end{array}$ & v. 47 , fisrt page 153 , last page 162 & $1516-8913$ & 0.225 & 4.1 \\
\hline Technology & & & 0.225 & 4.1 \\
\hline $\begin{array}{l}\text { Brazilian Archives of Biology and } \\
\text { Technology }\end{array}$ & v. 47 , fisrt page 185 , last page 191 & $1516-8913$ & 0.225 & 4.1 \\
\hline $\begin{array}{l}\text { Brazilian Archives of Biology and } \\
\text { Technology }\end{array}$ & v. 47 , fisrt page 273 , last page 279 & $1516-8913$ & 0.225 & 4.1 \\
\hline
\end{tabular}


Journal

Paper info.

ISSN

Brazilian Archives of Biology and

v. 47, fisrt page 291, last page 299

$1516-8913$

Technology

v. 47, fisrt page 355, last page 362

0.225

Half-Life

Brazilian Archives of Biology and

$1516-8913$

Technology

v. 47 , fisrt page 363 , last page 373

0.225

4.1

Brazilian Archives of Biology and

v. 47 , fisrt page 423 , last page 431

0.225

4.1

Brazilian Archives of Biology and

Technology

Brazilian Archives of Biology and

Technology

Brazilian Archives of Biology and

Technology

Brazilian Archives of Biology and

Technology

Brazilian Archives of Biology and

Technology

Brazilian Archives of Biology and

Technology

Brazilian Archives of Biology and

Technology

Brazilian Archives of Biology and

Technology

Brazilian Archives of Biology and

Technology

Brazilian Archives of Biology and

Technology

Brazilian Archives of Biology and

Technology

Brazilian Archives of Biology and

Technology

Brazilian Archives of Biology and

Technology

Brazilian Archives of Biology and

Technology

Brazilian Archives of Biology and

Technology

Brazilian Archives of Biology and

Technology

Brazilian Archives of Biology and

Technology

Brazilian Archives of Biology and

Technology

Brazilian Archives of Biology and

Technology

Brazilian Archives of Biology and

Technology

Brazilian Archives of Biology and

Technology

Brazilian Archives of Biology and

Technology

Brazilian Archives of Biology and

Technology

Brazilian Archives of Biology and

Technology

Brazilian Archives of Biology and

Technology

Brazilian Archives of Biology and

Technology

Brazilian Archives of Biology and

Technology

Brazilian Archives of Biology and

Technology

Brazilian Archives of Biology and

Technology

Brazilian Archives of Biology and

Technology

Brazilian Archives of Biology and

Technology

Brazilian Archives of Biology and

47, fisrt page 451, last page 459

v. 47 , fisrt page 461 , last page 467

v. 47 , fisrt page 485 , last page 493

$1516-8913$

$1516-8913$

0.225

$1516-8913$

0.225

$1516-8913$

0.225

$1516-8913$

v. 47 , fisrt page 495 , last page 501

0.225

$1516-8913$

v. 47 , fisrt page 50 , last page 61

0.225

4.1

$1516-8913$

v. 47 , fisrt page 511 , last page 520

0.225

4.1

$1516-8913$

v. 47 , fisrt page 531, last page 535

0.225

$1516-8913$

v. 47 , fisrt page 575 , last page 577

0.225

$1516-8913$

v. 47 , fisrt page 601 , last page 612

0.225

$1516-8913$

v. 47 , fisrt page 613 , last page 627

0.225

$1516-8913$

v. 47 , fisrt page 635 , last page 641

0.225

$1516-8913$

v. 47 , fisrt page 685 , last page 691

0.225

4.1

$1516-8913$

v. 47 , fisrt page 713 , last page 723

0.225

4.1

$1516-8913$

v. 47 , fisrt page 739 , last page 745

0.225

$1516-8913$

v. 47 , fisrt page 755 , last page 763

0.225

$1516-8913$

v. 47 , fisrt page 765 , last page 774

0.225

$1516-8913$

v. 47 , fisrt page 775 , last page 783

0.225

$1516-8913$

v. 47 , fisrt page 785 , last page 792

0.225

$1516-8913$

v. 47 , fisrt page 799 , last page 804

0.225

$1516-8913$

v. 47 , fisrt page 805 , last page 811

0.225

$1516-8913$

v. 47 , fisrt page 821 , last page 826

0.225

$1516-8913$

v. 47 , fisrt page 873 , last page 879

$1516-8913$

v. 47 , fisrt page 887 , last page 894

0.225

$1516-8913$

v. 47 , fisrt page 933 , last page 943

0.225

$1516-8913$

v. 47 , fisrt page 961 , last page 971

0.225

$1516-8913$

v. 48 , fisrt page 1013, last page 1026

0.225

$1516-8913$

v. 48 , fisrt page 109 , last page 113

0.225

$1516-8913$

v. 48 , fisrt page 113 , last page 117

0.225

v. 48 , fisrt page 115 , last page 119

v. 48 , fisrt page 147 , last page 153

0.225

v. 48 , fisrt page 171 , last page 180

0.225

v. 48 , fisrt page 181 , last page 185

Brazilian Archives of Biology and

Technology

Brazilian Archives of Biology and

Technology

v. 48 , fisrt page 217 , last page 225

$1516-8913$ 
Journal

Paper info.

ISSN

Brazilian Archives of Biology and

v. 48 , fisrt page 267 , last page 274

$1516-8913$

Technology

v. 48 , fisrt page 343 , last page 350

0.225

Half-Life

Brazilian Archives of Biology and

$1516-8913$

Technology

v. 48 , fisrt page 419 , last page 427

0.225

4.1

Brazilian Archives of Biology and

v. 48 , fisrt page 473 , last page 482

0.225

4.1

Brazilian Archives of Biology and

Technology

Brazilian Archives of Biology and

Technology

Brazilian Archives of Biology and

Technology

Brazilian Archives of Biology and

Technology

Brazilian Archives of Biology and

Technology

Brazilian Archives of Biology and

Technology

Brazilian Archives of Biology and

Technology

Brazilian Archives of Biology and

Technology

Brazilian Archives of Biology and

Technology

Brazilian Archives of Biology and

Technology

Brazilian Archives of Biology and

Technology

Brazilian Archives of Biology and

Technology

Brazilian Archives of Biology and

Technology

Brazilian Archives of Biology and

Technology

Brazilian Archives of Biology and

Technology

Brazilian Archives of Biology and

Technology

Brazilian Archives of Biology and

Technology

Brazilian Archives of Biology and

Technology

Brazilian Archives of Biology and

Technology

Brazilian Archives of Biology and

Technology

Brazilian Archives of Biology and

Technology

Brazilian Archives of Biology and

Technology

Brazilian Archives of Biology and

Technology

Brazilian Archives of Biology and

Technology

Brazilian Archives of Biology and

Technology

Brazilian Archives of Biology and

Technology

Brazilian Archives of Biology and

Technology

Brazilian Archives of Biology and

Technology

Brazilian Archives of Biology and

Technology

Brazilian Archives of Biology and

Technology

Brazilian Archives of Biology and

Technology

Brazilian Archives of Biology and

48, fisrt page 581 , last page 587

0.225

4.1

$1516-8913$

$1516-8913$

v. 48 , fisrt page 589 , last page 597

0.225

4.1

$1516-8913$

v. 48 , fisrt page 599 , last page 610

0.225

$1516-8913$

v. 48 , fisrt page 787 , last page 800

0.225

$1516-8913$

v. 48 , fisrt page 807 , last page 814

0.225

4.1

$1516-8913$

v. 48 , fisrt page 81 , last page 90

0.225

4.1

$1516-8913$

v. 48 , fisrt page 851 , last page 861

0.225

4.1

$1516-8913$

v. 48 , fisrt page 911 , last page 919

0.225

$1516-8913$

v. 48 , fisrt page 947 , last page 950

0.225

$1516-8913$

v. 48 , fisrt page 951 , last page 965

0.225

$1516-8913$

v. 48 , fisrt page 96 , last page 98

0.225

$1516-8913$

v. 49 , fisrt page 117 , last page 123

0.225

4.1

$1516-8913$

v. 49 , fisrt page 135 , last page 144

0.225

4.1

$1516-8913$

v. 49 , fisrt page 139 , last page 144

0.225

$1516-8913$

v. 49 , fisrt page 163, last page 169

$1516-8913$

v. 49 , fisrt page 171, last page 182

0.225

$1516-8913$

v. 49 , fisrt page 189 , last page 196

0.225

$1516-8913$

v. 49 , fisrt page 231 , last page 238

0.225

$1516-8913$

v. 49 , fisrt page 239 , last page 248

0.225

$1516-8913$

v. 49 , fisrt page 257 , last page 261

0.225

4.1

$1516-8913$

v. 49 , fisrt page 29 , last page 36

v. 49 , fisrt page 323 , last page 337

v. 49 , fisrt page 339 , last page 345

$1516-8913$

0.225

$1516-8913$

0.225

$1516-8913$

0.225

v. 49 , fisrt page 457 , last page 461

0.225

v. 49 , fisrt page 463 , last page 473

0.225

$1516-8913$

v. 49 , fisrt page 49 , last page 55

0.225

$1516-8913$

v. 49 , fisrt page 527 , last page 535

0.225

v. 49 , fisrt page 547 , last page 555

0.225

v. 49 , fisrt page 599, last page 604

v. 49 , fisrt page 73 , last page 77

0.225

v. 49 , fisrt page 763 , last page 774

0.225

v. 49 , fisrt page 807 , last page 812

Brazilian Archives of Biology and

Technology

Brazilian Archives of Biology and

Technology

v. 49 , fisrt page 905 , last page 913

1516-8913 


\begin{tabular}{|c|c|c|c|c|}
\hline Journal & Paper info. & ISSN & $\mathrm{IF}$ & Half-Life \\
\hline Brazilian Archives of Biology and & v. 49, fisrt page 915 , last page 921 & $1516-8913$ & & \\
\hline $\begin{array}{l}\text { Technology } \\
\text { Brazilian Archives of Biology and }\end{array}$ & v. 49, fisrt page 923 , last page 926 & $1516-8913$ & 0.225 & 4.1 \\
\hline 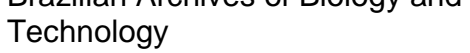 & & & 0.225 & 4.1 \\
\hline Brazilian Archives of Biology and & v. 49 , fisrt page 927 , last page 934 & $1516-8913$ & 0225 & 41 \\
\hline Brazilian Archives of Biology and & v. 49 , fisrt page 935 , last page 944 & $1516-8913$ & & \\
\hline Technology & & & 0.225 & 4.1 \\
\hline $\begin{array}{l}\text { Brazilian Archives of Biology and } \\
\text { Technology }\end{array}$ & v. 49 , fisrt page 945 , last page 951 & $1516-8913$ & 0.225 & 4.1 \\
\hline Brazilian Archives of Biology and & v. 49 , fisrt page 975 , last page 980 & $1516-8913$ & & \\
\hline Technology & & & 0.225 & 4.1 \\
\hline Brazilian Archives of Biology and & v. 62 , fisrt page 147 , last page 152 & $1516-8913$ & & \\
\hline Technology & & & 0.225 & 4.1 \\
\hline $\begin{array}{l}\text { Brazilian Archives of Biology and } \\
\text { Technology, TECPAR }\end{array}$ & v. 47 , fisrt page 339 , last page 346 & $1516-8913$ & 0.225 & 4.1 \\
\hline Interciencia (Caracas) & v. 29 , fisrt page 219 , last page 222 & 0378-1844 & 0.218 & 5.9 \\
\hline Interciencia (Caracas) & v. 30 , fisrt page 764 , last page 769 & 0378-1844 & 0.218 & 5.9 \\
\hline Interciencia (Caracas) & v. 31 , fisrt page 123 , last page 129 & 0378-1844 & 0.218 & 5.9 \\
\hline Interciencia (Caracas) & v. 31 , fisrt page 512 , last page 516 & 0378-1844 & 0.218 & 5.9 \\
\hline Revista de Biologia Tropical & v. 52 , fisrt page 109 , last page 114 & $0034-7744$ & 0.217 & 9.1 \\
\hline Revista de Biologia Tropical & v. 52 , fisrt page 143 , last page 156 & $0034-7744$ & 0.217 & 9.1 \\
\hline Revista de Biologia Tropical & v. 52 , fisrt page 387 , last page 392 & $0034-7744$ & 0.217 & 9.1 \\
\hline Revista de Biología Tropical & v. 52, fisrt page 47 , last page 55 & $0034-7744$ & 0.217 & 9.1 \\
\hline Revista de Biología Tropical & v. 53 , fisrt page 175 , last page 186 & $0034-7744$ & 0.217 & 9.1 \\
\hline Revista de Biología Tropical & v. 53, fisrt page 305 , last page 312 & $0034-7744$ & 0.217 & 9.1 \\
\hline Revista de Biología Tropical & v. 54, fisrt page 1171 , last page 1178 & $0034-7744$ & 0.217 & 9.1 \\
\hline Revista de Biología Tropical & v. 54, fisrt page 1197, last page 1203 & $0034-7744$ & 0.217 & 9.1 \\
\hline Revista de Biología Tropical & v. 54, fisrt page 943 , last page 950 & $0034-7744$ & 0.217 & 9.1 \\
\hline Brazilian Journal of Microbiology & v. 35 , fisrt page 126 , last page 130 & $1517-8382$ & 0.213 & 4.5 \\
\hline Brazilian Journal of Microbiology & v. 35 , fisrt page 205 , last page 210 & $1517-8382$ & 0.213 & 4.5 \\
\hline Brazilian Journal of Microbiology & v. 35 , fisrt page 219 , last page 223 & $1517-8382$ & 0.213 & 4.5 \\
\hline Brazilian Journal of Microbiology & v. 35 , fisrt page 243 , last page 247 & $1517-8382$ & 0.213 & 4.5 \\
\hline Brazilian Journal of Microbiology & v. 35 , fisrt page 275 , last page 280 & $1517-8382$ & 0.213 & 4.5 \\
\hline Brazilian Journal of Microbiology & v. 35 , fisrt page 295, last page 299 & $1517-8382$ & 0.213 & 4.5 \\
\hline Brazilian Journal of Microbiology & v. 35 , fisrt page 324 , last page 329 & $1517-8382$ & 0.213 & 4.5 \\
\hline Brazilian Journal of Microbiology & v. 36 , fisrt page 114 , last page 117 & $1517-8382$ & 0.213 & 4.5 \\
\hline Brazilian Journal of Microbiology & v. 36 , fisrt page 246 , last page 252 & $1517-8382$ & 0.213 & 4.5 \\
\hline Brazilian Journal of Microbiology & v. 36 , fisrt page 315 , last page 320 & $1517-8382$ & 0.213 & 4.5 \\
\hline Brazilian Journal of Microbiology & v. 36 , fisrt page 327 , last page 332 & $1517-8382$ & 0.213 & 4.5 \\
\hline Brazilian Journal of Microbiology & v. 36 , fisrt page 333 , last page 337 & $1517-8382$ & 0.213 & 4.5 \\
\hline Brazilian Journal of Microbiology & v. 36 , fisrt page 35 , last page 37 & $1517-8382$ & 0.213 & 4.5 \\
\hline Brazilian Journal of Microbiology & v. 37 , fisrt page 148 , last page 152 & $1517-8382$ & 0.213 & 4.5 \\
\hline Brazilian Journal of Microbiology & v. 37 , fisrt page 244 , last page 252 & $1517-8382$ & 0.213 & 4.5 \\
\hline Brazilian Journal of Microbiology & v. 37 , fisrt page 290 , last page 295 & $1517-8382$ & 0.213 & 4.5 \\
\hline Brazilian Journal of Microbiology & v. 37 , fisrt page 401 , last page 416 & $1517-8382$ & 0.213 & 4.5 \\
\hline Brazilian Journal of Microbiology & v. 37 , fisrt page 425 , last page 427 & $1517-8382$ & 0.213 & 4.5 \\
\hline Brazilian Journal of Microbiology & v. 37 , fisrt page 468 , last page 473 & $1517-8382$ & 0.213 & 4.5 \\
\hline Brazilian Journal of Microbiology & v. 37 , fisrt page 495 , last page 499 & $1517-8382$ & 0.213 & 4.5 \\
\hline Brazilian Journal of Microbiology & v. 37, fisrt page 50 , last page 56 & $1517-8382$ & 0.213 & 4.5 \\
\hline Brazilian Journal of Microbiology & v. 64 , fisrt page 465 , last page 478 & $1517-8382$ & 0.213 & 4.5 \\
\hline Brittonia (Bronx) & v. 56 , fisrt page 147 , last page 150 & $0007-196 X$ & 0.211 & 10 \\
\hline Brittonia (Bronx) & v. 56 , fisrt page 288 , last page 290 & $0007-196 X$ & 0.211 & 10 \\
\hline Brittonia (Bronx) & v. 56 , fistt page 314 , last page 345 & $0007-196 X$ & 0.211 & 10 \\
\hline Brittonia (Bronx) & v. 56 , fistt page 353 , last page 356 & $0007-196 X$ & 0.211 & 10 \\
\hline Brittonia (Bronx) & v. 56, fisrt page 72, last page 74 & $0007-196 X$ & 0.211 & 10 \\
\hline Brittonia (Bronx) & v. 56 , fisrt page 75 , last page 81 & $0007-196 X$ & 0.211 & 10 \\
\hline Brittonia (Bronx) & v. 56, fisrt page 82, last page 88 & $0007-196 X$ & 0.211 & 10 \\
\hline Brittonia (Bronx) & v. 57 , fisrt page 123 , last page 128 & $0007-196 X$ & 0.211 & 10 \\
\hline Brittonia (Bronx) & v. 57 , fisrt page 248 , last page 251 & $0007-196 X$ & 0.211 & 10 \\
\hline Brittonia (Bronx) & v. 57 , fisrt page 28 , last page 34 & $0007-196 X$ & 0.211 & 10 \\
\hline Brittonia (Bronx) & v. 58 , fisrt page 10 , last page 41 & 0007-196X & 0.211 & 10 \\
\hline Brittonia (Bronx) & v. 58 , fisrt page 170 , last page 177 & $0007-196 X$ & 0.211 & 10 \\
\hline
\end{tabular}


Brittonia (Bronx)

Journal

Brittonia (Bronx)

Brittonia (Bronx)

Brittonia (Bronx)

New Zealand Journal of Crop and

Horticultural Science

Novon (Saint Louis)

Novon (Saint Louis)

Novon (Saint Louis)

Novon (Saint Louis)

Novon (Saint Louis)

Novon (Saint Louis)

Novon (Saint Louis)

Novon (Saint Louis)

Novon (Saint Louis)

Novon (Saint Louis)

Novon (Saint Louis)

Novon (Saint Louis)

Novon (Saint Louis)

Novon (Saint Louis)

Novon (Saint Louis)

Novon (Saint Louis)

Novon (Saint Louis)

Novon (Saint Louis)

Novon (Saint Louis)

Novon (Saint Louis)

Novon (Saint Louis)

Novon (Saint Louis)

Novon (Saint Louis)

Novon (Saint Louis)

Novon (Saint Louis)

Novon (Saint Louis)

Amazoniana (Kiel)

Amazoniana (Kiel)

Amazoniana (Kiel)

Amazoniana (Kiel)

Amazoniana (Kiel)

Amazoniana (Kiel)

Amazoniana (Kiel)

Amazoniana (Kiel)

Amazoniana (Kiel)

Agrociencia (Montecillo)

Agrociencia (Montecillo)

Agrociencia (Montecillo)

Arquivo Brasileiro de Medicina Veterinaria e Zootecnia

Arquivo Brasileiro de Medicina Veterinária e Zootecnia

Arquivo Brasileiro de Medicina Veterinária e Zootecnia

Arquivo Brasileiro de Medicina Veterinária e Zootecnia

Arquivo Brasileiro de Medicina Veterinária e Zootecnia

Arquivo Brasileiro de Medicina Veterinária

e Zootecnia

American Bee Journal

American Bee Journal

American Bee Journal

Acta Botanica Brasilica

Acta Botanica Brasilica

Acta Botanica Brasilica

Acta Botanica Brasilica
Paper info.

ISSN

v. 58, fisrt page 379 , last page 384

v. 58 , fisrt page 52 , last page 58

v. 60 , fisrt page 77 , last page 85

v. 9, fisrt page 160, last page 172

v. 34 , fisrt page 131, last page 137

v. 14, fisrt page 158, last page 162

v. 14 , fisrt page 168 , last page 170

v. 14, fisrt page 206, last page 209

v. 14 , fisrt page 233 , last page 235

v. 14 , fisrt page 236 , last page 238

v. 14 , fist page 29 , last page 32

v. 14 , fisrt page 315 , last page 321

v. 14 , fist page 33 , last page 35

v. 14, fisrt page 36 , last page 39

v. 14 , fisrt page 6 , last page 11

v. 15 , fisrt page 117 , last page 122

v. 15 , fist page 139 , last page 144

v. 15 , fisrt page 250 , last page 252

v. 15 , fisrt page 282 , last page 285

v. 15 , fist page 55 , last page 58

v. 15 , fisrt page 562 , last page 567

v. 15 , fisrt page 583 , last page 585

v. 15 , fisrt page 59 , last page 68

v. 16 , fist page 1 , last page 3

v. 16 , fisrt page 112 , last page 128

v. 16 , fisrt page 129, last page 132

v. 16 , fist page 155 , last page 157

v. 16 , fist page 17 , last page 22

v. 16 , fisrt page 212 , last page 215

v. 16, fisrt page 235, last page 239

v. 16 , fisrt page 421 , last page 423

v. 18 , fist page 1 , last page 10

v. 18 , fist page 173 , last page 184

v. 18 , fisrt page 241 , last page 249

v. 18 , fisrt page 251 , last page 266

v. 18 , fisrt page 273 , last page 288

v. 18 , fist page 29 , last page 47

v. 18 , fisrt page 299 , last page 315

v. 19 , fisrt page 1 , last page 32

v. XVIII, fisrt page 397, last page 404

v. 9, fisrt page 123, last page 132

v. 9 , fisrt page 61 , last page 66

v. 9 , fisrt page 67 , last page 71

v. 56, fisrt page 126, last page 129

v. 57 , fisrt page 562 , last page 564

v. 57 , fisrt page 565, last page 567

v. 58 , fist page 1229 , last page 1231

v. 58 , fisrt page 562 , last page 566

v. 58, fisrt page 969, last page 971

v. 144 , fisrt page 625 , last page 627

v. 144 , fisrt page 628 , last page 630

v. 145 , fisrt page 503, last page 505

v. 1 , fist page 26 , last page 36

v. 1 , fisrt page 653 , last page 657

v. 14 , fisrt page 763 , last page 768

v. 15 , fisrt page 167 , last page 181
0007-196X

0007-196X

0007-196X

0007-196X

0114-0671

1055-3177

1055-3177

1055-3177

1055-3177

1055-3177

1055-3177

1055-3177

1055-3177

1055-3177

1055-3177

1055-3177

1055-3177

1055-3177

1055-3177

1055-3177

1055-3177

1055-3177

1055-3177

1055-3177

1055-3177

1055-3177

1055-3177

1055-3177

1055-3177

1055-3177

1055-3177

0065-6755

0065-6755

0065-6755

0065-6755

0065-6755

0065-6755

0065-6755

0065-6755

0065-6755

1405-3195

1405-3195

1405-3195

0102-0935

0102-0935

0102-0935

0102-0935

0102-0935

0102-0935

0002-7626

0002-7626

0002-7626

0102-3306

0102-3306

0102-3306

0102-3306
Half-Life

$0.211 \quad 10$

$0.211 \quad 10$

$0.211 \quad 10$

$0.211 \quad 10$

$0.173 \quad 9.7$

$0.15 \quad 6.6$

$0.15 \quad 6.6$

$0.15 \quad 6.6$

$0.15 \quad 6.6$

$0.15 \quad 6.6$

$0.15 \quad 6.6$

$0.15 \quad 6.6$

$0.15 \quad 6.6$

$0.15 \quad 6.6$

$0.15 \quad 6.6$

$0.15 \quad 6.6$

$0.15 \quad 6.6$

$0.15 \quad 6.6$

$0.15 \quad 6.6$

$0.15 \quad 6.6$

$0.15 \quad 6.6$

$0.15 \quad 6.6$

$0.15 \quad 6.6$

$0.15 \quad 6.6$

$0.15 \quad 6.6$

$0.15 \quad 6.6$

$0.15 \quad 6.6$

$0.15 \quad 6.6$

$0.15 \quad 6.6$

$0.15 \quad 6.6$

$0.15 \quad 6.6$

$0.148 \quad 10$

$0.148 \quad 10$

$0.148 \quad 10$

$0.148 \quad 10$

$0.148 \quad 10$

$0.148 \quad 10$

$0.148 \quad 10$

$0.148 \quad 10$

$0.148 \quad 10$

0.123

0.123

0.123

$0.117 \quad 6$

$0.117 \quad 6$

$0.117 \quad 6$

$0.117 \quad 6$

$0.117 \quad 6$

$0.117 \quad 6$

$0.087 \quad 10$

$0.087 \quad 10$

$0.087 \quad 10$

0

0

0

0 
Journal

Paper info.

ISSN

Acta Botanica Brasilica

v. 18, fistt page 1, last page 201

0102-3306

Acta Botanica Brasilica

v. 18 , fisrt page 161 , last page 176

0102-3306

Acta Botanica Brasilica

v. 18 , fist page 19 , last page 29

0102-3306

Acta Botanica Brasilica

v. 18 , fisrt page 219 , last page 224

0102-3306

v. 18 , fisrt page 222 , last page 226

0102-3306

Acta Botanica Brasilica

v. 18 , fist page 241 , last page 251

$0102-3306$

v. 18 , fist page 253 , last page 260

0102-3306

Acta Botanica Brasilica

v. 18 , fisrt page 261 , last page 270

0102-3306

Acta Botanica Brasilica

v. 18 , fist page 271 , last page 280

0102-3306

v. 18 , fisrt page 281 , last page 293

0102-3306

v. 18 , fisrt page 284 , last page 293

v. 18 , fist page 295 , last page 304

v. 18 , fisrt page 305 , last page 318

v. 18 , fisrt page 31 , last page 35

v. 18 , fisrt page 319 , last page 331

v. 18 , fisrt page 341 , last page 348

v. 18 , fist page 351 , last page 358

v. 18 , fisrt page 359 , last page 367

v. 18 , fisrt page 369 , last page 374

v. 18 , fisrt page 375 , last page 380

v. 18 , fisrt page 381 , last page 390

v. 18, fisrt page 391, last page 399

v. 18 , fisrt page 401 , last page 406

v. 18 , fisrt page 407 , last page 423

v. 18 , fisrt page 431 , last page 435

v. 18 , fisrt page 437 , last page 458

v. 18 , fisrt page 49 , last page 71

v. 18 , fisrt page 503 , last page 512

v. 18 , fisrt page 537 , last page 553

v. 18 , fisrt page 573 , last page 580

v. 18 , fisrt page 581 , last page 589

v. 18 , fisrt page 591 , last page 600

v. 18 , fisrt page 601 , last page 612

v. 18 , fisrt page 643 , last page 652

v. 18, fisrt page 659 , last page 669

v. 18 , fisrt page 677 , last page 687

0102-3306

0102-3306

0102-3306

$0102-3306$

0102-3306

0102-3306

0102-3306

0102-3306

0102-3306

0102-3306

0102-3306

0102-3306

0102-3306

0102-3306

0102-3306

0102-3306

0102-3306

0102-3306

0102-3306

0102-3306

0102-3306

0102-3306

0102-3306

0102-3306

0102-3306

0102-3306

0102-3306

v. 18, fisrt page 689, last page 692

v. 18 , fist page 693 , last page 699

v. 18 , fisrt page 708 , last page 710

0102-3306

0102-3306

v. 18 , fisrt page 711 , last page 721

v. 18 , fist page 723 , last page 730

v. 18 , fist page 73 , last page 90

v. 18 , fisrt page 731 , last page 736

v. 18 , fisrt page 761 , last page 771

v. 18 , fist page 781 , last page 792

v. 18 , fist page 801 , last page 807

v. 18 , fisrt page 815 , last page 824

v. 18 , fisrt page 825 , last page 837

v. 18 , fisrt page 839 , last page 846

v. 18 , fisrt page 847 , last page 851

v. 18 , fisrt page 879 , last page 894

v. 18, fisrt page 903, last page 909

0102-3306

0102-3306

0102-3306

0102-3306

0102-3306

0102-3306

0102-3306

0102-3306

0102-3306

0102-3306

0102-3306

0102-3306

0102-3306

0102-3306

0102-3306

0102-3306

0102-3306

0102-3306

0102-3306

0102-3306

0102-3306

0

0

0

0

Half-Life

0

0

0

0

0

0

0

0

0

0

0

0

0

0

0

0

0

0

0

0

0

0

0

0

0

0

0

0

0

0

0

0

0

0

0

0 
Journal

0102-3306

Acta Botanica Brasilica

Acta Botanica Brasilica

Acta Botanica Brasilica

Acta Botanica Brasilica

Acta Botanica Brasilica

Acta Botanica Brasilica

Acta Botanica Brasilica

Acta Botanica Brasilica

Acta Botanica Brasilica

Acta Botanica Brasilica

Acta Botanica Brasilica

Acta Botanica Brasilica

Acta Botanica Brasilica

Acta Botanica Brasilica

Acta Botanica Brasilica

Acta Botanica Brasilica

Acta Botanica Brasilica

Acta Botanica Brasilica

Acta Botanica Brasilica

Acta Botanica Brasilica

Acta Botanica Brasilica

Acta Botanica Brasilica

Acta Botanica Brasilica

Acta Botanica Brasilica

Acta Botanica Brasilica

Acta Botanica Brasilica

Acta Botanica Brasilica

Acta Botanica Brasilica

Acta Botanica Brasilica

Acta Botanica Brasilica

Acta Botanica Brasilica

Acta Botanica Brasilica

Acta Botanica Brasilica

Acta Botanica Brasilica

Acta Botanica Brasilica

Acta Botanica Brasilica

Acta Botanica Brasilica

Acta Botanica Brasilica

Acta Botanica Brasilica

Acta Botanica Brasilica

Acta Botanica Brasilica

Acta Botanica Brasilica

Acta Botanica Brasilica

Acta Botanica Brasilica

Acta Botanica Brasilica

Acta Botanica Brasilica

Acta Botanica Brasilica

Acta Botanica Brasilica

Acta Botanica Brasilica

Acta Botanica Brasilica

Acta Botanica Brasilica

Acta Botanica Brasilica

Acta Botanica Brasilica

v. 19 , fist page 265 , last page 271

v. 19 , fisrt page 283 , last page 286

v. 19 , fisrt page 305 , last page 320

v. 19 , fist page 313 , last page 321

v. 19 , fisrt page 339 , last page 344

v. 19, fisrt page 345 , last page 357

v. 19 , fisrt page 359 , last page 368

v. 19 , fisrt page 369 , last page 376

v. 19 , fist page 39 , last page 44

v. 19 , fist page 391 , last page 397

v. 19 , fisrt page 425 , last page 429

v. 19 , fisrt page 443 , last page 449

v. 19 , fisrt page 45 , last page 60

v. 19 , fisrt page 451 , last page 464

v. 19 , fisrt page 473 , last page 486

v. 19 , fisrt page 487 , last page 492

v. 19 , fist page 501 , last page 510

v. 19 , fisrt page 515 , last page 518

v. 19 , fisrt page 519 , last page 525

v. 19 , fisrt page 539 , last page 547

v. 19 , fisrt page 549 , last page 561

v. 19 , fisrt page 561 , last page 566

v. 19 , fisrt page 563 , last page 572

v. 19 , fisrt page 567 , last page 574

v. 19 , fisrt page 573 , last page 578

v. 19 , fisrt page 579 , last page 586

v. 19 , fisrt page 587 , last page 596

v. 19 , fisrt page 597, last page 608

v. 19 , fisrt page 609 , last page 614

v. 19 , fisrt page 61 , last page 70

v. 19 , fisrt page 615 , last page 623

v. 19 , fisrt page 625 , last page 632

v. 19 , fisrt page 627 , last page 633

v. 19 , fisrt page 633, last page 637

v. 19 , fisrt page 657 , last page 667

v. 19 , fisrt page 669 , last page 678

v. 19 , fisrt page 729 , last page 734

v. 19 , fist page 755 , last page 763

v. 19 , fist page 775 , last page 781

v. 19 , fisrt page 783 , last page 788

v. 19 , fisrt page 803 , last page 813

v. 19 , fist page 81 , last page 90

v. 19 , fist page 813 , last page 817

v. 19 , fisrt page 819 , last page 834

v. 19 , fisrt page 835 , last page 841

v. 19 , fisrt page 837 , last page 843

v. 19 , fisrt page 843 , last page 858

v. 19 , fisrt page 867 , last page 877

v. 19 , fisrt page 869 , last page 889

v. 19 , fisrt page 927 , last page 934

v. 19 , fisrt page 935 , last page 943

v. 19, fisrt page 945, last page 961

v. 19 , fisrt page 963 , last page 977
0102-3306

0102-3306

0102-3306

0102-3306

0102-3306

0102-3306

0102-3306

0102-3306

0102-3306

0102-3306

0102-3306

0102-3306

0102-3306

0102-3306

0102-3306

0102-3306

0102-3306

0102-3306

0102-3306

0102-3306

0102-3306

0102-3306

0102-3306

0102-3306

0102-3306

0102-3306

0102-3306

0102-3306

0102-3306

0102-3306

0102-3306

0102-3306

0102-3306

0102-3306

0102-3306

0102-3306

0102-3306

0102-3306

0102-3306

0102-3306

0102-3306

0102-3306

0102-3306

0102-3306

0102-3306

0102-3306

0102-3306

0102-3306

0102-3306

0102-3306

0102-3306

0102-3306

0102-3306 
Journal

Paper info.

ISSN

Acta Botanica Brasilica

v. 19 , fist page 979 , last page 988

v. 20(2), fisrt page 313 , last page 327

0102-3306

Acta Botanica Brasilica

v. 20(2), fisrt page 367 , last page 382

0102-3306

0

Acta Botanica Brasilica

v. 20 , fisrt page 1 , last page 11

0102-3306

0

Acta Botanica Brasilica

Acta Botanica Brasilica

v. 20 , fisrt page 1 , last page 20

0102-3306

0

v. 20 , fisrt page 1 , last page 3

0102-3306

0

Acta Botanica Brasilica

v. 20 , fisrt page 115 , last page 124

$0102-3306$

0

0102-3306

Acta Botanica Brasilica

Acta Botanica Brasilica

Acta Botanica Brasilica

Acta Botanica Brasilica

Acta Botanica Brasilica

Acta Botanica Brasilica

Acta Botanica Brasilica

Acta Botanica Brasilica

Acta Botanica Brasilica

Acta Botanica Brasilica

Acta Botanica Brasilica

Acta Botanica Brasilica

Acta Botanica Brasilica

Acta Botanica Brasilica

Acta Botanica Brasilica

Acta Botanica Brasilica

Acta Botanica Brasilica

Acta Botanica Brasilica

Acta Botanica Brasilica

Acta Botanica Brasilica

Acta Botanica Brasilica

Acta Botanica Brasilica

Acta Botanica Brasilica

Acta Botanica Brasilica

Acta Botanica Brasilica

Acta Botanica Brasilica

Acta Botanica Brasilica

Acta Botanica Brasilica

Acta Botanica Brasilica

Acta Botanica Brasilica

Acta Botanica Brasilica

Acta Botanica Brasilica

Acta Botanica Brasilica

Acta Botanica Brasilica

Acta Botanica Brasilica

Acta Botanica Brasilica

Acta Botanica Brasilica

Acta Botanica Brasilica

Acta Botanica Brasilica

Acta Botanica Brasilica

Acta Botanica Brasilica

Acta Botanica Brasilica

Acta Botanica Brasilica

Acta Botanica Brasilica

Acta Botanica Brasilica

Acta Botanica Brasilica

Acta Botanica Brasilica

Acta Botanica Brasilica

Acta Botanica Brasilica

Acta Botânica Brasilica

Acta Botânica Brasilica

Acta Botanica Brasilica - SBB

Acta Botanica Brasilica, Acta Botanica

v. 20 , fist page 143 , last page 156

v. 20 , fisrt page 157 , last page 172

v. 20 , fisrt page 185 , last page 194

v. 20 , fisrt page 195, last page 201

v. 20 , fist page 207 , last page 224

v. 20, fisrt page 225, last page 239

v. 20 , fisrt page 249 , last page 256

v. 20 , fisrt page 25 , last page 30

v. 20 , fisrt page 258 , last page 269

v. 20 , fisrt page 265 , last page 272

v. 20 , fisrt page 289 , last page 3294

v. 20 , fisrt page 299 , last page 311

v. 20 , fisrt page 329 , last page 338

v. 20 , fisrt page 347 , last page 363

v. 20 , fisrt page 433 , last page 441

v. 20 , fisrt page 443 , last page 448

v. 20 , fisrt page 455 , last page 470

v. 20 , fisrt page 471 , last page 482

v. 20 , fisrt page 483 , last page 486

v. 20 , fisrt page 487 , last page 500

v. 20 , fist page 49 , last page 59

v. 20 , fisrt page 523 , last page 528

v. 20 , fisrt page 563 , last page 568

v. 20 , fisrt page 591 , last page 598

v. 20 , fisrt page 625 , last page 632

v. 20 , fisrt page 641 , last page 647

v. 20 , fisrt page 649 , last page 655

v. 20 , fisrt page 657 , last page 669

v. 20 , fisrt page 671 , last page 685

v. 20 , fist page 687 , last page 700

v. 20 , fisrt page 71 , last page 86

v. 20 , fisrt page 715 , last page 722

v. 20 , fisrt page 719 , last page 725

v. 20 , fisrt page 741 , last page 754

v. 20 , fisrt page 751 , last page 762

v. 20 , fisrt page 763 , last page 769

v. 20 , fisrt page 789 , last page 802

v. 20 , fist page 831 , last page 837

v. 20 , fist page 839 , last page 888

v. 20 , fist page 87 , last page 103

v. 20 , fisrt page 875 , last page 885

v. 20 , fisrt page 899 , last page 909

v. 20 , fisrt page 911 , last page 926

v. 20 , fisrt page 927 , last page 936

v. 20 , fisrt page 955 , last page 973

v. 20 , fisrt page 975 , last page 993

v. 27 , fisrt page 655 , last page 665

v. 39 , fist page 3217 , last page 3226

v. 53 , fisrt page 369 , last page 373

v. 18 , fisrt page 305 , last page 318

v. 18 , fisrt page 723 , last page 730

v. 19 , fisrt page 399 , last page 406

v. 18 , fisrt page 521 , last page 535

0102-3306

0102-3306

0102-3306

0102-3306

0102-3306

0102-3306

0102-3306

0102-3306

$0102-3306$

0102-3306

0102-3306

0102-3306

Half-Life

0102-3306

0102-3306

0102-3306

0102-3306

0102-3306

0102-3306

0102-3306

0102-3306

0102-3306

0102-3306

0102-3306

0102-3306

0102-3306

0102-3306

0102-3306

0102-3306

0102-3306

0102-3306

0102-3306

0102-3306

0102-3306

0102-3306

0102-3306

0102-3306

0102-3306

0102-3306

0102-3306

0102-3306

0102-3306

0102-3306

0102-3306

0102-3306

0102-3306

0102-3306

0102-3306

0102-3306

0102-3306

0102-3306

0102-3306

0102-3306

0102-3306

0

0

0

0

0

0

0

0

0

0

0

0

0

0

0

0

0

0

0

0

0

0

0

0

0

0

0

0 
Brazilian Journal of Biology Brazilian Journal of Biology Brazilian Journal of Biology Brazilian Journal of Biology Brazilian Journal of Biology Brazilian Journal of Biology Brazilian Journal of Biology Brazilian Journal of Biology Brazilian Journal of Biology Brazilian Journal of Biology Brazilian Journal of Biology Brazilian Journal of Biology Brazilian Journal of Biology Brazilian Journal of Biology Brazilian Journal of Biology Brazilian Journal of Biology Brazilian Journal of Biology Brazilian Journal of Biology Brazilian Journal of Biology Brazilian Journal of Biology Brazilian Journal of Biology Brazilian Journal of Biology Brazilian Journal of Biology Brazilian Journal of Biology Brazilian Journal of Biology Brazilian Journal of Biology Brazilian Journal of Biology Brazilian Journal of Biology Brazilian Journal of Biology Brazilian Journal of Biology Brazilian Journal of Biology Brazilian Journal of Biology Brazilian Journal of Biology Brazilian Journal of Biology Brazilian Journal of Biology Brazilian Journal of Biology Brazilian Journal of Biology Brazilian Journal of Biology Brazilian Journal of Biology Brazilian Journal of Biology Brazilian Journal of Biology Brazilian Journal of Biology Brazilian Journal of Biology Brazilian Journal of Biology Brazilian Journal of Biology Brazilian Journal of Biology Brazilian Journal of Biology Brazilian Journal of Biology Brazilian Journal of Biology Brazilian Journal of Biology Brazilian Journal of Biology Brazilian Journal of Biology Brazilian Journal of Biology Brazilian Journal of Biology Brazilian Journal of Biology Brazilian Journal of Biology Brazilian Journal of Biology Brazilian Journal of Biology Brazilian Journal of Biology v. 22 , fisrt page 523 , last page 536

1519-6984 0 v. 60 , fisrt page 75 , last page 83 v. 63 , fisrt page 47 , last page 60 v. 64 , fisrt page 1 , last page 15 v. 64 , fisrt page 1 , last page 4 v. 64 , fisrt page 1 , last page 8 v. 64 , fist page 11 , last page 26 v. 64 , fisrt page 165 , last page 168 v. 64, fisrt page 203, last page 209 v. 64, fisrt page 227, last page 235 v. 64 , fist page 25 , last page 31

v. 64 , fisrt page 309 , last page 316 v. 64 , fisrt page 327 , last page 336 v. 64 , fist page 33 , last page 39

v. 64 , fisrt page 357 , last page 362

v. 64 , fisrt page 371 , last page 382

v. 64 , fisrt page 383 , last page 398

v. 64 , fisrt page 399 , last page 406

v. 64 , fisrt page 423 , last page 445

v. 64 , fisrt page 459 , last page 463

v. 64 , fisrt page 523 , last page 529

v. 64 , fisrt page 531 , last page 541

v. 64 , fisrt page 543 , last page 549

v. 64 , fisrt page 553 , last page 555

v. 64 , fisrt page 563, last page 568

v. 64 , fisrt page 59 , last page 72

v. 64 , fisrt page 6 , last page 10

v. 64 , fisrt page 645 , last page 654

v. 64 , fisrt page 661 , last page 667

v. 64 , fisrt page 697 , last page 706

v. 64 , fisrt page 707 , last page 718

v. 64 , fisrt page 717 , last page 728

v. 64 , fisrt page 757 , last page 765

v. 64 , fisrt page 775 , last page 786

v. 64 , fisrt page 787 , last page 795

v. 64 , fisrt page 809 , last page 817

v. 64 , fisrt page 81 , last page 94

v. 64 , fisrt page 841 , last page 851

v. 64 , fisrt page 915 , last page 918

v. 65 , fisrt page 1 , last page 10

v. 65 , fisrt page 1 , last page 8

v. 65 , fisrt page 129, last page 139

v. 65 , fisrt page 141 , last page 157

v. 65 , fist page 169 , last page 177

v. 65 , fisrt page 187, last page 189

v. 65 , fisrt page 263 , last page 270

v. 65 , fisrt page 271 , last page 280

v. 65 , fist page 29 , last page 41

v. 65 , fisrt page 299 , last page 304

v. 65 , fisrt page 353 , last page 361

v. 65 , fisrt page 371 , last page 376

v. 65 , fisrt page 377 , last page 385

v. 65 , fisrt page 387 , last page 394

v. 65 , fisrt page 415 , last page 421

v. 65 , fisrt page 423 , last page 430

v. 65 , fisrt page 483 , last page 494

v. 65 , fisrt page 513 , last page 519

v. 65 , fisrt page 533 , last page 540

v. 65 , fisrt page 559 , last page 571
$1519-6984$

1519-6984

1519-6984

1519-6984

1519-6984

1519-6984

1519-6984

1519-6984

1519-6984

1519-6984

1519-6984

1519-6984

1519-6984

1519-6984

1519-6984

1519-6984

1519-6984

1519-6984

1519-6984

1519-6984

1519-6984

1519-6984

1519-6984

1519-6984

1519-6984

1519-6984

1519-6984

1519-6984

1519-6984

1519-6984

1519-6984

1519-6984

1519-6984

1519-6984

1519-6984

1519-6984

1519-6984

1519-6984

1519-6984

1519-6984

1519-6984

1519-6984

1519-6984

1519-6984

1519-6984

1519-6984

1519-6984

1519-6984

1519-6984

1519-6984

1519-6984

1519-6984

1519-6984

1519-6984

1519-6984

1519-6984
1519-6984

1519-6984
0

0

0

0

0

0

0

0

0

0

0

0

0

0

0

0

0

0

0

0

0

0

0

0

0

0

0

0

0

0

0

0

0

0

0

0

0

0

0

0

0

0

0

0

0

0

0 
Brazilian Journal of Biolog

Brazilian Journal of Biology

Brazilian Journal of Biology

Brazilian Journal of Biology

Brazilian Journal of Biology

Brazilian Journal of Biology

Brazilian Journal of Biology

Brazilian Journal of Biology

Brazilian Journal of Biology

Brazilian Journal of Biology

Brazilian Journal of Biology

Brazilian Journal of Biology

Brazilian Journal of Biology

Brazilian Journal of Biology

Brazilian Journal of Biology

Brazilian Journal of Biology

Brazilian Journal of Biology

Brazilian Journal of Biology

Brazilian Journal of Biology

Brazilian Journal of Biology

Brazilian Journal of Biology

Brazilian Journal of Biology

Brazilian Journal of Biology

Brazilian Journal of Biology

Brazilian Journal of Biology

Brazilian Journal of Biology

Brazilian Journal of Biology

Brazilian Journal of Biology

Brazilian Journal of Biology

Brazilian Journal of Biology

Brazilian Journal of Biology

Brazilian Journal of Biology

Brazilian Journal of Biology

Brazilian Journal of Biology

Brazilian Journal of Biology

Brazilian Journal of Biology

Brazilian Journal of Biology

Brazilian Journal of Biology

Brazilian Journal of Biology

Brazilian Journal of Biology

Brazilian Journal of Biology

Brazilian Journal of Biology

Brazilian Journal of Biology

Brazilian Journal of Biology

Brazilian Journal of Biology

Brazilian Journal of Biology

Brazilian Journal of Biology

Brazilian Journal of Biology

Brazilian Journal of Biology

Brazilian Journal of Biology

Brazilian Journal of Biology

Brazilian Journal of Plant Physiology

Brazilian Journal of Plant Physiology

Brazilian Journal of Plant Physiology

Brazilian Journal of Plant Physiology

Brazilian Journal of Plant Physiology

Brazilian Journal of Plant Physiology

Brazilian Journal of Plant Physiology

Brazilian Journal of Plant Physiology

Brazilian Journal of Plant Physiology
Paper info.

v. 65 , fisrt page 573 , last page 581

v. 65 , fisrt page 589 , last page 596

v. 65 , fisrt page 631 , last page 637

v. 65 , fisrt page 639 , last page 642

v. 65 , fisrt page 661 , last page 673

v. 65 , fisrt page 675 , last page 681

v. 65 , fisrt page 717 , last page 728

v. 66 , fisrt page 1 , last page 10

v. 66 , fisrt page 1 , last page 8

v. 66, fisrt page 101, last page 107

v. 66 , fist page 1051, last page 1055

v. 66 , fist page 1065, last page 1078

v. 66, fisrt page 1117, last page 1122

v. 66 , fisrt page 1137, last page 1139

v. 66 , fisrt page 185 , last page 187

v. 66 , fist page 19 , last page 24

v. 66 , fisrt page 233 , last page 238

v. 66 , fisrt page 239 , last page 261

v. 66 , fisrt page 263 , last page 277

v. 66, fisrt page 279, last page 293

v. 66 , fisrt page 29 , last page 41

v. 66 , fisrt page 301 , last page 308

v. 66 , fisrt page 35 , last page 44

v. 66, fisrt page 351 , last page 355

v. 66 , fisrt page 413 , last page 420

v. 66 , fisrt page 421 , last page 430

v. 66 , fisrt page 431 , last page 439

v. 66 , fisrt page 441 , last page 443

v. 66 , fisrt page 447 , last page 453

v. 66 , fisrt page 45 , last page 49

v. 66 , fisrt page 455 , last page 462

v. 66 , fisrt page 473 , last page 478

v. 66 , fisrt page 479 , last page 492

v. 66 , fisrt page 509 , last page 512

v. 66 , fisrt page 513 , last page 522

v. 66 , fisrt page 553 , last page 557

v. 66 , fisrt page 559 , last page 564

v. 66 , fisrt page 603 , last page 610

v. 66 , fisrt page 611 , last page 622

v. 66 , fisrt page 623 , last page 632

v. 66, fisrt page 651 , last page 659

v. 66 , fisrt page 681 , last page 696

v. 66 , fisrt page 747 , last page 754

v. 66 , fisrt page 765 , last page 779

v. 66, fisrt page 791, last page 802

v. 66 , fisrt page 853 , last page 862

v. 66 , fisrt page 9 , last page 17

v. 66 , fisrt page 945 , last page 954

v. 66 , fisrt page 955 , last page 957

v. 66 , fist page 999 , last page 1020

v. 67 , fisrt page 1 , last page 10

v. 16 , fisrt page 119 , last page 130

v. 16 , fisrt page 131 , last page 136

v. 16 , fisrt page 155 , last page 161

v. 16 , fist page 17 , last page 24

v. 16 , fisrt page 181 , last page 184

v. 16 , fisrt page 200 , last page 210

v. 16 , fist page 39 , last page 46

v. 16 , fisrt page 7 , last page 16

v. 16 , fist page 77 , last page 82
ISSN

1519-6984

1519-6984

$1519-6984$

1519-6984

1519-6984

1519-6984

1519-6984

1519-6984

1519-6984

1519-6984

$1519-6984$

1519-6984

$1519-6984$

1519-6984

0034-7108

1519-6984

$1519-6984$

1519-6984

1519-6984

1519-6984

1519-6984

1519-6984

1519-6984

1519-6984

1519-6984

1519-6984

1519-6984

1519-6984

1519-6984

0034-7108

1519-6984

1519-6984

1519-6984

1519-6984

1519-6984

1519-6984

1519-6984

1519-6984

1519-6984

1519-6984

1519-6984

1519-6984

1519-6984

1519-6984

1519-6984

1519-6984

1519-6984

1519-6984

0034-7108

1519-6984

1519-6984

1677-0420

1677-0420

1677-0420

1677-0420

1677-0420

1677-0420

1677-0420

1677-0420

1677-0420
Half-Life

0

0

0

0

0

0

0

0

0

0

0

0

0

0

0

0

0

0

0

0

0

0

0

0

0

0

0

0

0

0

0

0

0

0

0

0

0

0

0

0 
Journal Brazilian Journal of Plant Physiology Brazilian Journal of Plant Physiology Brazilian Journal of Plant Physiology Brazilian Journal of Plant Physiology Brazilian Journal of Plant Physiology Brazilian Journal of Plant Physiology Brazilian Journal of Plant Physiology Brazilian Journal of Plant Physiology Brazilian Journal of Plant Physiology Brazilian Journal of Plant Physiology Brazilian Journal of Plant Physiology Brazilian Journal of Plant Physiology Brazilian Journal of Plant Physiology Brazilian Journal of Plant Physiology Brazilian Journal of Plant Physiology Brazilian Journal of Plant Physiology Brazilian Journal of Plant Physiology Brazilian Journal of Plant Physiology Genetics and Molecular Research Genetics and Molecular Research Genetics and Molecular Research Genetics and Molecular Research Genetics and Molecular Research Genetics and Molecular Research Genetics and Molecular Research Genetics and Molecular Research Genetics and Molecular Research Genetics and Molecular Research Genetics and Molecular Research Genetics and Molecular Research Genetics and Molecular Research Genetics and Molecular Research Genetics and Molecular Research Genetics and Molecular Research Genetics and Molecular Research Iheringia. Série Zoologia Iheringia. Série Zoologia Iheringia. Série Zoologia Iheringia. Série Zoologia Iheringia. Série Zoologia Iheringia. Série Zoologia Iheringia. Série Zoologia Iheringia. Série Zoologia Iheringia. Série Zoologia Iheringia. Série Zoologia Iheringia. Série Zoologia Iheringia. Série Zoologia Iheringia. Série Zoologia Iheringia. Série Zoologia Iheringia. Série Zoologia Iheringia. Série Zoologia Iheringia. Série Zoologia Iheringia. Série Zoologia Iheringia. Série Zoologia Iheringia. Série Zoologia Iheringia. Série Zoologia Iheringia. Série Zoologia Iheringia. Série Zoologia Iheringia. Série Zoologia v. 17, fist page 1, last page 18

v. 17 , fisrt page 1 , last page 4

v. 17 , fisrt page 187 , last page 190

v. 17 , fisrt page 213 , last page 222

v. 17 , fisrt page 229 , last page 238

v. 17 , fisrt page 273 , last page 281

v. 17, fisrt page 291, last page 297

v. 17 , fisrt page 299, last page 306

v. 17 , fisrt page 307 , last page 313

v. 17 , fisrt page 325 , last page 334

v. 17 , fisrt page 335 , last page 343

v. 17 , fisrt page 401 , last page 410

v. 18 , fisrt page 269 , last page 280

v. 18 , fisrt page 299 , last page 305

v. 18 , fisrt page 315 , last page 323

v. 18 , fisrt page 325 , last page 331

v. 18 , fisrt page 333 , last page 339

v. 18 , fisrt page 359 , last page 365

v. 18 , fisrt page 95, last page 108

v. 05 , fisrt page 01 , last page 03

v. 05, fisrt page 91 , last page 92

v. 3, fisrt page 181, last page 194

v. 3, fisrt page 310 , last page 322

v. 3 , fisrt page 410 , last page 420

v. 4 , fisrt page 105 , last page 114

v. 4, fisrt page 177, last page 184

v. 4, fisrt page 185, last page 196

v. 4 , fisrt page 525 , last page 534

v. 4 , fist page 55 , last page 65

v. 4, fisrt page 684, last page 690

v. 4, fisrt page 704, last page 709

v. 4 , fisrt page 710 , last page 715

v. 4 , fisrt page 832 , last page 838

v. 5 , fisrt page 542 , last page 552

v. 5 , fisrt page 79 , last page 87

v. 6 , fisrt page 23 , last page 32

v. 19 , fisrt page 1 , last page 3

v. 26 , fisrt page 111 , last page 222

v. 7 , fisrt page 25 , last page 30

v. 92 , fisrt page 123, last page 132

v. 94 , fisrt page 117 , last page 122

v. 94 , fisrt page 133 , last page 135

v. 94 , fisrt page 135, last page 138

v. 94 , fisrt page 139 , last page 147

v. 94 , fisrt page 205 , last page 210

v. 94 , fisrt page 211 , last page 216

v. 94 , fisrt page 25 , last page 31

v. 94 , fisrt page 253, last page 259

v. 94 , fisrt page 261 , last page 268

v. 94 , fisrt page 271 , last page 276

v. 94 , fisrt page 285 , last page 294

v. 94, fisrt page 301 , last page 305

v. 94 , fisrt page 315 , last page 319

v. 94, fisrt page 329 , last page 333

v. 94 , fisrt page 357 , last page 373

v. 94 , fisrt page 375 , last page 380

v. 94, fisrt page 389, last page 393

v. 94 , fisrt page 421 , last page 424

v. 94 , fisrt page 433 , last page 438

v. 94 , fisrt page 443 , last page 446
ISSN

1677-0420

1677-0420

$1677-0420$

$1677-0420$

1677-0420

1677-0420

$1677-0420$

1677-0420

$1677-0420$

1677-0420

$1677-0420$

1677-0420

1677-0420

1677-0420

1677-0420

1677-0420

1677-0420

1677-0420

1677-0420

1676-5680

1676-5680

1676-5680

1676-5680

1676-5680

1676-5680

1676-5680

1676-5680

1676-5680

1676-5680

1676-5680

1676-5680

1676-5680

1676-5680

1676-5680

$1676-5680$

1676-5680

0073-4721

0073-4721

0073-4721

0073-4721

0073-4721

0073-4721

0073-4721

0073-4721

0073-4721

0073-4721

0073-4721

0073-4721

0073-4721

0073-4721

0073-4721

0073-4721

0073-4721

0073-4721

0073-4721

0073-4721

0073-4721

0073-4721

0073-4721

0073-4721 0 0

0

0

0

0

0

0

0

0

0

0

0

0

0

0

0

0

0

0

0

0

0

0

0

0

0

0

0

0

0

0

0

0

0

0

0

0

\section{0}

\section{0}

\section{0}

\section{0}

\section{0}

\section{0}

0

0

0

0 
Journa

Iheringia. Série Zoologia Iheringia. Série Zoologia Iheringia. Série Zoologia Iheringia. Série Zoologia Iheringia. Série Zoologia Iheringia. Série Zoologia Iheringia. Série Zoologia Iheringia. Série Zoologia Iheringia. Série Zoologia Iheringia. Série Zoologia Iheringia. Série Zoologia Iheringia. Série Zoologia Iheringia. Série Zoologia Iheringia. Série Zoologia Iheringia. Série Zoologia Iheringia. Série Zoologia Iheringia. Série Zoologia Iheringia. Série Zoologia Iheringia. Série Zoologia Iheringia. Série Zoologia Iheringia. Série Zoologia Iheringia. Série Zoologia Iheringia. Série Zoologia Iheringia. Série Zoologia Iheringia. Série Zoologia Iheringia. Série Zoologia Iheringia. Série Zoologia Iheringia. Série Zoologia Iheringia. Série Zoologia Iheringia. Série Zoologia Iheringia. Série Zoologia Iheringia. Série Zoologia Iheringia. Série Zoologia Iheringia. Série Zoologia Iheringia. Série Zoologia Iheringia. Série Zoologia Iheringia. Série Zoologia Iheringia. Série Zoologia Iheringia. Série Zoologia Iheringia. Série Zoologia Iheringia. Série Zoologia Iheringia. Série Zoologia Iheringia. Série Zoologia Iheringia. Série Zoologia Iheringia. Série Zoologia Iheringia. Série Zoologia Iheringia. Série Zoologia Iheringia. Série Zoologia Iheringia. Série Zoologia Iheringia. Série Zoologia Iheringia. Série Zoologia Iheringia. Série Zoologia Iheringia. Série Zoologia Iheringia. Série Zoologia Iheringia. Série Zoologia Iheringia. Série Zoologia Iheringia. Série Zoologia Iheringia. Série Zoologia Iheringia. Série Zoologia Iheringia. Série Zoologia v. 94 , fistt page 45 , last page 48

v. 94 , fisrt page 53 , last page 56

v. 94 , fisrt page 63 , last page 66

v. 94 , fisrt page 67 , last page 72

v. 94 , fisrt page 73 , last page 76

v. 94 , fist page 83 , last page 88

v. 94 , fist page 89 , last page 94

v. 94 , fisrt page 95 , last page 104

v. 95, fisrt page 103, last page 105

v. 95, fisrt page 121, last page 126

v. 95 , fisrt page 127 , last page 131

v. 95 , fisrt page 151 , last page 164

v. 95, fisrt page 165, last page 172

v. 95 , fisrt page 173 , last page 181

v. 95 , fisrt page 183 , last page 187

v. 95, fisrt page 189, last page 195

v. 95 , fisrt page 197, last page 203

v. 95, fisrt page 205, last page 212

v. 95 , fist page 21 , last page 24

v. 95, fisrt page 217 , last page 222

v. 95 , fisrt page 233 , last page 240

v. 95 , fisrt page 241 , last page 246

v. 95 , fisrt page 247 , last page 254

v. 95 , fisrt page 255 , last page 259

v. 95 , fist page 281 , last page 2954

v. 95 , fisrt page 29 , last page 46

v. 95 , fisrt page 295, last page 303

v. 95, fisrt page 305 , last page 310

v. 95, fisrt page 319 , last page 325

v. 95 , fisrt page 359 , last page 363

v. 95 , fisrt page 415 , last page 420

v. 95 , fisrt page 421 , last page 429

v. 95 , fisrt page 57 , last page 64

v. 95 , fist page 65 , last page 78

v. 95 , fist page 79 , last page 81

v. 95 , fist page 83 , last page 87

v. 95 , fisrt page 9 , last page 16

v. 95 , fisrt page 93 , last page 101

v. 96 , fisrt page 1 , last page 8

v. 96 , fisrt page 103, last page 108

v. 96 , fist page 115 , last page 119

v. 96 , fisrt page 135 , last page 140

v. 96 , fisrt page 147 , last page 163

v. 96 , fisrt page 165, last page 172

v. 96 , fisrt page 17 , last page 24

v. 96 , fisrt page 185, last page 196

v. 96, fisrt page 197, last page 204

v. 96, fisrt page 209, last page 212

v. 96 , fisrt page 237 , last page 248

v. 96 , fist page 25 , last page 30

v. 96 , fisrt page 257 , last page 260

v. 96 , fisrt page 273 , last page 384

v. 96 , fisrt page 289 , last page 291

v. 96 , fisrt page 299, last page 304

v. 96 , fisrt page 305 , last page 313

v. 96 , fist page 31 , last page 39

v. 96, fisrt page 315, last page 319

v. 96, fisrt page 321 , last page 327

v. 96, fisrt page 329 , last page 334

v. 96 , fisrt page 345 , last page 362
ISSN

0073-4721

$0073-4721$

0073-4721

0073-4721

0073-4721

0073-4721

0073-4721

0073-4721

0073-4721

0073-4721

0073-4721

0073-4721

0073-4721

0073-4721

$0073-4721$

0073-4721

0073-4721

0073-4721

$0073-4721$

0073-4721

0073-4721

0073-4721

0073-4721

0073-4721

0073-4721

0073-4721

0073-4721

0073-4721

0073-4721

0073-4721

0073-4721

0073-4721

0073-4721

0073-4721

0073-4721

0073-4721

0073-4721

0073-4721

0073-4721

0073-4721

0073-4721

0073-4721

0073-4721

0073-4721

0073-4721

0073-4721

0073-4721

0073-4721

0073-4721

0073-4721

0073-4721

0073-4721

0073-4721

0073-4721

0073-4721

0073-4721

0073-4721

0073-4721

0073-4721

0073-4721

Half-Life

0

0

0

0

0

0

0

0

0

0

0

0

0

0

0

0

0

$$
0
$$

0

\section{0}

\section{0}

0

0

\section{0}

\section{0}


Journa

Iheringia. Série Zoologia Iheringia. Série Zoologia Iheringia. Série Zoologia Iheringia. Série Zoologia Iheringia. Série Zoologia Iheringia. Série Zoologia Iheringia. Série Zoologia Iheringia. Série Zoologia Iheringia. Série Zoologia Iheringia. Série Zoologia Iheringia. Série Zoologia Iheringia. Série Zoologia Iheringia. Série Zoologia Iheringia. Série Zoologia Iheringia. Série Zoologia Iheringia. Série Zoologia Iheringia. Série Zoologia Iheringia. Série Zoologia Iheringia. Série Zoologia Iheringia. Série Zoologia Iheringia. Série Zoologia Iheringia. Série Zoologia Iheringia. Série Zoologia Kew Bulletin

Kew Bulletin

Kew Bulletin

Kew Bulletin

Kew Bulletin

Kew Bulletin

Kew Bulletin

Kew Bulletin

Kew Bulletin

Kew Bulletin

Kew Bulletin

Kew Bulletin

Kew Bulletin

Kew Bulletin

Kew Bulletin

Kew Bulletin

Kew Bulletin

Kew Bulletin

Kew Bulletin

Kew Bulletin

Kew Bulletin

Revista Arvore

Revista Árvore

Revista Árvore

Revista Árvore

Revista Árvore

Revista Árvore

Revista Árvore

Revista Árvore

Revista Árvore

Revista Árvore

Revista Árvore

Revista Árvore

Revista Árvore

Revista Árvore

Revista Árvore

Revista Árvore
Paper info.

v. 96 , fisrt page 373 , last page 375

v. 96 , fisrt page 377 , last page 381

v. 96 , fisrt page 387 , last page 390

v. 96 , fisrt page 41 , last page 45

v. 96 , fisrt page 413 , last page 417

v. 96 , fisrt page 419 , last page 424

v. 96 , fisrt page 431 , last page 435

v. 96 , fist page 437 , last page 444

v. 96 , fisrt page 445 , last page 451

v. 96 , fisrt page 453 , last page 465

v. 96 , fisrt page 467 , last page 472

v. 96 , fist page 47, last page 52

v. 96, fisrt page 473 , last page 477

v. 96 , fisrt page 479 , last page 485

v. 96 , fisrt page 5 , last page 12

v. 96 , fisrt page 53 , last page 56

v. 96 , fisrt page 57 , last page 60

v. 96 , fisrt page 61 , last page 64

v. 96 , fisrt page 67 , last page 70

v. 96 , fisrt page 75 , last page 80

v. 96 , fisrt page 81 , last page 87

v. 96 , fisrt page 89 , last page 93

v. 96 , fisrt page 95 , last page 101

v. 59, fisrt page 103, last page 109

v. 59 , fisrt page 149 , last page 151

v. 59 , fisrt page 261 , last page 267

v. 59 , fisrt page 285 , last page 290

v. 59 , fisrt page 297 , last page 300

v. 59, fisrt page 305, last page 309

v. 59 , fisrt page 4 , last page 7

v. 59 , fisrt page 497 , last page 520

v. 59 , fisrt page 531 , last page 539

v. 60 , fisrt page 103, last page 109

v. 60 , fisrt page 111 , last page 115

v. 60 , fisrt page 117 , last page 121

v. 60 , fisrt page 123 , last page 127

v. 60 , fisrt page 133 , last page 135

v. 60 , fisrt page 137 , last page 139

v. 60 , fisrt page 435 , last page 440

v. 60 , fisrt page 520 , last page 525

v. 60 , fist page 532 , last page 541

v. 60 , fisrt page 95 , last page 101

v. 61 , fisrt page 179 , last page 182

v. 61 , fisrt page 265 , last page 267

v. 28 , fist page 583 , last page 592

v. 28 , fisrt page 121 , last page 127

v. 28 , fisrt page 139 , last page 146

v. 28 , fist page 21 , last page 27

v. 28 , fisrt page 309 , last page 312

v. 28 , fisrt page 327 , last page 332

v. 28 , fisrt page 397 , last page 405

v. 28 , fisrt page 407 , last page 417

v. 28 , fisrt page 419 , last page 428

v. 28 , fisrt page 465 , last page 470

v. 28 , fisrt page 633 , last page 642

v. 28 , fisrt page 643 , last page 654

v. 28, fisrt page 681, last page 689

v. 28 , fisrt page 691 , last page 698

v. 28 , fisrt page 749 , last page 754

v. 28 , fisrt page 777 , last page 779
ISSN

0073-4721

$0073-4721$

Half-Life

0073-4721

0073-4721

0073-4721

0073-4721

0073-4721

0073-4721

0073-4721

0073-4721

0073-4721

0073-4721

0073-4721

0073-4721

0073-4721

0073-4721

0073-4721

0073-4721

0073-4721

0073-4721

0073-4721

0073-4721

0073-4721

0075-5974

0075-5974

0075-5974

0075-5974

0075-5974

0075-5974

0075-5974

0075-5974

0075-5974

0075-5974

0075-5974

0075-5974

0075-5974

0075-5974

0075-5974

0075-5974

0075-5974

0075-5974

0075-5974

0075-5974

0075-5974

0100-6762

0100-6762

0100-6762

0100-6762

0100-6762

0100-6762

0100-6762

0100-6762

0100-6762

0100-6762

0100-6762

0100-6762

0100-6762

0100-6762

0100-6762

0100-6762
0

0

0

0

0

\section{0}

0

0

0

0

0

0

0

0

0

0

0

0

\section{0}

0

\section{0}

0

0

0

0

0

0

0

0

0

0

0

\section{0}

\section{0}

\section{0}

\section{0}

\section{0}

\section{0}

0

0

0

0

0

0

0

0

0

0

0

0

0 


\begin{tabular}{|c|c|c|c|c|}
\hline Journal & Paper info. & ISSN & IF & Half-Life \\
\hline Revista Árvore & v. 29, fisrt page 139, last page 150 & $0100-6762$ & 0 & \\
\hline Revista Árvore & v. 29, fisrt page 25 , last page 34 & $0100-6762$ & 0 & \\
\hline Revista Árvore & v. 29 , fisrt page 35 , last page 46 & $0100-6762$ & 0 & \\
\hline Revista Árvore & v. 29 , fisrt page 517 , last page 524 & $0100-6762$ & 0 & \\
\hline Revista Árvore & v. 29 , fisrt page 553 , last page 561 & $0100-6762$ & 0 & \\
\hline Revista Árvore & v. 29, fisrt page 661, last page 669 & $0100-6762$ & 0 & \\
\hline Revista Árvore & v. 29, fisrt page 887 , last page 895 & $0100-6762$ & 0 & \\
\hline Revista Árvore & v. 29, fisrt page 907, last page 914 & $0100-6762$ & 0 & \\
\hline Revista Árvore & v. 29, fistt page 983 , last page 992 & $0100-6762$ & 0 & \\
\hline Revista Árvore & v. 29 , fisrt page 999, last page 1005 & $0100-6762$ & 0 & \\
\hline Revista Árvore & v. 30 , fisrt page 1025 , last page 1031 & $0100-6762$ & 0 & \\
\hline Revista Árvore & v. 30 , fisrt page 1045, last page 1054 & $0100-6762$ & 0 & \\
\hline Revista Árvore & v. 30 , fisrt page 351 , last page 357 & $0100-6762$ & 0 & \\
\hline Revista Árvore & v. 30, fisrt page 471, last page 479 & $0100-6762$ & 0 & \\
\hline Revista Árvore & v. 30 , fisrt page 481 , last page 489 & $0100-6762$ & 0 & \\
\hline Revista Árvore & v. 30 , fisrt page 49 , last page 54 & 0100-6762 & 0 & \\
\hline Revista Árvore & v. 30 , fisrt page 75 , last page 87 & $0100-6762$ & 0 & \\
\hline Revista Árvore & v. 30 , fisrt page 829 , last page 836 & $0100-6762$ & 0 & \\
\hline Revista Árvore & v. 30 , fisrt page 859 , last page 870 & $0100-6762$ & 0 & \\
\hline Revista Árvore & v. 30 , fisrt page 885 , last page 891 & $0100-6762$ & 0 & \\
\hline Revista Árvore & v. 30 , fisrt page 941 , last page 949 & $0100-6762$ & 0 & \\
\hline Revista Árvore & v. 30, fisrt page 99, last page 106 & $0100-6762$ & 0 & \\
\hline Revista Brasileira de Biologia & v. 65 , fisrt page 313 , last page 324 & 0034-7108 & 0 & \\
\hline Revista Brasileira de Biologia & v. 66 , fisrt page 709 , last page 717 & 0034-7108 & 0 & \\
\hline Revista Brasileira de Botânica & v. 18 , fisrt page 853 , last page 862 & $0100-8404$ & 0 & \\
\hline Revista Brasileira de Botânica & v. 25 , fisrt page 291 , last page 391 & $0100-8404$ & 0 & \\
\hline Revista Brasileira de Botânica & v. 27 , fisrt page 1 , last page 10 & $0100-8404$ & 0 & \\
\hline Revista Brasileira de Botânica & v. 27 , fisrt page 103 , last page 113 & $0100-8404$ & 0 & \\
\hline Revista Brasileira de Botânica & v. 27 , fisrt page 11 , last page 17 & $0100-8404$ & 0 & \\
\hline Revista Brasileira de Botânica & v. 27 , fisrt page 115 , last page 123 & $0100-8404$ & 0 & \\
\hline Revista Brasileira de Botânica & v. 27 , fisrt page 125 , last page 134 & $0100-8404$ & 0 & \\
\hline Revista Brasileira de Botânica & v. 27 , fisrt page 135 , last page 147 & $0100-8404$ & 0 & \\
\hline Revista Brasileira de Botânica & v. 27 , fisrt page 149 , last page 161 & 0100-8404 & 0 & \\
\hline Revista Brasileira de Botânica & v. 27 , fisrt page 163 , last page 183 & 0100-8404 & 0 & \\
\hline Revista Brasileira de Botânica & v. 27 , fisrt page 19 , last page 30 & $0100-8404$ & 0 & \\
\hline Revista Brasileira de Botânica & v. 27, fisrt page 193, last page 204 & $0100-8404$ & 0 & \\
\hline Revista Brasileira de Botânica & v. 27, fisrt page 213 , last page 227 & $0100-8404$ & 0 & \\
\hline Revista Brasileira de Botânica & v. 27 , fisrt page 241 , last page 248 & 0100-8404 & 0 & \\
\hline Revista Brasileira de Botânica & v. 27 , fisrt page 249 , last page 262 & $0100-8404$ & 0 & \\
\hline Revista Brasileira de Botânica & v. 27 , fisrt page 271 , last page 280 & $0100-8404$ & 0 & \\
\hline Revista Brasileira de Botânica & v. 27 , fisrt page 281 , last page 290 & 0100-8404 & 0 & \\
\hline Revista Brasileira de Botânica & v. 27 , fisrt page 311 , last page 323 & 0100-8404 & 0 & \\
\hline Revista Brasileira de Botânica & v. 27 , fisrt page 321 , last page 325 & $0100-8404$ & 0 & \\
\hline Revista Brasileira de Botânica & v. 27 , fisrt page 325 , last page 331 & 0100-8404 & 0 & \\
\hline Revista Brasileira de Botânica & v. 27 , fisrt page 333 , last page 335 & $0100-8404$ & 0 & \\
\hline Revista Brasileira de Botânica & v. 27 , fisrt page 337 , last page 348 & $0100-8404$ & 0 & \\
\hline Revista Brasileira de Botânica & v. 27 , fisrt page 363 , last page 377 & $0100-8404$ & 0 & \\
\hline Revista Brasileira de Botânica & v. 27 , fisrt page 37 , last page 45 & $0100-8404$ & 0 & \\
\hline Revista Brasileira de Botânica & v. 27 , fisrt page 379 , last page 393 & $0100-8404$ & 0 & \\
\hline Revista Brasileira de Botânica & v. 27 , fisrt page 395 , last page 406 & $0100-8404$ & 0 & \\
\hline Revista Brasileira de Botânica & v. 27 , fisrt page 407 , last page 419 & 0100-8404 & 0 & \\
\hline Revista Brasileira de Botânica & v. 27 , fisrt page 421 , last page 428 & $0100-8404$ & 0 & \\
\hline Revista Brasileira de Botânica & v. 27 , fisrt page 429 , last page 437 & $0100-8404$ & 0 & \\
\hline Revista Brasileira de Botânica & v. 27 , fisrt page 439 , last page 452 & 0100-8404 & 0 & \\
\hline Revista Brasileira de Botânica & v. 27 , fisrt page 453 , last page 462 & 0100-8404 & 0 & \\
\hline Revista Brasileira de Botânica & v. 27 , fisrt page 463 , last page 474 & 0100-8404 & 0 & \\
\hline Revista Brasileira de Botânica & v. 27 , fisrt page 47 , last page 56 & $0100-8404$ & 0 & \\
\hline Revista Brasileira de Botânica & v. 27 , fisrt page 475 , last page 487 & 0100-8404 & 0 & \\
\hline Revista Brasileira de Botânica & v. 27 , fisrt page 489 , last page 506 & 0100-8404 & 0 & \\
\hline Revista Brasileira de Botânica & v. 27 , fisrt page 507 , last page 513 & $0100-8404$ & 0 & \\
\hline
\end{tabular}


Journal

Revista Brasileira de Botânica

Revista Brasileira de Botânica

Revista Brasileira de Botânica

Revista Brasileira de Botânica

Revista Brasileira de Botânica

Revista Brasileira de Botânica

Revista Brasileira de Botânica

Revista Brasileira de Botânica

Revista Brasileira de Botânica

Revista Brasileira de Botânica

Revista Brasileira de Botânica

Revista Brasileira de Botânica

Revista Brasileira de Botânica

Revista Brasileira de Botânica

Revista Brasileira de Botânica

Revista Brasileira de Botânica

Revista Brasileira de Botânica

Revista Brasileira de Botânica

Revista Brasileira de Botânica

Revista Brasileira de Botânica

Revista Brasileira de Botânica

Revista Brasileira de Botânica

Revista Brasileira de Botânica

Revista Brasileira de Botânica

Revista Brasileira de Botânica

Revista Brasileira de Botânica

Revista Brasileira de Botânica

Revista Brasileira de Botânica

Revista Brasileira de Botânica

Revista Brasileira de Botânica

Revista Brasileira de Botânica

Revista Brasileira de Botânica

Revista Brasileira de Botânica

Revista Brasileira de Botânica

Revista Brasileira de Botânica

Revista Brasileira de Botânica

Revista Brasileira de Botânica

Revista Brasileira de Botânica

Revista Brasileira de Botânica

Revista Brasileira de Botânica

Revista Brasileira de Botânica

Revista Brasileira de Botânica

Revista Brasileira de Botânica

Revista Brasileira de Botânica

Revista Brasileira de Botânica

Revista Brasileira de Botânica

Revista Brasileira de Botânica

Revista Brasileira de Botânica

Revista Brasileira de Botânica

Revista Brasileira de Botânica

Revista Brasileira de Botânica

Revista Brasileira de Botânica

Revista Brasileira de Botânica

Revista Brasileira de Botânica

Revista Brasileira de Botânica

Revista Brasileira de Botânica

Revista Brasileira de Botânica

Revista Brasileira de Botânica

Revista Brasileira de Botânica
Paper info.

ISSN

0100-8404

v. 27, fisrt page 515, last page 525

v. 27 , fisrt page 527 , last page 532

v. 27 , fisrt page 545 , last page 561

0100-8404

0

v. 27 , fisrt page 55 , last page 61

v. 27 , fisrt page 563 , last page 572

v. 27 , fisrt page 587 , last page 596

v. 27 , fisrt page 607 , last page 613

v. 27 , fisrt page 629 , last page 638

v. 27, fisrt page 634, last page 639

v. 27 , fisrt page 639 , last page 641

v. 27 , fisrt page 641 , last page 653

v. 27 , fisrt page 655 , last page 665

v. 27 , fisrt page 667 , last page 670

v. 27 , fisrt page 71 , last page 78

v. 27 , fisrt page 713 , last page 723

v. 27 , fisrt page 725 , last page 737

v. 27 , fisrt page 739 , last page 755

v. 27 , fisrt page 767 , last page 779

v. 27 , fisrt page 769 , last page 777

v. 27 , fisrt page 78 , last page 84

v. 27 , fisrt page 781 , last page 785

v. 27 , fisrt page 787 , last page 796

v. 27 , fisrt page 79 , last page 84

v. 27 , fisrt page 797 , last page 804

v. 27, fisrt page 85, last page 102

v. 28 , fisrt page 1 , last page 10

v. 28 , fisrt page 1 , last page 22

v. 28 , fisrt page 1 , last page 3

v. 28 , fisrt page 109 , last page 135

v. 28 , fisrt page 137 , last page 147

v. 28 , fisrt page 149 , last page 161

v. 28 , fisrt page 163 , last page 170

v. 28, fisrt page 191, last page 202

v. 28 , fisrt page 219 , last page 228

v. 28 , fisrt page 229 , last page 240

v. 28 , fisrt page 23 , last page 37

v. 28 , fisrt page 255 , last page 265

v. 28 , fisrt page 267 , last page 275

v. 28 , fisrt page 277 , last page 283

v. 28 , fisrt page 285 , last page 294

v. 28 , fisrt page 305 , last page 318

v. 28 , fisrt page 319 , last page 328

v. 28 , fisrt page 361 , last page 373

v. 28 , fisrt page 375 , last page 387

v. 28 , fisrt page 389 , last page 397

v. 28 , fisrt page 419 , last page 421

v. 28 , fisrt page 423 , last page 439

v. 28 , fisrt page 441 , last page 450

v. 28 , fisrt page 451 , last page 466

v. 28 , fisrt page 467 , last page 476

0100-8404

0100-8404

0100-8404

0100-8404

0100-8404

0100-8404

0100-8404

0100-8404

0100-8404

0100-8404

0100-8404

0100-8404

0100-8404

0100-8404

0100-8404

0100-8404

0100-8404

0100-8404

0100-8404

0100-8404

0100-8404

0100-8404

0100-8404

0100-8404

0100-8404

0100-8404

0100-8404

0100-8404

0100-8404

0100-8404

0100-8404

0100-8404

0100-8404

0100-8404

0100-8404

0100-8404

0100-8404

0100-8404

0100-8404

0100-8404

0100-8404

0100-8404

0100-8404

0100-8404

0100-8404

0100-8404

0100-8404

0100-8404

0100-8404

0100-8404

0100-8404

0100-8404

0100-8404

0100-8404

0100-8404

0100-8404

0100-8404

0100-8404
0

0

0

0

0

0

0

0

0

0

0

\section{0}

0

0

0

0

0

0

0

0

0

0

0

0

0

0

0

\section{0}

0

0

0

0

0

0

0

0

0

0

0

0

0

0

\section{0}

\section{0}

\section{0}

0

0

0

0

0

0

0

0

0

0

0

0 
Journal

Revista Brasileira de Botânica

Revista Brasileira de Botânica

Revista Brasileira de Botânica

Revista Brasileira de Botânica

Revista Brasileira de Botânica

Revista Brasileira de Botânica

Revista Brasileira de Botânica

Revista Brasileira de Botânica

Revista Brasileira de Botânica

Revista Brasileira de Botânica

Revista Brasileira de Botânica

Revista Brasileira de Botânica

Revista Brasileira de Botânica

Revista Brasileira de Botânica

Revista Brasileira de Botânica

Revista Brasileira de Botânica

Revista Brasileira de Botânica

Revista Brasileira de Botânica

Revista Brasileira de Botânica

Revista Brasileira de Botânica

Revista Brasileira de Botânica

Revista Brasileira de Botânica

Revista Brasileira de Botânica

Revista Brasileira de Botânica

Revista Brasileira de Botânica

Revista Brasileira de Botânica

Revista Brasileira de Botânica

Revista Brasileira de Botânica

Revista Brasileira de Botânica

Revista Brasileira de Botânica

Revista Brasileira de Botânica

Revista Brasileira de Botânica

Revista Brasileira de Botânica

Revista Brasileira de Botânica

Revista Brasileira de Botânica

Revista Brasileira de Botânica

Revista Brasileira de Botânica

Revista Brasileira de Botânica

Revista Brasileira de Botânica

Revista Brasileira de Botânica

Revista Brasileira de Botânica

Revista Brasileira de Botânica

Revista Brasileira de Botânica

Revista Brasileira de Botânica

Revista Brasileira de Botânica

Revista Brasileira de Botânica

Revista Brasileira de Botânica

Revista Brasileira de Botânica

Revista Brasileira de Botânica

Revista Brasileira de Botânica

Revista Brasileira de Botânica

Revista Brasileira de Botânica

Revista Brasileira de Botânica

Revista Brasileira de Botânica

Revista Brasileira de Botânica

Revista Brasileira de Botânica

Revista Brasileira de Botânica

Revista Brasileira de Botânica

Revista Brasileira de Botânica
Paper info.

ISSN

0100-8404

v. 28 , fisrt page 687 , last page 696

v. 28 , fisrt page 697 , last page 712

v. 28 , fisrt page 735 , last page 744

0100-8404

0

0

v. 28 , fisrt page 75 , last page 84

v. 28 , fist page 755 , last page 763

v. 28 , fisrt page 765 , last page 772

v. 28 , fisrt page 773 , last page 783

v. 28 , fist page 835 , last page 839

v. 28 , fist page 95 , last page 108

v. 29 , fisrt page 1 , last page 3

v. 29 , fisrt page 115 , last page 131

v. 29 , fisrt page 13 , last page 25

v. 29 , fisrt page 131, last page 142

v. 29 , fisrt page 133, last page 144

v. 29 , fisrt page 145 , last page 162

v. 29 , fisrt page 161 , last page 172

v. 29 , fisrt page 163 , last page 174

v. 29 , fisrt page 173 , last page 180

v. 29 , fisrt page 183 , last page 186

v. 29 , fisrt page 185 , last page 190

v. 29, fisrt page 193, last page 207

v. 29, fisrt page 209, last page 215

v. 29 , fisrt page 217 , last page 228

v. 29 , fisrt page 229 , last page 238

v. 29 , fisrt page 239 , last page 249

v. 29, fisrt page 251, last page 258

v. 29 , fisrt page 267 , last page 273

v. 29 , fist page 27 , last page 37

v. 29 , fisrt page 275 , last page 287

v. 29 , fisrt page 289 , last page 299

v. 29, fisrt page 301 , last page 307

v. 29, fisrt page 309 , last page 318

v. 29 , fisrt page 319 , last page 330

v. 29 , fisrt page 331 , last page 333

v. 29 , fisrt page 335 , last page 337

v. 29, fisrt page 339 , last page 352

v. 29 , fisrt page 363 , last page 377

v. 29 , fisrt page 379 , last page 389

v. 29 , fist page 39 , last page 47

v. 29, fisrt page 391, last page 397

v. 29 , fisrt page 415 , last page 421

v. 29 , fisrt page 423 , last page 432

v. 29 , fisrt page 461 , last page 470

v. 29 , fisrt page 471 , last page 480

v. 29 , fisrt page 49 , last page 65

v. 29 , fisrt page 497, last page 508

v. 29 , fisrt page 509 , last page 530

v. 29 , fisrt page 555 , last page 567

v. 29 , fisrt page 569 , last page 577

v. 29 , fisrt page 579 , last page 586

v. 29, fisrt page 595, last page 602

v. 29 , fisrt page 603 , last page 607

v. 29 , fisrt page 639 , last page 656

v. 29 , fisrt page 657 , last page 667

v. 29 , fisrt page 669 , last page 680

v. 29 , fisrt page 67 , last page 77

v. 29 , fisrt page 681 , last page 687

v. 29 , fisrt page 709 , last page 720

v. 29, fist page 91 , last page 99

v. 3 , fisrt page 489 , last page 495
0100-8404

0100-8404

0

0100-8404

0100-8404

0100-8404

0100-8404

0100-8404

0100-8404

0100-8404

0100-8404

0100-8404

0100-8404

0100-8404

0100-8404

0100-8404

0100-8404

0100-8404

0100-8404

0100-8404

0100-8404

0100-8404

0100-8404

0100-8404

0100-8404

0100-8404

0100-8404

0100-8404

0100-8404

0100-8404

0100-8404

0100-8404

0100-8404

0100-8404

0100-8404

0100-8404

0100-8404

0100-8404

0100-8404

0100-8404

0100-8404

0100-8404

0100-8404

0100-8404

0100-8404

0100-8404

0100-8404

0100-8404

0100-8404

0100-8404

0100-8404

0100-8404

0100-8404

0100-8404

0100-8404

0100-8404

0100-8404

0100-8404

0100-8404

0

0

0

0

0

0

0

\section{0}

\section{0}

\section{0}

0

0

0

0

0

0

0

0

0

0

0

0

0

0

0

\section{0}

0

0

0

0

Half-Life 


\begin{tabular}{|c|c|c|c|c|}
\hline Journal & Paper info. & ISSN & IF & Half-Life \\
\hline Revista Brasileira de Botânica & v. 64 , fisrt page 669, last page 676 & $0100-8404$ & 0 & \\
\hline Revista Brasileira de Entomologia & v. 1 , fistt page 1 , last page 12 & $0085-5626$ & 0 & \\
\hline Revista Brasileira de Entomologia & v. 2, fisrt page 331 , last page 334 & $0085-5626$ & 0 & \\
\hline Revista Brasileira de Entomologia & v. 21 , fisrt page 833 , last page 846 & $0085-5626$ & 0 & \\
\hline Revista Brasileira de Entomologia & v. 3, fisrt page 339 , last page 341 & $0085-5626$ & 0 & \\
\hline Revista Brasileira de Entomologia & v. 3 , fisrt page 431 , last page 431 & $0085-5626$ & 0 & \\
\hline Revista Brasileira de Entomologia & v. 44, fisrt page 291, last page 293 & $0085-5626$ & 0 & \\
\hline Revista Brasileira de Entomologia & v. 48 , fisrt page 101, last page 104 & $0085-5626$ & 0 & \\
\hline Revista Brasileira de Entomologia & v. 48 , fisrt page 105 , last page 113 & $0085-5626$ & 0 & \\
\hline Revista Brasileira de Entomologia & v. 48 , fisrt page 115 , last page 118 & $0085-5626$ & 0 & \\
\hline Revista Brasileira de Entomologia & v. 48 , fisrt page 133, last page 135 & $0085-5626$ & 0 & \\
\hline Revista Brasileira de Entomologia & v. 48 , fisrt page 163, last page 167 & $0085-5626$ & 0 & \\
\hline Revista Brasileira de Entomologia & v. 48 , fisrt page 193, last page 197 & $0085-5626$ & 0 & \\
\hline Revista Brasileira de Entomologia & v. 48 , fisrt page 203, last page 220 & $0085-5626$ & 0 & \\
\hline Revista Brasileira de Entomologia & v. 48 , fisrt page 221 , last page 225 & $0085-5626$ & 0 & \\
\hline Revista Brasileira de Entomologia & v. 48 , fisrt page 227, last page 229 & $0085-5626$ & 0 & \\
\hline Revista Brasileira de Entomologia & v. 48 , fisrt page 233 , last page 238 & $0085-5626$ & 0 & \\
\hline Revista Brasileira de Entomologia & v. 48 , fisrt page 239 , last page 241 & $0085-5626$ & 0 & \\
\hline Revista Brasileira de Entomologia & v. 48 , fisrt page 273 , last page 282 & $0085-5626$ & 0 & \\
\hline Revista Brasileira de Entomologia & v. 48 , fisrt page 283 , last page 286 & $0085-5626$ & 0 & \\
\hline Revista Brasileira de Entomologia & v. 48 , fisrt page 287 , last page 289 & $0085-5626$ & 0 & \\
\hline Revista Brasileira de Entomologia & v. 48 , fisrt page 291 , last page 293 & $0085-5626$ & 0 & \\
\hline Revista Brasileira de Entomologia & v. 48 , fisrt page 31 , last page 34 & $0085-5626$ & 0 & \\
\hline Revista Brasileira de Entomologia & v. 48 , fisrt page 315 , last page 322 & $0085-5626$ & 0 & \\
\hline Revista Brasileira de Entomologia & v. 48 , fisrt page 323 , last page 325 & $0085-5626$ & 0 & \\
\hline Revista Brasileira de Entomologia & v. 48 , fisrt page 335 , last page 338 & $0085-5626$ & 0 & \\
\hline Revista Brasileira de Entomologia & v. 48 , fisrt page 343 , last page 346 & $0085-5626$ & 0 & \\
\hline Revista Brasileira de Entomologia & v. 48 , fisrt page 353 , last page 377 & $0085-5626$ & 0 & \\
\hline Revista Brasileira de Entomologia & v. 48 , fisrt page 379 , last page 382 & $0085-5626$ & 0 & \\
\hline Revista Brasileira de Entomologia & v. 48 , fisrt page 383 , last page 389 & $0085-5626$ & 0 & \\
\hline Revista Brasileira de Entomologia & v. 48 , fisrt page 39, last page 43 & $0085-5626$ & 0 & \\
\hline Revista Brasileira de Entomologia & v. 48 , fisrt page 395, last page 400 & $0085-5626$ & 0 & \\
\hline Revista Brasileira de Entomologia & v. 48, fisrt page 415 , last page 420 & $0085-5626$ & 0 & \\
\hline Revista Brasileira de Entomologia & v. 48 , fisrt page 421 , last page 430 & $0085-5626$ & 0 & \\
\hline Revista Brasileira de Entomologia & v. 48 , fisrt page 433 , last page 438 & $0085-5626$ & 0 & \\
\hline Revista Brasileira de Entomologia & v. 48 , fisrt page 459 , last page 466 & $0085-5626$ & 0 & \\
\hline Revista Brasileira de Entomologia & v. 48 , fisrt page 473 , last page 480 & $0085-5626$ & 0 & \\
\hline Revista Brasileira de Entomologia & v. 48 , fisrt page 481 , last page 484 & $0085-5626$ & 0 & \\
\hline Revista Brasileira de Entomologia & v. 48 , fisrt page 485 , last page 490 & $0085-5626$ & 0 & \\
\hline Revista brasileira de Entomologia & v. 48 , fisrt page 495, last page 508 & $0085-5626$ & 0 & \\
\hline Revista Brasileira de Entomologia & v. 48 , fisrt page 519 , last page 528 & $0085-5626$ & 0 & \\
\hline Revista Brasileira de Entomologia & v. 48 , fisrt page 535 , last page 545 & $0085-5626$ & 0 & \\
\hline Revista Brasileira de Entomologia & v. 48 , fisrt page 569 , last page 576 & $0085-5626$ & 0 & \\
\hline Revista Brasileira de Entomologia & v. 48, fisrt page 577, last page 579 & $0085-5626$ & 0 & \\
\hline Revista Brasileira de Entomologia & v. 48, fisrt page 581, last page 582 & $0085-5626$ & 0 & \\
\hline Revista Brasileira de Entomologia & v. 48 , fisrt page 59 , last page 63 & $0085-5626$ & 0 & \\
\hline Revista Brasileira de Entomologia & v. 48 , fisrt page 77 , last page 90 & $0085-5626$ & 0 & \\
\hline Revista Brasileira de Entomologia & v. 49, fisrt page 1 , last page 7 & $0085-5626$ & 0 & \\
\hline Revista Brasileira de Entomologia & v. 49, fisrt page 107, last page 117 & $0085-5626$ & 0 & \\
\hline Revista Brasileira de Entomologia & v. 49 , fisrt page 130 , last page 140 & $0085-5626$ & 0 & \\
\hline Revista Brasileira de Entomologia & v. 49 , fisrt page 148 , last page 151 & $0085-5626$ & 0 & \\
\hline Revista Brasileira de Entomologia & v. 49, fisrt page 15 , last page 28 & $0085-5626$ & 0 & \\
\hline Revista Brasileira de Entomologia & v. 49, fisrt page 167, last page 169 & $0085-5626$ & 0 & \\
\hline Revista Brasileira de Entomologia & v. 49 , fisrt page 171 , last page 180 & $0085-5626$ & 0 & \\
\hline Revista Brasileira de Entomologia & v. 49, fisrt page 181, last page 204 & $0085-5626$ & 0 & \\
\hline Revista Brasileira de Entomologia & v. 49, fisrt page 210 , last page 227 & $0085-5626$ & 0 & \\
\hline Revista Brasileira de Entomologia & v. 49 , fisrt page 228 , last page 236 & $0085-5626$ & 0 & \\
\hline Revista Brasileira de Entomologia & v. 49, fisrt page 240 , last page 244 & $0085-5626$ & 0 & \\
\hline Revista Brasileira de Entomologia & v. 49 , fisrt page 245 , last page 248 & $0085-5626$ & 0 & \\
\hline Revista Brasileira de Entomologia & v. 49, fisrt page 25 , last page 26 & $0085-5626$ & 0 & \\
\hline
\end{tabular}


Journal Revista Brasileira de Entomologia Revista Brasileira de Entomologia Revista Brasileira de Entomologia Revista Brasileira de Entomologia Revista Brasileira de Entomologia Revista Brasileira de Entomologia Revista Brasileira de Entomologia Revista Brasileira de Entomologia Revista Brasileira de Entomologia Revista Brasileira de Entomologia Revista Brasileira de Entomologia Revista Brasileira de Entomologia Revista Brasileira de Entomologia Revista Brasileira de Entomologia Revista Brasileira de Entomologia Revista Brasileira de Entomologia Revista Brasileira de Entomologia Revista Brasileira de Entomologia Revista Brasileira de Entomologia Revista Brasileira de Entomologia Revista Brasileira de Entomologia Revista Brasileira de Entomologia Revista Brasileira de Entomologia Revista Brasileira de Entomologia Revista Brasileira de Entomologia Revista Brasileira de Entomologia Revista Brasileira de Entomologia Revista Brasileira de Entomologia Revista Brasileira de Entomologia Revista Brasileira de Entomologia Revista Brasileira de Entomologia Revista Brasileira de Entomologia Revista Brasileira de Entomologia Revista Brasileira de Entomologia Revista Brasileira de Entomologia Revista Brasileira de Entomologia Revista Brasileira de Entomologia Revista Brasileira de Entomologia Revista Brasileira de Entomologia Revista Brasileira de Entomologia Revista Brasileira de Entomologia Revista Brasileira de Entomologia Revista Brasileira de Entomologia Revista Brasileira de Entomologia Revista Brasileira de Entomologia Revista Brasileira de Entomologia Revista Brasileira de Entomologia Revista Brasileira de Entomologia Revista Brasileira de Entomologia Revista Brasileira de Entomologia Revista Brasileira de Entomologia Revista Brasileira de Entomologia Revista Brasileira de Entomologia Revista Brasileira de Entomologia Revista Brasileira de Entomologia Revista Brasileira de Entomologia Revista Brasileira de Entomologia Revista Brasileira de Entomologia Revista Brasileira de Entomologia v. 49, fisrt page 253, last page 263

v. 49 , fisrt page 264 , last page 269

v. 49 , fisrt page 270 , last page 274

v. 49 , fisrt page 275 , last page 287

v. 49 , fisrt page 29 , last page 35

v. 49, fisrt page 294, last page 339

v. 49 , fisrt page 340 , last page 346

v. 49, fisrt page 347 , last page 352

v. 49 , fisrt page 353 , last page 366

v. 49 , fisrt page 367 , last page 368

v. 49 , fisrt page 392 , last page 396

v. 49 , fisrt page 397 , last page 408

v. 49 , fisrt page 415 , last page 420

v. 49 , fisrt page 421 , last page 424

v. 49 , fisrt page 427 , last page 429

v. 49 , fisrt page 446 , last page 447

v. 49 , fisrt page 448 , last page 452

v. 49, fisrt page 512, last page 521

v. 49 , fisrt page 527 , last page 530

v. 49 , fisrt page 557 , last page 571

v. 49 , fisrt page 572 , last page 574

v. 49 , fisrt page 580 , last page 583

v. 49 , fisrt page 69, last page 92

v. 49 , fisrt page 7 , last page 14

v. 49 , fist page 93 , last page 96

v. 49 , fisrt page 97 , last page 106

v. 50 (3), fisrt page 352, last page 354

v. 50(4), fisrt page 74 , last page 79

v. 50, fisrt page 101, last page 109

v. 50 , fisrt page 110 , last page 114

v. 50 , fisrt page 115 , last page 118

v. 50 , fisrt page 128 , last page 130

v. 50 , fisrt page 131 , last page 134

v. 50, fisrt page 137, last page 152

v. 50 , fisrt page 165 , last page 231

v. 50 , fisrt page 232, last page 239

v. 50, fisrt page 257, last page 267

v. 50 , fisrt page 268 , last page 274

v. 50 , fisrt page 275 , last page 279

v. 50 , fisrt page 280 , last page 286

v. 50, fisrt page 287, last page 292

v. 50, fisrt page 293, last page 296

v. 50 , fisrt page 297, last page 334

v. 50 , fist page 31 , last page 33

v. 50 , fisrt page 335 , last page 340

v. 50 , fisrt page 341 , last page 346

v. 50 , fisrt page 347 , last page 351

v. 50 , fisrt page 355 , last page 378

v. 50 , fisrt page 379 , last page 384

v. 50 , fist page 39 , last page 42

v. 50 , fisrt page 406 , last page 412

v. 50 , fisrt page 419 , last page 422

v. 50 , fisrt page 423 , last page 430

v. 50 , fisrt page 439 , last page 449

v. 50 , fisrt page 450 , last page 452

v. 50 , fisrt page 463 , last page 472

v. 50 , fisrt page 473 , last page 475

v. 50 , fisrt page 488 , last page 491

v. 50 , fisrt page 49 , last page 52

v. 50 , fisrt page 528 , last page 533
ISSN

0085-5626

0085-5626

0085-5626

0085-5626

0085-5626

0085-5626

0085-5626

0085-5626

0085-5626

0085-5626

0085-5626

0085-5626

0085-5626

0085-5626

0085-5626

0085-5626

0085-5626

0085-5626

0085-5626

0085-5626

0085-5626

0085-5626

0085-5626

0085-5626

0085-5626

0085-5626

0085-5626

0085-5626

0085-5626

0085-5626

0085-5626

0085-5626

0085-5626

0085-5626

0085-5626

0085-5626

0085-5626

0085-5626

0085-5626

0085-5626

0085-5626

0085-5626

0085-5626

0085-5626

0085-5626

0085-5626

0085-5626

0085-5626

0085-5626

0085-5626

0085-5626

0085-5626

0085-5626

0085-5626

0085-5626

0085-5626

0085-5626

0085-5626

0085-5626

0085-5626
IF 0 0

0

0

0

0

0

0

0

0

0

0

0

\section{0}

0

\section{0}

0

0

0

0

0

0

0

0

0

0

0

0

0

0

\section{0}




\begin{tabular}{|c|c|c|c|c|}
\hline Journal & Paper info. & ISSN & IF & Half-Life \\
\hline Revista Brasileira de Entomologia & v. 50 , fisrt page 53 , last page 63 & $0085-5626$ & 0 & \\
\hline Revista Brasileira de Entomologia & v. 50 , fisrt page 540 , last page 546 & $0085-5626$ & 0 & \\
\hline Revista Brasileira de Entomologia & v. 50 , fisrt page 6 , last page 30 & $0085-5626$ & 0 & \\
\hline Revista Brasileira de Entomologia & v. 50, fisrt page 76 , last page 79 & $0085-5626$ & 0 & \\
\hline Revista Brasileira de Entomologia & v. 50 , fisrt page 93 , last page 100 & $0085-5626$ & 0 & \\
\hline Revista Brasileira de Entomologia & v. 9, fisrt page 34 , last page 41 & $0085-5626$ & 0 & \\
\hline Revista Brasileira de Zoologia & v. 1, fisrt page 891 , last page 897 & 0101-8175 & 0 & \\
\hline Revista Brasileira de Zoologia & v. 1 , fisrt page 97 , last page 114 & $0101-8175$ & 0 & \\
\hline Revista Brasileira de Zoologia & v. 21 , fisrt page 1 , last page 8 & $0101-8175$ & 0 & \\
\hline Revista Brasileira de Zoologia & v. 21, fisrt page 1001 , last page 1011 & $0101-8175$ & 0 & \\
\hline Revista Brasileira de Zoologia & v. 21, fisrt page 1011, last page 1015 & 0101-8175 & 0 & \\
\hline Revista Brasileira de Zoologia & v. 21 , fisrt page 115 , last page 117 & 0101-8175 & 0 & \\
\hline Revista Brasileira de Zoologia & v. 21 , fisrt page 117 , last page 122 & $0101-8175$ & 0 & \\
\hline Revista Brasileira de Zoologia & v. 21 , fisrt page 123 , last page 126 & $0101-8175$ & 0 & \\
\hline Revista Brasileira de Zoologia & v. 21 , fisrt page 137, last page 144 & 0101-8175 & 0 & \\
\hline Revista Brasileira de Zoologia & v. 21 , fisrt page 15 , last page 25 & 0101-8175 & 0 & \\
\hline Revista Brasileira de Zoologia & v. 21 , fisrt page 153 , last page 155 & $0101-8175$ & 0 & \\
\hline Revista Brasileira de Zoologia & v. 21 , fisrt page 155 , last page 157 & $0101-8175$ & 0 & \\
\hline Revista Brasileira de Zoologia & v. 21 , fisrt page 157, last page 159 & $0101-8175$ & 0 & \\
\hline Revista Brasileira de Zoologia & v. 21 , fisrt page 161 , last page 168 & $0101-8175$ & 0 & \\
\hline Revista Brasileira de Zoologia & v. 21 , fisrt page 185, last page 187 & $0101-8175$ & 0 & \\
\hline Revista Brasileira de Zoologia & v. 21, fisrt page 189, last page 192 & 0101-8175 & 0 & \\
\hline Revista Brasileira de Zoologia & v. 21 , fisrt page 193 , last page 200 & $0101-8175$ & 0 & \\
\hline Revista Brasileira de Zoologia & v. 21 , fisrt page 207 , last page 218 & $0101-8175$ & 0 & \\
\hline Revista Brasileira de Zoologia & v. 21, fisrt page 219 , last page 227 & $0101-8175$ & 0 & \\
\hline Revista Brasileira de Zoologia & v. 21 , fisrt page 229 , last page 232 & 0101-8175 & 0 & \\
\hline Revista Brasileira de Zoologia & v. 21, fisrt page 243 , last page 247 & $0101-8175$ & 0 & \\
\hline Revista Brasileira de Zoologia & v. 21 , fisrt page 249, last page 252 & $0101-8175$ & 0 & \\
\hline Revista Brasileira de Zoologia & v. 21, fisrt page 253, last page 259 & $0101-8175$ & 0 & \\
\hline Revista Brasileira de Zoologia & v. 21 , fisrt page 261 , last page 266 & 0101-8175 & 0 & \\
\hline Revista Brasileira de Zoologia & v. 21 , fisrt page 267, last page 271 & 0101-8175 & 0 & \\
\hline Revista Brasileira de Zoologia & v. 21 , fisrt page 273 , last page 276 & $0101-8175$ & 0 & \\
\hline Revista Brasileira de Zoologia & v. 21 , fisrt page 277 , last page 280 & $0101-8175$ & 0 & \\
\hline Revista Brasileira de Zoologia & v. 21 , fisrt page 283 , last page 285 & 0101-8175 & 0 & \\
\hline Revista Brasileira de Zoologia & v. 21 , fisrt page 287, last page 293 & $0101-8175$ & 0 & \\
\hline Revista Brasileira de Zoologia & v. 21 , fisrt page 307 , last page 308 & $0101-8175$ & 0 & \\
\hline Revista Brasileira de Zoologia & v. 21, fisrt page 309 , last page 314 & 0101-8175 & 0 & \\
\hline Revista Brasileira de Zoologia & v. 21 , fisrt page 319 , last page 324 & 0101-8175 & 0 & \\
\hline Revista Brasileira de Zoologia & v. 21 , fisrt page 325 , last page 331 & $0101-8175$ & 0 & \\
\hline Revista Brasileira de Zoologia & v. 21 , fisrt page 337 , last page 342 & $0101-8175$ & 0 & \\
\hline Revista Brasileira de Zoologia & v. 21, fisrt page 343 , last page 344 & 0101-8175 & 0 & \\
\hline Revista Brasileira de Zoologia & v. 21 , fisrt page 345 , last page 350 & 0101-8175 & 0 & \\
\hline Revista Brasileira de Zoologia & v. 21, fisrt page 351, last page 356 & 0101-8175 & 0 & \\
\hline Revista Brasileira de Zoologia & v. 21 , fisrt page 357, last page 369 & $0101-8175$ & 0 & \\
\hline Revista Brasileira de Zoologia & v. 21, fisrt page 371, last page 377 & 0101-8175 & 0 & \\
\hline Revista Brasileira de Zoologia & v. 21, fisrt page 391 , last page 395 & 0101-8175 & 0 & \\
\hline Revista Brasileira de Zoologia & v. 21, fisrt page 397, last page 402 & 0101-8175 & 0 & \\
\hline Revista Brasileira de Zoologia & v. 21 , fisrt page 403 , last page 404 & $0101-8175$ & 0 & \\
\hline Revista Brasileira de Zoologia & v. 21, fisrt page 405, last page 407 & 0101-8175 & 0 & \\
\hline Revista Brasileira de Zoologia & v. 21 , fisrt page 410 , last page 415 & $0101-8175$ & 0 & \\
\hline Revista Brasileira de Zoologia & v. 21 , fisrt page 421 , last page 433 & 0101-8175 & 0 & \\
\hline Revista Brasileira de Zoologia & v. 21 , fisrt page 435 , last page 437 & $0101-8175$ & 0 & \\
\hline Revista Brasileira de Zoologia & v. 21, fisrt page 439, last page 448 & 0101-8175 & 0 & \\
\hline Revista Brasileira de Zoologia & v. 21 , fisrt page 449 , last page 452 & $0101-8175$ & 0 & \\
\hline Revista Brasileira de Zoologia & v. 21 , fisrt page 45 , last page 50 & 0101-8175 & 0 & \\
\hline Revista Brasileira de Zoologia & v. 21 , fisrt page 453 , last page 458 & $0101-8175$ & 0 & \\
\hline Revista Brasileira de Zoologia & v. 21 , fisrt page 467 , last page 475 & 0101-8175 & 0 & \\
\hline Revista Brasileira de Zoologia & v. 21 , fisrt page 477 , last page 486 & $0101-8175$ & 0 & \\
\hline Revista Brasileira de Zoologia & v. 21 , fisrt page 487 , last page 489 & 0101-8175 & 0 & \\
\hline Revista Brasileira de Zoologia & v. 21, fisrt page 491, last page 498 & $0101-8175$ & 0 & \\
\hline
\end{tabular}


Journal

Revista Brasileira de Zoologia

Revista Brasileira de Zoologia

Revista Brasileira de Zoologia

Revista Brasileira de Zoologia

Revista Brasileira de Zoologia

Revista Brasileira de Zoologia

Revista Brasileira de Zoologia

Revista Brasileira de Zoologia

Revista Brasileira de Zoologia

Revista Brasileira de Zoologia

Revista Brasileira de Zoologia

Revista Brasileira de Zoologia

Revista Brasileira de Zoologia

Revista Brasileira de Zoologia

Revista Brasileira de Zoologia

Revista Brasileira de Zoologia

Revista Brasileira de Zoologia

Revista Brasileira de Zoologia

Revista Brasileira de Zoologia

Revista Brasileira de Zoologia

Revista Brasileira de Zoologia

Revista Brasileira de Zoologia

Revista Brasileira de Zoologia

Revista Brasileira de Zoologia

Revista Brasileira de Zoologia

Revista Brasileira de Zoologia

Revista Brasileira de Zoologia

Revista Brasileira de Zoologia

Revista Brasileira de Zoologia

Revista Brasileira de Zoologia

Revista Brasileira de Zoologia

Revista Brasileira de Zoologia

Revista Brasileira de Zoologia

Revista Brasileira de Zoologia

Revista Brasileira de Zoologia

Revista Brasileira de Zoologia

Revista Brasileira de Zoologia

Revista Brasileira de Zoologia

Revista Brasileira de Zoologia

Revista Brasileira de Zoologia

Revista Brasileira de Zoologia

Revista Brasileira de Zoologia

Revista Brasileira de Zoologia

Revista Brasileira de Zoologia

Revista Brasileira de Zoologia

Revista Brasileira de Zoologia

Revista Brasileira de Zoologia

Revista Brasileira de Zoologia

Revista Brasileira de Zoologia

Revista Brasileira de Zoologia

Revista Brasileira de Zoologia

Revista Brasileira de Zoologia

Revista Brasileira de Zoologia

Revista Brasileira de Zoologia

Revista Brasileira de Zoologia

Revista Brasileira de Zoologia

Revista Brasileira de Zoologia

Revista Brasileira de Zoologia

Revista Brasileira de Zoologia

Revista Brasileira de Zoologia
Paper info.

ISSN

0101-8175

$0101-8175$

0101-8175

$0101-8175$

0101-8175

$0101-8175$

0101-8175

0101-8175

0101-8175

0101-8175

0101-8175

$0101-8175$

0101-8175

0101-8175

0101-8175

0101-8175

$0101-8175$

0101-8175

0101-8175

0101-8175

0101-8175

$0101-8175$

$0101-8175$

0101-8175

0101-8175

0101-8175

0101-8175

0101-8175

0101-8175

0101-8175

$0101-8175$

0101-8175

0101-8175

0101-8175

0101-8175

0101-8175

0101-8175

0101-8175

0101-8175

0101-8175

0101-8175

0101-8175

0101-8175

0101-8175

0101-8175

0101-8175

0101-8175

0101-8175

0101-8175

0101-8175

0101-8175

0101-8175

0101-8175

0101-8175

0101-8175

0101-8175

0101-8175

0101-8175

0101-8175

0101-8175
Half-Life

0

0

0

0

0

0

0

0

0

0

0

0

0

0

0

0

0

0

0

0

0

0

0

0

0

0

0

0

0

0

0

0

0

0

0

0

0

0

0

0

0

0

0

0

0

0

0

0

0

0

0

0

0

0

0

0

0 
Journal

Revista Brasileira de Zoologia

Revista Brasileira de Zoologia

Revista Brasileira de Zoologia

Revista Brasileira de Zoologia

Revista Brasileira de Zoologia

Revista Brasileira de Zoologia

Revista Brasileira de Zoologia

Revista Brasileira de Zoologia

Revista Brasileira de Zoologia

Revista Brasileira de Zoologia

Revista Brasileira de Zoologia

Revista Brasileira de Zoologia

Revista Brasileira de Zoologia

Revista Brasileira de Zoologia

Revista Brasileira de Zoologia

Revista Brasileira de Zoologia

Revista Brasileira de Zoologia

Revista Brasileira de Zoologia

Revista Brasileira de Zoologia

Revista Brasileira de Zoologia

Revista Brasileira de Zoologia

Revista Brasileira de Zoologia

Revista Brasileira de Zoologia

Revista Brasileira de Zoologia

Revista Brasileira de Zoologia

Revista Brasileira de Zoologia

Revista Brasileira de Zoologia

Revista Brasileira de Zoologia

Revista Brasileira de Zoologia

Revista Brasileira de Zoologia

Revista Brasileira de Zoologia

Revista Brasileira de Zoologia

Revista Brasileira de Zoologia

Revista Brasileira de Zoologia

Revista Brasileira de Zoologia

Revista Brasileira de Zoologia

Revista Brasileira de Zoologia

Revista Brasileira de Zoologia

Revista Brasileira de Zoologia

Revista Brasileira de Zoologia

Revista Brasileira de Zoologia

Revista Brasileira de Zoologia

Revista Brasileira de Zoologia

Revista Brasileira de Zoologia

Revista Brasileira de Zoologia

Revista Brasileira de Zoologia

Revista Brasileira de Zoologia

Revista Brasileira de Zoologia

Revista Brasileira de Zoologia

Revista Brasileira de Zoologia

Revista Brasileira de Zoologia

Revista Brasileira de Zoologia

Revista Brasileira de Zoologia

Revista Brasileira de Zoologia

Revista Brasileira de Zoologia

Revista Brasileira de Zoologia

Revista Brasileira de Zoologia

Revista Brasileira de Zoologia

Revista Brasileira de Zoologia

Revista Brasileira de Zoologia
Paper info.

v. 22, fistt page 197, last page 204

v. 22, fisrt page 205, last page 211

v. 22 , fisrt page 213 , last page 217

v. 22 , fisrt page 219 , last page 223

v. 22, fisrt page 225, last page 229

v. 22 , fist page 23 , last page 34

v. 22 , fisrt page 285 , last page 291

v. 22, fisrt page 293, last page 305

v. 22, fisrt page 306 , last page 312

v. 22 , fisrt page 320 , last page 325

v. 22 , fisrt page 339 , last page 341

v. 22 , fisrt page 349 , last page 353

v. 22 , fisrt page 35 , last page 42

v. 22 , fisrt page 366 , last page 371

v. 22 , fisrt page 372 , last page 382

v. 22 , fisrt page 410 , last page 418

v. 22 , fisrt page 43 , last page 50

v. 22 , fisrt page 454 , last page 460

v. 22 , fisrt page 461 , last page 476

v. 22 , fisrt page 477 , last page 483

v. 22 , fisrt page 494 , last page 500

v. 22 , fisrt page 51 , last page 59

v. 22 , fisrt page 511 , last page 513

v. 22, fisrt page 517 , last page 521

v. 22 , fisrt page 529 , last page 551

v. 22, fisrt page 552, last page 555

v. 22 , fisrt page 556 , last page 564

v. 22 , fisrt page 565 , last page 570

v. 22, fisrt page 571 , last page 572

v. 22 , fisrt page 573 , last page 579

v. 22 , fisrt page 580 , last page 586

v. 22, fisrt page 591, last page 595

v. 22 , fisrt page 61 , last page 72

v. 22, fisrt page 619 , last page 622

v. 22 , fisrt page 623 , last page 632

v. 22 , fisrt page 645 , last page 647

v. 22 , fisrt page 656 , last page 661

v. 22 , fisrt page 662 , last page 666

v. 22 , fisrt page 667 , last page 675

v. 22 , fisrt page 676 , last page 679

v. 22 , fisrt page 680 , last page 686

v. 22 , fisrt page 687 , last page 695

v. 22 , fisrt page 696 , last page 698

v. 22 , fisrt page 709 , last page 711

v. 22 , fisrt page 717 , last page 723

v. 22 , fisrt page 724 , last page 734

v. 22 , fisrt page 73 , last page 80

v. 22 , fisrt page 753 , last page 760

v. 22 , fisrt page 761 , last page 763

v. 22 , fisrt page 771 , last page 774

v. 22 , fisrt page 780 , last page 783

v. 22 , fisrt page 793 , last page 795

v. 22 , fisrt page 796 , last page 805

v. 22 , fisrt page 801 , last page 811

v. 22 , fist page 81 , last page 83

v. 22 , fisrt page 812 , last page 815

v. 22 , fisrt page 816 , last page 818

v. 22 , fisrt page 819 , last page 821

v. 22 , fisrt page 821 , last page 824

v. 22 , fisrt page 830 , last page 832
ISSN

0101-8175

IF

0101-8175

0101-8175

0101-8175

0101-8175

0101-8175

0101-8175

0101-8175

0101-8175

0101-8175

0101-8175

0101-8175

0101-8175

0101-8175

0101-8175

0101-8175

0101-8175

0101-8175

0101-8175

0101-8175

0101-8175

0101-8175

0101-8175

0101-8175

0101-8175

0101-8175

0101-8175

0101-8175

0101-8175

0101-8175

0101-8175

0101-8175

0101-8175

0101-8175

0101-8175

0101-8175

0101-8175

0101-8175

0101-8175

0101-8175

0101-8175

0101-8175

0101-8175

0101-8175

0101-8175

0101-8175

0101-8175

0101-8175

0101-8175

0101-8175

0101-8175

0101-8175

0101-8175

0101-8175

0101-8175

0101-8175

0101-8175

0101-8175

0101-8175

0101-8175
0

0

0

0

0

\section{0}

\section{0}

\section{0}

\section{0}

0

\section{0}

0

\section{0}

0

0

0

0

0

0

0

0

0

0

0

\section{0}

\section{0}

\section{0}

0 
Journal

Revista Brasileira de Zoologia

Revista Brasileira de Zoologia

Revista Brasileira de Zoologia

Revista Brasileira de Zoologia

Revista Brasileira de Zoologia

Revista Brasileira de Zoologia

Revista Brasileira de Zoologia

Revista Brasileira de Zoologia

Revista Brasileira de Zoologia

Revista Brasileira de Zoologia

Revista Brasileira de Zoologia

Revista Brasileira de Zoologia

Revista Brasileira de Zoologia

Revista Brasileira de Zoologia

Revista Brasileira de Zoologia

Revista Brasileira de Zoologia

Revista Brasileira de Zoologia

Revista Brasileira de Zoologia

Revista Brasileira de Zoologia

Revista Brasileira de Zoologia

Revista Brasileira de Zoologia

Revista Brasileira de Zoologia

Revista Brasileira de Zoologia

Revista Brasileira de Zoologia

Revista Brasileira de Zoologia

Revista Brasileira de Zoologia

Revista Brasileira de Zoologia

Revista Brasileira de Zoologia

Revista Brasileira de Zoologia

Revista Brasileira de Zoologia

Revista Brasileira de Zoologia

Revista Brasileira de Zoologia

Revista Brasileira de Zoologia

Revista Brasileira de Zoologia

Revista Brasileira de Zoologia

Revista Brasileira de Zoologia

Revista Brasileira de Zoologia

Revista Brasileira de Zoologia

Revista Brasileira de Zoologia

Revista Brasileira de Zoologia

Revista Brasileira de Zoologia

Revista Brasileira de Zoologia

Revista Brasileira de Zoologia

Revista Brasileira de Zoologia

Revista Brasileira de Zoologia

Revista Brasileira de Zoologia

Revista Brasileira de Zoologia

Revista Brasileira de Zoologia

Revista Brasileira de Zoologia

Revista Brasileira de Zoologia

Revista Brasileira de Zoologia

Revista Brasileira de Zoologia

Revista Brasileira de Zoologia

Revista Brasileira de Zoologia

Revista Brasileira de Zoologia

Revista Brasileira de Zoologia

Revista Brasileira de Zoologia

Revista Brasileira de Zoologia

Revista Brasileira de Zoologia

Revista Brasileira de Zoologia
Paper info.

ISSN

0101-8175

0101-8175

0101-8175

$0101-8175$

0101-8175

$0101-8175$

0101-8175

0101-8175

0101-8175

0101-8175

0101-8175

0101-8175

0101-8175

0101-8175

0101-8175

0101-8175

$0101-8175$

0101-8175

0101-8175

0101-8175

0101-8175

$0101-8175$

0101-8175

0101-8175

0101-8175

0101-8175

0101-8175

0101-8175

0101-8175

0101-8175

0101-8175

0101-8175

0101-8175

0101-8175

0101-8175

0101-8175

0101-8175

0101-8175

0101-8175

0101-8175

0101-8175

0101-8175

0101-8175

0101-8175

0101-8175

0101-8175

0101-8175

0101-8175

0101-8175

0101-8175

0101-8175

0101-8175

0101-8175

0101-8175

0101-8175

0101-8175

0101-8175

0101-8175

0101-8175

0101-8175
Half-Life

0

0

0

0

0

0

0

0

0

0

0

0

0

0

0

0

0

0

0

0

0

0

0

0

0

0

0

0

0

0

0

0

0

0

0

0

0

0

0

0

0

0

0

0

0

0

0

0

0

0

0

0

0

0

0

0

0

0 


\begin{tabular}{|c|c|c|c|c|}
\hline $\begin{array}{c}\text { Journal } \\
\end{array}$ & Paper info. & ISSN & IF & Half-Life \\
\hline Revista Brasileira de Zoologia & v. 23, fisrt page 298 , last page 299 & $0101-8175$ & 0 & \\
\hline Revista Brasileira de Zoologia & v. 23 , fisrt page 307 , last page 310 & 0101-8175 & 0 & \\
\hline Revista Brasileira de Zoologia & v. 23, fisrt page 314 , last page 322 & 0101-8175 & 0 & \\
\hline Revista Brasileira de Zoologia & v. 23 , fisrt page 323 , last page 330 & 0101-8175 & 0 & \\
\hline Revista Brasileira de Zoologia & v. 23 , fistt page 331 , last page 339 & 0101-8175 & 0 & \\
\hline Revista Brasileira de Zoologia & v. 23 , fistt page 357 , last page 363 & $0101-8175$ & 0 & \\
\hline Revista Brasileira de Zoologia & v. 23, fisrt page 364 , last page 366 & $0101-8175$ & 0 & \\
\hline Revista Brasileira de Zoologia & v. 23 , fisrt page 367 , last page 374 & 0101-8175 & 0 & \\
\hline Revista Brasileira de Zoologia & v. 23 , fistt page 375 , last page 380 & $0101-8175$ & 0 & \\
\hline Revista Brasileira de Zoologia & v. 23 , fistt page 395 , last page 403 & $0101-8175$ & 0 & \\
\hline Revista Brasileira de Zoologia & v. 23 , fistt page 408 , last page 413 & $0101-8175$ & 0 & \\
\hline Revista Brasileira de Zoologia & v. 23 , fisrt page 414 , last page 420 & 0101-8175 & 0 & \\
\hline Revista Brasileira de Zoologia & v. 23, fisrt page 42 , last page 49 & $0101-8175$ & 0 & \\
\hline Revista Brasileira de Zoologia & v. 23 , fisrt page 421 , last page 428 & $0101-8175$ & 0 & \\
\hline Revista Brasileira de Zoologia & v. 23 , fisrt page 429 , last page 442 & 0101-8175 & 0 & \\
\hline Revista Brasileira de Zoologia & v. 23, fisrt page 443 , last page 454 & 0101-8175 & 0 & \\
\hline Revista Brasileira de Zoologia & v. 23 , fisrt page 455 , last page 459 & 0101-8175 & 0 & \\
\hline Revista Brasileira de Zoologia & v. 23 , fisrt page 480 , last page 489 & 0101-8175 & 0 & \\
\hline Revista Brasileira de Zoologia & v. 23, fisrt page 490 , last page 496 & $0101-8175$ & 0 & \\
\hline Revista Brasileira de Zoologia & v. 23 , fisrt page 497 , last page 508 & $0101-8175$ & 0 & \\
\hline Revista Brasileira de Zoologia & v. 23 , fisrt page 520 , last page 528 & $0101-8175$ & 0 & \\
\hline Revista Brasileira de Zoologia & v. 23 , fisrt page 529 , last page 536 & $0101-8175$ & 0 & \\
\hline Revista Brasileira de Zoologia & v. 23 , fisrt page 537 , last page 546 & $0101-8175$ & 0 & \\
\hline Revista Brasileira de Zoologia & v. 23 , fisrt page 547 , last page 549 & $0101-8175$ & 0 & \\
\hline Revista Brasileira de Zoologia & v. 23, fisrt page 550 , last page 568 & 0101-8175 & 0 & \\
\hline Revista Brasileira de Zoologia & v. 23, fisrt page 584 , last page 587 & $1806-969 x$ & 0 & \\
\hline Revista Brasileira de Zoologia & v. 23, fisrt page 608 , last page 629 & 0101-8175 & 0 & \\
\hline Revista Brasileira de Zoologia & v. 23 , fisrt page 642 , last page 651 & $0101-8175$ & 0 & \\
\hline Revista Brasileira de Zoologia & v. 23 , fisrt page 678 , last page 691 & $0101-8175$ & 0 & \\
\hline Revista Brasileira de Zoologia & v. 23, fisrt page 703 , last page 706 & $0101-8175$ & 0 & \\
\hline Revista Brasileira de Zoologia & v. 23 , fisrt page 707 , last page 710 & $0101-8175$ & 0 & \\
\hline Revista Brasileira de Zoologia & v. 23, fisrt page 711 , last page 715 & $0101-8175$ & 0 & \\
\hline Revista Brasileira de Zoologia & v. 23 , fisrt page 733 , last page 739 & $0101-8175$ & 0 & \\
\hline Revista Brasileira de Zoologia & v. 23 , fisrt page 746 , last page 757 & $0101-8175$ & 0 & \\
\hline Revista Brasileira de Zoologia & v. 23 , fisrt page 758 , last page 763 & $0101-8175$ & 0 & \\
\hline Revista Brasileira de Zoologia & v. 23 , fisrt page 764 , last page 778 & 0101-8175 & 0 & \\
\hline Revista Brasileira de Zoologia & v. 23 , fisrt page 779 , last page 788 & $0101-8175$ & 0 & \\
\hline Revista Brasileira de Zoologia & v. 23, fisrt page 796 , last page 806 & $0101-8175$ & 0 & \\
\hline Revista Brasileira de Zoologia & v. 23 , fisrt page 806 , last page 809 & $0101-8175$ & 0 & \\
\hline Revista Brasileira de Zoologia & v. 23 , fisrt page 807 , last page 812 & $0101-8175$ & 0 & \\
\hline Revista Brasileira de Zoologia & v. 23 , fisrt page 813 , last page 816 & $0101-8175$ & 0 & \\
\hline Revista Brasileira de Zoologia & v. 23 , fisrt page 824 , last page 831 & $0101-8175$ & 0 & \\
\hline Revista Brasileira de Zoologia & v. 23 , fisrt page 832 , last page 840 & $0101-8175$ & 0 & \\
\hline Revista Brasileira de Zoologia & v. 23 , fisrt page 841 , last page 864 & 0101-8175 & 0 & \\
\hline Revista Brasileira de Zoologia & v. 23 , fisrt page 865 , last page 869 & 0101-8175 & 0 & \\
\hline Revista Brasileira de Zoologia & v. 23 , fisrt page 877 , last page 879 & 0101-8175 & 0 & \\
\hline Revista Brasileira de Zoologia & v. 23 , fisrt page 879 , last page 882 & $0101-8175$ & 0 & \\
\hline Revista Brasileira de Zoologia & v. 23 , fisrt page 886 , last page 887 & 0101-8175 & 0 & \\
\hline Revista Brasileira de Zoologia & v. 23 , fisrt page 888 , last page 890 & 0101-8175 & 0 & \\
\hline Revista Brasileira de Zoologia & v. 23, fisrt page 891 , last page 893 & $0101-8175$ & 0 & \\
\hline Revista Brasileira de Zoologia & v. 23, fisrt page 901 , last page 914 & 0101-8175 & 0 & \\
\hline Revista Brasileira de Zoologia & v. 24 , fisrt page 847 , last page 855 & 0101-8175 & 0 & \\
\hline Revista Brasileira de Zoologia & v. 32 , fisrt page 350 , last page 356 & 0101-8175 & 0 & \\
\hline Revista Brasileira de Zoologia & v. 32 , fisrt page 460 , last page 470 & 0101-8175 & 0 & \\
\hline Revista Brasileira de Zoologia & v. 35 , fisrt page 483 , last page 488 & 0101-8175 & 0 & \\
\hline Revista Brasileira de Zoologia & v. 6 , fisrt page 115 , last page 127 & 0101-8175 & 0 & \\
\hline Revista Brasileira de Zoologia & v. n. 22 , fistt page 613 , last page 618 & 0101-8175 & 0 & \\
\hline Median & & & 0.612 & \\
\hline
\end{tabular}

